University of Louisville

ThinkIR: The University of Louisville's Institutional Repository

Electronic Theses and Dissertations

$12-2010$

\title{
Topology and function of nyctalopin in yeast and in-vitro translation systems.
}

Pasano Pasano Bojang

University of Louisville

Follow this and additional works at: https://ir.library.louisville.edu/etd

\section{Recommended Citation}

Bojang, Pasano Pasano, "Topology and function of nyctalopin in yeast and in-vitro translation systems." (2010). Electronic Theses and Dissertations. Paper 124.

https://doi.org/10.18297/etd/124

This Doctoral Dissertation is brought to you for free and open access by ThinkIR: The University of Louisville's Institutional Repository. It has been accepted for inclusion in Electronic Theses and Dissertations by an authorized administrator of ThinkIR: The University of Louisville's Institutional Repository. This title appears here courtesy of the author, who has retained all other copyrights. For more information, please contact thinkir@louisville.edu. 


\title{
TOPOLOGY AND FUNCTION OF NYCTALOPIN IN YEAST AND IN VITRO TRANSLATION SYSTEMS
}

\author{
By \\ Pasano Bojang Jr. \\ B.Sc., Kentucky State University, 2004 \\ Frankfort, Kentucky
}

A Dissertation submitted to the faculty of the Graduate School of the University of Louisville in Partial fulfillment of the requirement for Degree of

\author{
Doctor of Philosophy
}
Department of Biochemistry and Molecular Biology University of Louisville
Louisville, Kentucky

December, 2010 

TOPOLOGY AND FUNCTION OF NYCTALOPIN IN YEAST AND IN VITRO

TRANSLATION SYSTEMS

By

Pasano Bojang Jr.

B.Sc., Kentucky State University, 2004

Frankfort, Kentucky

A Dissertation Approved on

July 292010

By the following Dissertation Corpmittee:

Ronald G Gregg, PhD - Chair

Steven Ellis, PhD

\begin{tabular}{c}
\hline Richărd C Feldhoff, PhD \\
Maureen A McCalí, PhD
\end{tabular}

Chuan $\mathrm{Hu}, \mathrm{PhD}$ 


\section{DEDICATION}

To my family

Aja-Penku Bojang (Mother), Isatou Jatta-Bojang (Wife), and Alaji-Lamin Bojang (Son) 


\section{ACKNOWLEDGMENTS}

I would like to sincerely thank my mentor, Dr. Ronald G. Gregg for his scientific guidance and support. I would also like to thank all of my committee members, Dr. Maureen McCall, Dr. Steven Ellis, Dr. Chuan Hu, and Dr. Richard Feldhoff for their scientific insight and help throughout the course of my project. Special thanks to my parents especially my mother who did everything to make sure I got an excellent education. I would like to give many thanks to my wife (Isatou Jatta-Bojang) and son (Alaji-Lamin Bojang) for their unconditional love, support, patience and encouragement. I am very grateful to all the former and present members of the Gregg and McCall labs for their support. I would also like to thank the entire Ramos lab especially Dr. Vilius Stribinkis for helping me with the yeast work. I would like to thank my entire extended family for their unyielding support. I am very thankful to my brother Alhagie E.F Conteh for always being there for me whenever I need him. I am also very grateful to the faculty and staff of the department of biochemistry and molecular biology for their continuous support. Finally I would like to give thanks to GOD for this unique opportunity. 


\begin{abstract}
TOPOLOGY AND FUNCTION OF NYCTALOPIN IN YEAST AND IN VITRO

TRANSLATION SYSTEMS

Pasano Bojang Jr.
\end{abstract}

July 29,2010

Congenital stationary night blindness 1 (CSNB1) is a genetic disorder characterized in humans by night blindness, low visual acuity and myopia. CSNB1 is caused by defects in genes that are involved in signaling between photoreceptors and depolarizing bipolar cells (DBCs). DBCs utilize a metabotropic glutamate receptor-6 (Grm6) cascade that modulates the activity of a non-specific cation channel. CSNB1 is diagnosed by a reduced $b$-wave in the electroretinogram (ERG). A b-wave indicates that DBCs are depolarized in response to a flash of light. In the dark, there is a tonic release of glutamate from the photoreceptors into the synaptic cleft. This glutamate binds to the Grm6 receptor, activating a G-protein signal transduction cascade that closes a nonselective cation channel. The Gregg laboratory has identified this channel as the transient receptor potential melastatin $1(\mathrm{Trpm} 1)$ channel. The focus of my research is to determine how another protein, nyctalopin, which also lacks b-wave causes a loss of the channel's activity. Nyctalopin is an integral membrane protein with the entire leucine rich repeat in the extracellular space. Nyctalopin interacts directly with extracellular loops of Trpml. Although nyctalopin alone is not able to gate or traffic the Trpml channel to the 
membrane, gene expression profiling and membrane split ubiquitin yeast two hybrid screen suggest that a complex of proteins including nyctalopin are involved in either assembling or trafficking of the Trpml channel to the plasma membrane. 
TABLE OF CONTENTS

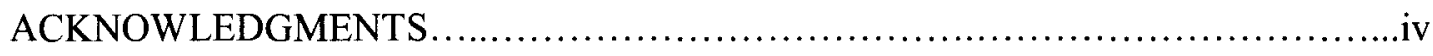

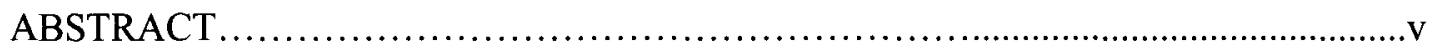

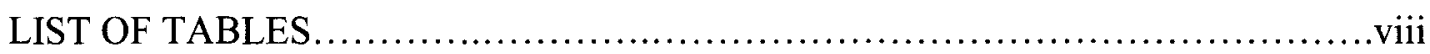

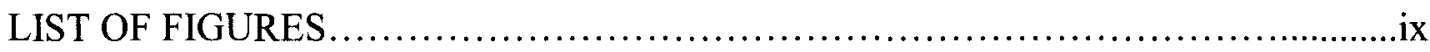

CHAPTERS

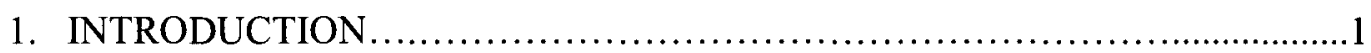

2. THE TOPOLOGY AND HOMODIMERIZATION OF NYCTALOPIN

3. INTERACTION PARTNERS OF NYCTALOPIN AND ROLE

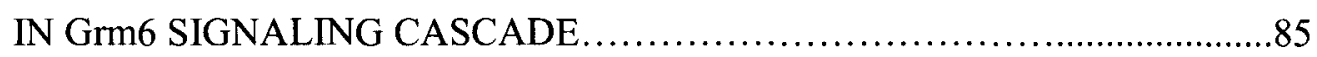

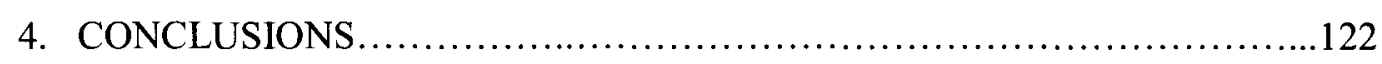

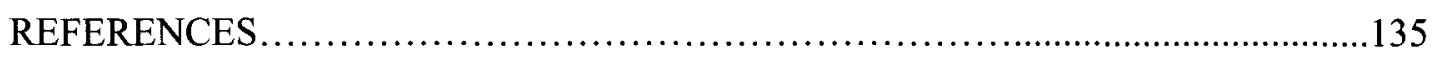

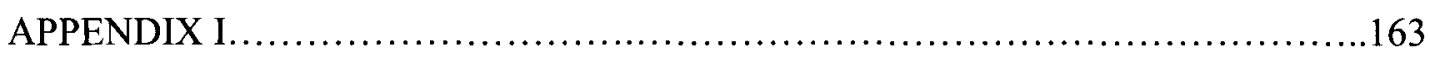

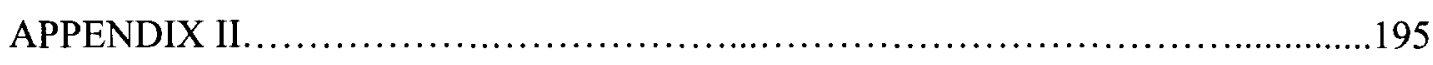

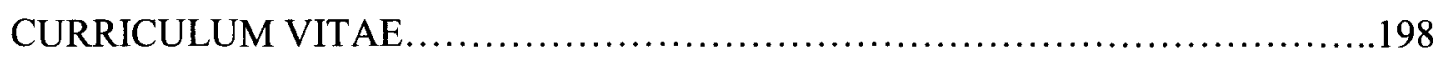




\section{LIST OF TABLES}

TABLE PAGE

1. Predicted membrane topology of nyctalopin.......................55

2. Summary of the complexity of the retinal cDNA library $\ldots \ldots \ldots \ldots \ldots \ldots 119$

3. List of potential interacting partners for nyctalopin ...................120

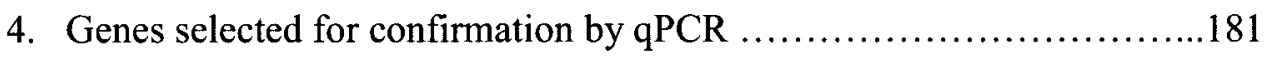




\section{LIST OF FIGURES}

$\begin{array}{lll}\text { FIGURE PAGE } & \text { PAT }\end{array}$

1. Transverse section of the human eye and the retina.................................

2. A cross section of an immunostained mouse retina...........................6

3. Summary of the rod and cone pathways in the retina......................... 8

4. The structure of a ribbon synapse....................................... 11

5. Dark and light adapted ERG of normal mouse retina............................21

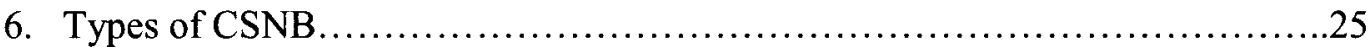

7. Representative scotopic ERG from three adult males............................27

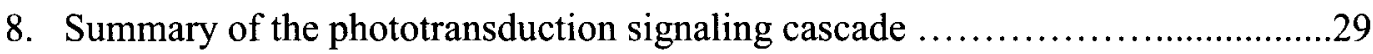

9. Summary of the signal transduction cascade in the DBC.............................36

10. Tertiary structure of nyctalopin in a horseshoe

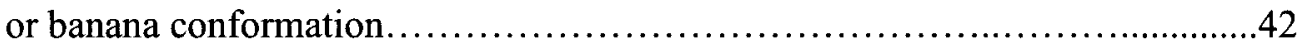

11. The SRP-mediated co-translational

targeting of protein through the ER ..................................46

12. Schematic drawing of the different

predicted membrane topologies of nyctalopin............................57

13. Schematic representation of membrane split ubiquitin

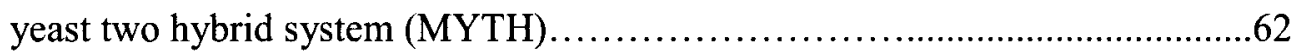

14. Nyctalopin is localized to the membrane in yeast ................................65 
15. The N-terminus of nyctalopin is extracellular

16. Schematic of the different predicted membrane topologies of nyctalopin

17. Genetic analysis shows murine nyctalopin has a single transmembrane domain .73

18. Nyctalopin does not form dimers in yeast................................76

19. The $\mathrm{N}$-terminus of nyctalopin is in the lumen of the ER .....................78

20. The N-terminus of nyctalopin is in the lumen of the ER .........................80

21. Methodology used to generate retina cDNA for the library...........................99

22. Retinal cDNA library Construction ..................................................101

23. Membrane split ubiquitin yeast two hybrid (MYTH) analysis..................103

24. Nyctalopin interacts with Trpm1 in the MYTH system ...........................106

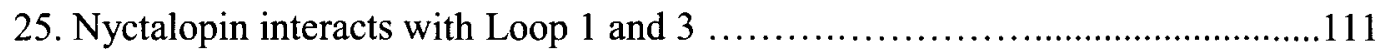

26. Functional analysis of Trpm1 in yeast cells.........................................114

27. Trp channel gating through Calcium Influx factor (CIF)...................... 128

28. Trpm1 gating through Diacylglycerol (DAG) ...........................131

29. Trafficking as a way of gating Trpml channel

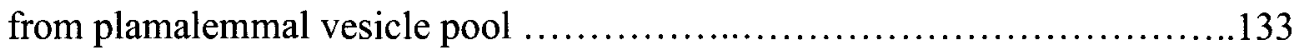

30. Developmental expression of genes expressed in the OPL..............173-177

31. Scatter plot showing variation in gene expression between

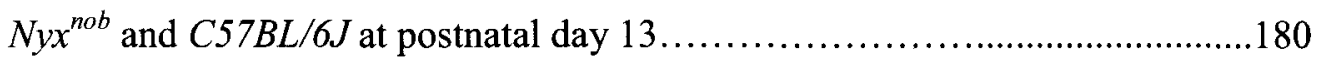

32. Genes with altered expression levels in $N y x^{n o b}$ mice........................ 184

33. RT-PCR analysis of genes that showed significantly 
different expression level between $N y x^{\text {nob }}$ and

$C 57 B L / 6 J$ as determined by t-test.................................... 188

34. Temporal expression pattern of genes differentially

regulated in $\mathrm{Nyx^{nob }}$ mice.......................................... 190 


\section{INTRODUCTION}

\section{Overview of Vision}

Our eyes provide us with sensory input about our environment. Although most eye diseases are not lethal, they can impair our ability to interact with our surroundings in a normal way. According to the National Eye Institute (NEI), 314 million people are visually impaired world wide. Of these, 45 million are completely blind and about twothird are females. The Department of Health and Human Services estimates that 80 million Americans suffer from potentially blinding eye diseases and 6 million new cases occur each year (PBA-Senate FY 2010 LHHS Written Testimony).

Vision is initiated when light enters the eye, passes through the cornea, the lens, the vitreous and is focused on the retina (Figure 1). The retina is the light sensitive part of the eye. It consists of specialized neurons that are able to convert light energy into an electrical signal and relay it to the brain for visual processing. The systematic transfer of signal from neuron to neuron in the retina is accomplished by signaling through a highly organized structure that was originally described by Santiago Ramon y Cajal (Cajal et al., 1894).

The neutral retina is divided into three cellular layers: the photoreceptor layer, inner nuclear layer (INL) and the ganglion cell layer (Figure 2). Synapses between the bipolar cells, horizontal cells and photoreceptors form the outer plexiform layer (OPL), and synapses between ganglion cells, amacrine cells and bipolar cells forms the inner plexiform (IPL) layer. The photoreceptors consist of two types: the rods and the cones. 
Figure 1. Transverse section of the human eye and the retina. (Left) A drawing of the cross-section of the human eye showing the retina (red) at the back of the eye. The green arrow depicts a light ray as it moves through the cornea, the lens, the vitreous and focused on the retina. (Right) A schematic of a cross-section of the retina showing the lamination of the different types of neuronal cells. The green arrow depicts the flow of light energy to the ROS of photoreceptors where it activates rhodopsin, which converts the light energy into a chemical signal. The red arrow depicts the flow of the chemical signal from the photoreceptors to the ganglion cells. (Adapted from webvision http:webvision.med.utah.edu) 
EYE

RETINA

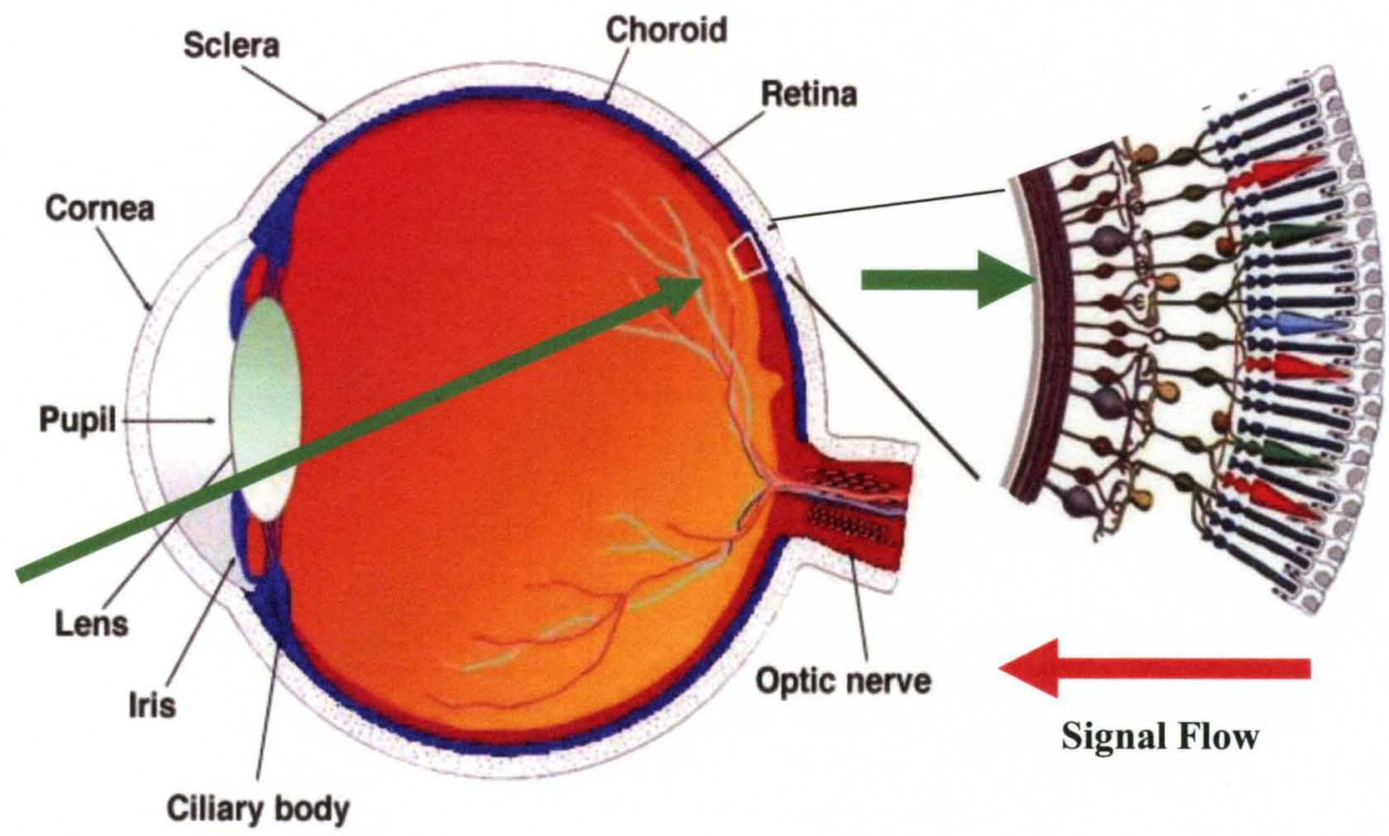


Rods detect light under scotopic conditions while cones detect light under photopic conditions. There is a single type of rod while there are up to three types of cones. Functionally there are two types of bipolar cells, those that depolarize (DBC or $\mathrm{ON}$ ) in response to a light increment, and those that hyperpolarize (HBC or OFF) in response to a light increment (Kolb and Nelson et al., 1995; Saito and Kaneko, 1983; Attwell et al., 1987; Nawy \& Jahr, 1991; Masu et al., 1995). Both depolarizing bipolar cells (DBCs) and hyperpolarizing bipolar cell (HBCs) form synapses with rods and cones (Brandstatter and Hack, 2001). Morphologically, there is a single type of rod bipolar cell and up to ten different types of cone bipolar cells in the retina (Mariani et al., 1981; Boycott and Wässle, 1991; Euler and Wässle, 1995; Ghosh et al., 2004; MacNeil et al., 2004; Pignatelli and Strettoi, 2004). The cone bipolar cells form synapses with the amacrine cells and ganglion cells. Rod bipolar cells do not synapse directly on to ganglion cells rather they form synapses with AII amacrine cells that synapse on to depolarizing and hyperpolarizing cone bipolar cells (DeVries and Baylor, 1995; Saucy et al., 1998). There are 30 different types of amacrine cells involved in the lateral connections between the bipolar cells and ganglion cells. The ganglion cell axons form the optic nerve that transmits the signal to the brain (Figure 2).

Signal transduction in the retina starts at the photoreceptor outer segments. Light energy is converted into chemical signal by the phototransduction cascade; which leads to the closure of cyclic GMP (cGMP) channels (CNG) on the photoreceptor outer segments. The closure of these channels leads to hyperpolarization, which in turn leads to the closure of voltage dependent calcium channels (VDCC) at the photoreceptor terminals. The closure of VDCC results in a decrease in the release of glutamate into the synaptic cleft, 
Figure 2. A cross section of an immunostained mouse retina (Left) Cross-section of adult mouse retina immunostained for cone arrestin (bluish purple) to show photoreceptors (PR), calbindin (red) to show one class of amacrine cells (ACs) and ganglion cells (GCs) and GFP (green) to show depolarizing bipolar cells. GFP stained depolarizing bipolar cell are from a transgenic mouse that expresses GFP under the control of Grm6 promoter (Dhingra et al., 2008). (Right) A schematic drawing showing the lamination and synaptic connections of photoreceptors, to horizontal cells (HC) and bipolar cells (BCs) in the OPL, rod bipolar cells to AII amacrine cell in the ON sublamina of IPL and cone depolarizing bipolar and hyperpolarizing bipolar cells to amacrine cell and ganglion cell in both the ON (IPL-B) and OFF (IPL-A) sublaminae of the IPL, respectively. (Morgan and Wong, 2005). 

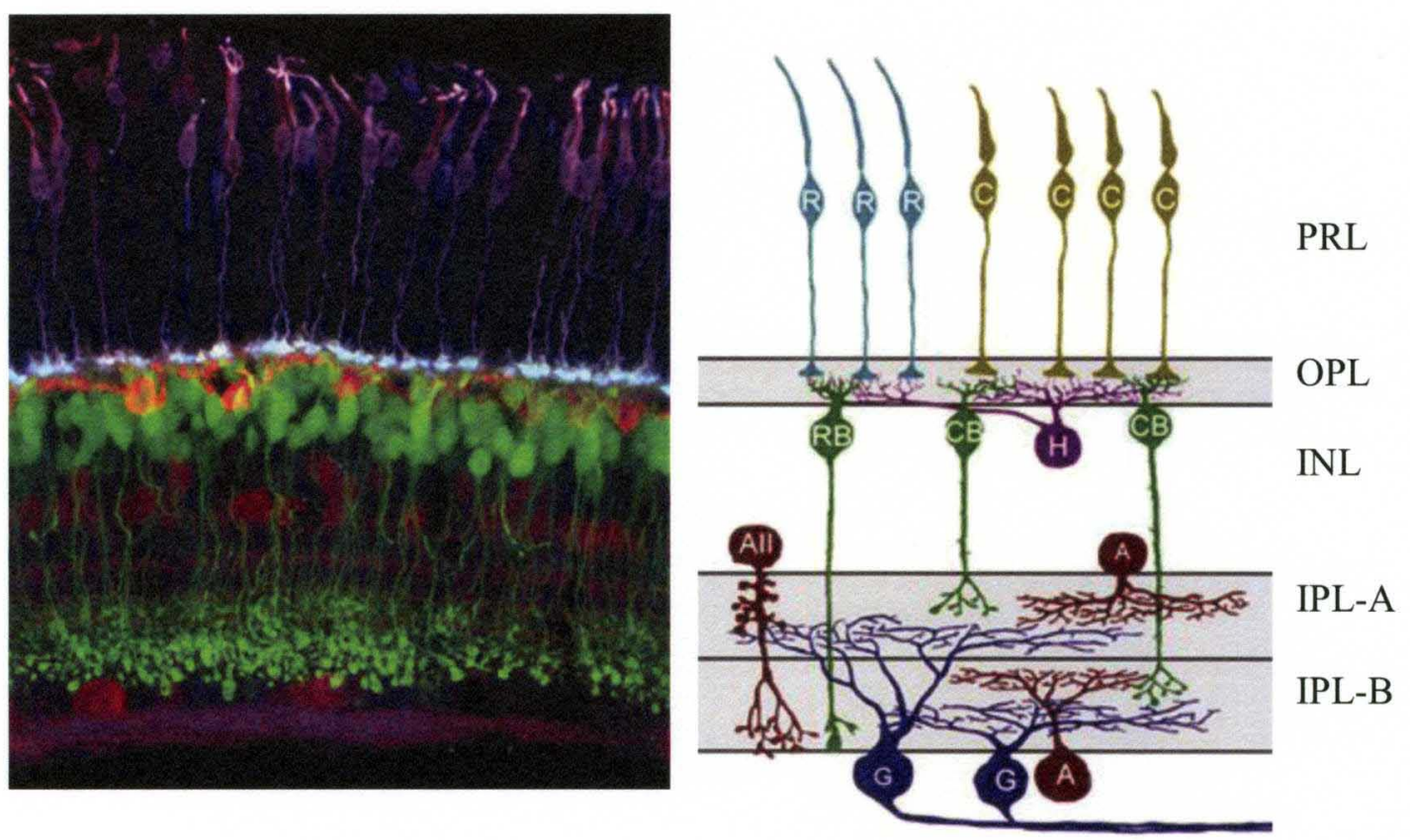
Figure 3. Summary of the rod and cone pathways in the retina. Rod pathway (Left): The rod (R) synapses with the rod depolarizing bipolar cell (rB), signaling begins in the bipolar cell using the Grm6 receptor (Turquoise). The rod bipolar cell synapses on to AII amacrine cell (AII) through iGluRs (green). The AII amacrine cell is electrically coupled to cone depolarizing bipolar cells (cdB) through gap junctions (red) and form glycinergenic synapses (pink) with cone hyperpolarizing bipolar cells (chB) and OFF ganglion cells (off GC). Cone pathway (Right): The cone (C) synapses onto both cone depolarizing bipolar cell (rB) and hyperpolarizing bipolar cells using the Grm6 or iGluR receptors, respectively. The cone hyperpolarizing bipolar cell synapses on to OFF ganglion cells (offGC) while the cone depolarizing bipolar cell synapses on the $\mathrm{ON}$ ganglion cells (onGC). The rod can influence the cone pathway through gap junction. Cells shaded dark grey are depolarized during light decrement, cells shaded tan are excited during light increment and cells shaded light grey are considered not to be directly involved in the pathway. 


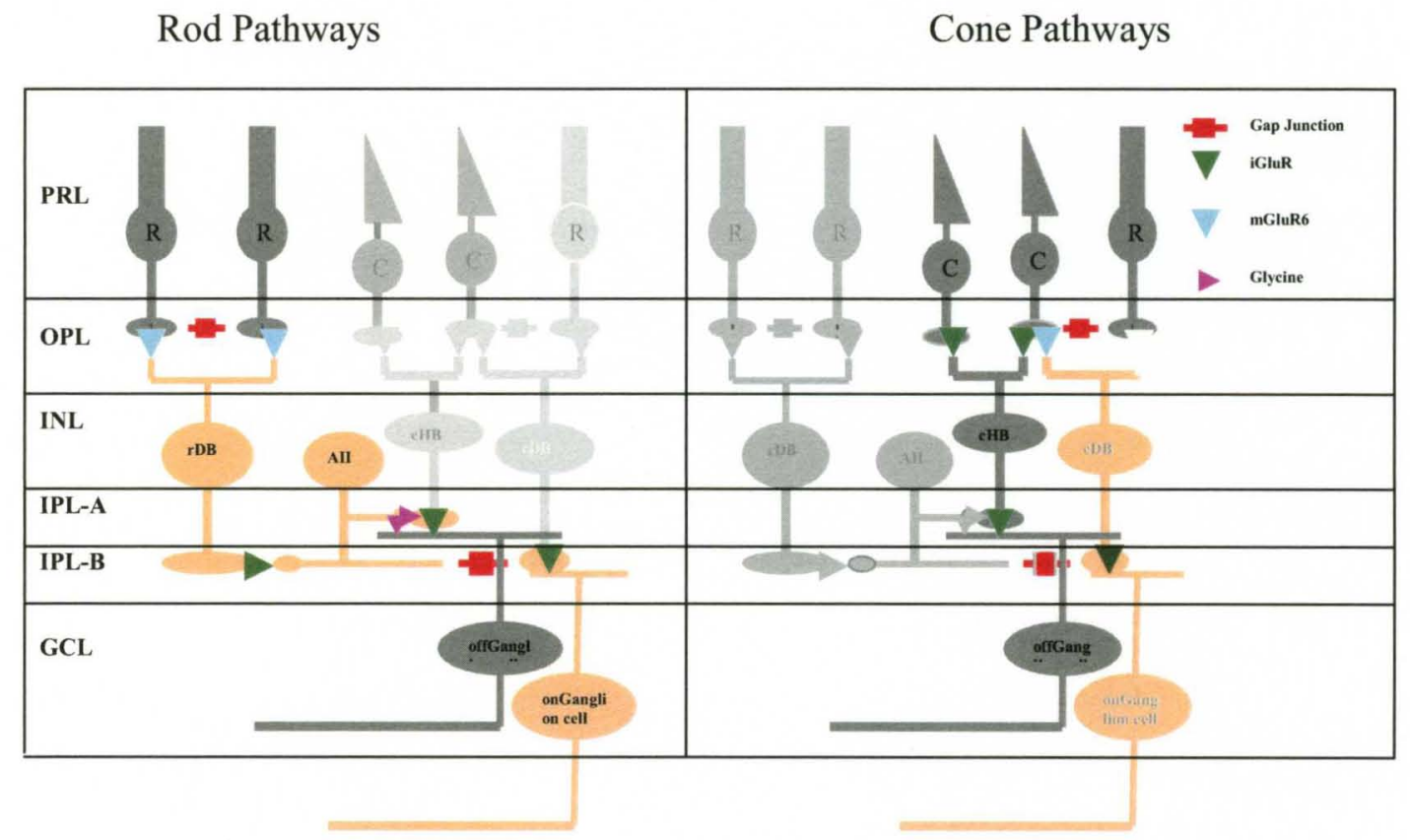


which results in depolarization of depolarizing bipolar cells and hyperpolarization of hyperpolarizing bipolar cells. In addition to the vertical pathways formed by photoreceptors and bipolar cells, horizontal cells provide lateral connections in the OPL between the photoreceptors and bipolar cells. In the IPL, amacrine cells provide lateral connection between the bipolar cells and the ganglion cell inputs. The electrical signal is first segregated into the ON and OFF pathways by the bipolar cells, which is further processed by the lateral connections of amacrine cells. Rod bipolar cells transmit signal vertically to AII amacrine cells, which relay the signal to ganglion cells via cone bipolar cells. Finally, the ganglion cells relay the signal to the brain through the optic nerve. Figure 3 summarizes signal transduction in the retina.

\section{Structure and functional analysis of the outer plexiform layer (OPL) of the retina}

The retina converts light energy into an electrical signal that is transmitted via the optic nerve to the brain for visual processing. Structurally, the retina consists of three distinct layers of neuronal cell bodies and two synaptic layers (Figure 2). The three neuronal cell body layers are photoreceptor layer (PRL), the inner nuclear layer (INL) and the ganglion cell layer (GCL). The two synaptic layers are the outer and the inner plexiform layers ( OPL and IPL). At the base of the retina is the retinal pigment epithelium (RPE). The structural organizations of rod and cone photoreceptors consist of an outer segment, inner segment, a cell body, and an axon with a synaptic terminal (Figure 8A). The outer segment contains the membrane disks that are densely packed with the phototransduction proteins. At the electron microscope level, the outer segment of rod photoreceptor is rod-shaped while that of the cone is cone-shaped (Sjostrand et al., 1953). 
Figure 4. The structure of a ribbon synapse. A. An electron micrograph of a ribbon synapse in the OPL of rat retina immunoreacted for bassoon (dark spots). Bassoon is presynaptic cytomatrix protein, a major component of the photoreceptor ribbon. Photoreceptor ribbons lacking bassoon are not anchored to the presynaptic active zones. Note the localization of bassoon to the presynaptic rod spherule at the active zone. The synaptic ribbon stands perpendicular to the presynaptic membrane and is about $0.5 \mu \mathrm{m}$ long. The dendrite of a horizontal cell is indicated here by $\mathrm{H}$ (Brandstatter et al., 1999). B. A diagramatic representation of the ribbon synapse in the OPL of the retina. The ribbon plate stands perpendicular to the pre-synaptic membrane and is tethered with synaptic vesicles. In addition, this cartoon shows an array of pre-synaptic proteins (piccolo, Rim, bassoon, munc13-1, VDCC, etc) and post synaptic proteins (mGluR (Grm6) and iGluR receptors) (Dieck et al., 2006). 


\section{Ribbon}

A.

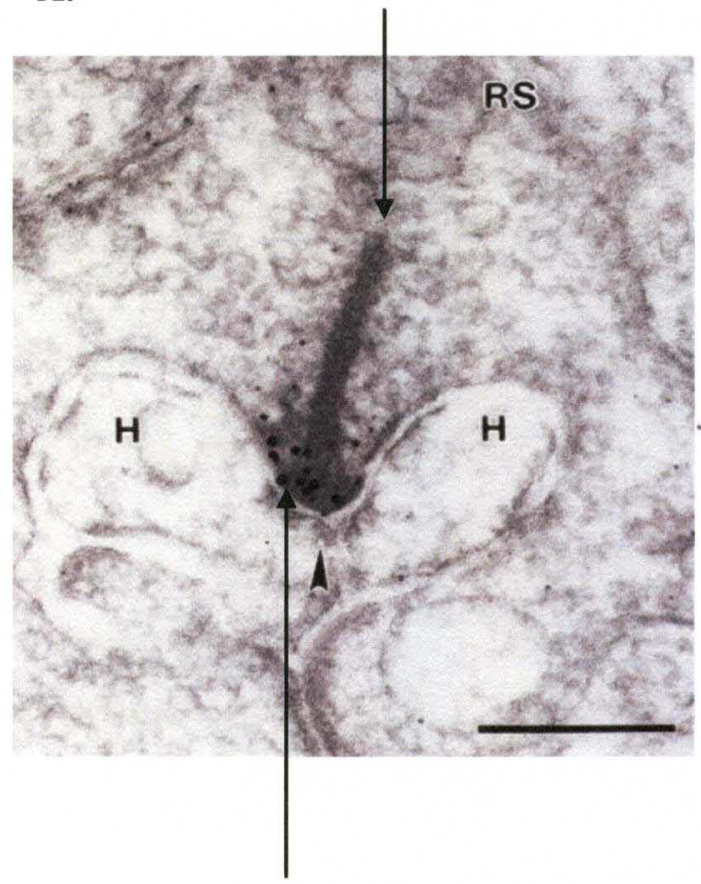

Bassoon
B.

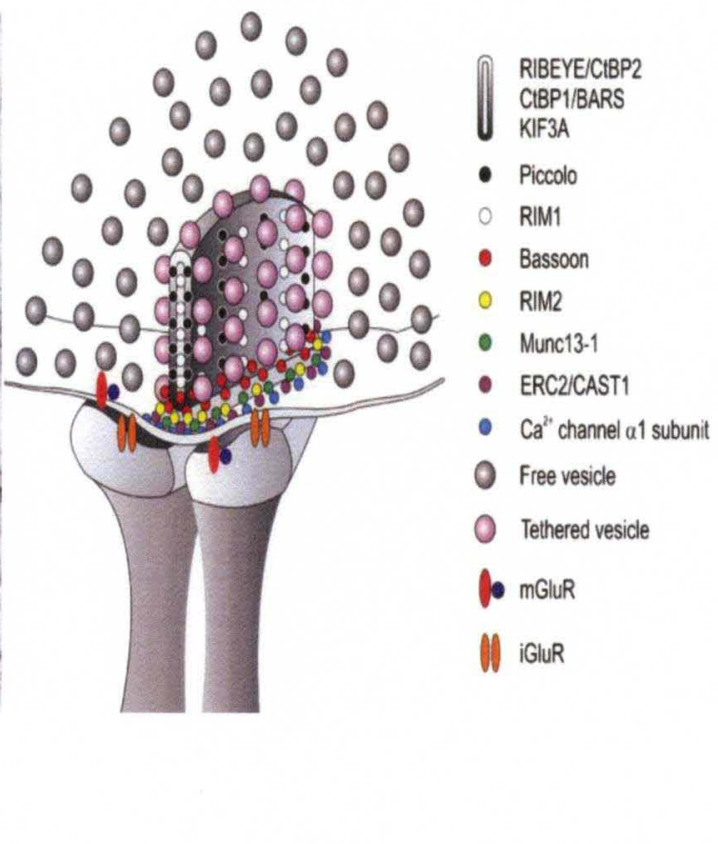


The inner segments contain ribosomes and mitrochondria. Most vertebrates have a single type of rod photoreceptor and this is used to detect light under scotopic conditions (Schulte et al., 2008). Several types of cones exist in most vertebrates (Schulte et al., 2008). Humans are trichromatic so they have S-, L-, and M- cone photoreceptors, which are also called the blue, green and red cones, respectively. They have peak light sensitivity at $420 \mathrm{~nm}, 530 \mathrm{~nm}$ and $560 \mathrm{~nm}$, respectively. Mice are dichromatic and have S- and $\mathrm{M}$ cone photoreceptors (Schulte et al., 2008). The cone photoreceptors are generally used to detect light under photopic conditions. The terminals of the rod and cone photoreceptors are called the rod spherule and cone pedicle, respectively. In the OPL, the rod spherules and cone pedicles connect to the dendrites of bipolar and horizontal cells.

Signal transmission to second order neurons (i.e. bipolar and horizontal cells) occurs at the invaginating ribbon synapses and flat synapses; while signaling from rod to cone or cone to cone occurs through gap junctions in the OPL (Kolb et al., 1977). At the electron microscope level, a ribbon synapse is recognized by a presynaptic dense structure referred to as the ribbon (Figure 4). The ribbon stands perpendicular to the presynaptic plasma membrane. It is about $30 \mathrm{~nm}$ thick and extends about 200 to $1000 \mathrm{~nm}$ away from the active zone (Sjostrand et al., 1953; Sjostrand et al., 1958; see review by Sterling et al., 2004). The ribbon is anchored to the presynaptic membrane by an electron-dense structure called the arciform density.

A large number of synaptic vesicles are tethered to the ribbon, which is thought to act as a conveyor belt to allow multi-vesicular release of neurotransmitter by compound exocytosis (see review Parson and Sterling, 2003). The physiology of the ribbon synapse is different from other synapses in that it releases neurotransmitter continuously and in a 
graded manner (Morgan et al., 1998; Yagi and Macleish, 1994). Flat synapses are located at the base of the cone pedicles. They are found between the cones and hyperpolarizing bipolar cells. There is no evidence of presynaptic densities; rather flat synapses rely on the glutamate spilled from ribbon synapses to mediate signal transmission (DeVries et al., 2006).

On the outer retina gap junctions couple two adjacent rods, cones or a rod to cone photoreceptor. Gap junctions are pores that transmit electrical signals between connected partners (Kolb et al., 1977). Each pore consists of cylindrical hemi-channel hexamer of transmembrane proteins encoded by the connexin family of proteins. The hemi-chemicals in adjacent cells interact to form the pore. Gap junctions link the cytoplasm of one cell to that of the other.

The protein composition at ribbon and flat synapses is different (Ullrich et al., 1994; Brandstatter et al., 1996; Kriegstein et al., 1999). Syntaxin 1 is found at flat synapses and syntaxin 3, B16, and ribeye are found at ribbon synapses (Balkema et al., 1991; Morgans et al., 1996; Schmitz et al., 2000). In addition, ribbon synapses use L-type voltage dependent calcium channel (L-VDCC) to signal neutransmitter release while flat synapses rely on glutamate spillover (Heidelberger et al., 1992; Nachman-Clewner et al., 1999; DeVries et al., 2006).

The INL contains the cell bodies of the bipolar cells, horizontal cells and amacrine cells. Functionally, bipolar cells are divided into two classes: the depolarizing (DBC) or ON bipolar cells and hyperpolarizing (HBC) or OFF bipolar cells (Kolb and Nelson et al., 1995). In response to a light increment glutamate release decreases and the depolarizing bipolar cells depolarize and hyperpolarizing bipolar cells hyperpolarize (Saito and Kaneko, 
1983; Attwell et al., 1987; Nawy \& Jahr, 1991; Masu et al., 1995). The difference in response to glutamate between the two lies on the type of receptor expressed at the tips of their dendrites. Depolarizing bipolar cells express metabotropic glutamate receptors (mGluRs) (Nomura et al., 1994; Masu et al., 1995; Verdi et al., 2000) while the hyperpolarizing bipolar cells express ionotropic glutamate receptors of the AMPA/Kainate type (iGluRs) (Brandstatter et al., 1997; Hack et al., 1999; Devries et al., 2000).

The mGluRs are different from the iGluRs in both structure and function. Each mGluR constitutes a functional receptor; however, they do not form ion channels. They exact their effect by influencing intracellular second messenger signaling cascades via Gproteins. To date, eight mGluRs have been cloned and they are divided into three groups' based on their sequence similarities, pharmacology and second messengers to which they are coupled. The focus of this thesis will be on Grm6, which is expressed exclusively at the dendritic tips of depolarizing bipolar cells (Nomura et al., 1994; Masa et al., 1995; Verdi et al., 2000). Grm6 belongs to the Group-III Grm's and is distinguished pharmacologically from other Grms by its sensitivity to the agonist L-(+)-2-Amino-4phosphonobutyric acid (L-APB) (Nakanishi et al., 1994; Pin and Duvoisin, 1995).

The iGluRs are divided into three groups based on their pharmacology and electrophysiology. The three groups are the $\alpha$-amino-3-hydroxy-5-methyl-4-isozole propanate (AMPA), kainate and N-methyl-D-aspartate (NMDA) receptors. All three types are expressed extensively in both the retina and the central nervous system. Each type has multiple isoforms. Unlike the Grms, the iGluRs can homo- or hetero-dimerize to form selective ion channels. 
Structurally, bipolar cells are divided into rod and cone bipolar cells (Sterling et al., 1995). In human and mouse retinas, there is a single type of rod bipolar cell; while there are ten types of cone bipolar cells (Mariani et al., 1985; Boycott and Wässle, 1991; Euler and Wässle, 1995; Ghosh et al., 2004; MacNeil et al., 2004; Pignatelli and Strettoi, 2004). Rod bipolar cells are depolarizing bipolar type cells and the cone bipolar cells are subdivided into four depolarizing bipolar cells and six hyperpolarizing bipolar cells (Hartveit et al., 1997; Ghosh et al., 2004). The rod bipolar cells make contact with rod photoreceptor through ribbon synapses. The hyperpolarizing bipolar cells makes contact with cone photoreceptors through flat as well as ribbon synapses (Kolb et al., 1970; Dowling et al., 1987; Hopkins et al., 1995) and cone depolarizing bipolar cells forms synapses with cone photoreceptors through ribbon synapses.

Bipolar cells form synapses with amacrine cells and ganglion cells in the IPL. The IPL is divided into two main sub-laminae called sublamina A and B (Cajal $R$ y et al., 1872). This lamination reflects signal segregation into the ON and OFF pathways of the retina (Cohen and Sterling, 1990; Wassle and Boycott, 1991). In the vertical pathways, the cone hyperpolarizing bipolar cells form synapses with OFF ganglion cells in sub-lamina A; while cone depolarizing bipolar cell form synapses with $\mathrm{ON}$ ganglion cells in sub-lamina B (Famiglietti and Kolb, 1976; Nelson et al., 1978). The rod bipolar cells do not directly synapse onto the ganglion cells, instead they form synapses with AII amacrine cells (Famiglietti and Kolb, 1974; McGuire et al., 1984). The AII amacrine cells form glycinergic synapses with cone hyperpolarizing bipolar cells in sublamina A and electrical synapses with cone depolarizing bipolar cells in sublamina B (Famiglietti and Kolb et al., 1975; Manookin et., al 2008), which transmits the rod signal to OFF and ON ganglion cells 
respectively. During light increments, AII amacrine cells receive excitatory input from rod depolarizing bipolar cells and they are depolarized (Dacheux and Raviola et al., 1986; Boos et al., 1993). Depolarization of AII amacrine cell results in depolarization of cone depolarizing bipolar cells via gap junctions or electrical synapses, which leads to the release of glutamate and excitation of ON ganglion cell (Sterling et al., 1998). The excitation of AII amacrine cell also results in release of glycine onto hyperpolarizing bipolar cells and causes their hyperpolarization. There are about 30 different types of amacrine cells involved in lateral inhibitory signaling from the bipolar cell to the ganglion cell. The outermost layer of the retina is the ganglion cell layer that houses the cell bodies of displaced amacrine cells, and ON and OFF ganglion cells.

\section{Assessing signal transduction in the retina using electroretinogram (ERG)}

The electroretinogram (ERG) is a non-invasive method used to assess retinal function (Granit et al., 1947; Riggs et a., 1965; Brown et al., 1965). The ERG is recorded by placing an electrode in contact with the corneal surface and measuring the mass electrical potential in response to light stimulation. The ERG is a measure of the total potential change in the retina in response to light increments and decrements as a function of time after stimulus. Under dark adapted conditions, the ERG measures activity generated by the rod photoreceptor (Figure 5A); while under light adapted conditions, the ERG measures activity generated mainly by the cone photoreceptor (Figure 5B) (see review Hood and Birch, 1996, 1997; Pennessi et al., 1998; Robson and Frishman, 1999). A single flash ERG has two main components in mice, the a-wave and the b-wave (Figure 5). Additional oscillatory potentials (OPs) are superimposed on the b-wave (Figure 5). The 
OPs are generated by the activity of the amacrine cells (Wachtmeister and Dowling, 1978). The negative going a-wave is produced by the hyperpolarization of the photoreceptor outer segments in response to a flash of light; while the positive going b-wave is produced by the subsequent depolarization of depolarizing bipolar cells (Robson and Frishman, 1998; Pugh et al., 1998).

By using APB, an agonist for the Grm6, the ERG b-wave was abolished (Stockton and Slaughter, 1989). Agonists such as kynureic acid or cis-2-3-piperidine dicarboxylic acid have no effect on the ERG $b$-wave. To further tease out the origin of the b-wave, Tian and Slaughter did slow application of APB while simultaneously recording ERGs and intracellular depolarizing bipolar cell recordings on salamander retinas. Results from these experiments show that the two wave forms have strong positive correlation (Tian and Slaughter, 1995). From this experiment and experiments by Robson and Frishman, 1999, the b-wave was confirmed to be generated by the depolarizing bipolar cells.

There is also a c-wave and a d-wave. The c-wave is generated by the pigment epithelilium and the Mueller cells (Noell, 1953). The d-wave is generated by the cone photoreceptor and the Mueller cells (Miller and Dowling, 1970). The d-wave is much more prominent in the cone dominated retinas such as zebrafish and is thought to be generated by the rapid turn off of cone photoreceptors.

Thus, the ERG can be used to detect defects in the retina by comparing the ERG of normal and diseased retinas. In particular, defects in signaling from the photoreceptor to depolarizing bipolar cell can be detected by comparing the amplitudes of the a- and bwaves of the ERG. In this context, the ERG has been used to identify mouse mutants with defects in signaling from photoreceptor to bipolar cell. 


\section{Congenital stationary night blindness (CSNB)}

Congenital stationary night blindness (CSNB) is a group of genetic retinal disorders that are characterized by impaired nocturnal vision of different degrees (Cunier et al., 1838). Patients with this disorder have symptoms that include: myopia, strabismus, reduced visual acuity, nystagmus, and hyperopia. The prevalence of CNSB is approximately 1 in 10000 (Rosner et al., 1993). CSNB is divided into two broad categories based on fundus appearance namely CSNB with normal fundus and CSNB with abnormal fundus (Figure 6) (Cunier et al., 1838; Nettleship, 1907; DeJean et al., 1949; Carr, 1974).

CSNB with abnormal fundus is categorized into the Oguchi type and the fundus albipunctatus type (Oguchi, 1907; Lauber, 1910). Patients with either Oguchi or fundus albipunctatus types of CSNB have difficulty dark adapting (Carr, 1974). The Oguchi type is characterized by a grey-white discoloration of the retina under light condition (Oguchi, 1907). Under prolonged dark adaption, the retina of these patients goes back to normal coloration and when illumination changes the grey-white discoloration reappears (Mizou et al., 1913). This change in discoloration is known as the Mizuo's phenomenon. Oguchi's type of CNSB is characterized by the absence of a b-wave and it follows an autosomal form of inheritance. Fundus albipunctatus is characterized by present of small white spots in the retina with yellow spots located deep in the retina near the pigment epithelium (Lauber, 1910; Fanceschetti and Chome-Bercioux, 1951). The mode of inheritance is autosomal recessive and both rod and cone function are affected in these patients (Carr, 1974). Patients with Fundus albipunctatus disease have a mutation in the RDH5 gene which encodes for the enzyme 11-cis-retinal dehydrogenase (Nakamura et al., 2000). This 
enzyme is involved in the conversion of 11-cis-retinol to 11 -cis-retinal, which eventually leads to the recovery of rhodopsin after light stimulus.

CSNB with normal fundus is categorized into Rigg's type, Naugaret type and Schubert Bornschein type based on the ERG (Schubert and Bornschein, 1952; Riggs, 1954). In Rigg's type, both the a-wave and the b-wave are reduced although the amplitude of the b-wave is larger than the a-wave (Riggs, 1954; Auerbach et al., 1969). Visual acuity tends to be normal or nearly normal in the Riggs type of CSNB. The mode of inheritance for this type of CSNB is unclear.

In Naugaret type of CSNB, both the a-wave and the b-wave are reduced (Cunier et al., 1838; Nettleship, 1907). It follows an autosomal dominant mode of inheritance (Miyake et al., 1987). For example, defects in patients with this type of CSNB have been shown to be caused by missense mutations in either transducin or rhodopsin (Dryja et al., 1993; Seiving, 1995; Dryja et al., 1996). These mutations cause constitutive activation of transducin or rhodopsin even in the absence of light.

In Schubert Bornschein type, the a-wave in the ERG is normal but the b-wave is abnormal (Schubert and Bornschein, 1952). Based on the ERG b-wave, the Schubert Bornschein type of CSNB is further divided into complete and incomplete CSNB (Miyake et al., 1987). In both forms, the ERG a-wave is completely normal but the b-wave is reduced or absent (Figure 7). 
Figure 5. Dark and light adapted ERG of normal mouse retina. A. Single flash ERG indicating activity of the rod pathway. It shows a negative a-wave indicating the hyperpolarization in photoreceptor outer segment. A subsequent b-wave follows indicating the depolarization of depolarizing bipolar cell. The OPs on top of the b-wave indicate activity from the amacrine cells. B. Single light adapted bright flash ERG indicating activity in the cone pathway. It is comprised of a very small negative a-wave follow by a positive b-wave with some OPs. Red arrow denotes the onset of light stimulus. (Adapted from Pardue et al., 1998). 
A.

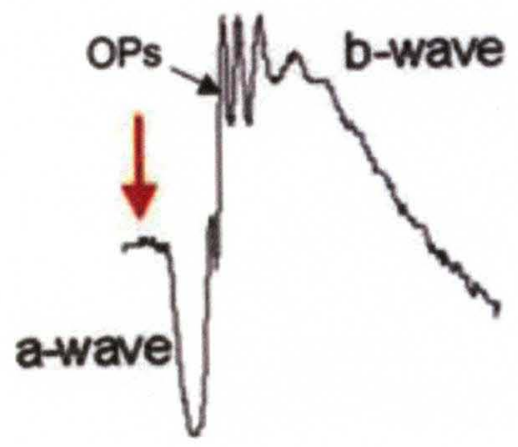

B.

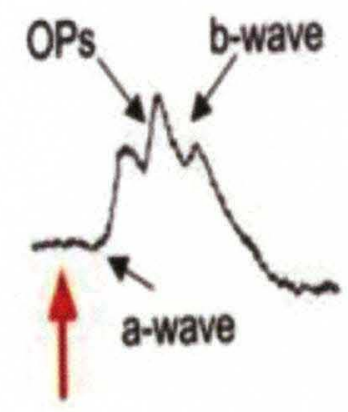


In CSNB1 there is a complete absence of the b-wave. Several animal models with the CSNB1 phenotype have been characterized and all result from defects in signaling

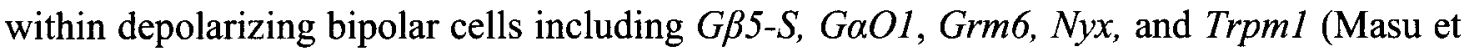
al., 1995; Pardue et al., 1998; Dhingra et al., 2000; Bech-Hansen et al., 2000; Pusch et al., 2000; Gregg et al., 2003; Pinto et al., 2007; Maddox et al., 2008; Bellone et al., 2008; Shen et al., 2009). In most of the mouse models of CNSB1 described to date, the gross morphology of the retina is normal, at both the light and electron microscopic levels (Masu et al., 1995; Dhingra et al., 2000; Pardue et al., 1998, 2001; Ball et al., 2003; Gregg et al 2007) although $G \beta 5$, Grm6 and $G \alpha O$ knockouts, have been shown to have ectopic ribbons in the depolarizing bipolar cells at the electron microscope level (Ishii et al., 2009).

In CSNB2, there is partial loss of the b-wave (Bech-Hansen et al., 1998; Strom et al., 1998; Ball et al., 2002; Haeseleer et al., 2004; Wycisk et al., 2006; Chang et al., 2006). Characterization of several mouse models of CSNB2 shows that the affected proteins are all presynaptic to the bipolar cells and are expressed in photoreceptors. These include

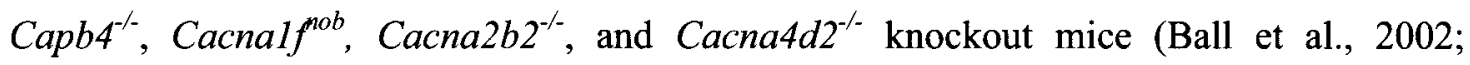
Wycisk et al., 2006; Chang et al., 2006). Unlike CSNB1, the morphology of the retina in CSNB2 mouse models is abnormal at the light microscopic level. In all cases, there is ectopic extension of the dendrites of depolarizing bipolar cells and horizontal cells into the ONL (Ball et al., 2002; Wycisk et al., 2006; Chang et al; 2006). The focus of this thesis will be on the complete form of CSNB (CSNB1) with emphasis on X-linked CSNB1 caused by disruption of the gene encoding nyctalopin. Figure 6 summaries the different types of CSNBs, mutated genes, and the mode of inheritance. 


\section{Signaling in the photoreceptors of the retina}

Light energy is converted into an electrical signal in the photoreceptor outer segments. The photopigment in the outer segment is called rhodopsin. It consists of a chromophore, 11-cis-retinaldehyde, and a protein, opsin. When 11-cis-retinaldehyde absorbs a photon of light, it photo-isomerizes to trans-retinaldehyde (Wald et al 1968). This reaction produces several intermediates that culminate in the formation of metarhodopsin II or opsin without a chromophore. The metarhodopsin II activates a trimeric G-protein complex, transducin, by catalyzing the exchange of GDP for GTP on the $\alpha$-subunit. The exchange of GDP for GTP on the $\alpha$-subunit leads to its dissociation from the $\beta \gamma$ subunit. The GTP bound $\alpha$-subunit then binds to and activates phosphodiesterase (PDE), which hydrolyses cGMP to GMP. The resulting decrease in cGMP levels causes the closure of cGMP gated channels on the outer segments (Figure 8B). Closure of cGMP gated channels hyperpolarizes the photoreceptors outer segment, which leads to the closure of L-type voltage dependent calcium channel (VDCC) at the photoreceptor terminal decreasing the release of glutamate into the synaptic cleft.

After the phototransduction cascade reaches its peak, the signal is terminated in three specific ways. First, transducin $(\mathrm{G} \alpha)$ is inactivated. Transducin $(\mathrm{G} \alpha)$ has an intrinsic GTPase activity that hydrolyses bound GTP. Also Regulator of G-protein signaling protein 9 (RGS9) can regulate and accelerate the GTPase activity of transducin ( $\mathrm{G} \alpha)$ (He et al., 1998). The hydrolysis of GTP on transducin (Ga) reverses its binding to PDE, which inactivates PDE, decreasing the conversion of cGMP to GMP. Second, guanylate cyclase restores the intracellular levels of cGMP to its initial levels in the photoreceptor outer segments (ROS) by catalyzing the conversion of GMP to cGMP. Third, the 
Figure 6. Types of CSNB. The different types of CSNB described base on fundus appearance, mode of inheritance, mutated gene. Schubert-Bornschein type is classified into complete and incomplete type of CSNB base on the amplitude of the b-wave. ArCSNB denotes autosomal recessive mode of inheritance, AdCSNB denotes the autosomal dominant mode of inheritance, and X-linked denote sex linked mode of inheritance. Disrupted genes are in italics and underlined. In the case of SchubertBornschein type the mode of inheritance is in parentheses below the gene 


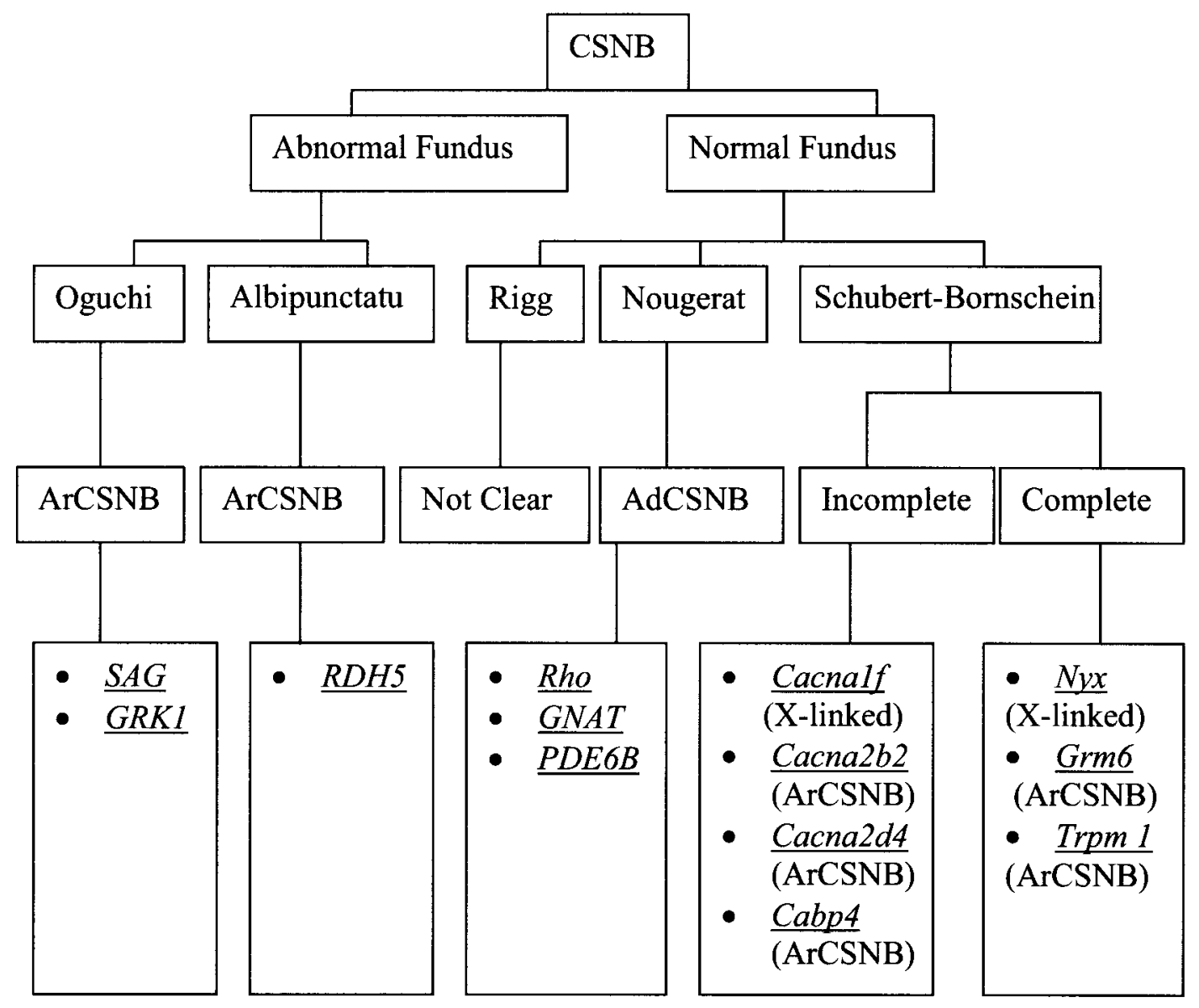


Figure 7. Representative scotopic ERG from three adult males. A. ERG from a normal male of 35 years old. B. ERG from a 66 year old with CSNB1 cause by a mutation in Nyx gene. C. ERG from a 35 year old with CSNB2 cause by a mutation in CACNA1F. Arrows indicate b-wave which is much more reduced in CSNB1 than in CSNB2. The b-wave is abnormal in both cases, but the residual b-wave is more pronounced in CSNB2. (Adapted from Boycott et al., 2010). 


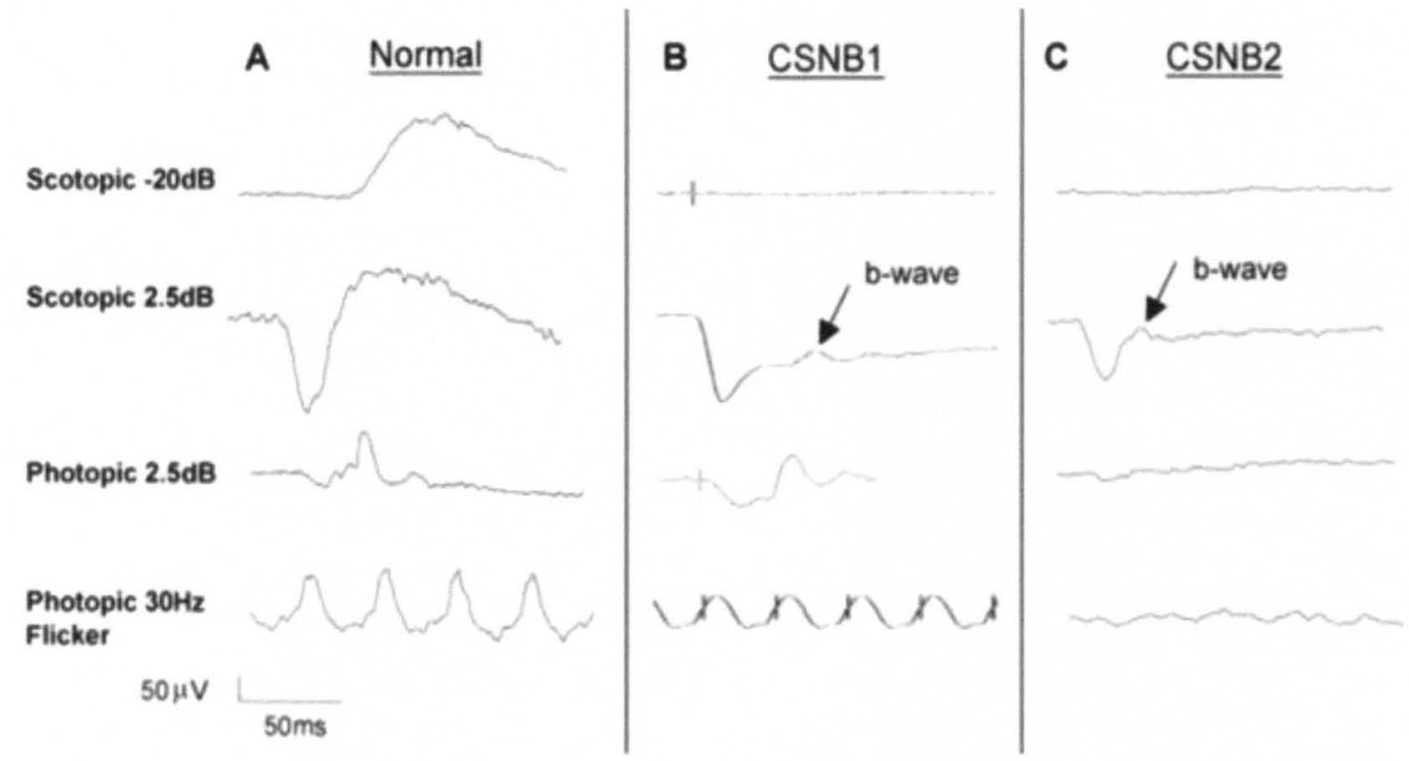


Figure 8. Summary of the phototransduction signaling cascade. A. Shows the rod photoreceptor divided into the ROS, cilium, rod inner segment, cell body and the synaptic terminal. The ROS contain the membrane disks filled with the phototransduction signaling proteins. B. The light activation and inactivation of rhodopsin in the ROS. Light activation of rhodopsin lead to the closure of cyclic GMP gated channel at the membrane of ROS leading to hyperpolarization. Rhodopsin inactivation opens cyclic GMP gated channel leading to depolarization of the ROS. (Modified from Calvert et al., 2006). 
A.

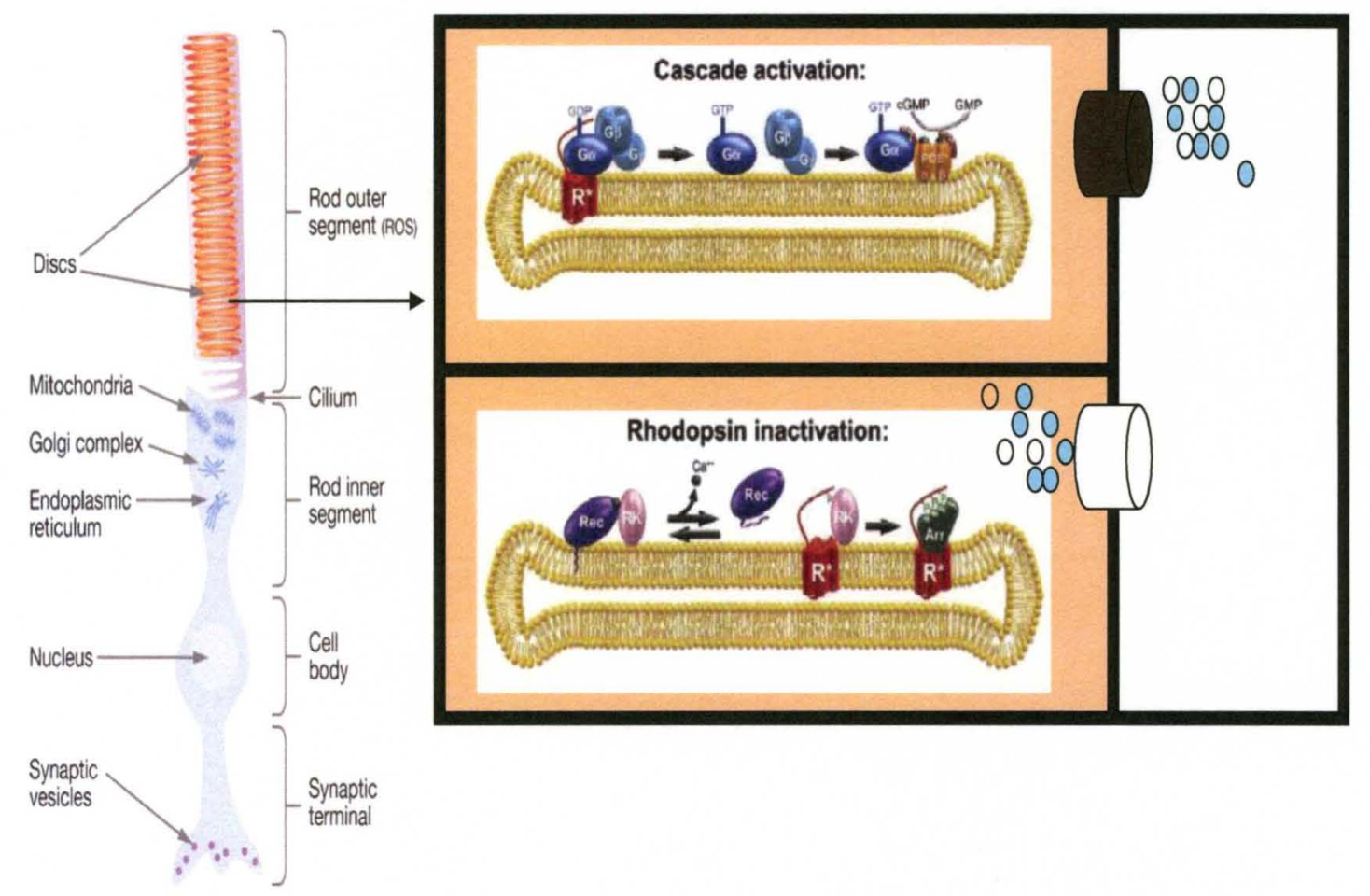


phosphorylation of meta-rhodopsin at three serine residues at the C-terminus that is catalyzed by rhodopsin kinase (Lieberman and Pugh, 1980). Once phosphorylated, metarhodopsin has less affinity for transducin and increased affinity for arrestin (Palczewski et al., 1992). The binding of arrestin to meta-rhodopsin, inactivates meta-rhodopsin. The binding of arrestin to meta-rhodopsin causes trans-retinaldehyde to dissociate from opsin as all-trans-retinal. The all-trans-retinal is reduced to 11 -cis-retinol at the retinal pigment epithelium (RPE) by alcohol dehydrogenase (i.e. prRDH or RDH5) and transported back into outer segment where it is oxidized to 11 -cis retinaldehyde. Finally 11 -cis retinaldehyde recombines with opsin to reform the photopigment ready for a new visual cycle (Figure 8B).

\section{Signaling in the depolarizing bipolar cells}

All photoreceptors are depolarized in the dark, causing a tonic release of glutamate into the synaptic cleft. Hyperpolarizing bipolar cells, which express the ionotropic AMPA/Kainate receptors, depolarize in response to glutamate (Gilbertson et al., 1991; DeVries and Schwartz, 1999; DeVries, 2000). However, depolarizing bipolar cells which express Grm6, hyperpolarize in response to glutamate (Nakajima et al., 1993; Nomura et al., 1994; Vardi et al., 2000). The hyperpolarization of depolarizing bipolar cells indicates the closure of a non-selective cation channel (Schiells and Falk, 1990; Nawy et al., 1991; Yamashita et al., 1991) now known to be Trpml (Bellone et al., 2008; Shen et al., 2009; Morgan et al., 2009; Koike et al., 2010). When there is an increase in light intensity, the photoreceptors respond by decreasing the glutamate released into the synaptic cleft, decreasing Grm6 receptor activity, turning off the G-protein signal cascade 
and allowing the Trpm1 cation channel to open (Figure 9). How the Grm6 receptor gates the Trpm1 channel is poorly understood.

Earlier studies suggested that the Grm6 signaling cascade is similar to the phototransduction cascade in the photoreceptors. Evidence for this came from patch clamp recording of dogfish, salamander and cat depolarizing bipolar cells (Nawy and Jahr, 1990; Shiells and Falk, 1990, 1992; De la Villa et al., 1995; Walters et al., 1998). In those experiments, the authors showed that addition of cGMP to the recording electrode solution elicits an inward current consistent with the closure of the cGMP gated channels. In addition, the mRNA of the cone cGMP gated channel was localized to the terminals of depolarizing bipolar cell in goldfish retina (Henry et al., 2003). In the knockout mice of the cone cGMP gated channel, only the cone mediated pathway is affected while the rod pathway remains intact (Biel et al., 1999). If the Grm6 signal transduction cascade is analogous to the phototransduction cascade, then these data suggest that only cone depolarizing bipolar cells express Grm6; however both cone and rod depolarizing bipolar cell express the Grm6 receptor at their terminals (Nakajima et al., 1993; Nomura et al., 1994; Vardi et al., 2000). In addition, human mutations found in the $\alpha$ - and $\beta$-subunits of cone cGMP gated channel selectively abolished only cone mediated vision (Kohl et al., 1998; Kohl et al., 2000). Finally the response from depolarizing bipolar cells persisted even when a non-hydrolyzable cGMP is added to the recording electrode solution indicating that the channel in depolarizing bipolar cell is not a cGMP gated channel (Snellman and Nawy, 2004). Moreover, the depolarizing bipolar cell response to agonist is not abrogated by inhibitors of PDE (Nawy, 1999). Again, if this system is analogous to the phototransduction system, then inhibitors of PDE should abolish the inward current. 
Glutamate binding to Grm6 results in the activation of a trimeric G-protein

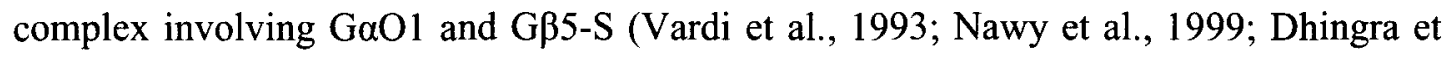
al., 2002; Huang et al., 2003). Several other proteins involved in the modulation of the kinetics of the channel have been identified. These include Regulator of G-protein signaling (RGS), Purkinje cell protein-2 (PCP2 or L7), and nyctalopin.

The G-protein signaling cascade leads to the opening and closing of the Trpml channel, although the details of the cascade are poorly understood. The first step involves the activation of Grm6 by glutamate (Figure 9, Left). Glutamate bound Grm6 is a dimer and each Grm6 subunit consists of seven transmembrane domains with the $\mathrm{N}$-terminus in the extracellular space and the C-terminus in the cytoplasm (Romano et al., 1996). In its inactive form, the Grm6 dimer is coupled to trimeric GaO1ß5S $\gamma$ subunits (Nawy, 1999; Vardi et al., 1993; Mende et al., 1998; Dhingra et al., 2002; Huang et al., 2003). Upon binding to glutamate, Grm6 undergoes a conformational change that results in the exchange of GDP for GTP on the GaOl subunit (step 2). This leads to the dissociation of the trimeric G $\alpha \mathrm{O} 1 \beta 5 \mathrm{~S} \gamma$ complex from Grm6. The GTP bound $\mathrm{G} \alpha \mathrm{O} 1$ dissociates from

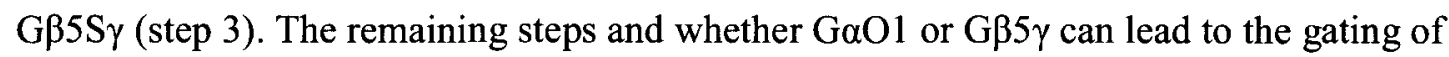
the Trpm1 channel is unknown (step 4).

The involvement of $\mathrm{GaO} 1$ in the Grm6 signaling cascade in depolarizing bipolar cells came from work done by Vardi, Nawy, and Weng and colleagues. When $\mathrm{G} \alpha \mathrm{O}$ is dialyzed onto depolarizing bipolar cells this prevents a glutamate response. Antibodies against $\mathrm{G} \alpha \mathrm{O}$ also reduced the glutamate response (Nawy, 1999). Weng et al., 1997 showed that recombinant Grm6 is able to activate $\mathrm{G} \alpha \mathrm{O}$ 18-times more efficiently than transducin. Later it was shown that a special splice form of $\mathrm{G} \alpha \mathrm{O}, \mathrm{G} \alpha \mathrm{O} 1$, is expressed in 
depolarizing bipolar cells including the dendrites (Vardi et al., 1993; Dhingra et al., 2002; Huang et al., 2003). Finally, knockout mice of $\mathrm{G} \alpha \mathrm{O} 1$ have no ERG b-wave, indicating a defect in the Grm6 signaling cascade (Dhingra et al., 2000). Despite all this, it is not known which protein (s) GaOl binds to, or how and if it is the one that gates the Trpm1 channel.

The first evidence of the involvement of $\mathrm{G} \beta 5-\mathrm{S}$ came from $\mathrm{G} \alpha \mathrm{O}$ knockout mice. Mende et al., 1998 showed that loss of $\mathrm{G} \alpha \mathrm{O}$ led to a $30 \%$ reduction in $\mathrm{G} \beta 5$. Immunohistochemical analyses showed that a splice form of $\mathrm{G} \beta 5, \mathrm{G} \beta 5-\mathrm{S}$, is expressed in the depolarizing bipolar cell including the dendrites and when complexed with RGS proteins, they co-localized with Grm6 (Watson et al., 1996; Zhang et al., 2003; Morgans et al. 2007; Cao et al., 2008; Chen et al., 2009). Furthermore knockout mice of Gß5-S have a no ERG b-wave (Rao et al., 2007).

The fifth step of the cascade occurs when light intensity increases, and glutamate release by the photoreceptor decreases (Figure 9, right). The intrinsic GTPase acceleration protein (GAP) activity in GaOl hydrolyses the bound GTP (step 6). It is now known that this GTP hydrolysis is facilitated by RGS proteins. Recently RGS11 was shown to have GTPase activity when complexed with G $\beta 5-S$ and a R7 family binding protein (R9AP). This complex promotes GTP hydrolysis on the GaO1 subunit (Masuho et al., 2010; Coa et al., 2009). This inactivates $\mathrm{GaO} 1$ and accelerates depolarizing bipolar cell light responses. The ERG b-wave in the RGS11 knockout mice is present but delayed suggesting an important role for GAP activity (Chen et al., 2010).

The seventh step involves the reformation of the GDP bound trimeric GaO1ß5S $\gamma$ complex, which relieves the signal that closes the Trpm1 channel. Consequently the 
Trpm1 channels open leading to depolarization of depolarizing bipolar cells. Purkinje cell protein-2 (PCP2) knockout mice have a normal ERG a-wave but a slower falling phase of the b-wave suggesting a delay in closing of the Trpml channel (Xu et al., 2008). Loss of PCP2 results in the delay of GTP/GDP exchange and increases the fraction of Trpm1 channel open under light conditions (Xu et al., 2008). In dark adapted conditions, PCP2 can facilitate the closing of the Trpm1 by either acting as a guanine nucleotide exchange factor (GEF) or an inhibitor of GDP dissociation (GDI) (Xu et al. 2008) (Step 2). As a GEF, PCP2 can activate both $\mathrm{G \alpha O} 1$ and G $\beta 5-\mathrm{S}$, which means both can potentially gate

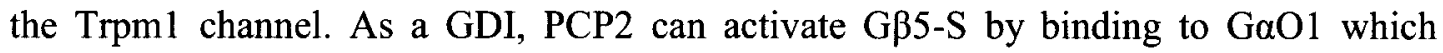
would suggest that Trpm1 is gated by G $\beta 5$-S. However a recent paper by Koike et al. 2009 shows that ionic current were suppressed in Chinese hamster ovary (CHO) cells expressing Grm6, Trpml and $\mathrm{G} \alpha \mathrm{O}$ suggesting that Trpml channel is gated by $\mathrm{G} \alpha \mathrm{O}$. In all, it is still not clear how and what gates the Trpml channel or what additional molecules are required for Trpm1 channel gating.

Pardue et al. 1998 identified a mouse mutant that lacks the b-wave in the ERG. The gene responsible for the defect was subsequently identified in mice as $N y x$, which encodes the protein called nyctalopin (Gregg et al., 2003). A clue to the function of nyctalopin in depolarizing bipolar cells came from patch clamp recordings bipolar cells from $N y x^{n o b}$ and control littermates. When glutamate was puffed onto hyperpolarizing bipolar cells from $N y x^{n o b}$ and control, both responded with a robust transient inward current, which indicated the opening of AMPA/ kainate receptors (Gregg et al., 2007). Glutamate puffed on to wild type control depolarizing bipolar cells showed a robust outward current indicating the closure of the non-selective cation channel. However when 
Figure 9. Summary of the signal transduction cascade in the depolarizing bipolar cell. (Left) In the dark, there is a tonic release of glutamate into the synaptic cleft. The glutamate binds to the Grm6 receptor, which leads to the exchange of GDP for GTP on the $\mathrm{G} \alpha \mathrm{O}$ subunit of the trimeric G-protein $(\mathrm{G} \alpha \beta \gamma)$. Through GEF activity, PCP2 can increase the rate of exchange of GTP for GDP on the GaO subunit. The trimeric G-protein then

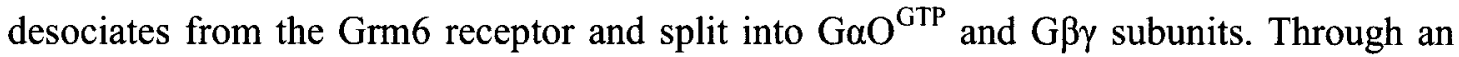
unknown mechanism, either $\mathrm{G}_{\alpha} \mathrm{O}^{\mathrm{GTP}}$ or $\mathrm{G} \beta \gamma$ can lead to the closure of the Trpm1 channel at the plasma membrane. These series of events cause the depolarizing bipolar cell to hyperpolarize. (Right) During light increment, glutamate release into the synaptic cleft decreases leading to reduced Grm6 receptor activity. The GTP on the $\mathrm{GaO}^{\mathrm{GTP}}$ is hydrolyzed into GDP. GTP hydrolysis is enhanced by RGS proteins (RGS7 or RGS11) and the GTPase activity of $\mathrm{G} \alpha \mathrm{O} . \mathrm{G} \alpha O^{\mathrm{GDP}}$ and $\mathrm{G} \beta \gamma$ bind to re-constitute the trimeric $\mathrm{G}$ protein which re-binds to the Grm6 receptor. The Trpml channels open causing the depolarizing bipolar cell to depolarize. PCP2 can enhance the reconstitution of $\mathrm{G} \alpha \mathrm{O}^{\mathrm{GDP}}$ and $\mathrm{G} \beta \gamma$ by binding to $\mathrm{G} \alpha \mathrm{O}^{\mathrm{GDP}}$. 


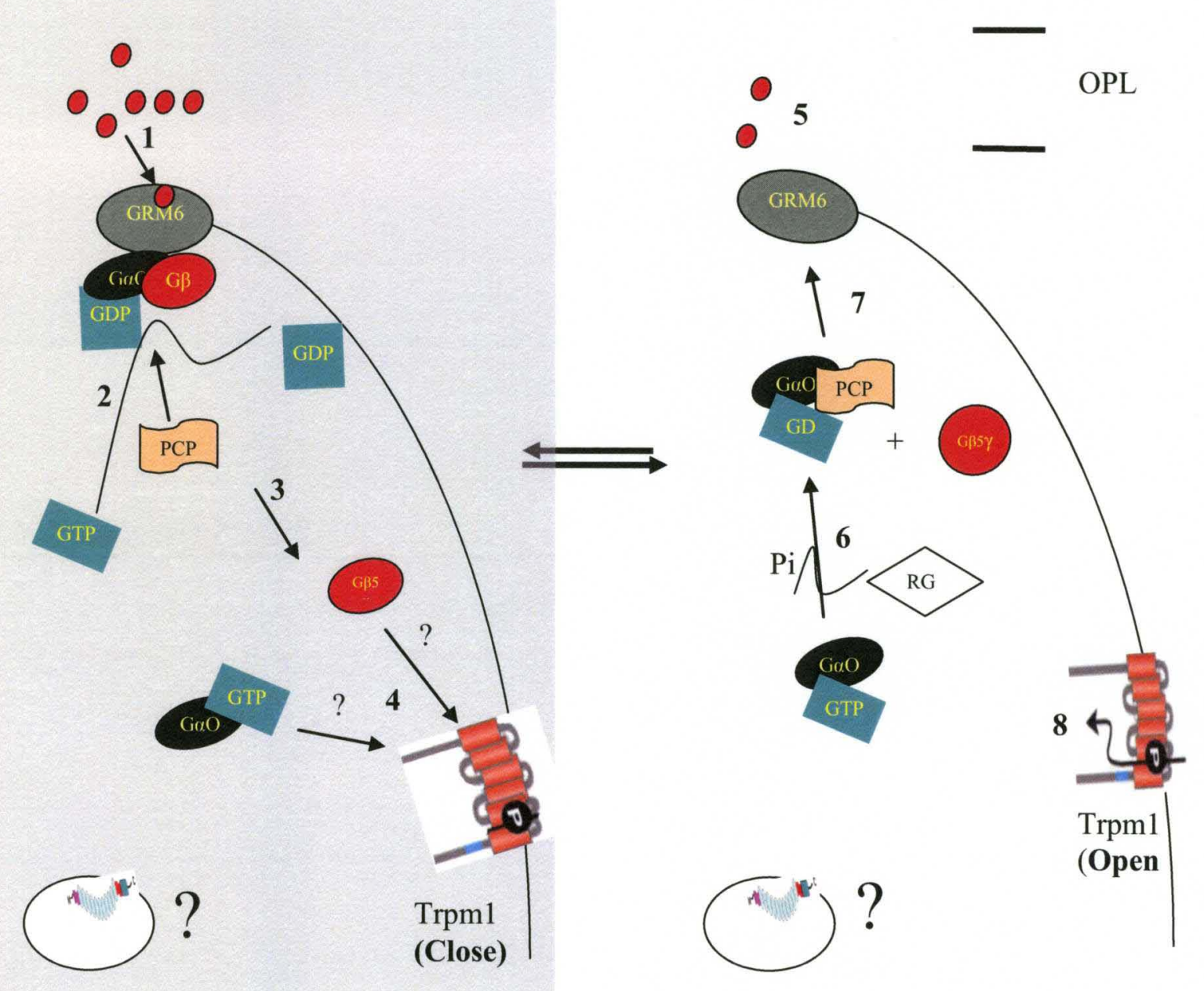

Dark

Light 
glutamate was puffed onto depolarizing bipolar cells from $N y x^{n o b}$, the outward current is absent. Using noise analysis, the authors determined that the open probability of the channel in the $N y x^{n o b}$ depolarizing bipolar cells was very low. Together, these data suggested that in the absence of nyctalopin, the non-selective cation channel is closed or absent in $N y x^{n o b}$ depolarizing bipolar cells. Nyctalopin might be involved in the trafficking of Trpml channel to the membrane.

\section{Structure of nyctalopin and other possible functions}

Nyctalopin belongs to a family of small leucine rich proteoglycans (SLRPs). A total of 17 genes are in the SLRP superfamily (Schaefer et al., 2008). The SLRP superfamily is further divided into five sub-families or classes based on homology at both the genomic and protein level, number of leucine rich repeats (LRR), the presence of $\mathrm{N}$ terminal cysteine rich clusters and chromosomal organization (Goff and Bishop, 2007; Schaefer et al., 2008). A typical SLRP consists of a core protein made of tandem LRRs, which are flanked at the C-terminus and $\mathrm{N}$-terminus by cysteine clusters. Some of the SLRPs carry glycosaminoglycan (GAG) side chains of diverse composition; others like nyctalopin carry an O-linked glycosylation site (O' Connor et al., 2005). A LRR consists of tandem leucine rich repeats and other hydrophobic residues and is about 20-29 amino acids long. Each repeat in the LRR consists of eleven amino acids with a consensus motif of LxxLxLxxNL where $\mathrm{L}$ can be leucine, valine or isoleucine, $\mathrm{N}$ is asparagine, cysteine or threonine and $\mathrm{x}$ is any amino acid (Kajava et al., 1998; Le Goff et al., 2007). A typical LRR is folded into $\beta$-sheets and $\alpha$-helices connected by loops (Figure 10). This arrangement of $\beta$-sheets and $\alpha$-helices gives the tandem LRR domain a horseshoe or 
banana shaped structure with parallel $\beta$-sheets lining the concave side of the horseshoe and $\alpha$-helices lining the convex side. The concave surface of SLRPs is believed to provide a surface for protein-protein interaction.

Nyctalopin's structure is characterized by 11-leucine rich repeats (LRR) that are capped at the N- and C-terminus by a cysteine rich LRR. Computer analyses predict nyctalopin has a signal sequence at the N-terminus. The LRR domains are followed by a GPI anchor in human nyctalopin, which is predicted to be absent in mouse nyctalopin (Gregg et al., 2003). Murine nyctalopin may have as many as two transmembrane domains (Gregg et al., 2003). The crystal structures of three of the family members, biglycan, opticin and decorin, have been determined (Scott et al., 2004). These data indicate that they can homodimerize through their concave side. Since it is the concave side that is involved in interaction, dimerization could mean that functional nyctalopin is a dimer.

The absence of nyctalopin in $N y x^{n o b}$ mouse also may have a secondary effect on gene expression. During normal postnatal retinal development, spontaneous activity in the form of retinal waves is prominent until eye-opening (P11-P15), when waves cease. In $N y x^{n o b}$ mice, in which signaling is absent in depolarizing bipolar cells, abnormal spontaneous activity is maintained into adulthood. These defects in $N y x^{n o b}$ mice are correlated both with abnormal visual responses of retinal ganglion cells and an aberrant segregation of their axonal projections to the dorsal lateral geniculate nucleus (dLGN) (Demas et al 2006). Retinal waves have been shown to be involved in neuronal pathfinding, survival, and the refinement or sculpting of visual maps. Retinal waves are required to maintain eye specific segregation of axonal projections to the dLGN (Chapman et al, 2000). 
Retinal waves are divided into three stages; stage I waves start at about embryonic day 16 and last until postnatal day 0 (P0). These waves can be blocked by gap junction antagonists. Stage II waves start at about postnatal day 0 and last until postnatal day 10 . These are blocked by nicotinic acetylcholine receptor antagonists. Stage III waves occur between postnatal day 11 and postnatal day 15 , and are blocked by ionotropic glutamate receptor antagonists. The transition between stage II and stage III waves occurs when the bipolar cell axons begin to make contact in the OPL and light responses are first detected in retinal ganglion cells (Fisher et al., 1979; Tian, 2004; Demas et al., 2006; Morgans et al., 2007). After this transition, stage III waves persisted until postnatal day 15 when they ceased. In $N y x^{n o b}$ mice, the stage III waves persisted atleast to P28. Expression of a fusion protein EYFP-nyctalopin at the tips of bipolar cells in $\mathrm{Nyx}{ }^{\text {nob }}$ mice was able to rescue this phenotype (Demas et al 2006). These data strongly argue that abnormal retinal waves are caused by loss of nyctalopin in the OPL. An important question is, does loss of nyctalopin and the resulting abnormal electrical activity have an effect on gene expression?

Nyctalopin might also function as part of a complex that is involved in the trafficking of the Trpm1 channel to the membrane. The Trpm1 channel is a non-selective channel that allows sodium and calcium ions to flow into the depolarizing bipolar cell, resulting in their depolarization (Shen et al., 2009; Morgan et al., 2009; Koike et al. 2009). Since calcium overload is detrimental to cells, this would implicate that the localization of Trpml to and from the plasma membrane has to be regulated. Given that the entire LRR domain of nyctalopin is in the extracellular space and it interacts directly with Trpm1, it might be that nyctalopin is part of a complex that traffics Trpml to the plasma membrane. 


\section{CHAPTER II}

\section{THE TOPOLOGY AND HOMODIMERIZATION OF NYCTALOPIN}

\section{INTRODUCTION}

Murine $N y x$ consists of three exons and encodes a 476 amino acid protein with a predicted molecular weight of $52.4 \mathrm{kDa}$ (Gregg et al., 2003). The core of nyctalopin consists of eleven leucine rich repeats (LRR) that are capped at the $\mathrm{N}$-terminus and the $\mathrm{C}$ terminus by cysteine rich motifs. The consensus LRR is 24 amino acids with the sequence, $x-x-I / V / L-x-x-x-x-F / P / L-x-x-L / P-x-x-L-x-x-L / I-x-L-x-x-N-x-I / L$, where $x$ is any amino acid (Kajava et al., 1998; Le Goff et al., 2007). The N-terminus and the Cterminus caps have a consensus arrangement of $\mathrm{Cx}_{3} \mathrm{Cx}_{3} \mathrm{CxCx}_{6} \mathrm{Cx}_{3} \mathrm{C}$ and $\mathrm{CCx} \mathrm{Cx}_{19} \mathrm{Cx}_{23} \mathrm{C}$, respectively (Bech-Hansen et al., 2000; Gregg et al., 2003; Zeitz et al., 2005; McEwen et al., 2006). Each tandem LRR domain is folded into $\beta$-sheets and $\alpha$-helices joined by loops. This arrangement of $\beta$-sheets and $\alpha$-helices gives the tandem LRR domain a horseshoe or banana shaped structure with parallel $\beta$-sheets lining the concave side of the horseshoe and $\alpha$-helices lining the convex side (Figure 10). At the N-terminus of nyctalopin there is predicted signal sequence. At the C-terminus of human nyctalopin there is a consensus sequence for addition of a glycosylphosphatidylinositol (GPI) anchor. However, in mouse this site appears to be absent, rather there may be one or more transmembrane domains (Gregg et al., 2003; O'Connor et al., 2005). When 
Figure 10. Tertiary structure of nyctalopin in a banana conformation. The convex side consists of parallel $\beta$-sheets and the concave side consists of $\alpha$-helices. The $\beta$-sheets and $\alpha$-helices are folded to form 11 tandem leucine rich repeats, which are capped at the $\mathrm{N}$ - and $\mathrm{C}$-termini by cysteine rich repeats. The $\mathrm{N}$-terminus has a predicted signal sequence and the $\mathrm{C}$-terminus has one or more predicted transmembrane domains. 

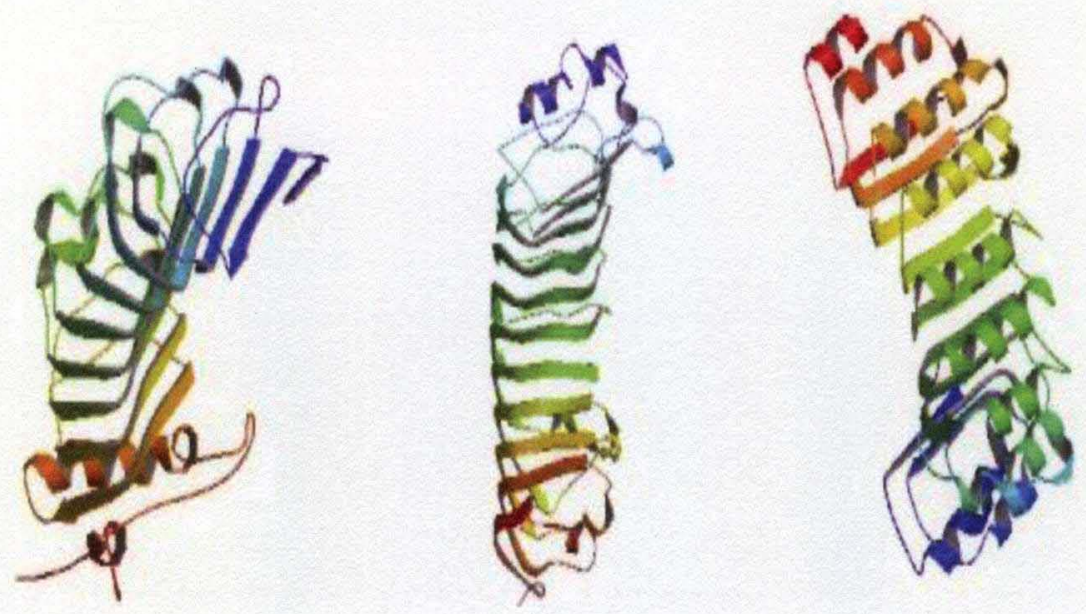
expressed in heterogeneous expression systems, both human and murine nyctalopin were determined to be anchored to the cell surface (Zeitz et al., 2003; O'Connor et al., 2005). Phosphatidylinositol-phosphalipase D (PI-PLD), which specifically cleaves GPI anchors, was able to release human nyctalopin from the cell surface but was not able to release mouse nyctalopin (O'Connor et al., 2005). In addition, hydrazine, which is an inhibitor of GPI cleavage and forms complexes with GPI anchored proteins, does not complex with murine nyctalopin ( $\mathrm{O}^{\prime}$ Connor et al., 2005). These data suggest that murine nyctalopin is anchored to the cell surface differently than human nyctalopin.

The topology of eukaryotic integral membrane proteins is determined at the endoplasmic recticulum (ER) and the process involves at least four steps: ER targeting, translocation initiation, translocation termination, and membrane integration. A signal sequence or a transmembrane domain is involved in the targeting of proteins to the ER membrane. Signal sequences contain a relatively short stretch of hydrophobic amino acids. Transmembrane domains are more hydrophobic and their orientation in the membrane depends on the sequence of the protein.

A typical signal sequence consists of a characteristic tripartite structure made up of N-terminal n-region, central h-region and a C-terminal c-region (Von Heijne et al., 1985). The h-region consists of 7-15 hydrophobic amino acids that determine the targeting pathway to the ER membrane. The c-region consists of 2-9 polar and nonpolar amino acids that determine the cleavage site by signal peptidase (Von Heijne et al., 1990). The $n$-region can range from 2 to 50 amino acids and is the most diverse region (Martoglio et al., 1998) and is believed to determine the rate of cleavage of the signal sequence (Lipp and Dobberstein, 1986; Li et al., 1994). Computer predictions indicate the 
n-region of nyctalopin contains 2 amino acids, the h-region 14 amino acids and the cregion 4 amino acids.

Targeting of membrane proteins to the endoplasmic reticulum can be either co- or post translationally mediated (von Heijne et al., 2002; Villar et al., 2002; Johnssonsson et al., 2002). Co-translational targeting is mediated by the ribonucleoprotein complex (RNC), the signal recognition particle (SRP) and its cognate membrane-associated receptor (SR) located on the ER. Post translational targeting does not involve the RNC or the SRP but rather hydrophobic regions of the protein interact directly with the SR (see review Egea et al., 2005). However in yeast, both post and co-translation targeting are used extensively (see review Rapoport et al., 1996).

During co-translational targeting to the ER, the signal recognition particle (SRP) recognizes and binds to the signal sequence of a nascent protein as it exits the ribosome forming a ribosome nascent chain complex (SRP-RNC) (Walter et al., 1981) (Figure 11). This halts translation and the SRP-RNC translocates to the ER membrane and binds to the signal recognition particle receptor (SR), (Gilmore et al., 1982; Sanders et al., 1992). The nascent protein is inserted into the translocon and translation resumes (Lyko et al., 1995; Panzner et al., 1995; Sanders et al., 1992). Both SRP and SR have GTPase activities and the interaction of the two causes GTP hydrolysis, which governs docking, release of the RNC to and from the translocon and the recycling of the SRP (Connolly et al., 1989; Connolly et al., 1991; Freymann et al., 2000). As translation continues and the protein enters the lumen of the ER, the signal sequence is inserted into the membrane with the $\mathrm{N}$-terminus extending into the cytoplasm (Figure 11). Once inserted into the membrane, the c-region becomes accessible and maybe cleaved by a signal peptidase. For 
Figure 11. The SRP-mediated co-translational targeting of protein through the ER. When the signal sequence of a nascent polypeptide emerges from the ribosome, it is recognized and bound by the signal recognition particle (SRP) halting translation (1). The RNC-SRP complex is then translocated to the ER membrane through GTP-dependent interactions between the SRP and its cognate signal receptor (SR) (2). At the membrane, after ribosome docking to the translocon, the signal sequence is released from the SRP (3) and, following GTP hydrolysis, the SRP-SR targeting complex dissociates (4). The nascent protein is inserted into the translocon and translation resumes. (Egea et al., 2005) 


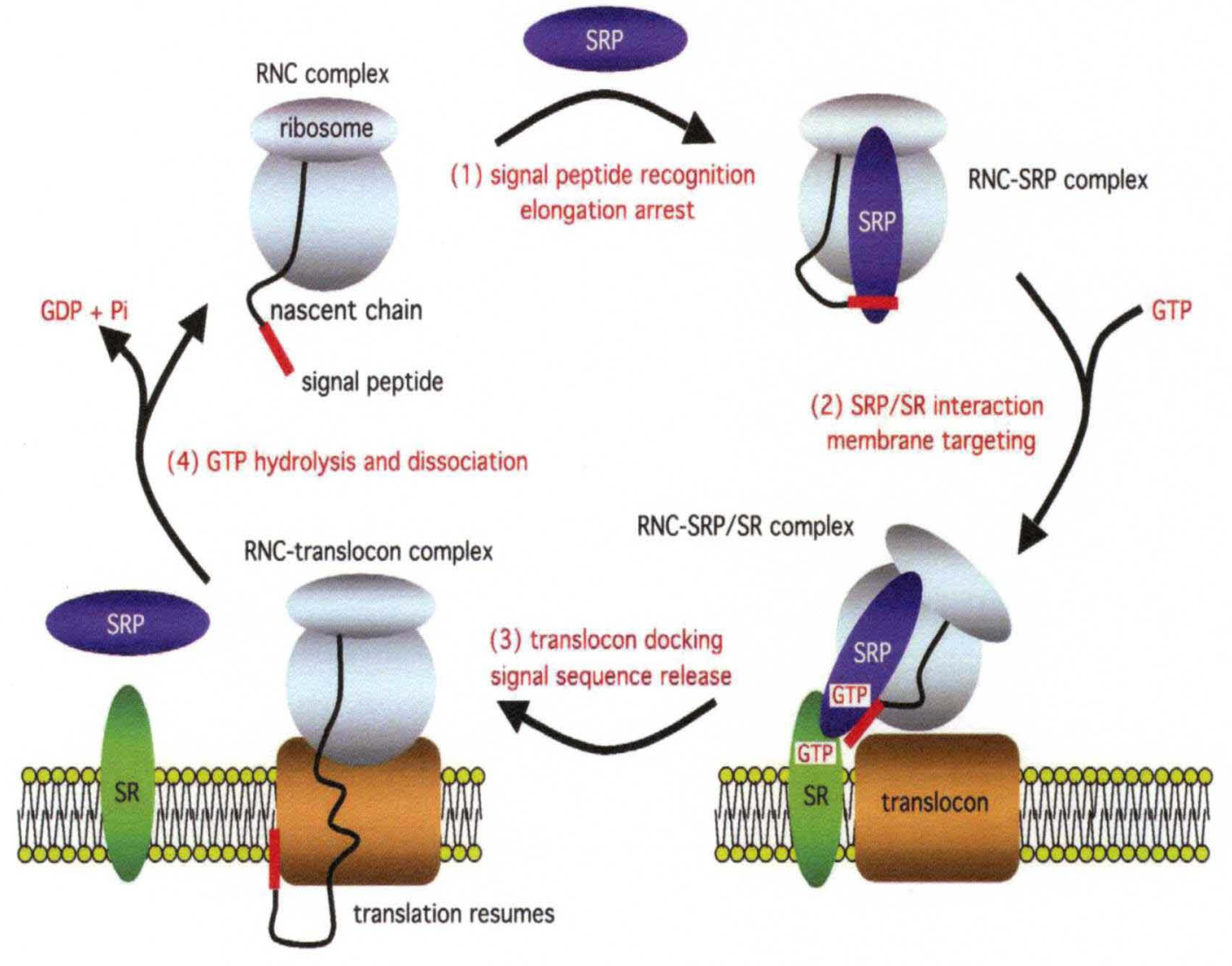


proteins destined to the membrane, translation continues until the first transmembrane domain is synthesized. The orientation in the membrane of the first transmembrane domain is determined by three factors. First, is the folding characteristic of the Nterminus. Proteins with stable N-terminal tertiary structures tend to stay in the lumen of the ER because they are too large to traverse the translocon (Denzer et al., 1995). Second, the charge distribution either before or between transmembrane domains is important (Von Heijne et al., 1986; Hartmann et al., 1989). If the region is positively charged then the intermembrane region tends to remain in the cytosol. Third, longer hydrophobic regions favor localizing the N-terminus in the lumen of the ER (Sakaguchi et al., 1992; Wahlberg et al., 1997).

Proteins are inserted into the ER membrane either as type I or type II membrane proteins. Type I membrane proteins are oriented with the $\mathrm{N}$-terminus in the extracellular space while Type II membrane proteins are oriented with the $\mathrm{N}$-terminus in the cytoplasm. Type I membrane proteins are integrated into the membrane in three stages: First, the transmembrane domain is synthesized and enters the translocon. Second, the region before the transmembrane domain moves toward the cytoplasm, which causes the transmembrane domain to stand perpendicular to the lipid bilayer with the $\mathrm{N}$-terminus facing the lumen of the ER. Third, the transmembrane domain moves sideways through the wall of the translocon into the lipid bilayer. Type II membrane protein orientation also is determined in three stages: First, the transmembrane domain is synthesized and is inserted into the translocon. Second, the region before the transmembrane domain moves into the lumen of the ER forming a loop with the N-terminus inserted into the translocon. This orients the N-terminus to the cytoplasmic side of the ER. Third, the transmembrane 
domain moves sideways through the wall of translocon into the lipid bilayer (see review Rapoport et al., 2004). Once translation and membrane insertion is complete in the ER, the proteins are sorted and transported to the appropriate sub-cellular compartment using a complex series of events that occur in the Golgi network.

Trafficking of the proteins from the ER to the Golgi relies on the coatomer protein complex II (COPII) and the adaptor protein (AP) - clathrin complexes (AP-clathrin complexes). Transport of proteins from the Golgi to the plasma membrane or the endosomes is done by vesicle budding of the Golgi and fusing to the plasma membrane (see reviews Rothman et al., 1994; Lee et al., 2004; Pearse et al., 1990; Ira and Nelson, $2008)$.

Nyctalopin is a member of the small leucine rich proteoglycan (SLRP) superfamily. Family members have diverse membrane orientation and sub-cellular localizations which reflect their functional diversity. Further, some members such as the TrK family of nuclear receptors have been shown to be intercalated into the membrane (Windisch et al., 1995). Others, like Drosophila connectin, are GPI anchored and the ribonuclease inhibitors are localized to the cytoplasm (Meadows et al., 1994, Kobe and Deisenhofer, 1994).

Solution X-ray scattering experiments of two SLRPs decorin and biglycan show they are dimers in solution (Liu et al., 1994; Scott et al., 2003; Scott et al., 2004; Scott et al., 2006). Crystal structures of both proteins show that they form dimers through interaction with their concave sides (Scott et al., 2006). Through gel filtration chromatography, light scattering and sedimentation equilibrium, opticin has also been 
shown to form dimers (Le Goff et al., 2003). These data suggest that the biologically active form of decorin, opticin and biglycan may be a dimer.

Nyctalopin has been predicted to be oriented with the $\mathrm{N}$-terminus in the extracellular space and anchored to the cell surface by a GPI moiety (human) or two transmembrane domains in rodents (Gregg et al., 2003). The core protein is predicted to have five $\mathrm{N}$-linked oligosaccharide or keratin sulfate glycosylation sites $(\mathrm{N}-\mathrm{X}-(\mathrm{S} / \mathrm{T}))$ (Bech-Hansen et al., 2000; O'Connor et al., 2005). In this chapter, I used a combination of yeast two-hybrid and in-vitro translation approaches to show that murine nyctalopin is oriented with the N-terminus in the extracellular space and that it is anchored to the membrane by a single transmembrane domain. I also determined that nyctalopin does not form homo-dimers.

\section{METHODS}

\section{Yeast Strains and Growth Media}

Yeast strains used in this study are NYM32 (MATa his3delta200 trp1-901 leu23,112 ade2 LYS2::(lexAop) 4 -HIS3 URA3::(lexAop) $)_{8}$-lacZ (lexAop) ${ }_{8}$-ADE2 GAL4)) and BY4741 (MATa; his $3 \Delta 1$; leu2 $\Delta 0$; met $15 \Delta 0$; ura3 $\Delta 0$ ) strains. The growth media used is synthetic media lacking leucine, tryptophan (SD/-Leu/-Trp), and leucine, tryptophan, histidine and adenine (SD/-Leu/-Trp/-His/-Ade) (Clontech, Moutain View, CA). All transformations were done using the lithium chloride method (Clontech, Moutain View, CA).

\section{Plasmid constructions for topology experiments}

Full length cDNA clones' representing bipolar cell genes were performed using retinal cDNA synthesized from adult $C 57 B 6 / 6 J$ mice retinal mRNA. Vectors were 
constructed using infusion cloning techniques (Clontech, Moutain View, CA). For membrane topology experiments, Eyfp-Cub was made by cloning an enhanced yellow fluorencence protein cNDA into the PstI site of the pCCW-SUC vector (Dualsystems, Grabenstrasse, Switzerland). Nyc-Cub was made by inserting the nyctalopin cDNA encoding amino acids 23-476 into the SfiI site of Eyfp-Cub vector fusing the C-terminus of nyctalopin to the C-terminus of ubiquitin $(\mathrm{Cub})$ and the synthetic transcription factor (LexAVP16). To generate Cub-Nyc, the signal sequence of invertase gene was cloned into the XbaI site of the pNCW vector (Dualsystems, Grabenstrasse, Switzerland). Nyctalopin cDNA encoding amino acids $23-476$ was cloned into the $P$ st $I$ site of pNCW (Dualsystems, Grabenstrasse, Switzerland). Grm6 -Cub was made by cloning a full length Grm6 cDNA without its signal sequence into the SfiI site of pCCW-SUC (Dualsystems, Grabenstrasse, Switzerland).

Fur4-NubI was made by digesting pAI-Alg5 (Dualsystems, Grabenstrasse, Switzerland) with SpeI and ClaI enzymes to remove the Alg5 gene and inserting the Fur4 gene by infusion cloning. Alg5-NubG was made by digesting pAI-Alg5 (Dualsystems, Grabenstrasse, Switzerland) with AgeI and XhoI which removes the N-terminus of ubiquitin (NubI) from the pAI-Alg5 vector. The forward primer used to PCR NubI has an Isoleucine (I) to glycine $(\mathrm{G})$ substitution at position 13 . The resulting PCR product was cloned into the AgeI and XhoI double digested vector. Fur4-NubG was made by cloning the Fur4 gene into the pDL2Nx prey vector (Dualsystems, Grabenstrasse, Switzerland). Nyctalopin homodimerization was assayed by cloning full length nyctalopin cDNA into the SfiI site of the pDL2Nx prey vector (Dualsystems, Grabenstrasse, Switzerland). This vector was named Nyc-NubG. As a positive control, cDNA encoding synaptopysin (Syp) 
was cloned into the SfiI site of the pCCW-Ste bait vector (Dualsystems, Grabenstrasse, Switzerland) and pDL2N-Ste prey vector (Dualsystems, Grabenstrasse, Switzerland) respectively. These vectors are called Syp-Cub and Syp-NubG, respectively.

For mutagenesis experiments, nyctalopin cDNA encoding amino acids 23-452 (lacks signal sequence and TM3) was cloned into the SfiI site of the Eyfp-Cub vector (Dualsystems, Grabenstrasse, Switzerland). This vector was called Nyc $\Delta \mathrm{TM} 3-\mathrm{Cub}$. A plasmid encoding a leucine (L) to arginine (R) mutation at position 463 was made by creating the mutation in one of the infusion primers. This primer was used to PCR just the TM domain of nyctalopin. The amplified TM domain was cloned into the NheI site of Nyc $\Delta$ TM3-Cub vector. This vector was named NycL463R-Cub vector.

\section{Constructs used in the in vitro transcription/translation experiments}

The SNycLuc vector was made by inserting full length nyctalopin cDNA into the BamH1 site of the T7 Luciferase Control vector (Promega, Madison, WI). This arrangement fused luciferase to the C-terminus of nyctalopin. For the SLucNyc vector, the luciferase gene was removed from the T7 Luciferase control vector (Promega, Madison, WI) by double digestion with BamHI and SacI restriction enzymes. The multiple cloning site (MCS) was amplified from the T7 Luciferase Control vector (Promega, Madison, WI) and infusion cloned back into the BamHI and SacI double digested T7 Luciferase Control vector (Promega, Madison, WI). This vector was named T7-Base vector. The T7-Base vector was linearized with BamHI and nyctalopin signal sequence was inserted to produce T7SSNYX. The T7SSNYX vector was linearized with BamHI and the luciferase gene was inserted by infusion cloning to produce T7SLUC vector. Finally, the T7SLUC vector was linearized with BamHI and nyctalopin (aa 23- 
476) was inserted by infusion cloning. This produced SLucNyc vector, which when expressed produces a protein that has the nyctalopin signal sequence fused to the luciferase that in turn is fused to the rest of nyctalopin. Whenever infusion cloning was performed, a BamHI site was destroyed at the N-terminus and a new one was created at the C-terminus.

\section{Colony Lift Assay}

This assay was used to examine the expression of $\beta$-galactosidase. A single colony was picked from a selection plate, streaked onto a new Synthetic dropout (SD) plate (SD/Leu/-Trp) and incubated for 2 to 3 days at $30^{\circ} \mathrm{C}$. A piece of circular Whatman $3 \mathrm{MM}$ filter paper was placed directly onto the agar plate in contact with the yeast colonies for 10 minutes to allow the yeast to bind to the filter paper. The filter paper then was carefully removed from the plate and transfered into liquid nitrogen for 2 minutes to lyse the yeast cells. The filter paper was then placed in a petri-dish with yeast side up and allowed to thaw for 5 minutes at room temperature. The filter paper was incubated in freshly prepared Agarose X-Gal mix (0.5g Agarose, $100 \mathrm{ml}$ of $1 \mathrm{xPBS}$ and $500 \mu \mathrm{l}$ of $20 \mathrm{mg} / \mathrm{ml} \mathrm{X-}$ gal) at room temperature for up to 8 hours or until a blue color forms.

\section{Immunohistochemistry}

Yeast were grown to mid- $\log \left(\mathrm{OD}_{600}=0.5-0.75\right)$ and fixed in $2 \mathrm{X}$ fixing solution $(2 \%$ glucose, $0.04 \mathrm{M}$ EGTA, 7.4\% formaldehyde, 1x PBS) for 1 hour. The cells were recovered by centrifuging at $3000 \mathrm{xg}$ for 5 minutes and washed two times in PS-Buffer $(0.1 \mathrm{M} \mathrm{NaPO} 3 \mathrm{pH} 6.6,1.2 \mathrm{M}$ sorbitol). To make spheroplasts, cells were incubated for 15 minutes in PS-buffer plus $10 \mu 1$ of $\beta \mathrm{ME}, 10 \mu \mathrm{l}$ of zymolyase $(50 \mathrm{u} / \mathrm{ml})$ at $37^{\circ} \mathrm{C}$ followed by two washes in PS-buffer. $50 \mu 1$ of cells were added to polylysine coated glass microscope 
slides, incubated at room temperature for 15 minutes and washed in PS-buffer three times. The cells were permeabilized in P-buffer $(0.01 \mathrm{~g} / \mathrm{ml}$ of BSA, $0.5 \%$ SDS, $1 \times$ PBS $)$ for 15 minutes, washed ten times in PBS/BSA-Buffer $(0.01 \mathrm{~g} / \mathrm{ml}$ of BSA, 1xPBS) and then blocked for 1 hour in PBS/BSA buffer. At the end of blocking, cells were washed five times in PBS/BSA buffer by applying the buffer and aspirating. Primary GFP conjugated Alexa 488 antibody (Lot \#: A2131, Invitrogen, Carlsbad, CA) at a 1:1000 dilution was added to the cells in PBS/BSA buffer and incubated in a moist chamber overnight. Cells were then washed ten times with PBS/BSA-Buffer by applying the buffer on to the slides and aspirating. Finally cells were washed three times in PBS and allowed to air dry at room temperature before covering them with a coverslip. Images were taken on an Olympus FV300 Confocal Microscope using a 60x objective and images processed using Flowview software.

\section{In vitro translation and the protection assays}

In vitro transcription/translation was done according to the manufacturer's protocol (Promega, Madison, WI, cataloque\# TM035). Plasmid DNA ( $2 \mu \mathrm{g})$ was added to each translation reaction. To maximize the translation of membrane proteins, canine microsomal membranes (Promega, Madison, WI, catalogue\# Y4041) were added to some samples. After 90 minutes incubation at $30^{\circ} \mathrm{C}, 2 \mu 1$ of the translation mix was added to $13 \mu \mathrm{l}$ of SDS sample buffer and heated at $70^{\circ} \mathrm{C}$ for 10 minutes. Samples were resolved by PAGE on 4-12\% NOVEX gradient gels (Invitrogen, Calsbad, CA).

To determine orientation of proteins in the membrane, $10 \mu \mathrm{l}$ of translation product was incubated with or without $0.1 \mu \mathrm{g} / \mu \mathrm{l}$ of proteinase K. Samples were incubated on ice for 5 , $10,15,20$ minutes with or without the addition of Chaps $(0.5 \%)$. For experiments where 
the canine microsomal membranes were dissolved, Chaps $(0.5 \%)$ was added to the translation mix, incubated for 15 minutes, and centrifuged (14000g) for 25 minutes. The supernatant was removed and thrombin cleavage assay mix was added to the samples. Thrombin digestions contained in $50 \mu \mathrm{l} ; 1 \mathrm{x}$ thrombin cleavage buffer, $10 \mu \mathrm{g}$ of solubilized or unsolubilized SLucNyc protein, and thrombin (50U) in a final volume of $50 \mu \mathrm{l}$. Samples were incubated at $20^{\circ} \mathrm{C}$ for 16 hours. $20 \mu \mathrm{l}$ of the digested samples was added to $20 \mu 1$ of SDS buffer, heated at $70^{\circ} \mathrm{C}$ and resolved by PAGE on $4-12 \%$ NOVEX gradient gels (Invitrogen, Calsbad, CA).

\section{RESULTS}

\section{Topology of murine nyctalopin}

Nyctalopin was predicted to be bound to the membrane by a GPI anchor in human and have two transmembrane domains in rodents (Bech Henson et al., 2000; Gregg et al., 2003). Expression in cultured cells provided some experimental support for these predictions, although the mechanism and orientation of murine nyctalopin was less certain (Zeitz et al., 2003; O'Conner et al 2005). I used computer predictions and direct experimental testing to determine the membrane topology and the mechanism of membrane anchoring of nyctalopin.

I analyzed the primary sequence of murine nyctalopin using seven different topology prediction programs, each of which uses slightly different prediction algorithms. For all the programs I used default parameters set by each program. Table 1 summarizes my results from these analyses. It can be seen that there is no consensus with respect to the number and/or even the presence of transmembrane domains in murine nyctalopin 


\section{Table 1. Predicted membrane topology of nyctalopin}

\begin{tabular}{|c|c|c|c|c|c|c|}
\hline Proglans & \# TM(s) & C-telminus & $\mathrm{N}$-terminus & pTMIl & pTM2 & pTM 3 \\
\hline HMMMTOP 20 & 3 & OUT & IN & $260-278$ & $309-328$ & $452-473$ \\
\hline TMPred & 2 & INIOUT & חN/OUT & None & $309-329$ & $455-471$ \\
\hline TopPred2 & 2 & NVOUT & IN/OUT & None & $309-328$ & $455-471$ \\
\hline SOSUI-Mp 1 & 1 & $\mathbb{N}$ & OUT & None & None & $452-473(1.0)$ \\
\hline ConPred II & 1 & $\mathbb{N}$ & OUT & None & None & $455-473$ \\
\hline TMLTMM2 0 & $0 / 2$ & NNOUT & TIVIOUT & None & $309-329(0.6)$ & $455-473(0.9)$ \\
\hline DAS & 0 & NONE & OUT & None & None & None \\
\hline
\end{tabular}

pTM denote the predicted transmembrane domain. Numbers under the pTM correspond to amino acid residue of nyctalopin. \# denotes the number of predicted transmembrane domainS. IN means the N-terminus (N-Ter) of nyctalopin is in the cytoplasm and the Cterminus is in the extracellular space. The numbers in bracket denote the probability of the presence of a transmembrane domain. 
Figure 12. Schematic drawing of the different predicted membrane topologies of nyctalopin. A. Nyctalopin protein with the predicted transmembrane domain marked. B. The different ways nyctalopin is oriented in the membrane. $\mathbf{i}$ shows that nyctalopin has three transmembrane domains with the $\mathrm{N}$-terminus in the cytoplasm. ii shows that nyctalopin has two transmembrane domain and both can either be in the cytoplasm or in the extracellular space. iii shows that nyctalopin has a single transmembrane domain with the N-terminus in the extracellular space. iv shows that nyctalopin has no transmembrane domain. 
A

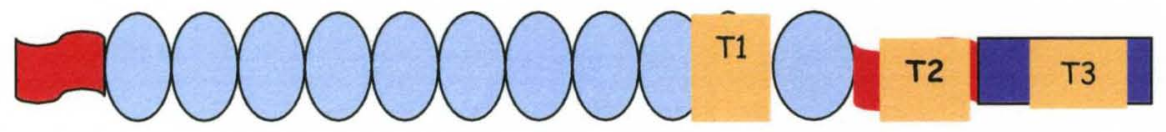

B

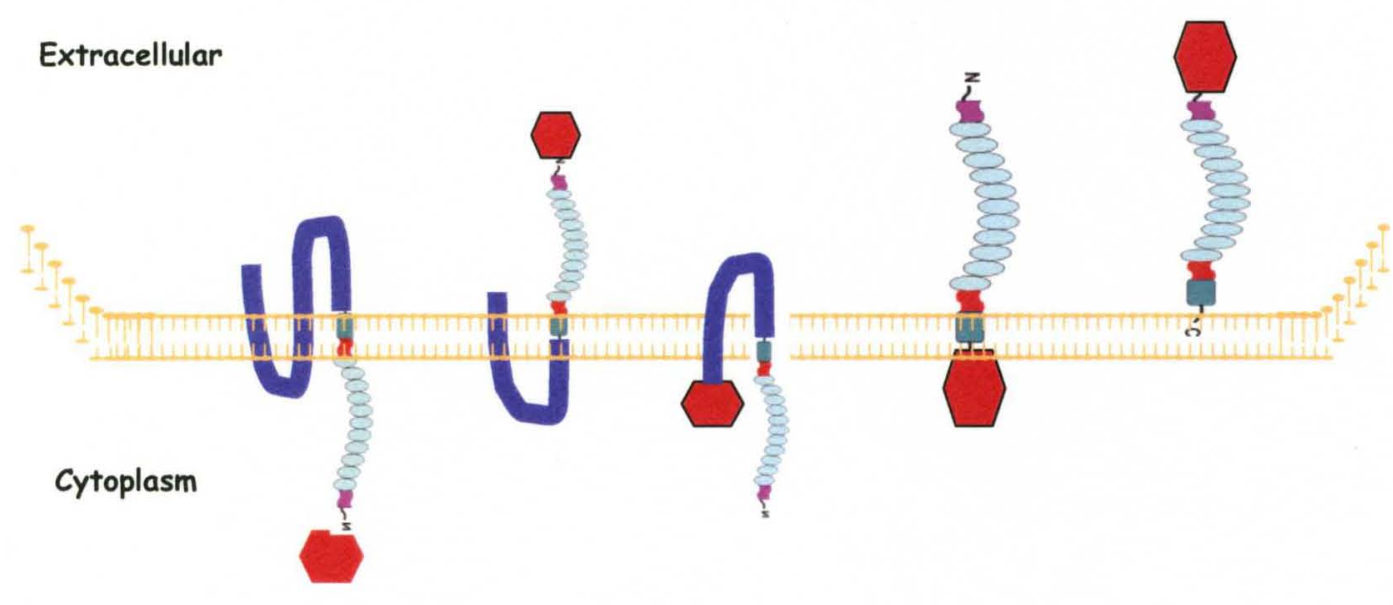

I.

II.

III.

IV 
(Table 1). Five of the seven programs, SOSUI (Hirokawa, 1998), TMPred (Hofmann and Stoffel, 1993), ConPred II (Arai et al., 2004), TopPred 2 (Carlos et al., 1994), HMMTOP2.0 (Tusnady et al., 1998), predict a transmembrane domain at position 455473 (TM3). Three of the programs (TMPred, TopPred2, HMMTOP2.0) predict a transmembrane domain at position 309-328 (TM2) and one program (HMMTOP2.0) predicts a single transmembrane domain at position 260-278 (TM1). Finally DASTMfilter (Cserzo et al., 2002) fails to identify any transmembrane domains.

One of the most commonly used prediction programs is TMHMM2.0 (Krogh et al., 2001) and it predicts nyctalopin either lacks or has two putative transmembrane domains. ConPredII aligns the transmembrane domain(s) predicted by each of these programs and reports the consensus TM domain and predicts only TM3. From these analyses, there may be as many as three transmembrane domains in murine nyctalopin (Figure 12). Figure 12 is schematic drawing of the different topologies of nyctalopin in the membrane as predicted by the different programs. The most support is for TM3 (452472), then TM2 (309-328), and there is a very weak support for TM1 (260-278) being a real transmembrane domain. In the sections below I use yeast two-hybrid and in vitro translation experiments to examine these predictions.

\section{Nyctalopin's leucine rich repeat (LRR) domain is oriented into the extracellular matrix}

To examine the membrane topology of nyctalopin, I used a membrane split ubiquitin yeast two hybrid system (MYTH). The MYTH system is based on the ability of the $\mathrm{N}$ - and the $\mathrm{C}$-terminal domains of ubiquitin to interact even when they are two separate peptides (Johnsson et al., 1994). When each (Cub or Nub) is fused to another 
protein, the two ubiqutin domains can interact and the reconstituted ubiquitin molecule is functional and can be recognized by ubiquitin proteases for cleavage (Johnsson et al., 1994; Stagljar et al., 1998; Stagljar et al., 2003). In the MYTH system, the bait (a transmembrane bound protein) is expressed as a fusion protein with the C-terminal half of ubiquitin ( $\mathrm{Cub}$ ) and a synthetic transcription factor (LexA-VP16). The prey is expressed as a fusion protein with either the $\mathrm{N}$-terminal half of ubiquitin (NubI) or its mutated form (NubG) (Figure 13). In NubG, the isoleucine at position 13 in the $\mathrm{N}$ terminus of ubiquitin is substituted for glycine. This substitution prevents the reconstitution of the two ubiquitin halves and makes the interaction of the two halves dependent on the interaction of the fusion test proteins (Johnsson et al., 1994; Stagljar et al., 1998).

To assay for the topology nyctalopin, I used fusion proteins containing Cub and NubI (Figure 13A). Cub and NubI can interact without interaction of the bait and prey protein, provided the $\mathrm{Cub}$ and $\mathrm{NubI}$ are localized in the cytoplasm. To assay for interaction of bait and prey proteins, Cub and NubG fusion proteins are used (Figure 13B). Cub does not interact with NubG unless the bait and prey proteins interact and bring the two close enough to reconstitute the ubiquitin molecule (Stagljar et al., 1998; Stagljar et al., 2003). The reconstituted ubiquitin molecule is recognized by the ubiquitin proteases in the cytoplasm, which cleave the synthetic transcription factor (LexA-VP16). LexA-VP16 then translocates into the nucleus and activates reporter genes.

To evaluate cellular localization of nyctalopin, I created an EYFP tagged nyctalopin (Nyc-Cub) that could be expressed in yeast (Figure 14A). As a control, I used the same vector to express EYFP fused to Cub (EYFP-Cub) (Figure 14A). These 
plasmids were introduced into yeast strain BY4741 and the sub-cellular localization of each determined by confocal microscope (Figure 14B). These images show that Nyc-Cub is localized to the plasma membrane. In contrast, EYFP-Cub is present in the cytoplasm and is excluded from the vacuoles (Figure 14B). To confirm that nyctalopin was localized to the membrane, I fractionated yeast lysates into membrane and cytosolic fractions. A known ER membrane bound yeast protein (Alg5-Cub) was used as a positive control. Sub-cellular fractions of cells containing all three constructs were analyzed by western blot using an antibody to LexA. Figure 14C shows that both Nyc-Cub and Alg5-Cub fusion proteins are localized to the membrane fraction.

After confirming that nyctalopin was properly targeted to the membrane in yeast cells, I explored its orientation using the MYTH system. As a positive control, I attached Cub-VP16LexA to the C-terminus of the Grm6 receptor, which is known to be cytoplasmic (Romano et al., 1996). To determine the orientation of nyctalopin, I attached the Cub-VP16LexA-domain to either the C-terminus (Nyc-Cub) or the N-terminus (CubNyc) of nyctalopin (Figure 15A). To generate a prey protein with known sub-cellular location I attached NubI to the C-terminus of the yeast plasma membrane protein; uracil permease (Fur4). Fur4 is known to orient in the plasma membrane with both $\mathrm{C}$ - and Ntermini in the cytoplasm (Garnier et al., 1996; Heesen et al., 1994). Co-transformation of Grm6-Cub and Fur4-NubI into NMY32 yeast results in growth on selective plates as well as a positive X-gal assay (Fig 15B, row 1, panel 2\&3). This shows that Grm6 is oriented in yeast as in bipolar cells. To test nyctalopin orientation either Nyc-Cub or Cub-Nyc was co-transfected with Fur4-NubI. Successful co-transformation was determined by 
Figure 13. Schematic representation of membrane split ubiquitin yeast two hybrid system (MYTH). A. Co-expression of Cub and NubI result in their interaction. On interaction of Cub and NubI, ubiquitin proteases in the cytoplasm cleave the synthetic transcription factor (LexAVP16) which translocates into the nucleus and activates the transcription of target genes. In this system the target genes are histidine, adenine and $\beta$ galactosidase. B. Co-expression of Cub and NubG fusion proteins. The interaction of NubG with Cub is dependent on the interaction of the two fusion proteins. The interaction brings the $\mathrm{Cub}$ and NubG (mutated form of NubI) together to form a split ubiquitin molecule. The split ubiquitin molecule is recognized by ubiquitin proteases in the cytoplasm that cleave the protein releasing the synthetic transcription factor (LexAVP16). The transcription factor translocates into the nucleus and activates the transcription of reporter genes, histidine, adenine and $\beta$-galactosidase. 
A.

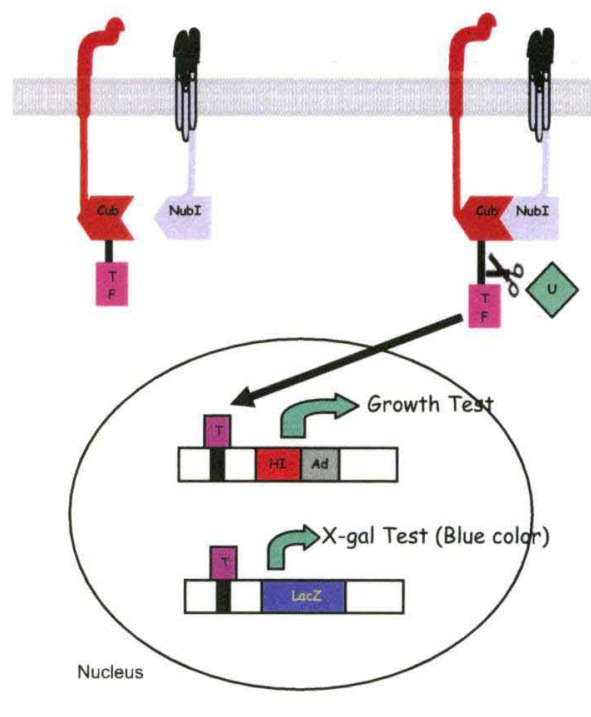

B.

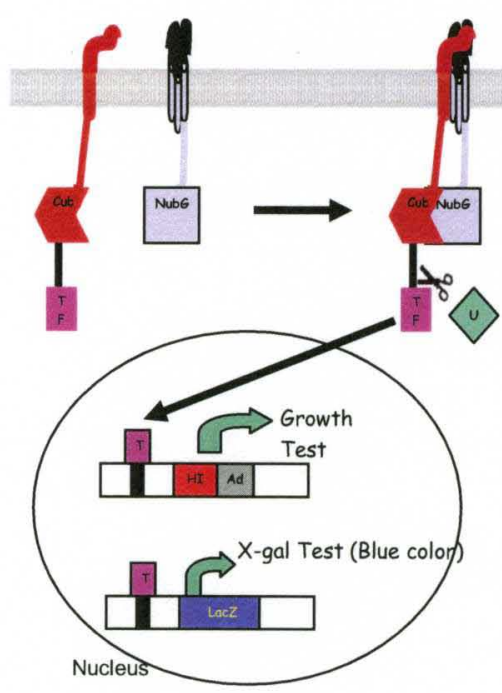


selection on SD-LW plates (Figure 15B, Left column). Interactions were determined by growth on SD-LWHA plates and X-gal assay (Figure 15B). These data show that growth as well as $\beta$-galactosidase expression is negative for Cub-Nyc. In contrast, both growth and $\beta$-galactosidase expression is positive for $\mathrm{Nyc}-\mathrm{Cub}$ indicating that the $\mathrm{C}$-terminus of nyctalopin is intracellular. That only Nyc-Cub shows interaction indicates that nyctalopin cannot have an even number of transmembrane domains (Figure 16ii). The negative results for Cub-Nyc also showed that nyctalopin $\mathrm{N}$-terminus is not in the cytoplasm (Figure16i and 16iv).

\section{Murine nyctalopin has a single transmembrane domain}

The previous experiments show that nyctalopin is a membrane bound protein with its C-terminus in the cytoplasm. To map the transmembrane domain of nyctalopin, I made deletion or mutation constructs of nyctalopin and tested their topology using the MYTH system (Figure 17A). First, I tested whether nyctalopin is inserted into the membrane when the entire predicted transmembrane domain is removed (Nyc $\Delta T M 3$ Cub) (Figure 17A). Also, I created a leucine to arginine mutation at positon 463 (NycL463R-Cub), which is in the predicted transmembrane domain (TM3) of nyctalopin. Substituting the hydrophilic arginine residue should shorten the hydrophobic stretch and interferes with membrane insertion. To confirm that the mutation L463R would disrupt the TM3, I analyzed the mutant protein with TMHMM2.0 and as expected the analysis interfere with membrane insertion. To confirm that the mutation L463R would disrupt the TM3, I analyzed the mutant protein with TMHMM2.0 and as expected the analysis indicated that TM3 would be disrupted $(\sim 0.2$ probability of TM). If TM3 is the only transmembrane domain, the deleted or mutant nyctalopin protein should be secreted into 
Figure 14. Nyctalopin is localized to the membrane in yeast. A. Vector constructs built to determine the localization of nyctalopin in yeast. Nyc-Cub $(109.4 \mathrm{kDa})$ is a fusion protein with the yeast invertase signal sequence (SUC) (20aa), Eyfp (217aa), nyctalopin (453aa) and the Cub-LexAVP16 (350aa) artificial transcription factor. Alg5-Cub (77.5 $\mathrm{kDa}$ ) is a yeast ER membrane bound protein fused to Cub-LexAVP16 and is used as positive control for western blotting. Untransfected yeast cells are used as negative control for western blotting. Eyfp-Cub is Eyfp fused to Cub-LexAVP16 and used as a control for confocal imaging. B. Confocal image of yeast strain BY4741 expressing Eyfp-Cub or Nyc-Cub. Images show that Nyc-Cub is localized to the plasma membrane and Eyfp-Cub is in the cytoplasm. C. Western blot of membrane (M) and cytosolic (C) fractions of yeast strain NMY32 transfected with Nyc-Cub, negative control (untransfected NMY32) and Alg5-Cub. These data indicate nyctalopin is membrane bound. 
A

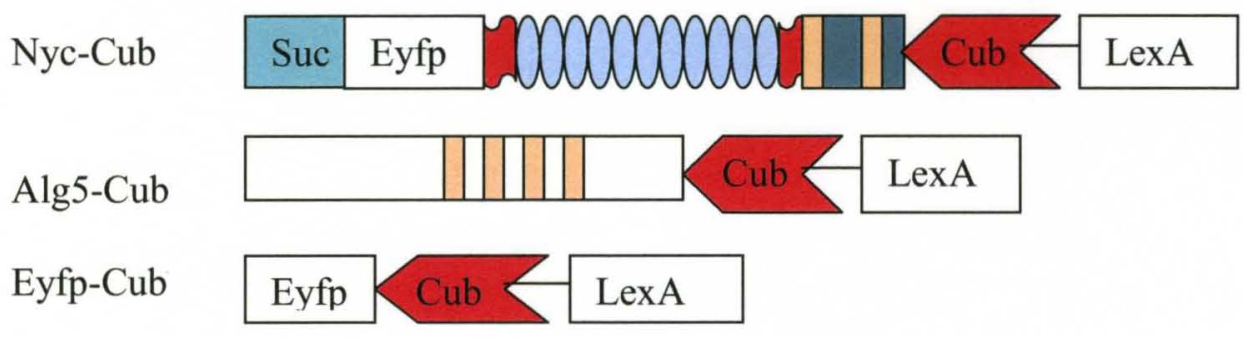

B.

Eyfp-Cub

Nyc-Cub
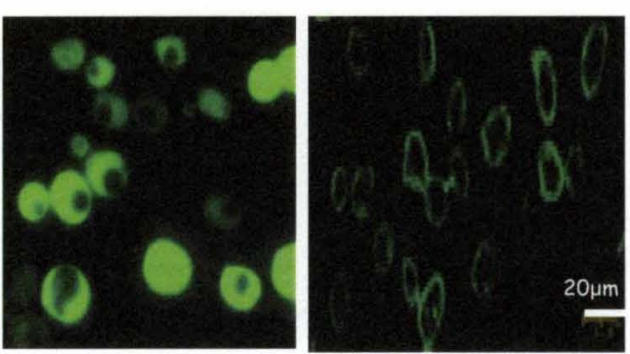

C. Alg5-Cub Control Nyc-Cub

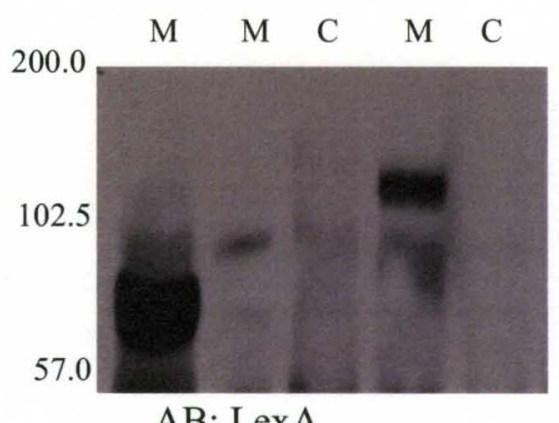

AB: LexA 


\section{Figure 15. The $\mathbf{N}$-terminus of nyctalopin is extracellular.}

A. Vector constructs used to determine the orientation of nyctalopin in the membrane. Bait constructs use the yeast invertase (SUC) signal sequence. Cub-Nyc has CubLexAVP16 inserted between the SUC signal sequence and nyctalopin.

B. Membrane yeast two hybrid analysis of nyctalopin orientation in the membrane. Plasmid incorporation (column 1) is confirmed by growth on SD/-LW plates. When NubI and CubLexAVP16 are localized in the cytoplasm interaction will occur supporting growth on SD/-LWHA media and expression of $\beta$-galactosidase. Growth of Nyc-Cub and Fur4-NubI on selective SD-LWHA media indicates the C-terminus of nyctalopin is localized in the cytoplasm. No growth of Cub-Nyc and Fur4-NubI on selective SDLWHA indicates that the N-terminus of nyctalopin is in the extracellular space. Grm6 Cub is used as a positive control. 
A.

\section{Bait:}

Nyc-Cub

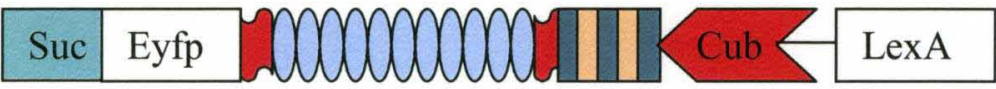

Cub-Nyc

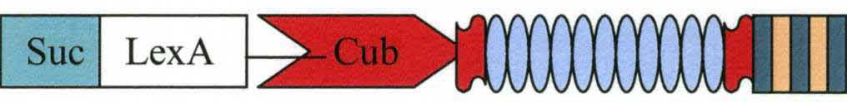

GRM6-Cub

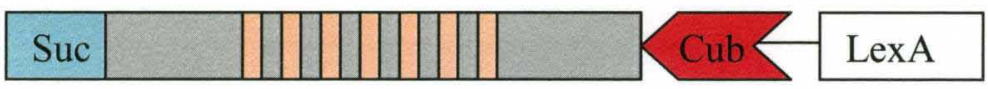

Prey:

Localization:

Fur4-NubI

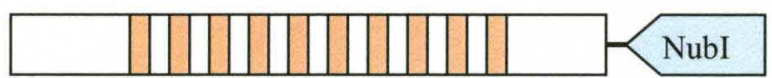

PM

B.

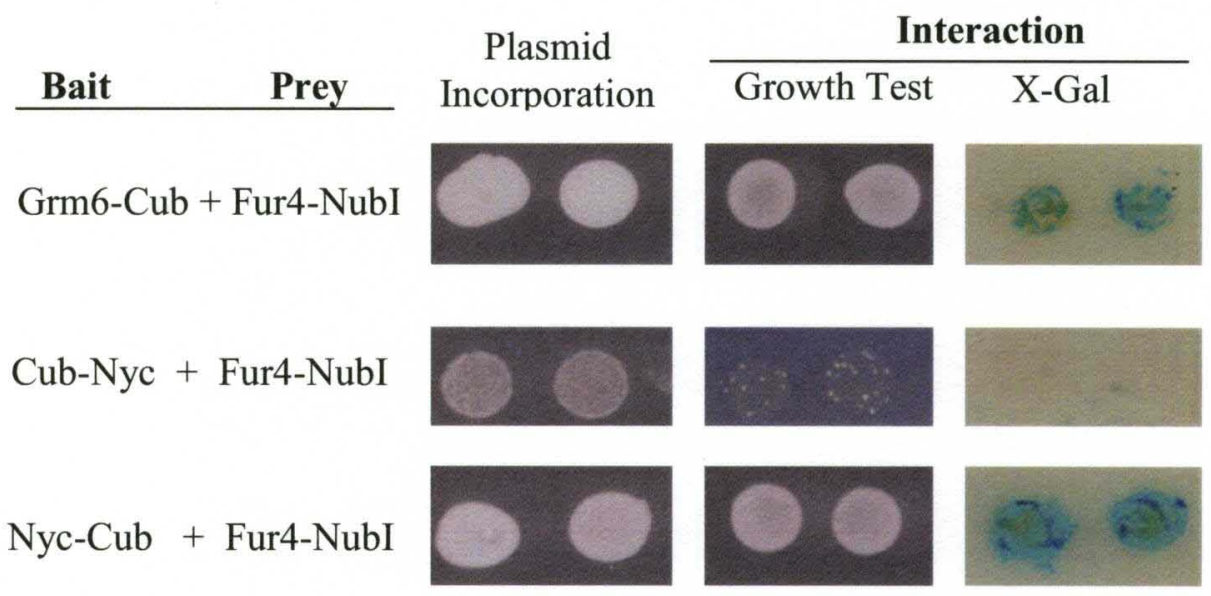


Figure 16. Schematic of the different predicted membrane topologies of nyctalopin. A. The different orientation of nyctalopin as predicted by transmembrane prediction programs. Experimental data eliminated all possible topologies except one. Nyctalopin is orientated with the entire LRR domain in the extracellular space indicated here by the circled figure. B. It is still not clear which transmembrane domain $(\operatorname{Tm} 2$ or $\operatorname{Tm} 3)$ is used to anchor nyctalopin to the membrane. 
A.

\section{Extracellular}

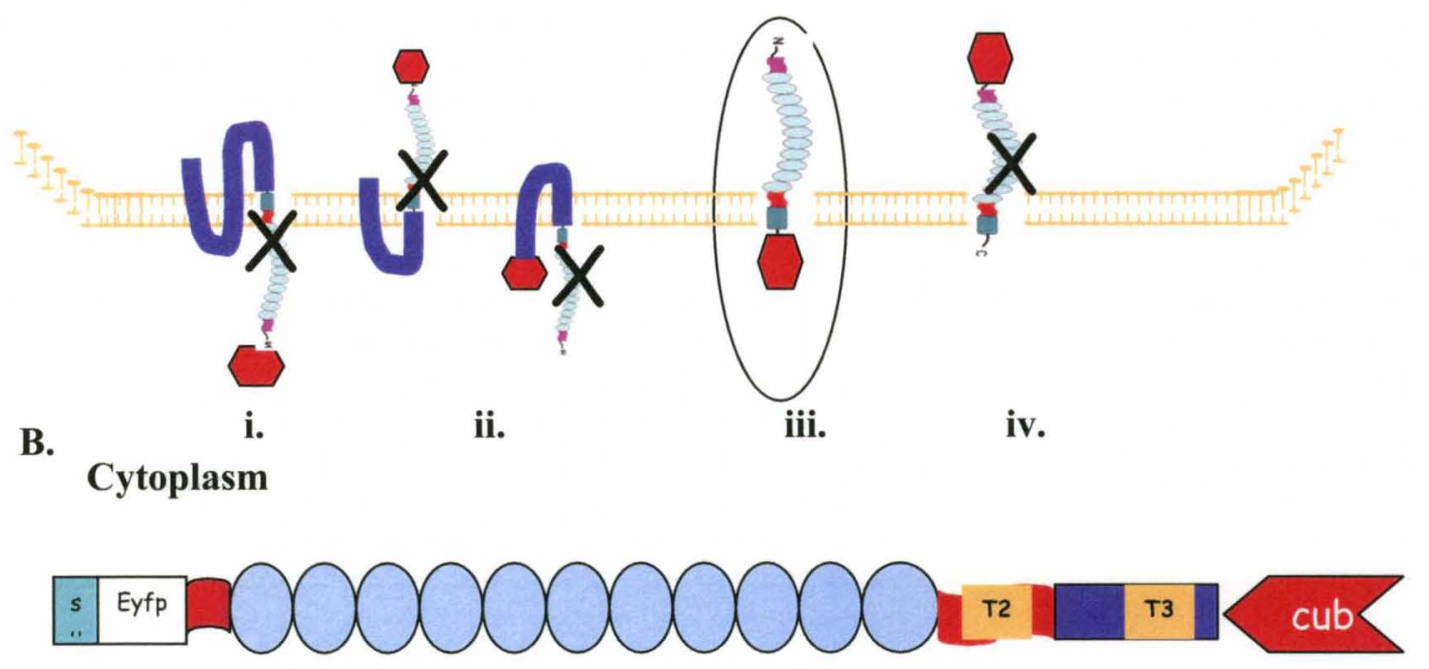


the lumen of the ER (NycATM3-Cub or NycL463R-Cub). This should prevent interaction of the attached Cub with NubI expressed in the cytoplasm. The Nyc-Cub and the two mutant constructs were each co-transfected with three different prey vectors. Fur4-NubI was used to test for membrane insertion and orientation. Fur4-NubG was used to test for specificity of interaction between Cub and NubI, and a control vector expressing only NubG was used to test for self- activation (Figure 17A, Prey). The first three columns of Figure 17Bi shows that all plasmids are incorporated into the yeast and express the selectable markers. Figures $13 \mathrm{Bii}$ and $13 \mathrm{Biii}$ shows that the only combination of bait and prey vectors that supported robust growth and expression of $\beta$-galactosidase was Nyc-Cub/Fur4-NubI. Neither of the mutants with altered transmembrane domains (Nyc $\Delta$ TM3-Cub and NyxL463R-Cub) supported growth or expression of $\beta$-galactosidase as measured by the $\mathrm{X}$-gal assay (Figure $17 \mathrm{Bii} \&$ iii). The failure of growth and $\beta$ galactosidase expression when Nyc-Cub and Fur4-NubG is co-expressed indicates that the Nyc-Cub/Fur4-NubI interaction is not because of some non-specific interaction of nyctalopin and Fur4 proteins.

The data above assume that the deletion and mutation constructs I created were expressed in yeast cells. This was confirmed by analyzing yeast extracts by western blots using antibodies to LexA. These proteins were expressed in all cases (Figure $17 \mathrm{C} \mathrm{i).} \mathrm{The}$ other possibility for this negative result could be Nyc $\Delta T M 3-C u b$ and NyxL463R-Cub fusions proteins are expressed at levels undetectable by both growth and Xgal assays. Figure 17C ii show that Nyc-Cub was expressed at a lower level than both Alg-Cub and Nyc $\Delta$ TM3-Cub. Alg5-Cub is positive for growth and Xgal while Nyc $\Delta T M 3-C u b$ is 
negative for both growth and Xgal, suggesting that the negative result is not due to low levels of expression of these Nyc $\Delta \mathrm{TM} 3-\mathrm{Cub}$ and NyxL463R-Cub fusion proteins.

The failure of the combination of $\mathrm{Nyc}-\mathrm{Cub} / \mathrm{NubG}$ to grow or express $\beta$ galactosidase suggests that murine nyctalopin is not GPI anchored. If it was, the carboxy Cub-VP16LexA would have been cleaved and this alone would have supported growth and $\beta$-galactosidase expression.

\section{Murine nyctalopin does not homodimerize in yeast}

Several members of the SLRP family including decorin, opticin, and biglycan have been shown to dimerize through their LRR domains (Scott et al 2003, Le Goff 2003, Scott et al 2006). To determine if nyctalopin could dimerize in yeast, I cloned full length nyctalopin with its signal sequence replaced by S.cerevisiae invertase signal sequence (SUC) into the prey vector pDL2N-SUC vector (Dualsystems). This fuses NubG to the C-terminal of nyctalopin (Nyc-NubG) (Figure 18A). I used synaptophysin-Cub (SypCub) and synaptophysin-NubG (Syp-NubG), which have been shown to form dimers using the MYTH system (Felkl and Leube, 2007) as a positive control. The Alg5-Cub and Alg5-NubG combination was used as negative control. Interactions between bait and prey vectors were tested by growth on SD-LWHA plates as well as the X-gal assay. The synaptopysin bait/prey combination showed both growth and expression of $\beta$ galactosidase as expected. However, neither the Alg5 nor the nyctalopin bait prey combinations showed either growth or expression of $\beta$-galactosidase (Figure 18B). These data provide evidence that nyctalopin does not form dimers in yeast. 
Figure 17: Genetic analysis shows murine nyctalopin has a single transmembrane domain. A. Deletion/mutation constructs used to map the transmembrane domain of nyctalopin. Nyc $\Delta \mathrm{TM}-\mathrm{Cub}$ has amino acid 455-476 (TM3) deleted from nyctalopin. NycL463R-Cub has a base substitution that creates a leucine to arginine substitution at position 463 of nyctalopin. Bi. Shows that all bait and prey plasmids were presence in the NMY32 yeast strain and supported growth on the selective SD/-LW plates. Bii. Growth test for interaction of bait and prey fusion protein when the transformants are grown on SD/-LWHA plates. Biii. An X-gal assay for expression of $\beta$-galactosidase confirming the interaction ii. These results support the conclusion that only Nyc-Cub/Fur4-NubI interact and that the transmembrane domain of nyctalopin requires localization to the cytoplasm C. Western blot showing truncated transmembrane and L463R mutants are expressed in yeast. 
A.

\section{Bait:}

Nyc-Cub

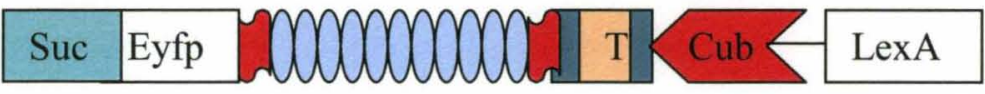

Nyc $\Delta \mathrm{TM}$

Suc Eyfp Allomoma $\mathrm{Cub} \leqslant$ LexA

NycL463R

Suc Eyfp Allomom $\mathrm{Cub}<\mathrm{LexA}$

\section{Prey:}

Fur4-NubI

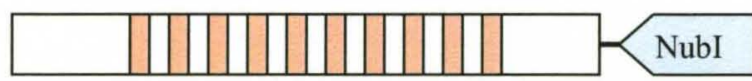

Fur4-NubG

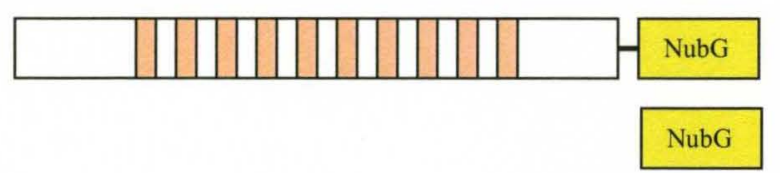

B.

i.

ii.

iii.

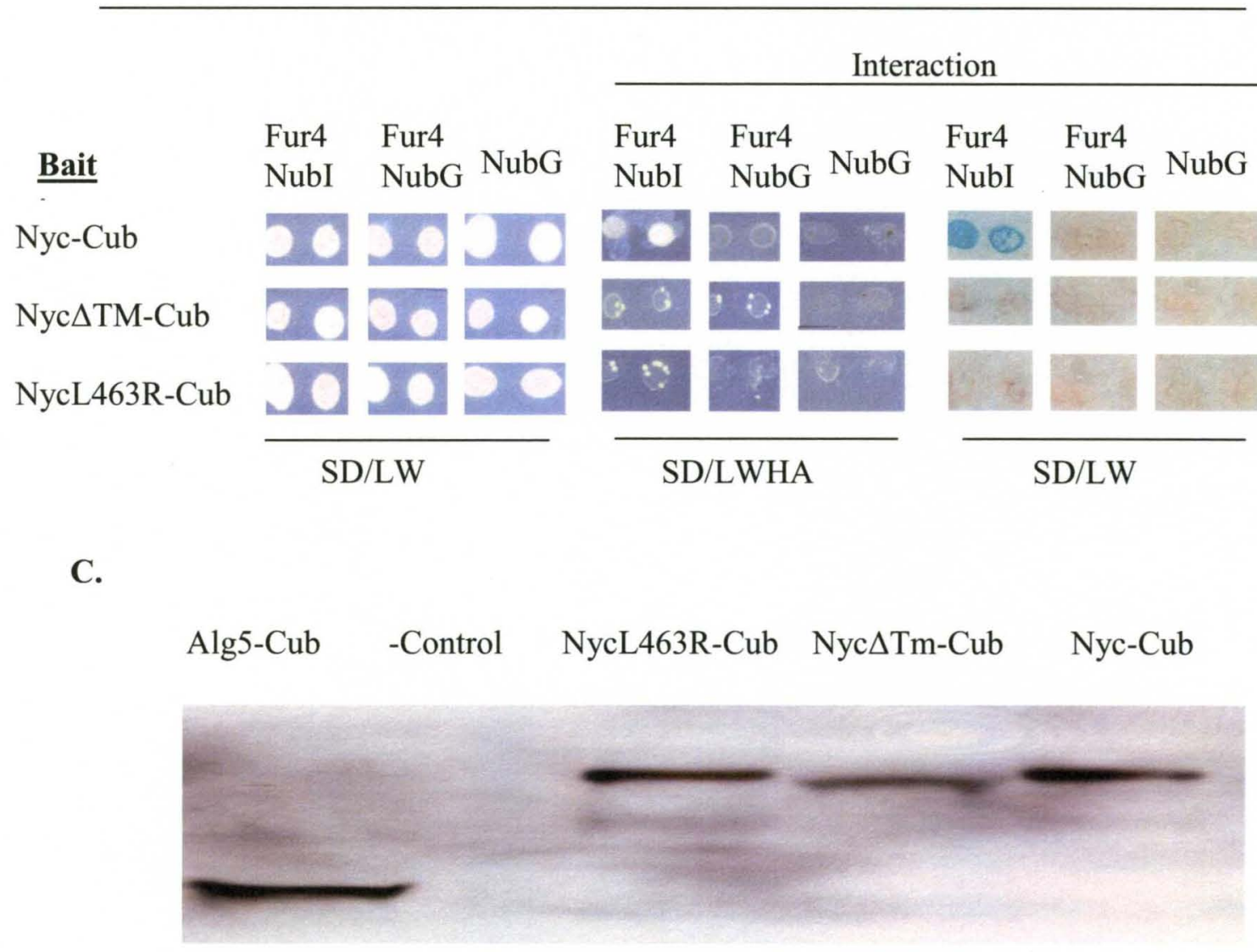

AB: Lex A 


\section{The LRR domain of nyctalopin is extracellular}

One of the limitations of the topology experiments in yeast is the fact that to obtain optimal expression of murine proteins, I had to replace the nyctalopin signal sequence with the $S$. cerevisiae invertase signal (SUC) sequence. This could potentially alter the topology of nyctalopin. To provide additional support for the proposed topology I used an in vitro transcription/translation system coupled with an ER rich canine microsomal membrane $(\mathrm{CMM})$ preparation. Detection of the translated proteins in this system was by incorporation of biotinylated lysine-tRNA (transcend tRNA), which is incorporated by the addition of precharged epsilon-labeled tRNA. This allows the use of streptavidin conjugated horseradish peroxidase (Strep-HRP) or streptavidin conjugated alkaline phosphatase (Strep-AP) for detection of newly synthesized protein on western blots. Nyctalopin only contains 2 lysines, therefore luciferase, which contains 40 lysines, was inserted after the nyctalopin signal sequence (SS) to increase detection sensitivity (SLucNyc) (Figure 16A). This should not disrupt function because insertion of EYFP at the same location generated a fully functional fusion protein (Gregg et al., 2007). A second vector with luciferase fused to the C-terminus of nyctalopin (SNycLuc), also was constructed (Figure 19A). A plasmid containing only luciferase was used as a positive control. First, I analyzed if nyctalopin is co-translationally processed by translating nyctalopin in the presence or absence of canine microsomal membrane (CMM). Nyctalopin is predicted to contain a signal sequence. This conclusion was supported by the observation that to obtain robust translation, addition of microsomal membranes, and incubation for indicated time period was needed. If the N-terminus is in the lumen of the microsomes as predicted, it should be protected from proteinase $\mathrm{K}$ 
Figure 18. Nyctalopin does not form homo-dimers in yeast. A. Bait and prey vectors used in the dimerization experiment. Nyc-Cub expressed a nyctalopin fusion protein with Eyfp fused to the N-terminus and the C-terminus of ubiquitin (Cub) plus the artificial transcription factor (LexAVP16) fused to the C-terminus. SUC is the yeast invertase signal sequence. Alg5, Asparagine-linked glycosylation 5, is a yeast ER membrane bound protein with both $\mathrm{C}$-terminus and $\mathrm{N}$-terminus in the cytoplasm. Syp-Cub is a bait vector with synaptophysin and is use as positive control. The prey vector is similar to the bait vectors except the CubLexAVP16 is replaced by NubG. B. Growth indicating incorporation of both bait and prey plasmids into yeast. The interaction was determined by selection on $\mathrm{SD} /$-LWHA plates and expression of $\beta$-galactosidase indicating that nyctalopin does not form dimers. 
A.

Bait:

Nyc-Cub

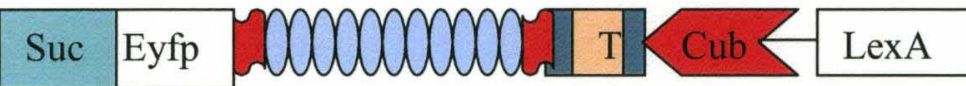

Alg5-Cub

Syp-Cub

Prey:

Nyc-NubG

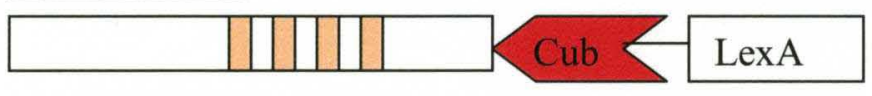

Alg5-NubG

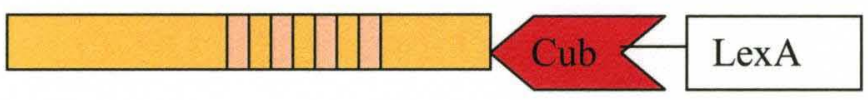

Syp-NubG
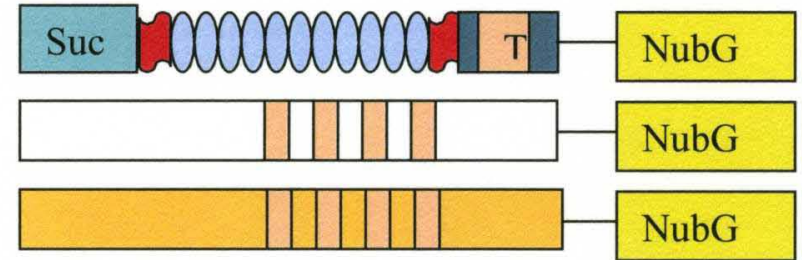

B.

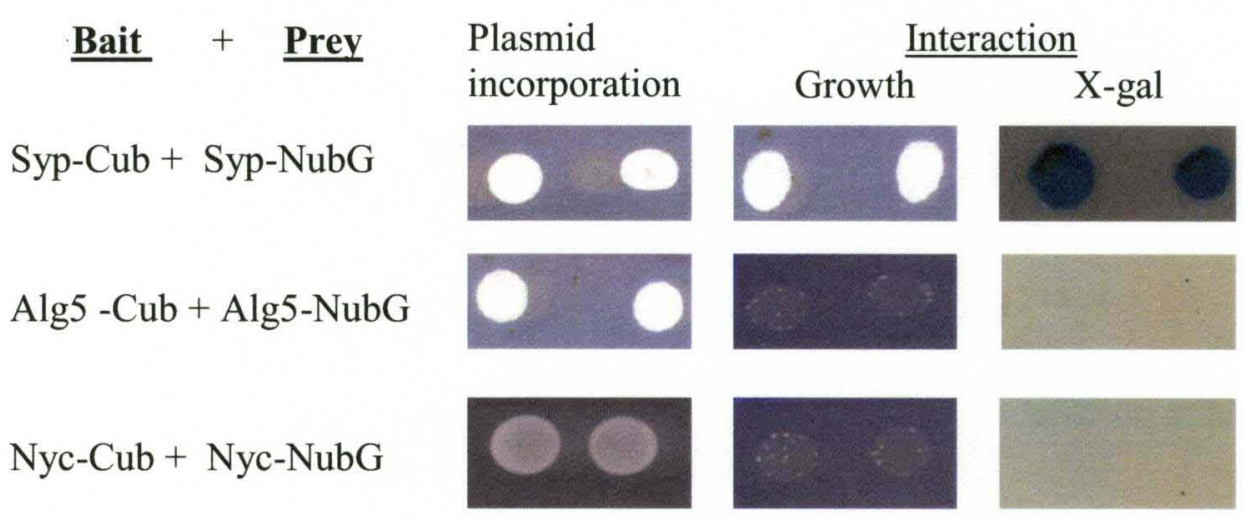


Figure 19. The N-terminus of nyctalopin is in the lumen of the ER. A. Vector constructs used to determine the orientation of nyctalopin in the membrane. SLucNyc $(113 \mathrm{kDa})$ has luciferase inserted after the nyctalopin signal sequence (SS). SS is nyctalopin's own signal sequence. SNycLuc has luciferase attached to C-terminus of full length nyctalopin. B. Western blot showing that without canine microsomal membrane $(\mathrm{CMM})$, nyctalopin is not expressed. These data indicate co-translational processing and membrane insertion of nyctalopin in the ER. C. A proteinase $\mathrm{K}$ digestion with or without chaps for both SLucNyx and SNycLuc. The first four Lanes indicate the expression of full length nyctalopin without proteinase $\mathrm{K}$ digestion. The next four lanes indicate proteinase $\mathrm{K}$ digestion without chaps. These data indicate that SLucNyc is protected from proteinase $\mathrm{K}$ digestion while SNycLuc is not. With the addition of Chaps (last four lanes), which disrupts the membrane, this protection is lost for both constructs suggesting that the $\mathrm{C}$-terminus of nyctalopin is in the cytoplasm and the $\mathrm{N}$-terminus is extracellular. 
A.

SLucNyc

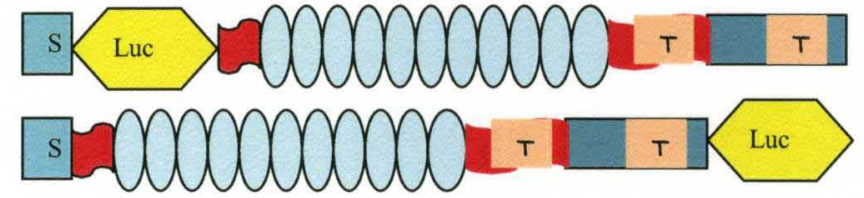

B.

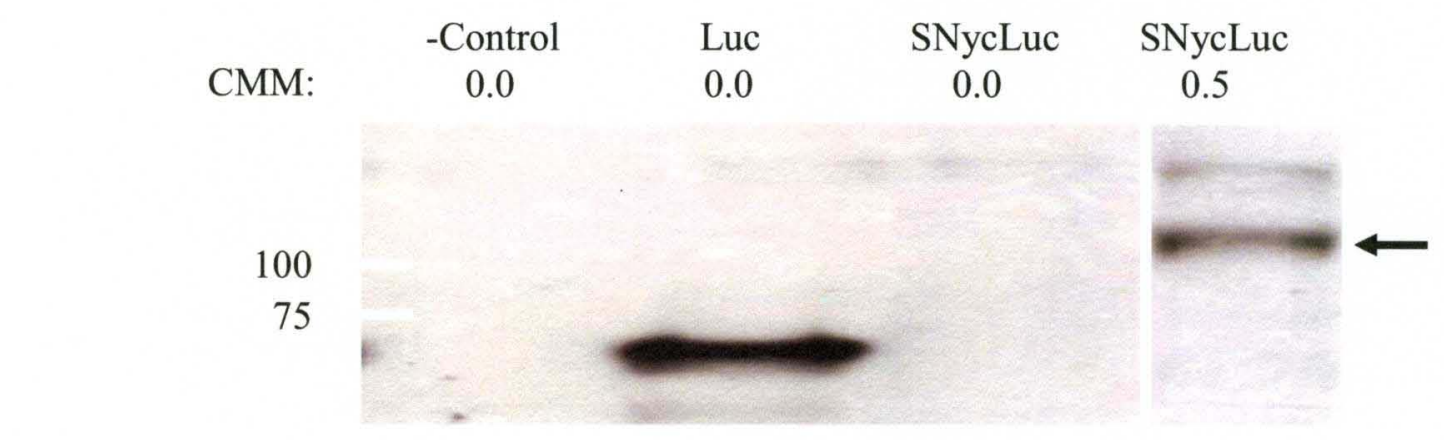

C.

SNycLuc

B.

Lanes

Time (mins)

\begin{tabular}{|c|c|c|c|c|c|c|c|c|c|c|c|}
\hline \multicolumn{4}{|c|}{ Control } & \multicolumn{4}{|c|}{ Prot K $(0.1 \mu \mathrm{g})$} & \multicolumn{4}{|c|}{$\begin{array}{l}\text { Prot K }(0.1 \mu \mathrm{g}) \\
\text { Chaps }(0.5 \%)\end{array}$} \\
\hline 1 & 2 & 3 & 4 & 5 & 6 & 7 & 8 & 9 & 10 & 11 & 12 \\
\hline 5 & 10 & 15 & 20 & 5 & 10 & 15 & 20 & 5 & 10 & 15 & 20 \\
\hline
\end{tabular}

SLucNyc:

SNycLuc: 
Figure 20. N-terminus of nyctalopin is in the lumen of ER. A. LucNyc with the arrows indicating the two thrombin (Thr) cleavage sites. Thrombin cleavage will generate two protein fragments; a $72.8 \mathrm{kDa}$ and a $34.4 \mathrm{kDa}$. The $72.8 \mathrm{kDa}$ will be labeled with biotinylated lysine and detected on western blot. B. Western blot of in vitro translated product before and after thrombin digestion. Disruption of microsomal membranes with chaps allows cleavage of the fusion protein. The band at $72.8 \mathrm{kDa}$ demonstrates that the $\mathrm{N}$-terminus of nyctalopin is protected from thrombin digestion and is therefore in the lumen of the ER. 
A.
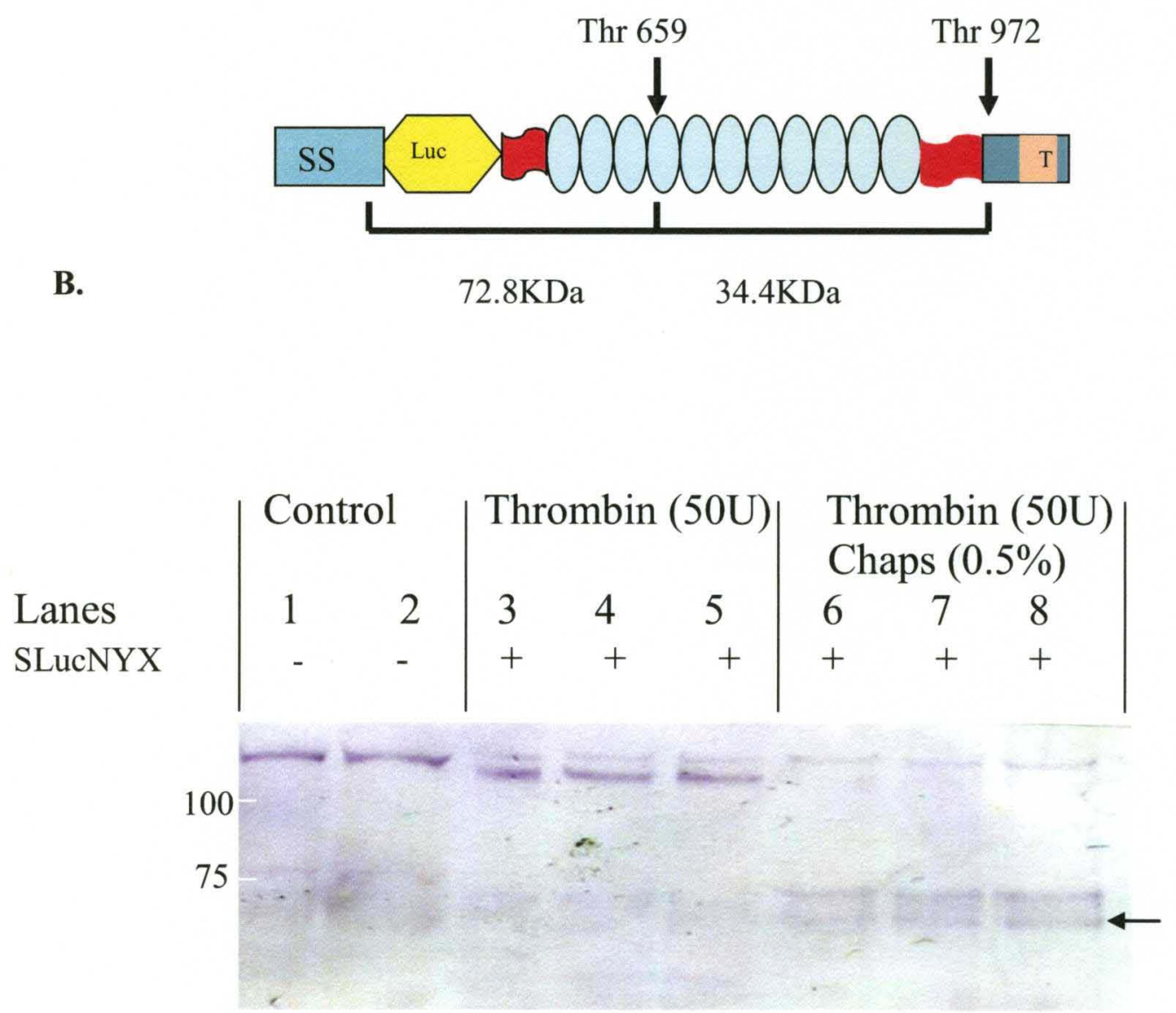
digestion. Both SLucNyc and SNycLuc were tested in this assay. Figure 19C shows that there is no digestion of SLucNyc whereas SNycLuc was digested by proteinase K. This protection is lost when Chaps is added to disrupt the membranes. These results support the conclusion that the $\mathrm{N}$-terminus of nyctalopin is protected and therefore is localized to the lumen of the microsomes and C-terminus of nyctalopin is in the cytoplasm.

The experiment using proteinase $\mathrm{K}$ shows that the $\mathrm{N}$-terminus is protected from digestion. However, after sufficient time proteinase $\mathrm{K}$ can digest all proteins even in the absence of Chaps. To independently confirm that nyctalopin is oriented with the Nterminus in the lumen of the ER and the C-terminus in the cytoplasm. I used a second protease (thrombin) to examine membrane orientation of nyctalopin after in vitro translation. There are two thrombin cleavage sites in SLucNyc, one at position 659 and the other at position 972 (Figure 20A). The yeast two hybrid and proteinase K protection data above suggest that this region of the protein should be in the lumen of the microsomes and therefore protected from thrombin cleavage. Figure 17B shows that in the presence of intact microsomes, SLucNyc is totally protected from thrombin digestion. When the microsomes are disrupted by adding chaps, the protection is lost as can be seen by the generation of the $72.8 \mathrm{kDa}$ cleavage product (Figure 20B). Again these data indicate that nyctalopin is oriented with the LRR domain in the extracellular space.

\section{DISCUSSION}

Data from the topology experiments in yeast and in vitro translation argue against either the absence of a transmembrane domain or of two transmembrane domains for nyctalopin. Alternatively if nyctalopin was GPI anchored then the Cub-LexAVP16 transcription factor should be cleaved from Nyc-Cub fusion protein during the processing 
of GPI anchoring. In both yeast and mammalian cells, GPI anchoring is a post translational process which occurs in the ER (McConville et al., 1993; Ferguson et al., 1999; Ikezawa et al., 2002). It involves the cleavage of the GPI anchor signal sequence and replacing it with a lipid moiety (Caras et al., 1989, Conzelmann et al., 1992). Therefore, if nyctalopin was GPI anchored and the Cub-LexAVP16 transcription was cleaved and released into the cytoplasm, it would have diffused into the nucleus and activated transcription of reporter genes. If the Cub-LexAVP16 transcription factor is degraded, then the topology experiments using Nyc-Cub and Fur-NubI should be negative, which did not occur.

On the other hand if nyctalopin is a secreted protein, the result for Cub-Nyc and Nyc-Cub with Fur4-NubI should both be negative. This is because secreted proteins are secreted into the lumen of the ER which does not contain ubiquitin proteases. Also since the C-terminus and N-terminus of Fur4 are both localized in the cytoplasm, the Cub and NubI cannot come together to form the split ubiquitin molecules. The formation of split ubiquitin molecules is required for the cleavage of the transcription factor. The result for a secreted protein would have been consistently negative.

Human nyctalopin is GPI anchored (Zeitz et al., 2003; Oconnor et al., 2005) while murine nyctalopin is transmembrane bound. Based on the location of the transmembrane domain in murine nyctalopin only four amino acids are in the cytoplasm, suggesting that there might not be functional difference between human and murine nyctalopin. What could be the function of nyctalopin in the signaling cascade from the Grm6 receptor to the Trpml channel? 
In the dark, photoreceptors release glutamate tonically into the synaptic cleft. The glutamate binds to the metabotropic glutamate (Grm6) receptor on depolarizing bipolar cells (Nomura et al., 1994; Vardi et al., 2000) or the AMPA/kainate receptors on hyperpolarizing bipolar cells (Brandstatter et al., 1997; Hack et al., 1999; Devries et al., 2000). Glutamate binding to the Grm6 receptor activates a G-protein signal transduction cascade that closes a non-selective cation channel on the depolarizing bipolar cells (Schiells et al., 1990; Nawy et al., 1991; Yamashita et al., 1991). The cation channel is now known to be Trpm1 (Shen et al., 2009; Morgans et al., 2009; Koike et al., 2009). When there is an increase in light intensity, glutamate release from photoreceptors is decreased. This leads to reduced Grm6 receptor activity, inactivation of the G-protein cascade and opening of the Trpm1 channel, causing depolarization of the depolarizing bipolar cells. Previous data showed that when nyctalopin is absent in depolarizing bipolar cells, the ERG b-wave is lost (Pardue et al., 1998) and more specifically the Trpm1 channel is absent or not gated (Gregg et al., 2007). Topological analysis of nyctalopin showed that the entire leucine rich repeat is in the extracellular space suggesting that nyctalopin cannot be directly involved in the intracellular trimeric G-protein signaling cascade from the Grm6 receptor to the Trpm1 channel.

The leucine rich repeat domain in nyctalopin has been shown to be involved in protein-protein interaction in other SLRP family members (Brandan et al. 2008; Iozzo 1998; Perrimon and Bernfield 2001; Roughley 2006; Schaeferand Iozzo 2008). Several mutations in the leucine rich domain have been shown to cause CSNB1 in human patients (Pusch et al., 2000; Bech-Hansen et al., 2000; Jacobi et al., 2002; O'Connor et al., 2005). Therefore, it is possible that nyctalopin is interacting with the Trpml channel and 
modulating its function. Decorin, which is a family member of nyctalopin, has been shown to interact with epidermal growth factor (EGF) receptor (Izzo et al., 1998). This interaction causes the dimerization and subsequent internalization of the EGF receptor (Izzo et al., 1998; Zhu et al., 2005). The functional form of many transient receptor potential channels is a homo or hetero tetramer (Jiang et al., 2003; Long et al., 2005; Phelps and Gaudet, 2007). Nyctalopin might be assembling the Trpm1 subunits together to form a functional Trpm1 channel. In the $N y x^{n o b}$ mice, Trpm1 is absent at the dendritic tips of the bipolar cells (Pearing and Gregg, unpublished data). It is also possible that nyctalopin is involved in the trafficking of a functional Trpm1 channel to the membrane in depolarizing bipolar cells. I have shown that nyctalopin directly interacts with Apbal (Bojang and Gregg, unpublished data). Apbal is an adapter protein involved in vesicular trafficking of proteins in neurons. 


\section{CHAPTER III}

\section{INTERACTION PARTNERS OF NYCTALOPIN AND ROLE IN Grm6 SIGNALING CASCADE}

\section{INTRODUCTION}

The protein nyctalopin is expressed in the depolarizing bipolar cells of the retina. It is required for signaling from the metabotropic glutamate receptor-6 (GRM6) to transient receptor channel melastatin 1 (Trpm1) (Shen et al., 2009; Morgan et al., 2009; Koike et al., 2009). The exact function of nyctalopin in depolarizing bipolar cells remains to be determined. Murine nyctalopin is an integral membrane bound protein with a single transmembrane domain (Chapter II). The entire leucine rich repeat domain is oriented into the extracellular space (Zeitz et al., 2003; O'Conner et al 2005). The leucine rich repeat is predicted to be involved in protein-protein interaction but the binding partners are unknown. Patch clamp recordings of depolarizing bipolar cells from $\mathrm{Nyx} \mathrm{x}^{\text {nob }}$ mice have provided some clues. When glutamate is puffed on to depolarizing bipolar cells in control retinas, a robust outward current is generated indicating the closure of the Trpm1 channel (Gregg et al., 2007). However, when glutamate is puffed onto depolarizing bipolar cells from $N y x^{n o b}$ mice the outward current is absent. This shows that the Trpml channel cannot be gated in the absence of nyctalopin. To investigate this further, noise analysis was used to determine the open probability of the Trpm1 channel in the $N y x^{n o b}$ 
depolarizing bipolar cell. These results showed that in the absence of nyctalopin the Trpm1 channel is absent or closed (Gregg et al., 2007).

Mammalian transient receptor potential channels (Trp) are comprised of six families and they are divided into two groups. The grouping is based on sequence homology and topological differences (see review by Venkatachalam and Montell, 2007). The major difference is the presence of a large extracellular loop between the first and second transmembrane domains in members of group 2, which is absent in members of group 1. In mammals, group 1 channels are sub-divided into four subgroups and group 2 into two subgroups. The founding member of the Trp channels was identified in Drosophila melanogaster and was called the transient receptor potential channel (Trp) (Montell et al., 1989). The closest mammalian relatives are the canonical/classical Trp or TrpC channels. The names of the other subgroups (subgroup1: TrpV (villanoid), TrpM (melastatin), TrpA (Anktm), and subgroup 2: TrpML (mucolipin), TrpP (polycystin)) are based on the name of the founding member of each subgroup.

Each Trp channel has six transmembrane domains; predicted pore helices located between the fifth and sixth transmembrane domains and have both $\mathrm{N}$ - and $\mathrm{C}$-termini in the cytoplasm. In addition, most Trp channels have a highly conserved 23-25 amino acid stretch called the Trp domain located in the cytoplasm close to the sixth transmembrane domain.

Trp channels are modulated by a diverse array of stimuli ranging from $\mathrm{pH}$, temperature, osmotic pressure and chemicals (Nilius et al., 2005). How these signals are integrated to gate the Trp channels is not fully understood. 
Voltage gated channels are modulated by voltage and share a similar gating mechanism. An important clue to the gating mechanism of these channels came from their protein sequence. These transmembrane domains are positively charged and the location of the positive charges on the S4 helix generates movement of the helix in and out of the membrane when the membrane potential of the cell changes. This movement leads to the opening and closing of these channels (Yellen et al., 1998; Tombola et al., 2006). However, there is no common gating mechanism for Trp channels (Valente et al., 2008; Benjamin et al., 2008). Trp channels have varying degrees of cation permeability. The functional form of Trp channels is a tetramer, which can be either hetero- or homotetramers (Jiang et al., 2003; Long et al., 2005; Phelps and Gaudet, 2007). Given the number of different channel subtypes, not to mention splice variants, this leads to a large number of possible configurations. Also complicating the analysis of Trp channel gating is the lack of highly specific agonists and antagonists.

The non-selective cation channel critical for function in depolarizing bipolar cells, is now identified as the Trpm1 (Shen et al., 2009; Morgans et al., 2009; Koike et al., 2009). Trpm1 was first discovered as a tumor suppressor gene because of its down regulation in metastatic melanoma cells (Duncan et al., 1998). It is the founding member of the melastatin family of Trp channels. The gene is comprised of 27 exons with at least five splice variants in human (Oancea et al., 2009). How the Trpm1 channel is gated in depolarizing bipolar cells is controversial. The Trp channel antagonists, ruthenium red and capsazepine, have been shown to inhibit the Grm6-coupled cation current in depolarizing bipolar cells (Shen et al., 2009). The depolarizing bipolar cell cation current is also sensitive to both capsaicin and anandamide. Capsaicin was thought to be specific 
to Trpvl but examination of $\operatorname{Trpv} 1^{-/-}$mice showed capsaicin mediated responses are still present (Shen et al., 2009). Further, the ERG from these mice is normal, suggesting that the capsaicin response is mediated by another channel now thought to be $\operatorname{Trpm} 1$. Morgans et al. 2009 showed that in the absence of Trpml, they could still record a capsaicin response in some depolarizing bipolar cells. They also showed that pressure application of the Grm6 antagonist, cyclpropyl-4-phosphonophenylglycine (CPPG), (to simulate a flash of light) after bath application of L-AP4 (to simulate darkness) triggered a small transient current in some Trpm $1^{-1-}$ cone depolarizing bipolar cells. In Trpm $1^{-1-}$ mice, the sustained cation current in depolarizing bipolar cells is absent as is the light response (Morgans et al., 2009; Koike et al., 2009). Further, the ERG b-wave is absent in $\mathrm{Trpm}^{-/-}$mice (Shen et al., 2009; Morgans et al., 2009; Koike et al., 2009). These data suggest that the Grm6 mediated cation current consists primarily of a sustained current mediated by Trpm1 and a transient current mediated by an unknown channel. To determine the mechanism by which Grm6 activation gates the Trpm1 channel, Koike and colleagues co-expressed Grm6, Trpm1 and GaO in a heterogeneous system and found that $\mathrm{G} \alpha \mathrm{O}$ is required for the Trpml gating (Koike et al., 2009). They found that if the denatured form of $\mathrm{G} \alpha \mathrm{O}$ was used; the Grm6 dependent regulation of Trpm1 channel was abolished. These data indicate that Trpml gating is mediated by $\mathrm{G} \alpha \mathrm{O}$. However, studies by other groups show that Grm6 regulation of Trpm1 is mediated through the G $\beta \gamma$ subunits (Nawy, 2010; Xu and Vardi, 2010). So it is still unclear exactly how the Trpm1 channel is gated.

Heterogeneous expression studies show that many Trp channels including Trpm1 are constitutively active in vitro (Xu et al., 2001; Oancea et al., 2009; Koike et al., 2009; 
Morgans et al., 2009). Despite the fact that channel activity can be recorded at the plasma membrane, the majority of heterogeneously and endogeneously expressed Trp channels are localized intracellularly (Turner et al., 2003; Bezzerides et al., 2004; Krapivinsky et al., 2006; Dong et al., 2008; Oancea et al., 2009; de Groot., 2009). The dynamic rate of insertion or retrieval of $\operatorname{Trp}$ channel to and from the membrane in response to stimuli embodies the strategic regulation of the Trp channel gating mechanisms (Buckley et al., 2000; Bobanovic., et al. 2002; Montell, 2004).

Trafficking of Trp channels from intracellular organelles is well documented (Buckley et al., 2000; Bobanovic., et al. 2002; Montell, 2004, see reviews by Cayouette and Bouley, 2007; Dong and $\mathrm{Xu}, 2010$ ). In heterogeneous expression systems, the amount of Trpv1/2 in the plasma membrane of CHO and MIN6 cells rapidly increases after presentation of insulin-like growth factor (Kanzaki et al., 1999; Van Buren et al., 2005; Chu et al., 2005). Trpc5 insertion into the plasma membrane requires activation of a receptor tyrosine kinase pathway (Bezzerides et al., 2004). RhoA, a GTP-binding protein, regulates the trafficking of the Trpcl-IP3R complex to the plasma membrane in endothelial cells (Mehta et al., 2003; Ridley, 2001; Jaffe and Hall, 2005). The Drosophila TrpL channel contributes to long term adaptation by shuttling from the rhabdomere membrane to the cell bodies. This is reversed in low luminance conditions (Bähner et al., 2002). In Caenorhabditis elegans, Trpc3 is localized to intracellular vesicles of spermatids and translocates to the plasma membrane during sperm activation $(\mathrm{Xu}$ et al., 2003). In neurons, snare complexes involved in vesicular fusion to the membrane (Jahn et al., 2006) have been shown to interact with Trpc1, Trpc3, Trpv1 and Trpm7 (Itagaki et al., 2004; More Johnssonla-Palao et al., 2004; Krapivinsky et al., 2006). Small G 
proteins that are involved in various steps of vesicular trafficking including vesicle loading, budding, targeting, docking, fusion and motility are involved in the trafficking of Trp channels to the membrane. For example, Rabll is involved in the trafficking of Trpv5 and Trpv6 to the plasma membrane (van de Graaf et al., 2006). The group 1 metabotropic glutamate receptors agonist, (S)-3-5-dihydroxyphenylglycine (DHPG), increases the cytoplasmic levels of $\operatorname{Trpc} 4$ and $\operatorname{Trpc} 5$ and decreases the level of these proteins in the membranes of hippocampal neurons (Wang et al., 2007). This translocation is abolished when hippocampal neurons are deprived of phospholipase $\mathrm{C} \beta 1$ (PLC- $\beta 1$ ) (Chuang et al., 2001; Wang et al., 2007). These data indicate a role of PLC- $\beta 1$ in Trpc4 and Trpc5 translocation to the plasma membrane.

The Trpml channel is localized to both the soma and the dendrites of depolarizing bipolar cells (Morgans et al., 2009, Koike et al., 2009). Heterogeneous expression in HEK293 cells also shows that most of Trpml channels are intracellular (Xu et al., 2001; Oancea et al. 2009). In melanoma cells, Trpml is localized to intracellular vesicular structures (Oancea et al., 2009) and, as a consequence these cells express a very small current. These data raise the possibility that the main function of Trpm1 might be intracellular in melanoma cells. In addition, a splice variant of Trpm1 (Trpm1-s), which lacks all the transmembrane domains has been shown to directly interact with Trpml and suppress its transport to the plasma membrane (Xu et al., 2001). However the intracellular location of Trpml in depolarizing bipolar cells does not correlate with its function as the non-selective plasma membrane bound cation channel required for normal vision. In this context, Trpm1 trafficking to the dendritic tips of depolarizing bipolar cell, might be regulated by a complex of proteins that include nyctalopin. 
In this chapter I investigate the hypothesis that nyctalopin interacts with Trpm1. I show, using the membrane split ubiquitin yeast two hybrid (MYTH) system and in vitro translation combined with immunoprecipitation that they do in fact interact. To determine Trpm1-nyctalopin interacting domains, I fragmented Trpm1 into 3 pieces. MYTH analysis indicates that nyctalopin interacts with both the first and the third extracellular loops. Localization studies of heterogeneously expressed Trpml in yeast show that Trpm1 is not localized to the plasma membrane even in the presence of nyctalopin. Together these data suggest that nyctalopin alone cannot traffic the Trpml channel to the membrane in yeast. I also have identified Apbal as a nyctalopin binding partner. Apbal is an adapter protein involved in vesicular trafficking in neurons. Apbal interacts with Kinesin 17, which is part of a molecular motor. Together these data suggest that Trpm1 trafficking in depolarizing bipolar cells involve a complex of proteins that include nyctalopin, Apba1 and member of the kinesin family of proteins.

\section{METHODS}

\section{Membrane Yeast Two-Hybrid (MYTH) Analyses}

The membrane split ubiquitin yeast two hybrid (MYTH) method was used to determine if nyctalopin interacts with the Trpm1 channel. The MYTH system (described in detail in chapter 2) allows the expression of full length proteins and their insertion into the membrane. This allows protein-protein interactions at the membrane to be examined. A nyctalopin cDNA, without the nucleotide encoding signal sequence (23-476aa), was inserted into the bait vector pCCW-SUC (Dualsystems, Grabenstrasse, Switzerland). Introduction of the plasmid into the yeast strain NMY32 results in the expression of 
nyctalopin fused to the carboxy domain of ubiquitin (Cub) and a LexA-VP16 transcription factor (Nyc-Cub). To analyze interactions with Trpm1, a full length Trpm1 cDNA was cloned into the pDLS-Nx prey vector to create NubG-Trpml. The bait (NycCub) and prey (Trpm1-NubG) were co-transfected into the yeast strain NMY32 using the lithium chloride method (Clontech, Mountain View, CA). The transformed yeast were plated onto SD-LW plates and incubated at $30^{\circ} \mathrm{C}$ for three days. Three independent clones were picked from each plate and spotted onto a fresh SD-LW and SD-LWHA plates. Growth on SD-LWHA plates and activation of the $\beta$-galactosidase gene was dependent on the interaction of nyctalopin and Trpm1 fusion proteins. The expression of $\beta$-galactosidase was assessed by colony lift assay. In the presence of $\beta$-galactosidase, Xgal is converted to galactose and 5-bromo-4-chloro-3-hydroxyindole. The bromo-4chloro-3-hydroxyindole is then oxidized to 5,5'-dibromo-4,4'-dichloro-indigo, an insoluble blue.

\section{Cobalt uptake assay for yeast cultures}

A cobalt assay was used to measure the gating properties of the Trpm1 channel in yeast (Myers et al., 2007). A single BY4741 yeast colony expressing either Trpv1, Kir2.1, Trpm1 or Trpm1 plus nyctalopin was inoculated into synthetic liquid dropout medium (i.e. $\mathrm{SD} /$-Leu or $\mathrm{SD} / \mathrm{Leu} / \mathrm{Trp}$ ) and grown overnight to saturation. The optical density at $600 \mathrm{~nm}\left(\mathrm{OD}_{600}\right)$ was measured and each culture was diluted to $0.1 \mathrm{OD}_{600}$ and grown in $50 \mathrm{ml}$ of synthetic liquid dropout medium to mid-log $\left(\mathrm{OD}_{600}=0.5-0.7\right)$. The cells were pelleted by centrifugation at $700 \mathrm{~g}$ for 5 minutes and washed once in $25 \mathrm{ml}$ of cobalt uptake assay buffer $\left(58 \mathrm{mM} \mathrm{NaCl}, 5 \mathrm{mM} \mathrm{KCl}, 2 \mathrm{mM} \mathrm{MgCl}_{2}-6 \mathrm{H}_{2} \mathrm{O}, 0.75 \mathrm{mM} \mathrm{CaCl}_{2}, 12 \mathrm{mM}\right.$

glucose, $137 \mathrm{mM}$ Sucrose and $10 \mathrm{mM}$ HEPES, $\mathrm{pH}$ 7.4). The cells were re-suspended in 
$10 \mathrm{ml}$ of cobalt uptake buffer and $500 \mu \mathrm{l}$ was aliquoted into each well of a 96 well plate. The cells were pelleted at $14000 \mathrm{~g}$ on a bench top centrifuge for 30 seconds, the supernatant was removed and the pellet was re-suspended in $500 \mu l$ of cobalt uptake buffer plus $5 \mathrm{mM} \mathrm{CoCl}$. Pharmacological agents were also added at this time. The samples were incubated at $30^{\circ} \mathrm{C}$ for 5 minutes to stimulate uptake, then placed on ice for 1-2 minutes. Samples were washed twice with $500 \mu 1$ of cobalt uptake assay buffer. Each sample was re-suspended in $500 \mu 1$ of $1 \%$ ammonium sulfide dissolved in cobalt uptake buffer and incubated at room temperature for 3 minutes. The cells were pelleted and the wash step was repeated using $100 \mu l$ cobalt uptake buffer. If cobalt is present in the yeast cells cobalt sulfide (CoS) forms, which turns yeast cells black. The pelleted cells were scanned to estimate the amount of cobalt sulfide $(\mathrm{CoS})$ formed.

\section{Interaction of nyctalopin and Trpm1 by Co-immunoprecipitation}

The interaction of nyctalopin and Trpm1 was determined by using immunoprecipitation after in vitro translation. An in vitro coupled transcription and translation system supplemented with canine microsomal membrane was used according to the manufacturer's instructions (Promega, Madison, WI, USA). At the end of the 90 minutes

reaction, samples were incubated on ice. One $\mathrm{ml}$ of immunoprecipitation buffer $(50 \mathrm{mM}$ Tris-horizontal celll ( $\mathrm{pH} 7.5$ ), $150 \mathrm{mM} \mathrm{NaCl}, 0.2 \mathrm{mM} \mathrm{Na}_{3} \mathrm{VO}_{4}, 0.5 \%(\mathrm{w} / \mathrm{v})$ Nonidet P-40, $1 \mathrm{mM}$ phenylmethylsulfonyl fluoride) and protease inhibitor cocktail (Roche, Mannheim, Germany) (one tablet $/ 10 \mathrm{ml}$ ) was added to each sample, mixed and incubated on ice for 30 minutes. $20 \mu 1$ of protein G-agarose (PAGA) (Roche, Mannheim, Germany), prewashed three times in immunoprecipitation buffer, was added to each sample and incubated for 4 hours at $4^{\circ} \mathrm{C}$ with rotation. PAGA was pelleted by centrifugation at 
$14,000 \mathrm{~g}$ for 3 minutes in a bench-top centrifuge. This removes any proteins that had interacted non-specifically with PAGA. The supernatant containing translated labeled nyctalopin (SlucNyc) was mixed with supernatant containing unlabelled Trpm1-loopl (Trpm1-Lpl) or controls. For the reverse co-immunoprecipitation, Trpml-Lp1 was labeled while SlucNyc was not labeled. The combined supernatants were then incubated with $2.5 \mu \mathrm{g}$ of the requisite antibody ( $\alpha \mathrm{HA}$ or $\alpha \mathrm{Luc}$ ) overnight at $4^{\circ} \mathrm{C}$ with rotation. $20 \mu \mathrm{l}$ of PAGA was added to each sample and incubated at $4^{\circ} \mathrm{C}$ for an additional 60 minutes. The protein antibody conjugate bound to PAGA were recovered by centrifugation at 14 , $000 \mathrm{~g}$ for 3 minutes, washed in $1 \mathrm{ml}$ of Buffer A (phosphate-buffered saline, $0.2 \%(\mathrm{w} / \mathrm{v})$ Triton $\mathrm{X}-100$ and $350 \mathrm{mM} \mathrm{NaCl}$ ). The pellets were re-suspended in $1 \mathrm{ml}$ of Buffer $\mathrm{B}$ (phosphate-buffered saline, $0.2 \%(\mathrm{w} / \mathrm{v})$ Triton $\mathrm{X}-100)$, centrifuged, and re-suspended in SDS sample buffer. The samples were resolved by electrophoresis on $4-12 \%(\mathrm{w} / \mathrm{v})$ polyacrylamide gels. Proteins were transferred to Hybond ${ }^{\mathrm{Tm}}$-LFP Transfer Membrane (GE Healthcare, UK) and bands visualized by reacting with streptavidin conjugated alkaline phosphatase (Strep-AP), which binds to the biotin labeled proteins.

\section{Channel expression and localization in yeast cells}

A single BY4741 yeast colony expressing either Kir2.1- EYFP or Trpm1-EYFP under

the glyceraldehyde-3-phosphate dehydrogenase (GDP) promoter was inoculated in synthetic dropout medium (i.e. $\mathrm{SD} /-\mathrm{Leu}$ ) and grown to an $\mathrm{OD}_{600}$ of 1.0. Cells were then diluted tenfold $\left(\mathrm{OD}_{600}=0.1\right)$ and grown to mid-log $\left(\mathrm{OD}_{600}=0.5-0.7\right)$ before fixing them in $100 \mathrm{mM}$ phosphate buffer ( $\mathrm{pH} 6.5$ ) containing $3.7 \%$ formaldehyde at room temperature for 2 hours. The cells were washed twice in distilled $\mathrm{H}_{2} \mathrm{O}$ and re-suspended in $1 \mathrm{ml}$ of 
$\mathrm{H}_{2} \mathrm{O}$. Three microliters of the re-suspended cells were added to a microscope slide and covered with a cover slip. The fluorescence of the yeast cells was viewed using an Axiovert 25 CFL microscope equipped with $\mathrm{HBO} 50$ illuminator and fluorescence reflector mount. Images were acquired and analyzed using the Axiovision Release 4.3 software.

\section{cDNA library construction}

First strand cDNA was synthesized using the Plugoligo-3M primer (5'-GCAGTGG TATCAACGCAGAGTGGCCATTACGGCCGGGGG-3') and PolyAPrimer (5'-CGAA TTCTCGAGAGGCCGAGGCGGCCGACATGTTTTTTTTTTTTTTT-3') primers that I designed in accordance with the manufacturer's protocol (Dualsystem, Grabenstrasse, Switzerland). The Plugoligo-3M and PolyAprimer primers each have a unique Sfil site 5'-GGCCATTACGGCC-3' and 5'-GGCCGAGGCGGCC-3' respectively, which allowed cloning into the SfiI site of the pDLS-Nx prey vector (Dualsystem, Grabenstrasse, switzerland). First strand cDNA was synthesized using a Moloney Leukemia Virus reverse transcriptase (MMLV-RT) in the presence of the PolyAprimer. When MMLV-RT reaches the 5'-terminus of the RNA template, the enzyme terminal transferase activity adds a few deoxycytidines to the 3' end of the cDNA. The Plugoligo3M, which has oligo G sequence at the 3 ' end hybridizes with the deoxycytidine stretch. MMLV-RT then switches template and synthesize the second strand of cDNA (Figure 21A). To amplify the double stranded cDNA, I used long distance PCR (LDPCR) with the 5'-Primer (5'- CGCTGGATCCAAGCAGTGGTATCAACGCAGAGTGGCCATT ACGGC-3') and 3'-Primer (5'-TATCGATAAGCTTGATATCGAATTCTCGAGAGG CCGAGGCGGCCG-3'). To find out the optimal cycle number several samples were 
amplified with varying cycle numbers $(18,19,20)$ (Figure 21B). An optimal PCR cycle number was 18 because of the distinct bands and the even size distribution of the PCR

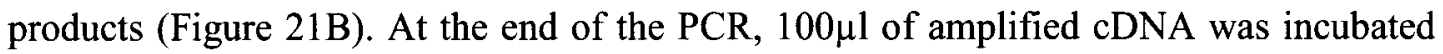
with $1 \mu \mathrm{l}$ of proteinase $\mathrm{K}(20 \mu \mathrm{g} / \mu \mathrm{l})$ at $45^{\circ} \mathrm{C}$ for 20 minutes. The cDNA library was phenol/chloroform/isoamyl alcohol $(25: 24: 1)$ extracted and precipitated with $3 \mathrm{M}$ sodium acetate $(10 \mu \mathrm{l}), 20 \mu \mathrm{g} / \mu \mathrm{l}$ of glycogen $(1.3 \mu \mathrm{l})$ and $260 \mu \mathrm{l}$ of room temperature $95 \%$ ethanol. The cDNA was washed once with $80 \%$ ethanol then dried at room temperature for 10 minutes. The pellet was resuspended in $79 \mu \mathrm{l}$ of nuclease free $\mathrm{H}_{2} \mathrm{O}$. The amplified cDNA was then digested with SfiI $(10 \mu \mathrm{l})$ overnight. CHROMA SPIN ${ }^{\mathrm{TM}}-400$ (Clontech, Mountain View, CA) columns were used to remove small cDNA fragments. Afer size fractionation, the cDNA library was precipitated and resuspended in $7 \mu 1$ of deionized $\mathrm{H}_{2} \mathrm{O}$. To make the library, varying amounts cDNA library $(0.5 \mu \mathrm{l}, 1.0 \mu \mathrm{l}, 1.5 \mu \mathrm{l})$ were ligated into 500ng of SfiI linearized pDLS-Nx vector overnight at $16^{\circ} \mathrm{C}$ using T4 DNA ligase (New England Biolabs, Ipswich, MA) in a final volume of $20 \mu 1$. A test transformation using $1 \mu 1$ of the ligation mix was transformed into chemically competent Top10 E. coli cells. pUC19 plasmid $(10 \mathrm{pg} / \mu \mathrm{l})$ was used as transformation control. To asses of the complexity of the cDNA library, number of independent transformants, size of the insert, and the number of clones in the library was calculated. Figure 22 shows an outline of this process. The final library was generated by transforming the remaining ligation mix into $E$. coli Top10 cells and plated onto 200 Ampicillin containing LB plates $(100 \mu \mathrm{g} / \mu \mathrm{l})$. After overnight incubation, LB-medium $(5 \mathrm{ml})$ containing Ampicillin $(100 \mu \mathrm{g} / \mu \mathrm{l})$ was added to each plate, bacteria were resuspended and samples for all 200 plates pooled. Plasmids were isolated from the bacterial suspension using the Cesium 
chloride method. To identify interacting proteins the purified plasmids were transformed into NMY32 strain already transfected with Nyc-Cub. Transformed yeast were plated onto twenty $\mathrm{SD} /$-Leu/Trp/His (triple dropout) plates and twenty $\mathrm{SD} /$-Leu/Trp/His/Trp (quardruple dropout) plates and incubated at $30^{\circ} \mathrm{C}$ for three days. Clones were picked and prey and bait plasmids were isolated from yeast using the SV Mini prep (Promega, Madison, WI) with the addition of acid washed glass beads to disrupt the yeast cells. The purified plasmid DNA was re-transformed into bacteria Top10 E. coli cells and plated onto Ampicillin containing plates to isolate the prey plasmids. The plasmids were purified from bacterial colonies as described above, the size of the insert determined using restriction digestion and agarose gel electrophoresis and the insert sequenced. Both BLAT (UCSC genome browser) and Blast (NCBI) software programs were used to determine the identity of the interacting prey protein. An outline of this process is shown in Figure 23. 
Figure 21. Methodology used to generate retina cDNA for the library. A. First strand synthesis followed by long distance PCR. This process creates single stranded cDNA with Plugoligo-3M primer and PolyAPrimer as 5' and 3' end anchors, respectively. B. Agarose gel of the amplified cDNA. The product from the most representative PCR was used to make the library. Optimal PCR cycle number was 18 because of the even size distribution and the distinct bands. 


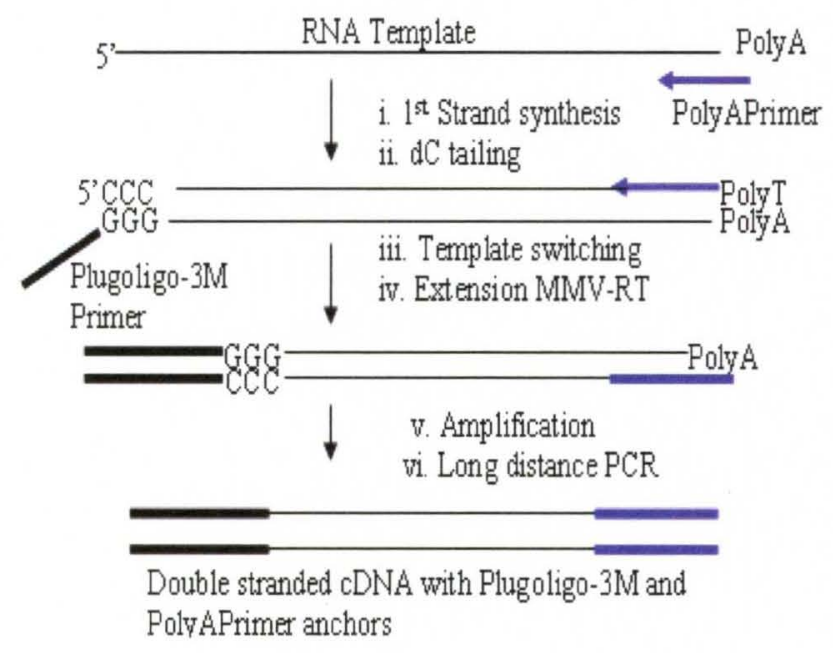

Long Distance PCR

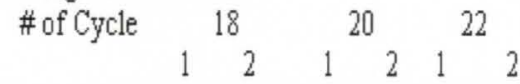

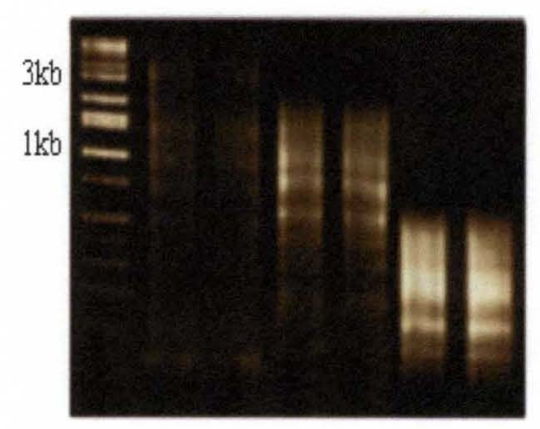


Figure 22. Retinal cDNA library Construction. The cDNA generated by long distance PCR was digested with the restriction enzyme, Sfil. The enzyme is inactivated by proteinase $\mathrm{K}$ and the cDNA purified by phenol/chloroform extraction and ethanol precipitated. To determine the optimal ratio for ligation of the cDNA library and linearized $\mathrm{pDLS}-\mathrm{Nx}$ vector, three test ligation reactions were run with varying ratios of cDNA/vector. Ligated plasmids were transformed into Top10 E. coli cells; seven clones were picked from each plate and analyzed after SfiI restriction digestion. The optimal ratio was $0.5 \mu 1$ of $\mathrm{cDNA}$ and $500 \mathrm{ng}$ of linearized $\mathrm{pDLS}-\mathrm{Nx}$ vector. This was chosen because $100 \%$ of the clones tested had inserts. This ratio $(0.5 \mu 1$ library/500ng pDLS-Nx) was used to do ten independent ligation reactions. 

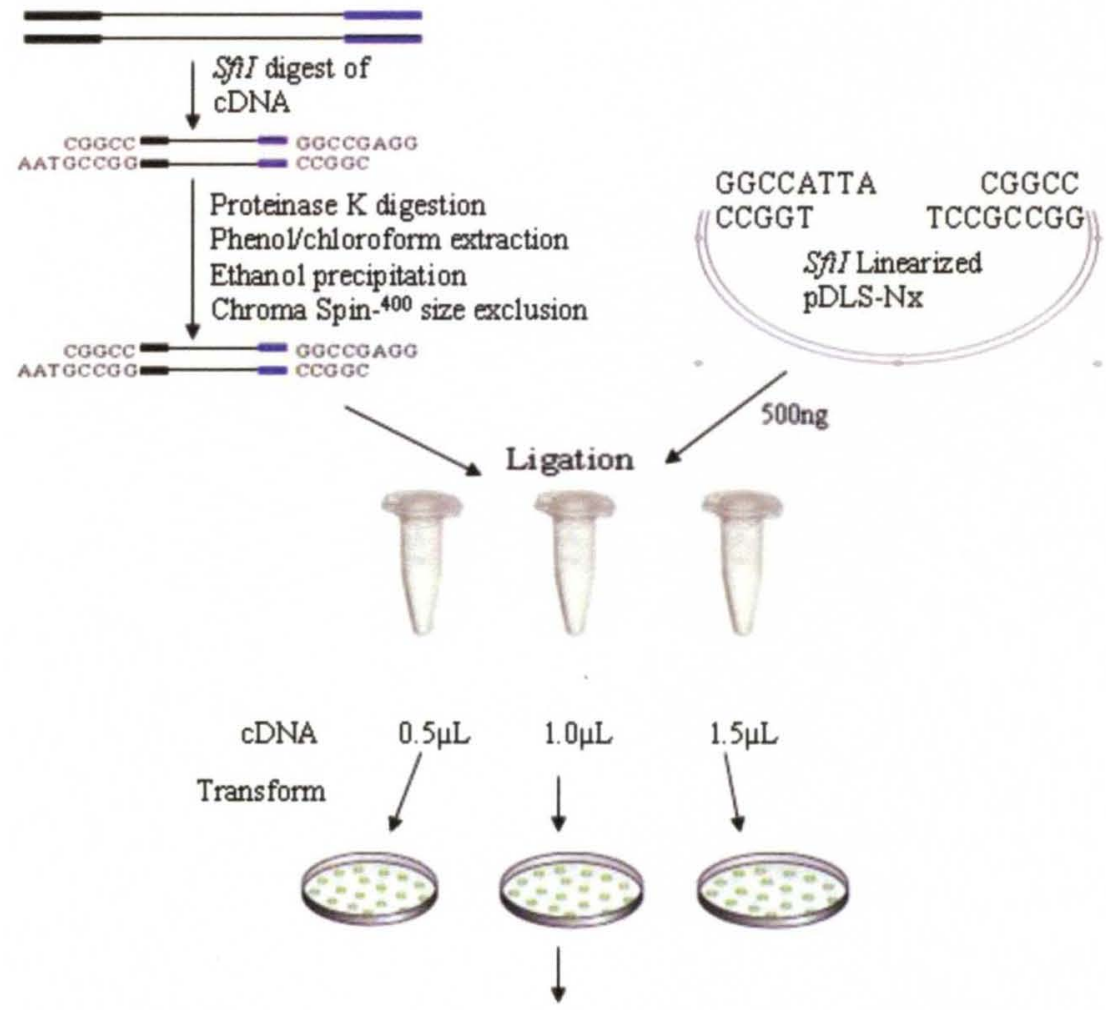

$\underline{0.5 \mu L \text { library }}$

$1.0 \mu \mathrm{L}$ lbrary

$\underline{1.5 \mu L \text { Library }}$

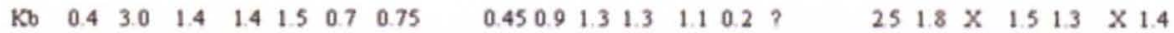

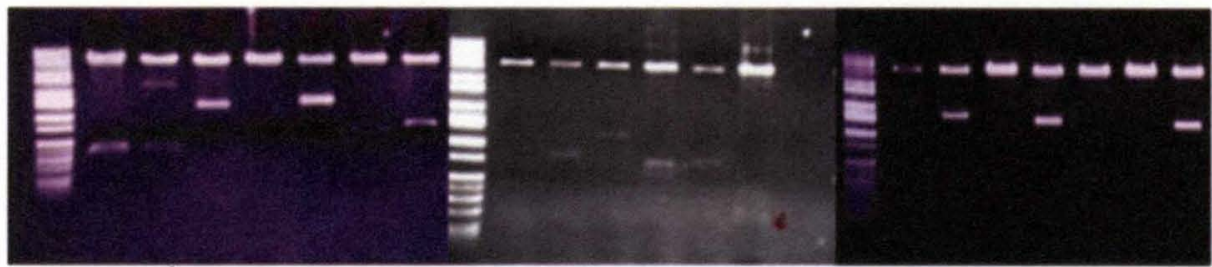

Insert/Nector

$7 / 7$

$6 / 7$

$4 \pi$ 
Figure 23. Membrane split ubiquitin Yeast two hybrid analysis. The ligated library was transformed into Top 10 E. coli cells, plated on 200 plates $(150 \mathrm{~mm}$ X $150 \mathrm{~mm}$ plate) and incubated at $37^{\circ} \mathrm{C}$ overnight. The next morning, all the clones from the 200 plates were pooled together by pouring $5 \mathrm{ml}$ of $\mathrm{LB}$ meduim into each plate and shaking to dislodge and resuspend the clones. The plasmids were isolated from a $250 \mathrm{ml}$ aliquot by the Cesium chloride method. For the yeast two hybrid screen, $7 \mu \mathrm{g}$ of the purified plasmids were transformed into competent yeast NMY32 strain that contained the bait vector (Nyc-Cub). Transformed yeast were plated onto twenty plates of SD/-LWH and twenty plates of SD/-LWHA. These plates were incubated at $30^{\circ} \mathrm{C}$ for three days, then bait and prey plasmids were isolated from each yeast clone, re-transformed into Top $10 \mathrm{E}$. coli cells and plated on ampicillin containing plates to select for the prey plasmid. SfiI restriction digest was performed to determine the insert size and those with inserts were sequenced. 


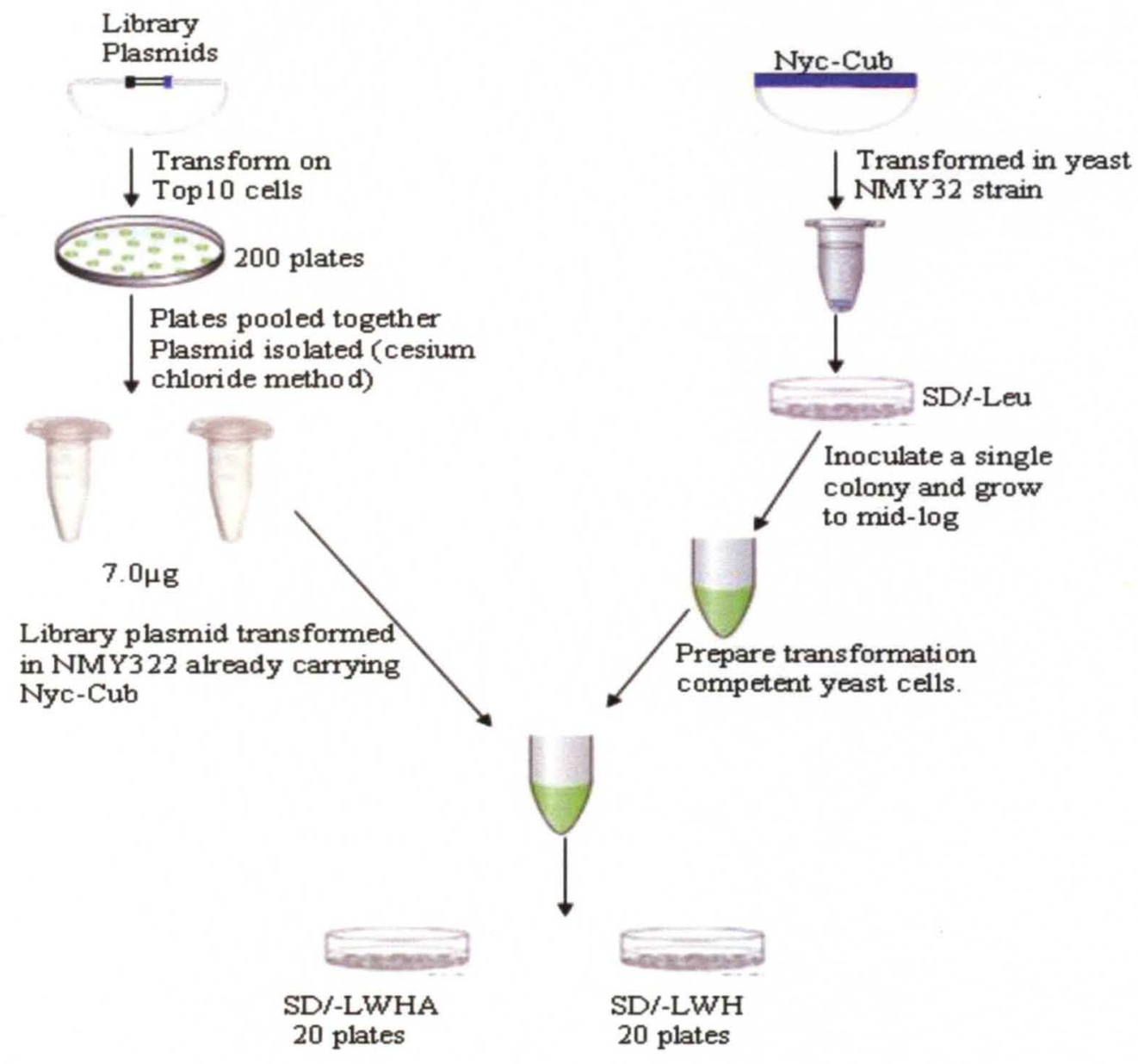

Clone \#:

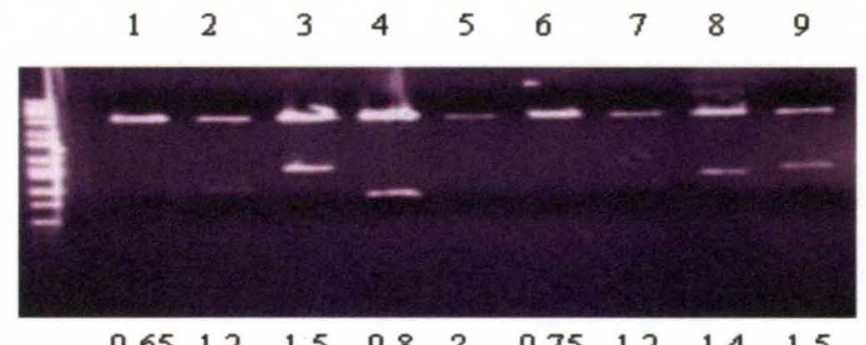

Insert Size:

$\begin{array}{llll}0.65 & 1.2 & 1.5 & 0.8\end{array}$

$\begin{array}{llll}0.75 & 1.2 & 1.4 & 1.5\end{array}$ 


\section{RESULTS}

\section{Nyctalopin interacts with Trpm1}

I have shown that the mRNA level of the Trpml in the $N y x^{n o b}$ mouse retina is decreased at postnatal day 13 (Appendix). Previous data from the Gregg lab has also showed that in the absence of nyctalopin, the Trpm1 channel is either absent or constitutively closed (Gregg et al., 2007). To determine if nyctalopin can directly interact with Trpm1, I used the membrane split ubiqutin yeast two hybrid system. A plasmid encoding nyctalopin (Nyc-Cub) and Trpml (Trpm1-NubG) fusion proteins was transiently transfected into the yeast NMY32 strain and their interaction determined by growth assays and activation of the $\beta$-galactosidase gene, which is indicated by the formation of blue coloration. As a positve control for interaction, Grm6 and $\mathrm{GaO}$ were used. Figure 24, column 1 shows that both plasmids are incorporated into the yeast. The second and third columns show whether there are interaction of the bait and prey proteins. The interaction is indicated by growth on the quadruple dropout plate (SD-LWHA) (column 2) and a positive $\beta$ galactosidase assay (column 3). Grm6 and $\mathrm{G} \alpha \mathrm{O}$ (row 1) show interaction as expected by both assays. Trpm1 and Grm6 (row 2) shows no interaction as there is a lack of growth on quadruple dropout plate (SD-LWHA) and absence of blue coloration in the $\beta$ galactosidase assay in column 2 and 3. However, Trpm1 and nyctalopin (row 3) show interaction since there is both growth and the formation of blue coloration in the $\beta$ galactosidase assay.

\section{Nyctalopin interacts with Loop1 and Loop3 of Trpm1}

To determine what region of Trpm1 is interacting with nycatalopin, I made Trpm1 truncations (Loop1, Loop2, Loop3) and tested their interaction with nyctalopin 
Figure 24. Nyctalopin interacts with Trpm1 in the MYTH system. MYTH analyses of full length nyctalopin (Nyc-Cub) co-transformed with prey vectors expressing Trpm1 (Trpm1-NubG) show that the two proteins interact. There is no interaction of Trpm1 and Grm6. GaO and Grm6 were used as positive controls. 


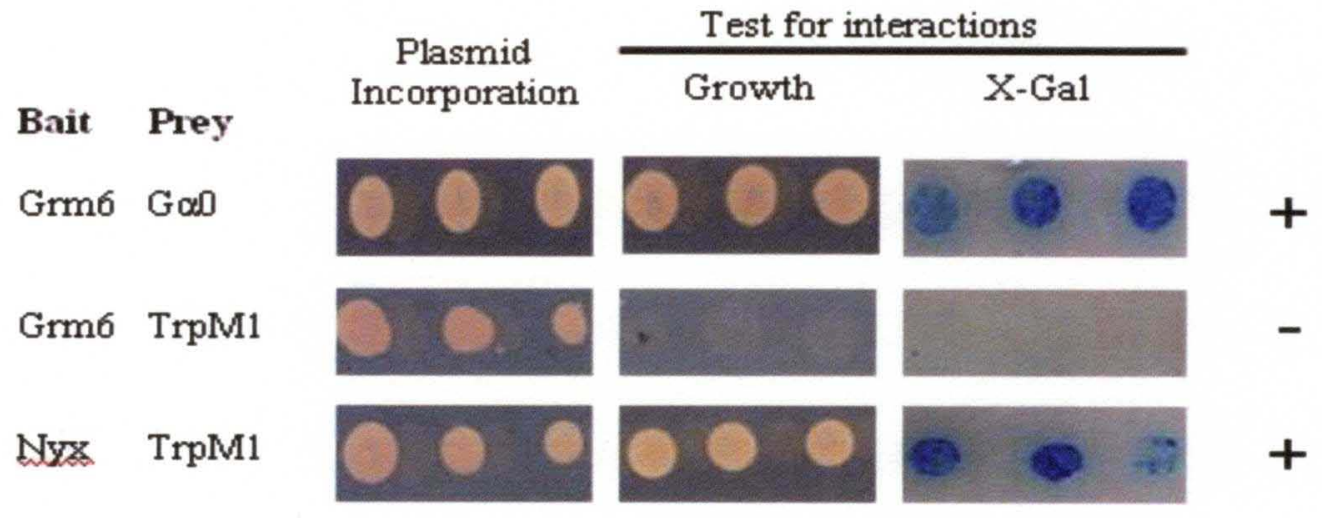


(Figure 25A). Figure 25A shows that both Loop1 and Loop3 interacted with nyctalopin as indicated by growth on SD-LWHA plates and the formation of blue coloration (column 2, row 1 and 3). Growth as well as $\beta$-galactosidase activation is negative when Loop2 is used, indicating that loop2 does not interact with nyctalopin in the MYTH system.

To further substantiate the interaction between nyctalopin and Trpm1, I did coimmunoprecipitation assays using nyctalopin (SlucNyc) and HA-tagged Loop1 of Trpm1 (HA-Trpm1-Lp1). Co-immunoprecipitations were performed from proteins synthesized in cell free in vitro transcription/translation system supplemented with canine microsomal membrane. I labeled nyctalopin (SlucNyc) with biotin using transcend tRNA (Figure 25B) while HA-Trpm1-Lp1 was not labeled. The biotin labeled nyctalopin was incubated with lysate of unlabeled HA-Trpm1-Lp1. As a control unlabeled HA-Trpm1-Lp1 was incubated with lysates of unrelated labeled protein (luciferase) or translated/untranslated empty rabbit reticulocyte lysates. The interacting protein complex was immunoprecipitated with anti-HA antibody (Figure 25B). The first four lanes of Figure 25B show that the translation assay produced the expected product. The last lane of Figure 25B shows that HA antibody to HA-Trpm1-Lp1 can immunoprecipitate the biotin labeled nyctalopin. No band was seen when an un-related protein, luciferase (Luc), and HA-Trpm1-Lpl was immunoprecipitated with the HA antibody. These results indicate that the interaction between nyctalopin and HA-Trpm1-Lp1 is not due to non-specific interactions. Rabbit reticulocyte lysates without addition of plasmids was used as a control to determine if Trpm1-Lp1 is interacting with another protein with the same molecular weight as nyctalopin (SlucNyc). Co-immunoprecipitation of HA-Trpm1-Lp1 
with the HA antibody did not also yeild any non-specific bands corresponding to the size of nyctalopin. To determine if the interaction is caused by conjugation of the HA antibody to the nyctalopin-Trpm1-Lpl complex, co-immunoprecipitation was done in the absence of HA antibody and again no band was seen. From these data, I conclude that Trpm1-Lp1 can interact with nyctalopin.

To determine if the reverse is true, I did co-immunoprecipitation after translating and labeling Trpm1-Lp1 with biotin and SlucNyc was unlabeled. The first four lanes of Figure $25 \mathrm{C}$ indicate the unlabelled nyctalopin-luciferase (SlucNyc) fusion protein is expressed and recognised by the antibody for luciferase. Luciferase expression (Figure $25 \mathrm{C}$, lane 1-2) was used as positive control. Figure 25C indicates that nyctalopin can coimmunoprecipitate Trpm1-Lp1 as indicated by a $114 \mathrm{kDa}$ band. In the absence of TrpmLp1, nyctalopin does not non-specifically interact with another protein from the translation reactions (Figure 25C). Together these data clearly indicate that nyctalopin interacts with Trpm1-Lp1.

\section{Nyctalopin alone cannot regulate the function of the Trpm1 channel}

The Grm6 coupled cation channel in bipolar cells has been shown to be blocked by both ruthenium red and capsazepine (Shen et al., 2009). This channel was also shown to be activated by both capsaicin and anandamide (Shen et al., 2009). Given that Trpm 1 is the Grm6 coupled channel in depolarizing bipolar cells, I examined whether Trpm1 expressed in yeast could be modulated by these same pharmacological agents, and whether co-expression with nyctalopin modulated activity.

A cobalt uptake assay that is based on the formation of cobalt sulfide (CoS), a black precipitate was used (Myers et al., 2007). In aqueous conditions, cobalt chloride 
dissociates into $\mathrm{Co}^{2+}$ and $\mathrm{Cl}^{-}$ions. The $\mathrm{Co}^{2+}$ ions can pass through cobalt permeable channels that are heterogeneously expressed at the plasma membrane of yeast cells (Myers et al., 2007). Sulfide ions, which freely diffuse through the cell walls of yeast cells react with the cobalt ions to form a cobalt sulfide precipitate, which turn yeast cells black. To test the hypothesis that a combination of pharmacological agents and nyctalopin can modulate Trpm1 gating, I transfected yeast BY4741 cells with plasmids encoding Trpv1 (positive control), Kir2.1 (negative control), Trpml and Trpm1 plus nyctalopin. Transfected yeast cells were grown to mid log phase $\left(\mathrm{OD}_{600}=0.5-0.7\right)$ and treated with cobalt choride and ammonium sulfide in the presence or absence of various agonists and antagonists $(10 \mu \mathrm{M})$ (Figure 26A). Figure 26A, column 1, shows the result for Trpv1. No black precipitate (CoS) forms in the absence of pharmacological agents indicating that cabalt ions do not freely permeate the yeast cells and the heterogeneously expressed channels are not constitutively open. Addition of capsaicin and anandamide (column 1, rows 3 and 4 respectively) results in the formation of a black CoS precipitate, which could be blocked by additon of ruthenium red (column 1, row 5 and 6 ). To ensure specificity of the assay and the agonist, a potassium channel Kir2.1, was used as negative control (column 2). All lanes in column 2 show the absence of cobalt uptake, indicating that the results for Trpvl are specific to the presence of the channel at the membrane. There is no cobalt uptake under any condition when Trpm1, in the absence (column 3) or presence of nyctalopin (column 4) is expressed. These data indicate that Trpm1 is either not sensitive to capsaicin and anandamide, contrary to depolarizing bipolar cell patch clamp data (Shen et al., 2009) or Trpml is not localized to the plasma membrane. 
Figure 25. Nyctalopin interacts with Loop 1 and 3. A. Further dissection of Trpm1 indicates that nyctalopin interacts with the extracellular loops between transmembrane domains 1 and 2 and also 5 and 6 but not the 3 and 4 region. B. Co-immunoprecipitation showing that Trpm1-lpl can co-precipitate nyctalopin (last lane). C. Reverse coimmunopreciptation indicating that nyctalopin can co-precipitate Trpml-lp1. Star (*) indicates the protein that is labeled with biotin using transcend tRNA. SlucNyc is nyctalopin with luciferase inserted after its signal sequence. Trpm1-lp1 is first loop of Trpm1. Luc is luciferase, it is used as a control for non-specific interaction in $\mathbf{B}$ and specificity of the luciferase antibody in C. Lysate is unprogrammed rabbit reticulocyte lysate. Plus sign $(+)$ indicates the addition of reagent into the in vitro transcription/translation mix. 
A.

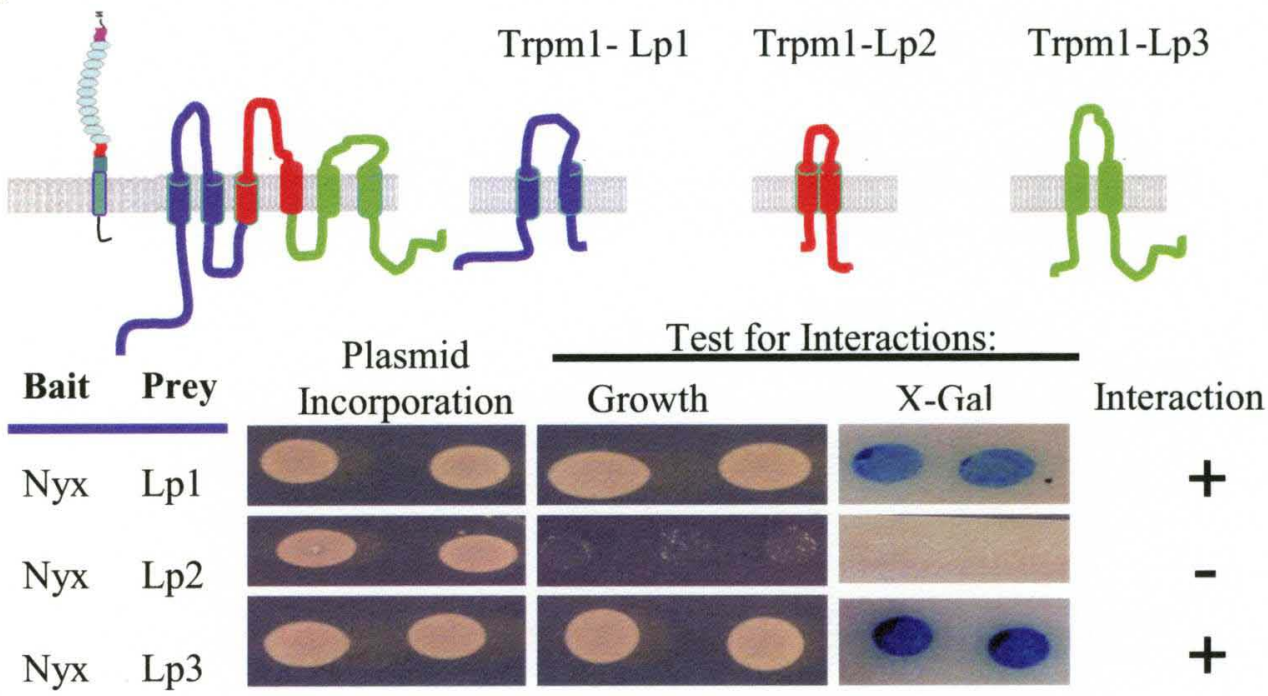

B.

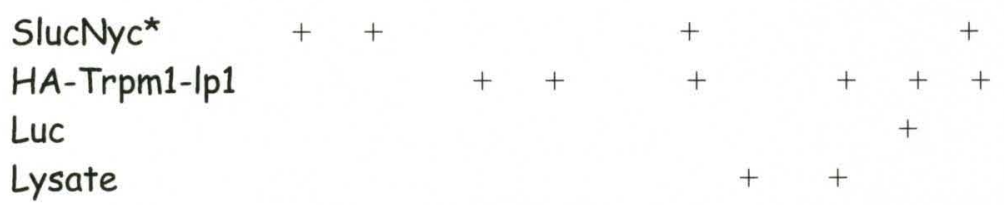

100

75
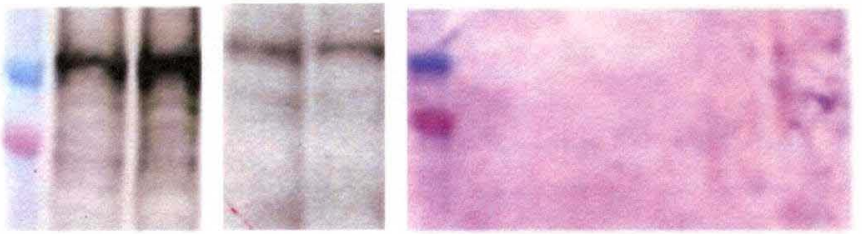

IP: Anti-HA

IB: Strep-AP

C.

HA-Trpm1-Lp1*

SlucNyc

Luc

Lysate
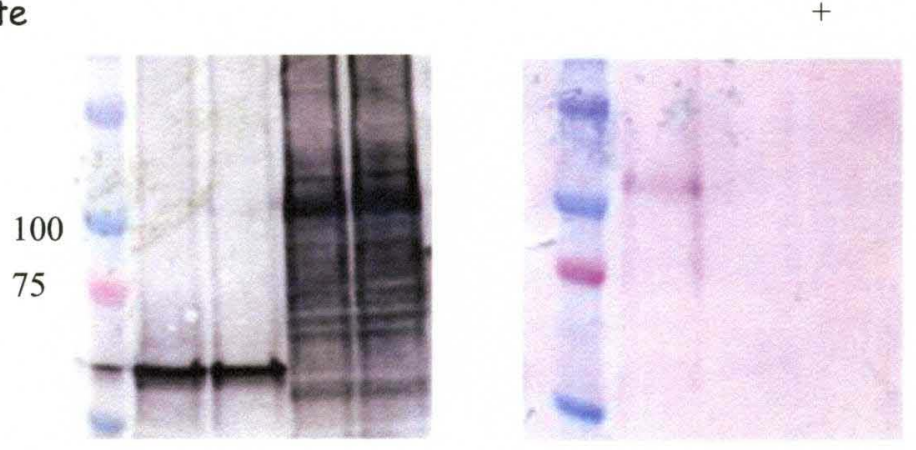

IP: anti-Luc

IB: Strep-AP 
To determine if Trpm1 is localized to the plasma membrane, I tagged Trpm1 with enhanced yellow fluorescence protein (EYFP), and determined its localization by fluorescence microscopy. Figure 26B, compares the localization of the control EYFPKir2.1, and EYFP-Trpm1. The staining for EYFP-Kir2.1 is consistant with expression at the plasma membrane. The staining of EYFP-Trpm1 shows intracellular localization but little evidence for expression at the plasma membrane. This appears to indicate Trpm1 is present at the ER membrane.

To determine if Trpml is present in the yeast ER membrane, I used the membrane split ubiquitin yeast two hybrid system. I co-expressed Trpm1-Cub/CubTrpml with NubI tagged yeast proteins with Alg5-NubI and Fur4-NubI, which are expressed at the ER membrane and plasma membrane, respectively. Co-expression of Alg5-NubI and Trpm1-Cub results in positive growth on SD-LWHA and positive $\beta$ galactosidase assay (Figure 26C-i). However when Trpm1-Cub or Cub-Trpm1 were coexpressed with Fur4-NubI, both growth and $\beta$-galactosidase assays were negative. This indicates that Trpml is localized to the ER membrane.

It was reported that Trpm1 channels are constitutively open when expressed in HEK293 cells (Oancea et al. 2009, Koike et al., 2009). To make sure that Trpm1 channels are not constitutively open to calcium overload and killing the yeast cells, I added capsazepine to the plates. Capsazepine has been shown to block the Trpml channel in depolarizing bipolar cell (Shen et al., 2009). Addition of capsazepine to the plates did not change the result. Both growth and $\beta$-galactosidase assays were negative (Figure 28C-ii). Next, I determined if the truncated forms of Trpm1 can reach the plasma 
Figure 26. Functional analysis of Trpm1 in yeast cells. A. cobalt assay indicating that compared to Trpv1, Trpml gating is not modulated by either capsaicin or anandamide. B. Fluorescence microscopy of yeast cells indicates that compared to the potassium channel (Kir2.1), Trpm1 is not localized to the plasma membrane in yeast. C. Co-localization studies using the MYTH system. i. Trpm1 is present in the ER membrane as indicated by growth and $\beta$-galactosidase assays. ii. Trpm1 is not present in the plasma membrane as indicated by absence of growth and negative X-gal assay. Addition of capsazepine to the plate makes no different indicating the negative growth and X-gal assay is not due to constitutively activite Trpm 1 channel at the membrane. iii. Both truncated forms of Trpm1 (Lp1 and Lp3) are present in the membrane. 
A.

$\begin{array}{ccc}\text { Trpyl Kir2.1 } & \text { Trpml } & \text { Trpml } \\ & & + \\ & & \text { Nyx }\end{array}$

No $\mathrm{CoCl}_{2}$

No Drug

Caps

Ana

Caps + RR

$\mathrm{Ana}+\mathrm{RR}$

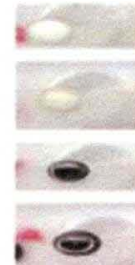

年

C.

i.

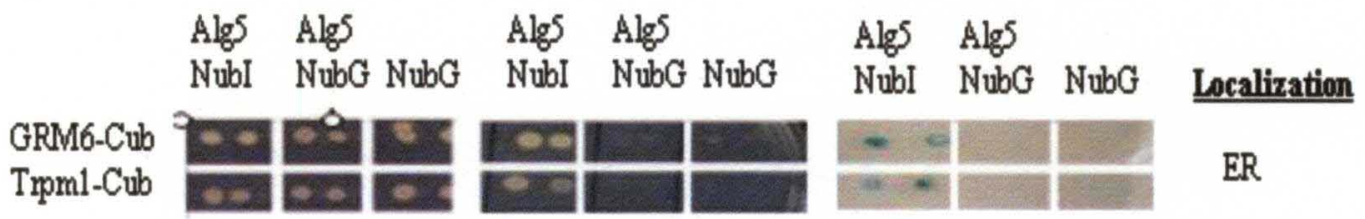

ii.

Fur4 Fur4 Fur4 Fur4 $\quad$ Fur4 Fur4

NubI NubG NubG NubI NubG NubG NubI NubG NubG

GRM6-Cub

Cub-Trpml

Tpml-Cub

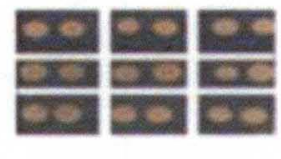

Capsazepine
B.

\section{EYFP-Trpm1 EYFP-Kir2.1}

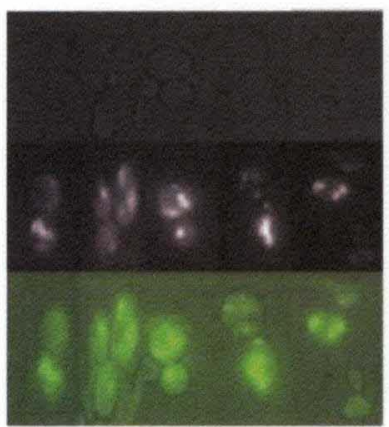


membrane. Co-transfection of either Trpm1-Lp1 or Trpm1-Lp3 with Fur4-NubI result in positive growth and $\beta$-galactosidase assay (Figure $28 \mathrm{C}$-iii). Together these data show that full length Trpml is not transported to the cell surface, which is consistent with the negative result in the cobalt assay. These data also suggest that nyctalopin alone cannot traffic the Trpml channel to the membrane in yeast cells.

\section{Identification of additional proteins that interact with nyctalopin}

To determine if other proteins interact with nyctalopin and might help to traffic the Trpm1 channel to the plasma membrane, I used the membrane split ubiquitin yeast two hybrid system. I screened my retinal yeast two hybrid cDNA library using nyctalopin (Nyc-Cub) as a bait. The retinal cDNA library consists of $1.397 \times 10^{6}$ primary clones with an average size of $1.38 \mathrm{~kb}$ (Table 3 ). Library construction and screening was described in the method section. The screen resulted in the growth of 36 yeast clones. The 36 clones represent 19 different genes. The prey plasmid from the clones was isolated, sequenced and the identity determined by Blast analysis (Table 4). In all cases the purified plasmids were retransformed into yeast with Nyc-Cub to confirm interaction. The confirmation assay indicated that B3gat3, Atpase6, Atp9a, MapK8ip1, Slc3a2, Smarca2 and Batla are false positives. These clones interacted non-specifically with other proteins (Alg5 and Fur4) beside nyctalopin. Frmd4a, Dpysl2, Fhod3, Ak040027, $R p l 3 a$, and Iqgap2 showed interaction with nyctalopin but the region interacting was not annotated as exons. Table 4, the region of interaction for these proteins is described as a potential exon. These clones are categorized as potential true positives because they might be splice variants of their respective proteins. Tbed13, Cecr2, and Pcbp2 are

cytoplasmic proteins. In chapter II, I determined that the entire leucine rich repeat of 
nyctalopin is in the extracellular space. How these proteins could be interacting with nyctalopin is not clear to me.

One of the proteins from the list is the amyloid precursor protein binding alpha one, Apba1. Two independent clones (Clone\# 25\&32) of different insert size identify Apbal as an interacting partner of nyctalopin. Isolation of the plasmids and retransformation with nyctalopin confirmed that the two clones are not false positives. Amyloid precursor protein binding alpha one (Apbal) is an adaptor protein involved in selective vesicular trafficking of proteins to postsynaptic density regions (Zhang et al., 2004; Guillaud et al., 2007). Localization studies of Apbal in insulin secreting $\beta$-cells show that Apbal is localized to the periphery of cells (Zhang et al., 2004). Three mammalian isoforms of Apba1 have been characterized (Okamoto et al., 1997; Okamoto et al., 1998; Tanahashi et al., 1999). Apbal is a multidomain protein consisting of an isoform specific $\mathrm{N}$-terminal, and a C-terminal PDZ and PTB binding domains (Duclos et al., 1995; Okamoto et al, 1997; Okamoto et al, 1998; Tanahashi et al., 1999). The PDZ domain has been shown to interact with kinesin 17 (Kif17), which is part of a molecular motor (Setou et al., 2000). The N-terminus binds to Munc-18 which is involved in vesicular exocytosis (Okamoto et al, 1997). Although my results are preliminary, it will be interesting to determine the ERG phenotype of Apbal knockout mice. If the ERG bwave is absent or significantly reduced in this mouse, it will indicate a potential involvement of Apba1 in the Grm6 signaling cascade or the localization of Trpm1 channel. Immunohistochemistry should be performed on mouse retinas to determine the localization of the Apba1. To determine if Trpm1 or nyctalopin affect the localization of Apba1, immunohistochemistry should be performed on retinas from $\mathrm{Trpm}^{-/ .}$and $\mathrm{Nyc}^{\text {nob }}$ 
mice. These results should confirm or reject my hypothesis that Apba1 is an interacting partner of nyctalopin and is involved in the Grm6 signaling cascade.

Overall these data suggest that a complex of proteins including nyctalopin, Apba1 and a kinesin family member are involved in the trafficking of the Trpm1 channel to the membrane.

\section{DISCUSSION}

Nyctalopin has been predicted to be involved in protein-protein interaction but no proteins have been identified as binding partners until now. In this chapter, I have provided evidence using membrane yeast two hybrid analyses and coimmunoprecipitation assays that nyctalopin can directly interact with the Trpm1 channel. These studies also indicate that the interaction domain in Trpml is the first and third extracellular domains, with the strongest interaction for extracellular loop 1.

What is the functional signification of the interaction between nyctalopin and Trpm1? Previous results from our lab suggest that in the absence of nyctalopin, Trpm1 is absent or not gated (Gregg et al., 2007). Localization studies using fluorescence microscopy and yeast two hybrid analysis with specifically targeted yeast membrane proteins shows that Trpml is not localized at the plasma membrane in yeast cells. In contrast nyctalopin is present at both the ER membrane and plasma membrane (Chapter II). Most of the Trp channels studied so far are also localized in intracellular vesicular membrane (Turner et al., 2003; Bezzerides et al., 2004; Krapvinsky et al., 2006; Dong et al., 2008). Recruitment of Trp channels to the plasma membrane is an alternative mechanism by which Trp channels are activated (Bezzerides et al., 2004; see review Montell, 2004). My data suggest that the interaction between nyctalopin and full length 
Trpm1 in yeast occurs at the ER membrane. Therefore, if nyctalopin alone is involved in the trafficking of Trpm1 to the plasma membrane, then co-expression of both proteins should result in their localization to the plasma membrane. This does not appear to be the case because in the presence of capsaicin and anandamide, there is no indication using the cobalt uptake assay that Trpm1 is on the cell surface. Unless the Trpm1 channel in yeast is not modulated by these pharmacological agents. These data suggest that nyctalopin alone does not affect the localization of the Trpm1 channel in yeast.

The Trpml channel is the non-selective cation channel in depolarizing bipolar cell (Shen et al., 2009; Morgan et al., 2009; Koike et al., 2009). How Trpm1 is gated or trafficked to the plasma membrane is not clear. Analysis of the gating properties of the Trpml channel in heterogeneous expression systems shows that the channel is constitutively open in vitro (Shen et al., 2009, Koike et al.2009). Because Trpm1 conducts $\mathrm{Ca}^{2+}$ this could lead to calcium overload, which could result in toxicity. The result could explain the lack of Trpml on the cell surface in my yeast experiments. Trpml has been shown to be blocked by capsazepine in depolarizing bipolar cell (Shen et al., 2009). Co-expression of Fur4-NubI and Cub-Trpm1 or Trpm1-Cub is negative in the presence or absence of capsazepine. These data suggest that the internal localization of Trpm1 is not because it is a constitutively open channel at the cell surface. It also suggests that yeast cells do not express Trpm1 channel at the plasma membrane in yeast ceslls. It might be that Trpm1 channel trafficking to the plasma membrane involves a complex of proteins. Several Trp channels are trafficked to the cell surface in this manner. Drosophila $\operatorname{Trp} / \operatorname{TrpL}$ and mammalian Trpc4/5 interact with the PDZ domain of InaD and NHERF-1 (Shieh et al., 
Table 2

\begin{tabular}{|c|c|c|c|}
\hline Library & Average Insert & Number of Primary & Expected \# of primary clones \\
& Size $(\mathrm{kb})$ & Clones & \\
\hline pDLS.Library & 1.38 & $1.397 \times 10^{6}$ & $1.0 \times 10^{6}$ \\
\hline
\end{tabular}

Summary of the complexity of the retinal cDNA library. 
Table 3. List of potential interacting partners for nyctalopin

\begin{tabular}{|c|c|c|c|c|c|}
\hline & Clone \# & Interactor & $\begin{array}{c}\text { Region } \\
\text { interacting }\end{array}$ & Comments & Status \\
\hline 1 & 8 & Frmd4a & possible exons & Isoform of Frmd4a & potential True \\
\hline 2 & 15 & Frmd4a & possible exons & Isoform of Frmd4a & potential True \\
\hline 3 & 23 & Frmd4a & possible exons & Isoform of Frmd4a & potential True \\
\hline 4 & 60 & Smarca2 & Exons & & potential false \\
\hline 5 & 19 & Mapk8ip 1 & Exons & & potential false \\
\hline 6 & 21 & Mapk8ip 1 & Exons & & potential false \\
\hline 7 & 50 & pcbp2 & Exons & & \\
\hline 8 & 29 & Iqgap2 & possible exons & Isoform of Iqgap2 & \\
\hline 9 & 47 & Iqgap2 & possible exons & Isoform of Iqgap 2 & \\
\hline 10 & 44 & Tbcld13 & Exons & & \\
\hline 11 & 36 & B3gat3 & Exons & & potential \\
\hline 12 & 4 & Atpase6 & Exons & & potential True \\
\hline 13 & 45 & Atp9a & Exons & & potential false \\
\hline 14 & 66 & Atp9a & Exons & & potential false \\
\hline 15 & 57 & Hnt & & & \\
\hline 16 & 30 & Cecr $2 / A k 48664$ & Exons & Not much is known & potential \\
\hline 17 & 75 & Cecr $2 / A k 48664$ & Exons & Not much is known & potential \\
\hline 18 & 7 & Dpys12 & Exons/5UTR & Axon/soma & potential \\
\hline 19 & 34 & Dpysl2 & Exons/5UTR & Axon/soma & potential \\
\hline 20 & 25 & Apba1 & Exons & Trafficking to PSD & potential \\
\hline 21 & 32 & Apbal & Exons & Trafficking to PSD & potential \\
\hline 22 & 41 & Gse 1 & Exons & Not much is known & potential \\
\hline 24 & 18 & Slc $3 \mathrm{a} 2$ & Exons & Integral to membrane & potential \\
\hline 25 & 77 & Slc $3 \mathrm{a} 2$ & Exons & Integral to membrane & potential \\
\hline 26 & 80 & Slc $3 \mathrm{a} 2$ & Exons & Integral to membrane & potential \\
\hline 27 & 74 & Bat1a & Exons & & potential false \\
\hline 28 & 48 & Rpl13a or Snords & possible exons & & \\
\hline 29 & 67 & Rpl13a or Snords & possible exons & & \\
\hline 30 & 59 & Ak040027 & possible gene & EST & potential \\
\hline 31 & 26 & Fhod3 & possible exons & & potential True \\
\hline 32 & 40 & Fhod3 & possible exons & & potential True \\
\hline 33 & 42 & Fhod3 & possible exons & & potential True \\
\hline 34 & 65 & Fhod3 & possible exons & & potential True \\
\hline 35 & 76 & Fhod3 & possible exons & & potential True \\
\hline
\end{tabular}

\# denote number 
1996; Tang et al., 2000; Obukhov et al., 2004). A point mutation in the PDZ domain of InaD disrupts the interaction between InaD and Trp, which result in the mislocalization of Trp to the cells bodies (Chevesich et al., 1997). The amount of Trpc4 at the plasma membrane is decreased because of the loss of interaction between Trpc 4 and NHERF-1 (Mery et al., 2002). Trafficking of the Trp channel to the cell surface involves vesicle loading, budding, targeting, docking, fusion and motility (Zerial et al., 2001; Smythe et al., 2002). Most of these steps are modulated by small G proteins (Van de Graaf et al., 2006), adapter proteins, and the kinesin family of proteins (Setou et al., 2000; Guillaud et al., 2007). In my yeast two hybrid screen I found that nyctalopin interacts with Apbal. Apbal is an adapter protein involved in vesicular trafficking in neurons. Three mammalian isoforms of Apbal have been characterized (Okamoto et al., 1997; Okamoto et al., 1998; Tanahashi et al., 1999). They are composed of an isoform specific Nterminal domain, and a common C-terminal PTB and PDZ domains. The N-terminal domain allows isoform specific interaction. The region that interacted with nyctalopin is part PTD and part N-terminal domain. Localization studies of Apbal in insulin secreting $\beta$-cells show that Apbal is localized to the periphery of cells (Zhang et al., 2004). In vertebrates and Caenorhabditis elegans, Apba1 is localized to the Golgi apparatus as well as the axons and dendrites of neurons (Whitfield et al., 1999; Biederer et al., 2002). Apbal has also been shown to directly interact with Kinesin 17 which is part of a molecular motor in neurons.

So I am proposing that a complex of proteins including nyctalopin and Apbal is involved in vesicular trafficking of the Trpm1 channel to the cell surface in depolarizing bipolar cell. 


\section{CHAPTER IV}

\section{CONCLUSIONS}

Congenital stationary night blindness (CSNB) is a group of genetic retinal disorders that are characterized by impaired night vision of different degrees (Cunier et al., 1838). In some human patients this disease is caused by mutations in the $N y x$ gene, which encodes the protein nyctalopin (Bech-Hansen et al., 2000; Pusch et al., 2000). To date, 35 mutations have been identified in human patients (Bech-Hansen et al., 2000; Pusch et al., 2000, Jacobi et al., 2002). CSNB1 is diagnosed by the absence of a b-wave in the electroretinogram (ERG) (Miyake et al., 1986). Mutations in Grm6, G $\alpha \mathrm{O} 1, \mathrm{G} \beta 5$ and Trpm1 also have been found to cause CSNB1 (Masu et al., 1995; Pardue et al., 1998; Dhingra et al., 2000; Bech-Hansen et al., 2000; Pusch et al., 2000; Gregg et al., 2003; Dryja et al., 2005; Pinto et al., 2007; Maddox et al., 2008; Bellone et al., 2008; Shen et al., 2009; Aldo et al., 2009; Van Genderen et al., 2009; Li et al., 2009) indicating that all of these proteins are involved in the same signaling cascade.

In the dark, there is a tonic release of glutamate by the photoreceptors into the OPL. When the glutamate binds to the metabotropic glutamate receptor (GRM6), it activates a Gprotein signal transduction cascade that closes a non-specific cation channel now known to be Trpml (Kim et al., 2008; Bellone et al., 2008; Shen et al., 2009; Morgan et al., 2009; Koike et al., 2009). When there is an increase in light intensity, the photoreceptors 
hyperpolarize, and glutamate release is decreased. These results in reduced Grm6 activity and the opening of the Trpm1 channel. The opening of the Trpm1 channel causes the depolarization of the depolarizing bipolar cell, which results in the b-wave seen in ERG.

The no b-wave mouse, $N y x^{n o b}$, is a model for CSNB1 (Pardue et al., 1998; Gregg et al., 2003). The mutation in $N y x^{n o b}$ is an $85 \mathrm{bp}$ deletion in exon 3 of the $N y x$ gene that is predicted to cause a frame shift and truncation of nyctalopin (Gregg et al., 2003). Nyctalopin is a member of the small leucine rich proteoglygan (SLRP) protein family. At the $\mathrm{N}$-terminus, it consists of eleven leucine rich repeats (LRR) which are capped at the Nand C-terminus by cysteine rich LRRs.

The exact role of nyctalopin is not known. In chapter II, I have shown that nyctalopin's entire leucine rich domain is in the extracellular space. This confirms the immunofluorence data that suggest that nyctalopin is a cell surface protein (Zeitz et al., 2003; Gregg et al., 2007). I have also shown that murine nyctalopin is anchored to the membrane by a single transmembrane domain. These data confirm the prediction by Gregg et al., (2003) and O'Conner et al. (2005) that murine nyctalopin is not GPI anchored to the membrane (Gregg et al., 2003; O'Conner et al. 2005). The difference in anchoring might not result in functional differences between the two orthologues. The transmembrane domain of murine nyctalopin is located between residues $353-472$ which means that only four amino acids are intracellular.

How is nyctalopin which is entirely extracellular, involved in modulating the intracellular Grm6 signaling cascade? Previous data from our lab determined that in the absence of nyctalopin the Trpm1 channel is absent or not gated (Gregg et al., 2007). Based 
on topology, nyctalopin is involved in assembling and/or trafficking of the Trpm1 channel to the plasma membrane.

In chapter III, I have shown that nyctalopin directly interacts with the extracellular loops 1 and 3 of Trpm1. This interaction occurs in both yeast two hybrid and in vitro translation systems. These data provide evidence that nyctalopin might be involved in regulating some function of Trpm1. Trpml has been shown to be the nonselective cation channel in depolarizing bipolar cells (Shen et al., 2009, Morgans et al., 2009; Koike et al., 2009) but how this channel is gated in depolarizing bipolar cells is yet to be determined. Trpm1 staining in depolarizing bipolar cells is present at both the cell bodies and the dendritic tips (Morgans et al., 2009; Koike et al., 2009). Localization studies in yeast show that Trpml is not localized to the plasma membrane (Chapter III). Heterogeneous expression systems show that the Trpm1 channel is mainly intracellular and that when localized at the plasma membrane, it is a constitutively open channel (Xu et al., 2001; Oancea et al. 2009; Koike et al., 2009). A constitutive open channel at the plasma membrane will cause $\mathrm{Ca}^{2+}$ overload, which could be toxic to the cells. From my data it is possible there is a three way interaction between nyctalopin, Trpm1 and Apba1, which would suggest that a complex of proteins is involved in either the assembling or trafficking of the Trpm1 channel to the plasma membrane (Chapter III). The other scenario could be that the interaction is between nyctalopin, Trpml and VWF (Appendix). VWF domain containing proteins were shown to be involved in the trafficking of voltage dependent calcium channels to the plasma membrane (Canti et al., 2005). Although I have not determined interaction between VWF and nyctalopin or 
Trpm1, VWF has been shown to interact with decorin (Guidetti et al., 2004). Decorin is in the same SLRP family as nyctalopin.

There is no common mechanism by which Trp channels are gated. In terms of activation through G-protein coupled receptors, three models have been proposed. The activation of G-protein couple receptors (GPCR) results in the exchange of GDP for GTP on the $\mathrm{G} \alpha$ subunit of a trimeric $G \alpha \beta \gamma$ subunit. This results in the release of the trimeric $\mathrm{G} \alpha \beta \gamma$ from the GPCR allowing it to dissociate into $\mathrm{Ga}^{\mathrm{GTP}}$ and $\mathrm{G} \beta \gamma$ subunits. Either $\mathrm{G} \alpha^{\mathrm{GTP}}$ or $\mathrm{G} \beta \gamma$ can then go on to activate various signaling molecules, which in turn can gate the Trp channel.

In the first model, activation is through a diffusible messenger called the calcium influx factor (CIF). Phospholipase $\mathrm{C}$ is activated by either $\mathrm{G \alpha}^{\mathrm{GTP}}$ or G $\beta \gamma$. Activated phospholipase $\mathrm{C}$ then catalyzes the hydrolysis of phospho-inositol-bisphosphate $\left(\mathrm{PIP}_{2}\right)$ into diacylglycerol (DAG) and inositol trisphosphate $\left(\mathrm{IP}_{3}\right) . \mathrm{IP}_{3}$ activates the $\mathrm{IP}_{3}$ receptor $\left(\mathrm{IP}_{3} \mathrm{R}\right)$ at the ER membrane to release calcium from the internal calcium stores. $\mathrm{Ca}^{2+}$ as a second messenger then closes the Trp channel at the plasma membrane (Figure 27). When internal calcium stores are depleted, the calcium influx factor is released and it stimulates calcium influx into the cells (Randriamampita et al., 2005) through Trp channels at the plasma membrane (Figure 27). The molecular identity of the calcium influx factor is not known (See review by Parekh et al., 2005; Bolotina et al., 2005). The other problem with this model is that Trp channels are not selective to just $\mathrm{Ca}^{2+}$. Drosophila Trp/Trpl and mammalian Trpvl have been shown to be inhibited by $\mathrm{PIP}_{2}$ (Chuang et al., 2001; Hardie et al., 2003), however, Trp channels are not activated by thapsigargin (blocks store operated channel in ER membrane), $\mathrm{Ca}^{2+}$ or $\mathrm{IP}_{3}$ (Ranganathan 
et al., 1994; Hardie, 1995, 1996; Hardie and Raghu, 1998). In addition $\mathrm{IP}_{3} \mathrm{R}$ mutants are completely normal with respect to gating the Trp channels (Acharya et al., 1997; Raghu et al., 2000). These data suggest that gating the Trp channel through internal calcium stores at the ER membrane is not a plausible one. In addition, it also suggests that calcium as a second messenger does not gate the Trp channel. However there is evidence for coupling between Trp channels and inositol trisphosphate receptors $\left(\mathrm{IP}_{3} \mathrm{R}\right)$ (Berridge et al., 1990; Irvine et al., 1990). In endothelial cells thrombin stimulation results in the translocation of Trpc1-IP3R complex to the plasma membrane (Mehta et al., 2003). This translocation is dependent on the RhoA, a small GTP-binding protein (Ridley et al., 2001).

In the second model either $\mathrm{G} \alpha^{\mathrm{GTP}}$ or $\mathrm{G} \beta \gamma$ can activate phospholipase C (PLC), which catalyze the hydrolysis of phospho-inositol-bisphosphate $\left(\mathrm{PIP}_{2}\right)$ into diacylglycerol (DAG) and inositol trisphosphate $\left(\mathrm{IP}_{3}\right)$. DAG through an unknown mechanism can lead to the closure of Trp channels at the plasma membrane (Figure 28). Mutation of the gene encoding diacyglycerol kinase (DAGK) has been shown to cause constitutive activation of the Trp and Trpl channel in Drosophila (Raghu et al., 2000b). A single stimulation of the GPCR results in the release of millions of $\mathrm{IP}_{3}$ or DAG molecules, which makes this cascade extremely fast. In addition this model encompasses most of the molecules involved in Grm6 signaling cascade. In this model, nyctalopin could be functioning to assemble the homotetameric Trpm1 channel at the ER or Golgi membrane. If this model is the correct model for signaling in depolarizing bipolar cell, then the Trpml channel cannot be constitutively open. The constitutive activity of Trp channels in vitro (Oancea et al., 2009; Koike et al., 2009) is a result of over expressing Trp channels in non-native 
Figure 27. Trp channel gating through Calcium Influx factor (CIF). In the dark, glutamate binding to the Grm6 receptors result in the exchange of GDP for GTP. The exchange of GTP for GDP can be accelarated by the GEF activity of PCP2. The trimeric G-protein is release from the Grm6 receptor and it dissociate into $\mathrm{G} \alpha$ and $\mathrm{G} \beta \gamma$. Either $\mathrm{G} \alpha$ or G $\beta \gamma$ can activate phospholipase $\mathrm{C}$ which catalyzes the hydrolysis of phosphor-inositolbisphosphate $\left(\mathrm{PIP}_{2}\right)$ into diacylglycerol $(\mathrm{DAG})$ and inositol trisphosphate $\left(\mathrm{IP}_{3}\right) . \mathrm{IP}_{3}$ activates $\mathrm{IP}_{3}$ receptor at the ER membrane to release $\mathrm{Ca}^{2+}$. As a second messenger $\mathrm{Ca} 2+$ through an unknown mechanism closes the Trpm1 channel at the membrane. During light activation, the signal from the Grm6 receptor is inactivated. The internal $\mathrm{Ca}^{2+}$ stores are depleted, which stimulates the release of the calcium influx factor (CIF). CIF stimulate $\mathrm{Ca}^{2+}$ influx through Trpm1 channel at the membrane. 

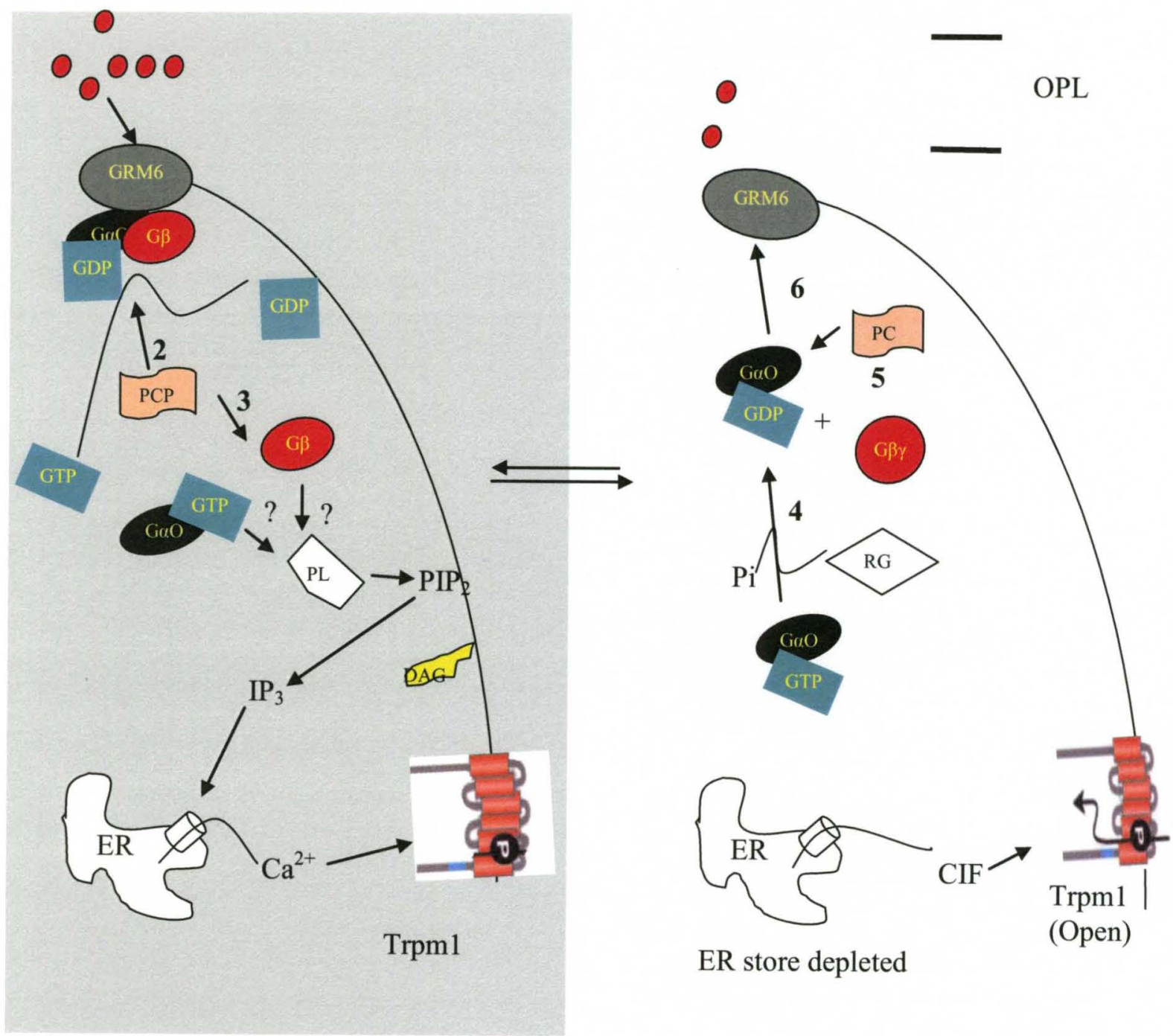

ER store depleted 
conditions. The trafficking of the nyctalopin-Trpm 1 complex to the plasma membrane is a constitutive process in this model. Signaling from the Grm6 receptor does not affect the trafficking of the Trpm1 channel to the plasma membrane. Trafficking is a cells way for cells to regulate the level of surface proteins in the plasma membrane.

Assuming this model is correct, the following events could occur: Grm6 receptor activation resulting in the exchange of GDP for GTP on the GaO subunit. The trimeric $\mathrm{G} \alpha \beta \gamma$ is released from the Grm6 receptor and either $\mathrm{G} \alpha^{\mathrm{GTP}}$ or $\mathrm{G} \beta \gamma$ can activate phospholipase C (PLC). PLC then catalyzes the hydrolysis of PIP2 into DAG and IP3. DAG through an unknown mechanism then goes on to close the Trpm1 channel. However if nyctalopin, which assembles the Trpml channel at the ER or the Golgi is lost, the Trpml channel disassembles and is not trafficked to the plasma membrane (Figure 28).

The third model involves the dynamic insertion of the Trp channels into the plasma membrane (Figure 29). The Trp channels are held in sub-plasmalemmal vesicle pools (Yao et al., 1999) and activation causes their insertion into the membrane. In this model Trp channels are constitutively open therefore their trafficking to and from the plasma membrane are regulated. There is a lot of evidence for this kind of gating for Trp channel in the literature.

Studies show that a non-hydrolyzable anologue of GTP has an inhibitory effect on $\mathrm{Ca}^{2+}$ entry through Trp channels (Bird et al., 1993; Fasolato et al., 1993; Somasundaram et al., 1995; Fernando et al., 1997). These studies implicate small GTP binding proteins, which are involved in vesicular trafficking, in Trp channel gating. In DDT1MF-2 and 
Figure 28. Trpm1 gating through Diacylglycerol (DAG). In the dark, glutamate binding to the Grm6 receptor results in the exchange of GDP for GTP (step 1). The exchange of GTP for GDP can be accelarated by the GEF activity of PCP2 (step 2). The trimeric G-protein is released from the Grm6 receptor and dissociates into G $\alpha^{\mathrm{GTP}}$ and $\mathrm{G} \beta \gamma$. Either $\mathrm{G} \alpha^{\mathrm{GTP}}$ or $\mathrm{G} \beta \gamma$ can activate phospholipase $\mathrm{C}$ which catalyzes the hydrolysis of phospho-inositol-bisphosphate $\left(\mathrm{PIP}_{2}\right)$ into diacylglycerol (DAG) and inositol trisphosphate $\left(\mathrm{IP}_{3}\right)$. DAG through an unknown mechanism then goes on to close the Trpm1 channel. In the dark, glutamate release is reduced. The GTP on the G $\alpha^{\text {GTP }}$ subunit is hydrolyzed by RGS protein (RGS11 or RGS7). Ga ${ }^{\mathrm{GDP}}$ then binds the G $\beta \gamma$ subunit and the trimeric G-protein subunit coupled back to the Grm6 receptor. Through GDI activity, PCP2 can accelerate the coupling of the trimeric G-protein to the Grm6 receptor by binding to the $\mathrm{G} \alpha^{\mathrm{GDP}}$ subunit. This reduces the amount of $\mathrm{G} \alpha$ available to activate PLC. In this model there is a constitutive trafficking of Trpml channel to the plasma membrane. Nyctalopin is involved in the assembling of the Trpml channels at the ER membrane before it is trafficked to the plasma membrane. 


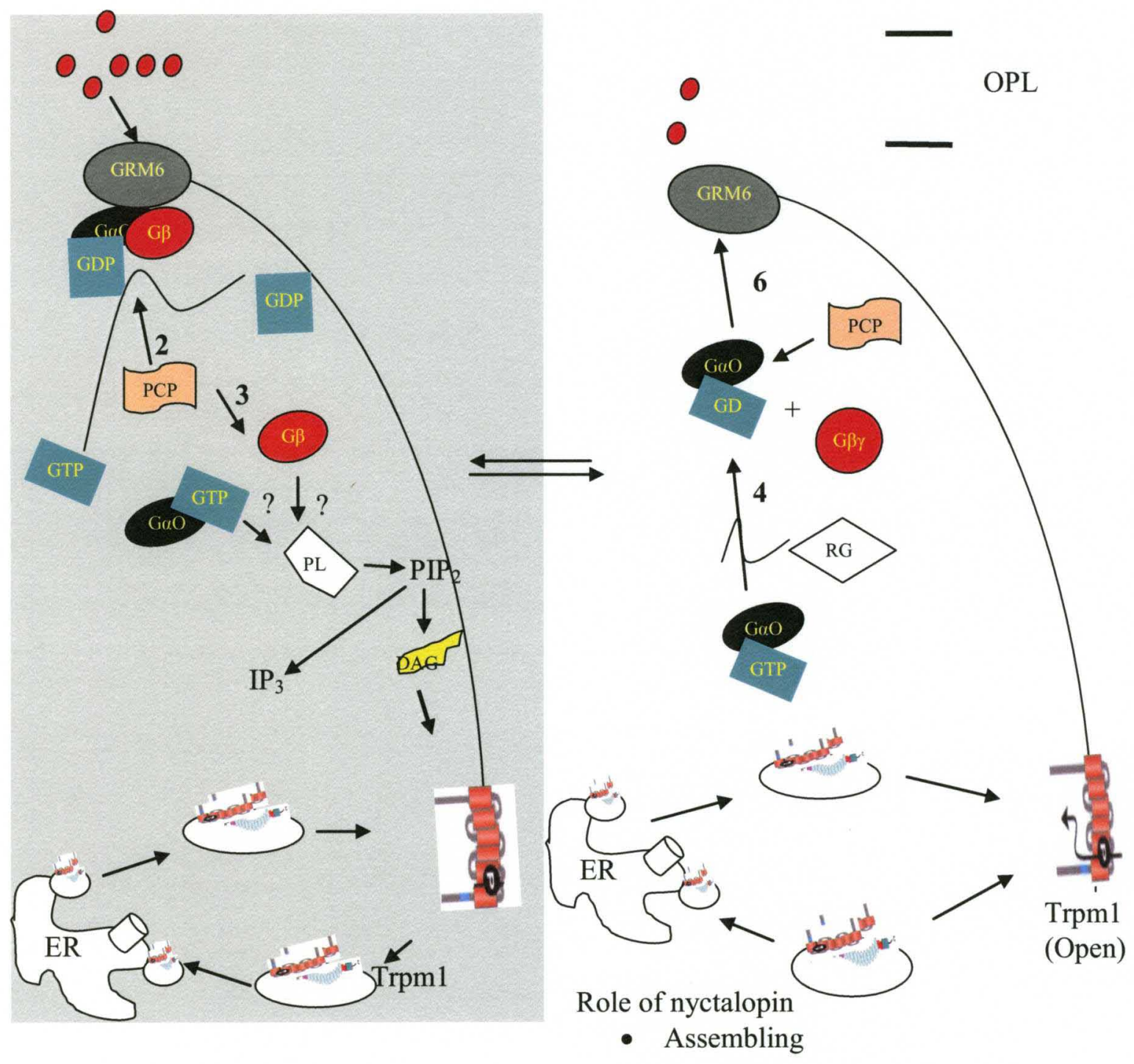


Figure 29. Trafficking as a way of gating Trpm1 channel from plamalemmal visicle pool. In the dark, glutamate binding to the Grm6 receptors result in the exchange of GDP for GTP (step 1). The exchange of GTP for GDP can be accelarated by the GEF activity of PCP2 (step 2). The trimeric G-protein is release from the Grm6 receptor and it dissociates into $\mathrm{G} \alpha^{\mathrm{GTP}}$ and $\mathrm{G} \beta \gamma$. Either $\mathrm{G} \alpha^{\mathrm{GTP}}$ or $\mathrm{G} \beta \gamma$ can cause the Trpml channel at the membrane to be internalized or inhibit the trafficking of the Trpm1 channel to the plasma membrane. Trpm1 is a constitutively open channel and its gating is regulated by its trafficking. During light increment, the signal that causes the Trpm1 to internalize or that inhibits them from trafficking to the plasma membrane is removed by inactivation of the Grm6 receptor. Vesicles held in plasmalemmal vesicle pools are quickly trafficked to the plasma membrane, which results in rapid $\mathrm{Ca}^{2+}$ and $\mathrm{Na}^{+}$influx into the depolarizing bipolar cell. Nyctalopin is involved in the assembling of the Trpm1 channel. 


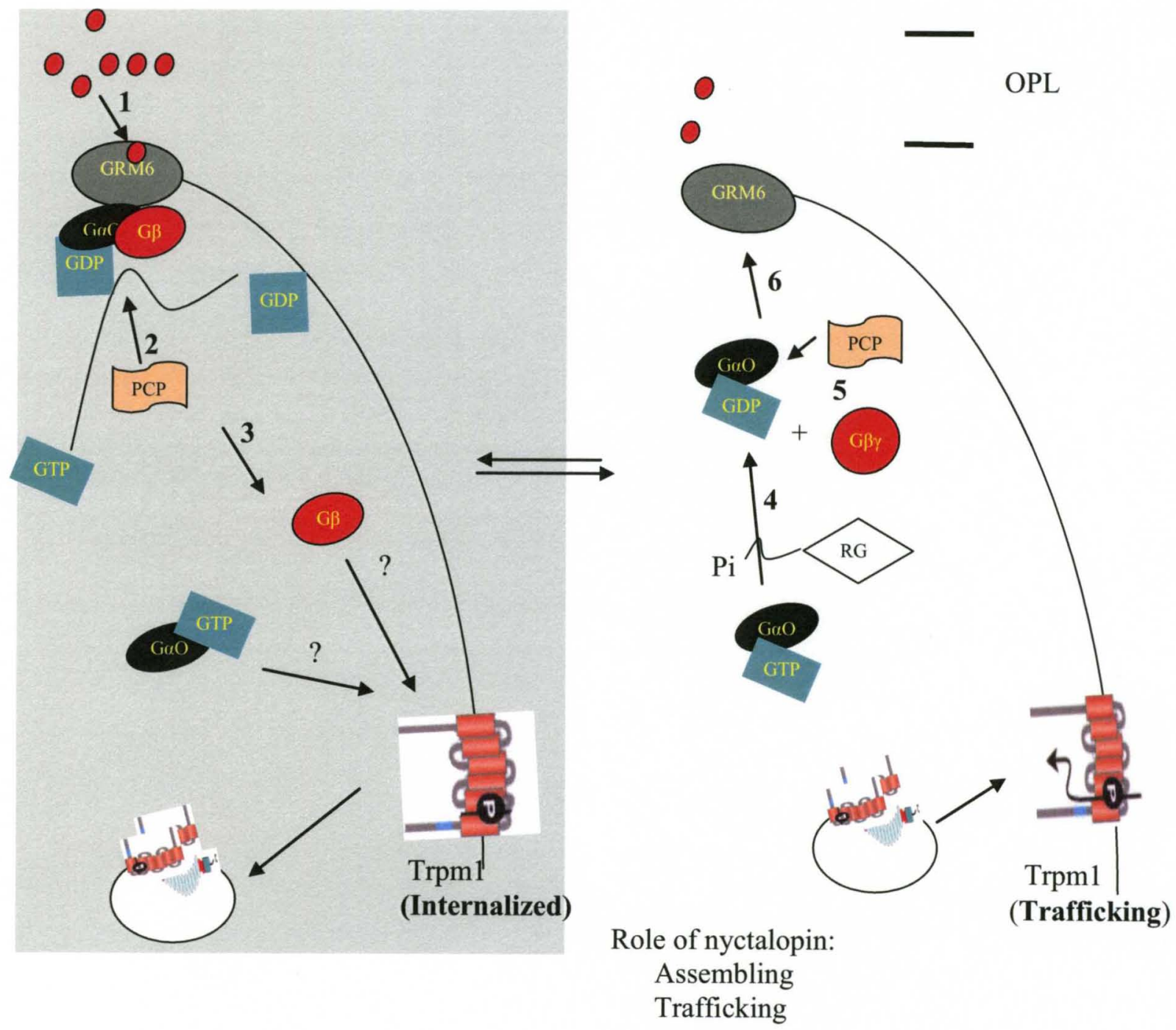


A7r 5 cells, $\mathrm{Ca}^{2+}$ entry through Trp channels is abrogated in the presence of cytoskeletonreorganizing drugs (Patterson et al., 1999). In vitro studies indicate that cytoskeletal reorganization plays a key role in exocytosis and endocytosis of several Trp channels (Itagaki et al., 2004; Mehta et al., 2003; Lockwich et al., 2001; Cayouette et al., 2004). In fact in vitro translocation of Trpv5/6 requires interaction with the S100A10-annexin-2 complex, which is associated with the cortical cytoskeleton (Van de Graaf et al., 2003). Activation of the receptor tyrosine pathway results in the dynamic insertion of the Trpc5 into the plasma membrane (Bezzerides et al., 2004). Trpc3 translocates from intracellular vesicle to the plasma membrane during sperm activation (Xu et al., 2003). In the dark Drosophila Trpl translocates from the intracellular compartments to the rhabdomeral membrane and during light stimulation Trpl channel is internalized to the intracellular compartments (Bahner et al., 2002; Meyer et al., 2006; Cronin et al., 2006). These data provide the first in vivo model for Trp channel trafficking as a way of gating.

Although activation of the Grm6 signaling cascade closes the Trpml channel, it is possible that the signal from the Grm6 receptor prevents the trafficking of the Trpml to the membrane or causes the Trpm1 channel at the membrane to internalize (Figure 29). In the dark, Trpm1 channels are localized to intracellular sub-plasmalemmal vesicle pool. When light intensity increases the Trpml channel at the sub-plasmalemmal vesicle pool translocate to the plasma membrane causing influx of $\mathrm{Ca}^{2+}$ and $\mathrm{Na}^{+}$ions. In this case nyctalopin will be involved in the assembling of the Trpml channel to the membrane. Without nyctalopin, the complex disassembles and the Trpm1 channel cannot be trafficked to the plasma membrane. 


\section{REFERENCES}

Acharya, J.K., Jalink, K., Hardy, R.W., Hartenstein, V., and Zuker, C.S. (1997). InsP3 receptor is essential for growth and differentiation but not for vision in Drosophila. Neuron 18, 881-887.

Arai, M., Mitsuke, H., Ikeda, M., Xia, J.X., Kikuchi, T., Satake, M., and Shimizu, T. (2004). ConPred II: a consensus prediction method for obtaining transmembrane topology models with high reliability. Nucleic Acids Res 32, W390-393.

Attwell, D., Tessier-Lavigne, M., Wilson, M., and Mobbs, P. (1987). Bipolar cell membrane currents and signal processing in the axolotl retina. Neurosci Res Suppl 6, S191-204.

Auerbach, E., Godel, V., and Rowe, H. (1969). An electrophysiological and psychophysical study of two forms of congenital night blindness. Invest Ophthalmol 8 , 332-345.

Baas, D., Bumsted, K.M., Martinez, J.A., Vaccarino, F.M., Wikler, K.C., and Barnstable, C.J. (2000). The subcellular localization of Otx2 is cell-type specific and developmentally regulated in the mouse retina. Brain Res Mol Brain Res 78, 26-37.

Bahner, M., Frechter, S., Da Silva, N., Minke, B., Paulsen, R., and Huber, A. (2002). Light-regulated subcellular translocation of Drosophila TRPL channels induces long-term adaptation and modifies the light-induced current. Neuron 34,83-93.

Balkema, G.W. (1991). A synaptic antigen (B16) is localized in retinal synaptic ribbons. J Comp Neurol 312, 573-583.

Ball, S.L., and Gregg, R.G. (2002). Using mutant mice to study the role of voltage-gated calcium channels in the retina. Adv Exp Med Biol 514, 439-450.

Ball, S.L., Pardue, M.T., McCall, M.A., Gregg, R.G., and Peachey, N.S. (2003). Immunohistochemical analysis of the outer plexiform layer in the nob mouse shows no abnormalities. Vis Neurosci 20, 267-272.

Ball, S.L., Powers, P.A., Shin, H.S., Morgans, C.W., Peachey, N.S., and Gregg, R.G. (2002). Role of the beta(2) subunit of voltage-dependent calcium channels in the retinal outer plexiform layer. Invest Ophthalmol Vis Sci 43, 1595-1603. 
Bansal, A., Singer, J.H., Hwang, B.J., Xu, W., Beaudet, A., and Feller, M.B. (2000). Mice lacking specific nicotinic acetylcholine receptor subunits exhibit dramatically altered spontaneous activity patterns and reveal a limited role for retinal waves in forming ON and OFF circuits in the inner retina. J Neurosci 20, 7672-7681.

Bech-Hansen, N.T., Boycott, K.M., Gratton, K.J., Ross, D.A., Field, L.L., and Pearce, W.G. (1998a). Localization of a gene for incomplete X-linked congenital stationary night blindness to the interval between DXS6849 and DXS8023 in Xp11.23. Hum Genet 103, 124-130.

Bech-Hansen, N.T., Naylor, M.J., Maybaum, T.A., Pearce, W.G., Koop, B., Fishman, G.A., Mets, M., Musarella, M.A., and Boycott, K.M. (1998b). Loss-of-function mutations in a calcium-channel alphal-subunit gene in Xp11.23 cause incomplete X-linked congenital stationary night blindness. Nat Genet 19, 264-267.

Bech-Hansen, N.T., Naylor, M.J., Maybaum, T.A., Sparkes, R.L., Koop, B., Birch, D.G., Bergen, A.A., Prinsen, C.F., Polomeno, R.C., Gal, A., et al. (2000). Mutations in NYX, encoding the leucine-rich proteoglycan nyctalopin, cause $\mathrm{X}$-linked complete congenital stationary night blindness. Nat Genet 26, 319-323.

Bellone, R.R., Brooks, S.A., Sandmeyer, L., Murphy, B.A., Forsyth, G., Archer, S., Bailey, E., and Grahn, B. (2008). Differential gene expression of TRPM1, the potential cause of congenital stationary night blindness and coat spotting patterns (LP) in the Appaloosa horse (Equus caballus). Genetics 179, 1861-1870.

Berrebi, A.S., Oberdick, J., Sangameswaran, L., Christakos, S., Morgan, J.I., and Mugnaini, E. (1991). Cerebellar Purkinje cell markers are expressed in retinal bipolar neurons. J Comp Neurol 308, 630-649.

Berridge, M.J. (1990). Temporal aspects of calcium signalling. Adv Second Messenger Phosphoprotein Res 24, 108-114.

Bezzerides, V.J., Ramsey, I.S., Kotecha, S., Greka, A., and Clapham, D.E. (2004). Rapid vesicular translocation and insertion of TRP channels. Nat Cell Biol 6, 709-720.

Biederer, T., Cao, X., Sudhof, T.C., and Liu, X. (2002). Regulation of APP-dependent transcription complexes by Mint/X11s: differential functions of Mint isoforms. $J$ Neurosci 22, 7340-7351.

Biel, M., Zong, X., and Hofmann, F. (1999). Cyclic nucleotide gated channels. Adv Second Messenger Phosphoprotein Res 33, 231-250.

Bird, G.S., and Putney, J.W., Jr. (1993). Inhibition of thapsigargin-induced calcium entry by microinjected guanine nucleotide analogues. Evidence for the involvement of a small G-protein in capacitative calcium entry. J Biol Chem 268, 21486-21488. 
Blackshaw, S., Harpavat, S., Trimarchi, J., Cai, L., Huang, H., Kuo, W.P., Weber, G., Lee, K., Fraioli, R.E., Cho, S.H., et al. (2004). Genomic analysis of mouse retinal development. PLoS Biol 2, E247.

Blankenship, A.G., and Feller, M.B. (2010). Mechanisms underlying spontaneous patterned activity in developing neural circuits. Nat Rev Neurosci 11, 18-29.

Blankenship, A.G., Ford, K.J., Johnson, J., Seal, R.P., Edwards, R.H., Copenhagen, D.R., and Feller, M.B. (2009). Synaptic and extrasynaptic factors governing glutamatergic retinal waves. Neuron $62,230-241$.

Blanks, J.C., Adinolfi, A.M., and Lolley, R.N. (1974). Synaptogenesis in the photoreceptor terminal of the mouse retina. J Comp Neurol 156, 81-93.

Blobel, G. (1971). Release, identification, and isolation of messenger RNA from mammalian ribosomes. Proc Natl Acad Sci U S A 68, 832-835.

Bobanovic, L.K., Royle, S.J., and Murrell-Lagnado, R.D. (2002). P2X receptor trafficking in neurons is subunit specific. J Neurosci 22, 4814-4824.

Bolotina, V.M., and Csutora, P. (2005). CIF and other mysteries of the store-operated Ca2+-entry pathway. Trends Biochem Sci 30, 378-387.

Boos, R., Schneider, H., and Wassle, H. (1993). Voltage- and transmitter-gated currents of all-amacrine cells in a slice preparation of the rat retina. J Neurosci 13, 2874-2888.

Borodinsky, L.N., Root, C.M., Cronin, J.A., Sann, S.B., Gu, X., and Spitzer, N.C. (2004). Activity-dependent homeostatic specification of transmitter expression in embryonic neurons. Nature 429, 523-530.

Boycott, B.B., and Wassle, H. (1991). Morphological Classification of Bipolar Cells of the Primate Retina. Eur J Neurosci 3, 1069-1088.

Brandstatter, J.H., Fletcher, E.L., Garner, C.C., Gundelfinger, E.D., and Wassle, H. (1999). Differential expression of the presynaptic cytomatrix protein bassoon among ribbon synapses in the mammalian retina. Eur J Neurosci 11, 3683-3693.

Brandstatter, J.H., Koulen, P., and Wassle, H. (1997). Selective synaptic distribution of kainate receptor subunits in the two plexiform layers of the rat retina. J Neurosci 17 , 9298-9307.

Brandstatter, J.H., Lohrke, S., Morgans, C.W., and Wassle, H. (1996a). Distributions of two homologous synaptic vesicle proteins, synaptoporin and synaptophysin, in the mammalian retina. J Comp Neurol 370, 1-10. 
Brandstatter, J.H., Wassle, H., Betz, H., and Morgans, C.W. (1996b). The plasma membrane protein SNAP-25, but not syntaxin, is present at photoreceptor and bipolar cell synapses in the rat retina. Eur J Neurosci 8, 823-828.

Brown, K.T. (1965). An early potential evoked by light from the pigment epitheliumchoroid complex of the eye of the toad. Nature 207, 1249-1253.

Buckley, K.M., Melikian, H.E., Provoda, C.J., and Waring, M.T. (2000). Regulation of neuronal function by protein trafficking: a role for the endosomal pathway. J Physiol 525 Pt 1, 11-19.

Calvert, P.D., Strissel, K.J., Schiesser, W.E., Pugh, E.N., Jr., and Arshavsky, V.Y. (2006). Light-driven translocation of signaling proteins in vertebrate photoreceptors. Trends Cell Biol 16, 560-568.

Canti, C., Nieto-Rostro, M., Foucault, I., Heblich, F., Wratten, J., Richards, M.W., Hendrich, J., Douglas, L., Page, K.M., Davies, A., et al. (2005). The metal-ion-dependent adhesion site in the Von Willebrand factor-A domain of alpha2delta subunits is key to trafficking voltage-gated Ca2+ channels. Proc Natl Acad Sci U S A 102, 11230-11235.

Cao, Y., Song, H., Okawa, H., Sampath, A.P., Sokolov, M., and Martemyanov, K.A. (2008). Targeting of RGS7/Gbeta5 to the dendritic tips of ON-bipolar cells is independent of its association with membrane anchor R7BP. J Neurosci 28, 1044310449.

Caras, I.W., Weddell, G.N., and Williams, S.R. (1989). Analysis of the signal for attachment of a glycophospholipid membrane anchor. J Cell Biol 108, 1387-1396.

Carr, R.E. (1974). Congenital stationary nightblindness. Trans Am Ophthalmol Soc 72, 448-487.

Cartin, L., Lounsbury, K.M., and Nelson, M.T. (2000). Coupling of $\mathrm{Ca}(2+)$ to CREB activation and gene expression in intact cerebral arteries from mouse : roles of ryanodine receptors and voltage-dependent $\mathrm{Ca}(2+)$ channels. Circ Res 86, 760-767.

Cayouette, S., and Boulay, G. (2007). Intracellular trafficking of TRP channels. Cell Calcium 42, 225-232.

Chang, B., Heckenlively, J.R., Bayley, P.R., Brecha, N.C., Davisson, M.T., Hawes, N.L., Hirano, A.A., Hurd, R.E., Ikeda, A., Johnson, B.A., et al. (2006). The nob2 mouse, a null mutation in Cacnalf: anatomical and functional abnormalities in the outer retina and their consequences on ganglion cell visual responses. Vis Neurosci 23, 11-24.

Chapman, B., and Godecke, I. (2000). Cortical cell orientation selectivity fails to develop in the absence of ON-center retinal ganglion cell activity. J Neurosci 20, 1922-1930. 
Chen, F.S., Shim, H., Morhardt, D., Dallman, R., Krahn, E., McWhinney, L., Rao, A., Gold, S.J., and Chen, C.K. (2010). Functional redundancy of R7 RGS proteins in ONbipolar cell dendrites. Invest Ophthalmol Vis Sci 51, 686-693.

Chevesich, J., Kreuz, A.J., and Montell, C. (1997). Requirement for the PDZ domain protein, INAD, for localization of the TRP store-operated channel to a signaling complex. Neuron 18, 95-105.

Chow, R.L., Snow, B., Novak, J., Looser, J., Freund, C., Vidgen, D., Ploder, L., and McInnes, R.R. (2001). Vsx1, a rapidly evolving paired-like homeobox gene expressed in cone bipolar cells. Mech Dev 109, 315-322.

Chu, X., Tong, Q., Wozney, J., Zhang, W., Cheung, J.Y., Conrad, K., Mazack, V., Stahl, R., Barber, D.L., and Miller, B.A. (2005). Identification of an N-terminal TRPC2 splice variant which inhibits calcium influx. Cell Calcium 37, 173-182.

Chuang, S.C., Bianchi, R., Kim, D., Shin, H.S., and Wong, R.K. (2001). Group I metabotropic glutamate receptors elicit epileptiform discharges in the hippocampus through PLCbetal signaling. J Neurosci 21, 6387-6394.

Claros, M.G., and von Heijne, G. (1994). TopPred II: an improved software for membrane protein structure predictions. Comput Appl Biosci 10, 685-686.

Cohen, E., and Sterling, P. (1990a). Convergence and divergence of cones onto bipolar cells in the central area of cat retina. Philos Trans R Soc Lond B Biol Sci 330, 323-328.

Cohen, E., and Sterling, P. (1990b). Demonstration of cell types among cone bipolar neurons of cat retina. Philos Trans R Soc Lond B Biol Sci 330, 305-321.

Connolly, T., and Gilmore, R. (1989). The signal recognition particle receptor mediates the GTP-dependent displacement of SRP from the signal sequence of the nascent polypeptide. Cell 57, 599-610.

Connolly, T., Rapiejko, P., and Gilmore, R. (1991). Requirement of GTP hydrolysis for dissociation of the signal recognition particle from its receptor. Science 252, 1171-1173.

Conzelmann, A., Puoti, A., Lester, R.L., and Desponds, C. (1992). Two different types of lipid moieties are present in glycophosphoinositol-anchored membrane proteins of Saccharomyces cerevisiae. EMBO J 11, 457-466.

Cserzo, M., Eisenhaber, F., Eisenhaber, B., and Simon, I. (2002). On filtering false positive transmembrane protein predictions. Protein Eng 15, 745-752.

Dacheux, R.F., and Raviola, E. (1986). The rod pathway in the rabbit retina: a depolarizing bipolar and amacrine cell. J Neurosci 6, 331-345. 
Dash, P.K., Karl, K.A., Colicos, M.A., Prywes, R., and Kandel, E.R. (1991). cAMP response element-binding protein is activated by $\mathrm{Ca} 2+/$ calmodulin- as well as cAMPdependent protein kinase. Proc Natl Acad Sci U S A 88, 5061-5065.

de la Villa, P., Kurahashi, T., and Kaneko, A. (1995). L-glutamate-induced responses and cGMP-activated channels in three subtypes of retinal bipolar cells dissociated from the cat. J Neurosci 15, 3571-3582.

Demas, J., Eglen, S.J., and Wong, R.O. (2003). Developmental loss of synchronous spontaneous activity in the mouse retina is independent of visual experience. $\mathrm{J}$ Neurosci $23,2851-2860$.

Demas, J., Sagdullaev, B.T., Green, E., Jaubert-Miazza, L., McCall, M.A., Gregg, R.G., Wong, R.O., and Guido, W. (2006). Failure to maintain eye-specific segregation in nob, a mutant with abnormally patterned retinal activity. Neuron 50, 247-259.

Denzer, A.J., Nabholz, C.E., and Spiess, M. (1995). Transmembrane orientation of signal-anchor proteins is affected by the folding state but not the size of the N-terminal domain. EMBO J 14, 6311-6317.

DeVries, S.H. (2000). Bipolar cells use kainate and AMPA receptors to filter visual information into separate channels. Neuron 28,847-856.

DeVries, S.H., Li, W., and Saszik, S. (2006). Parallel processing in two transmitter microenvironments at the cone photoreceptor synapse. Neuron 50, 735-748.

DeVries, S.H., and Schwartz, E.A. (1999). Kainate receptors mediate synaptic transmission between cones and 'Off' bipolar cells in a mammalian retina. Nature 397 , 157-160.

Dhingra, A., Jiang, M., Wang, T.L., Lyubarsky, A., Savchenko, A., Bar-Yehuda, T., Sterling, P., Birnbaumer, L., and Vardi, N. (2002). Light response of retinal ON bipolar cells requires a specific splice variant of Galpha(o). J Neurosci 22, 4878-4884.

Dhingra, A., Lyubarsky, A., Jiang, M., Pugh, E.N., Jr., Birnbaumer, L., Sterling, P., and Vardi, N. (2000). The light response of ON bipolar neurons requires G[alpha]o. J Neurosci 20, 9053-9058.

Doering, C.J., Rehak, R., Bonfield, S., Peloquin, J.B., Stell, W.K., Mema, S.C., Sauve, Y., and McRory, J.E. (2008). Modified Ca(v)1.4 expression in the Cacnalf(nob2) mouse due to alternative splicing of an ETn inserted in exon 2. PLoS One 3, e2538.

Dolmetsch, R.E., Lewis, R.S., Goodnow, C.C., and Healy, J.I. (1997). Differential activation of transcription factors induced by $\mathrm{Ca} 2+$ response amplitude and duration. Nature $386,855-858$. 
Dolmetsch, R.E., Xu, K., and Lewis, R.S. (1998). Calcium oscillations increase the efficiency and specificity of gene expression. Nature 392, 933-936.

Dong, X.P., Cheng, X., Mills, E., Delling, M., Wang, F., Kurz, T., and Xu, H. (2008). The type IV mucolipidosis-associated protein TRPML1 is an endolysosomal iron release channel. Nature 455, 992-996.

Dong, X.P., Wang, X., and Xu, H. (2010). TRP channels of intracellular membranes. J Neurochem 113, 313-328.

Dowling, J.E., and Ripps, H. (1970). Visual adaptation in the retina of the skate. J Gen Physiol 56, 491-520.

Dryja, T.P., Berson, E.L., Rao, V.R., and Oprian, D.D. (1993). Heterozygous missense mutation in the rhodopsin gene as a cause of congenital stationary night blindness. Nat Genet 4, 280-283.

Dryja, T.P., Hahn, L.B., Reboul, T., and Arnaud, B. (1996). Missense mutation in the gene encoding the alpha subunit of rod transducin in the Nougaret form of congenital stationary night blindness. Nat Genet 13, 358-360.

Duclos, F., and Koenig, M. (1995). Comparison of primary structure of a neuron-specific protein, X11, between human and mouse. Mamm Genome 6, 57-58.

Duncan, L.M., Deeds, J., Hunter, J., Shao, J., Holmgren, L.M., Woolf, E.A., Tepper, R.I., and Shyjan, A.W. (1998). Down-regulation of the novel gene melastatin correlates with potential for melanoma metastasis. Cancer Res 58, 1515-1520.

Duvoisin, R.M., Zhang, C., and Ramonell, K. (1995). A novel metabotropic glutamate receptor expressed in the retina and olfactory bulb. J Neurosci $15,3075-3083$.

Egea, P.F., Stroud, R.M., and Walter, P. (2005). Targeting proteins to membranes: structure of the signal recognition particle. Curr Opin Struct Biol 15, 213-220.

Euler, T., and Wassle, H. (1995). Immunocytochemical identification of cone bipolar cells in the rat retina. J Comp Neurol 361, 461-478.

Famiglietti, E.V., Jr., and Kolb, H. (1975). A bistratified amacrine cell and synaptic cirucitry in the inner plexiform layer of the retina. Brain Res 84, 293-300.

Famiglietti, E.V., Jr., and Kolb, H. (1976). Structural basis for ON-and OFF-center responses in retinal ganglion cells. Science 194, 193-195.

Fasolato, C., Hoth, M., and Penner, R. (1993). A GTP-dependent step in the activation mechanism of capacitative calcium influx. J Biol Chem 268, 20737-20740. 
Feller, M.B. (1999). Spontaneous correlated activity in developing neural circuits. Neuron 22, 653-656.

Feller, M.B., Wellis, D.P., Stellwagen, D., Werblin, F.S., and Shatz, C.J. (1996). Requirement for cholinergic synaptic transmission in the propagation of spontaneous retinal waves. Science 272, 1182-1187.

Ferguson, M.A., Brimacombe, J.S., Brown, J.R., Crossman, A., Dix, A., Field, R.A., Guther, M.L., Milne, K.G., Sharma, D.K., and Smith, T.K. (1999). The GPI biosynthetic pathway as a therapeutic target for African sleeping sickness. Biochim Biophys Acta $1455,327-340$.

Fernando, K.C., Gregory, R.B., Katsis, F., Kemp, B.E., and Barritt, G.J. (1997). Evidence that a low-molecular-mass GTP-binding protein is required for store-activated $\mathrm{Ca} 2+$ inflow in hepatocytes. Biochem J 328 ( Pt 2), 463-471.

Fields, R.D., Eshete, F., Stevens, B., and Itoh, K. (1997). Action potential-dependent regulation of gene expression: temporal specificity in ca2+, cAMP-responsive element binding proteins, and mitogen-activated protein kinase signaling. J Neurosci 17, $7252-$ 7266.

Fisher, L.J. (1979a). Development of synaptic arrays in the inner plexiform layer of neonatal mouse retina. J Comp Neurol 187, 359-372.

Fisher, L.J. (1979b). Interplexiform cell of the mouse retina: a Golgi demonstration. Invest Ophthalmol Vis Sci 18, 521-523.

Fisher, M.A. (1979c). F waves: comments on the central control of recurrent discharges. Muscle Nerve 2, 406.

Fletcher, E.L., Koulen, P., and Wassle, H. (1998). GABAA and GABAC receptors on mammalian rod bipolar cells. J Comp Neurol 396, 351-365.

Freymann, D.M., Keenan, R.J., Stroud, R.M., and Walter, P. (1999). Functional changes in the structure of the SRP GTPase on binding GDP and $\mathrm{Mg} 2+$ GDP. Nat Struct Biol 6, 793-801.

Galli, L., and Maffei, L. (1988). Spontaneous impulse activity of rat retinal ganglion cells in prenatal life. Science 242, 90-91.

Garnier, C., Blondel, M.O., and Haguenauer-Tsapis, R. (1996). Membrane topology of the yeast uracil permease. Mol Microbiol 21, 1061-1073.

Ghosh, K.K., Bujan, S., Haverkamp, S., Feigenspan, A., and Wassle, H. (2004). Types of bipolar cells in the mouse retina. J Comp Neurol 469, 70-82. 
Gilbertson, T.A., Scobey, R., and Wilson, M. (1991). Permeation of calcium ions through non-NMDA glutamate channels in retinal bipolar cells. Science 251, 1613-1615.

Gilmore, R., Walter, P., and Blobel, G. (1982). Protein translocation across the endoplasmic reticulum. II. Isolation and characterization of the signal recognition particle receptor. J Cell Biol 95, 470-477.

Goldberg, J.L., Espinosa, J.S., Xu, Y., Davidson, N., Kovacs, G.T., and Barres, B.A. (2002). Retinal ganglion cells do not extend axons by default: promotion by neurotrophic signaling and electrical activity. Neuron 33, 689-702.

Goldoni, S., Owens, R.T., McQuillan, D.J., Shriver, Z., Sasisekharan, R., Birk, D.E., Campbell, S., and Iozzo, R.V. (2004). Biologically active decorin is a monomer in solution. J Biol Chem 279, 6606-6612.

Gonzalez-Islas, C., and Wenner, P. (2006). Spontaneous network activity in the embryonic spinal cord regulates AMPAergic and GABAergic synaptic strength. Neuron $49,563-575$.

Granit, R. (1947). Visual cells of the guinea pig. Nature 160, 838.

Greferath, U., Grunert, U., and Wassle, H. (1990). Rod bipolar cells in the mammalian retina show protein kinase C-like immunoreactivity. J Comp Neurol 301, 433-442.

Gregg, R.G., Kamermans, M., Klooster, J., Lukasiewicz, P.D., Peachey, N.S., Vessey, K.A., and McCall, M.A. (2007). Nyctalopin expression in retinal bipolar cells restores visual function in a mouse model of complete X-linked congenital stationary night blindness. J Neurophysiol 98, 3023-3033.

Gregg, R.G., Mukhopadhyay, S., Candille, S.I., Ball, S.L., Pardue, M.T., McCall, M.A., and Peachey, N.S. (2003a). Identification of the gene and the mutation responsible for the mouse nob phenotype. Invest Ophthalmol Vis Sci 44, 378-384.

Gregg, R.G., Willer, G.B., Fadool, J.M., Dowling, J.E., and Link, B.A. (2003b). Positional cloning of the young mutation identifies an essential role for the Brahma chromatin remodeling complex in mediating retinal cell differentiation. Proc Natl Acad Sci U S A 100, 6535-6540.

Guidetti, G.F., Bartolini, B., Bernardi, B., Tira, M.E., Berndt, M.C., Balduini, C., and Torti, M. (2004). Binding of von Willebrand factor to the small proteoglycan decorin. FEBS Lett 574, 95-100.

Guillaud, L., Wong, R., and Hirokawa, N. (2008). Disruption of KIF17-Mint1 interaction by CaMKII-dependent phosphorylation: a molecular model of kinesin-cargo release. Nat Cell Biol 10, 19-29. 
Hack, I., Peichl, L., and Brandstatter, J.H. (1999). An alternative pathway for rod signals in the rodent retina: rod photoreceptors, cone bipolar cells, and the localization of glutamate receptors. Proc Natl Acad Sci U S A 96, 14130-14135.

Haeseleer, F., Imanishi, Y., Maeda, T., Possin, D.E., Maeda, A., Lee, A., Rieke, F., and Palczewski, K. (2004). Essential role of Ca2+-binding protein 4, a Cav1.4 channel regulator, in photoreceptor synaptic function. Nat Neurosci 7, 1079-1087.

Haeseleer, F., and Palczewski, K. (2000). Short-chain dehydrogenases/reductases in retina. Methods Enzymol 316, 372-383.

Hanson, M.G., and Landmesser, L.T. (2004). Normal patterns of spontaneous activity are required for correct motor axon guidance and the expression of specific guidance molecules. Neuron 43, 687-701.

Hanson, M.G., Milner, L.D., and Landmesser, L.T. (2008). Spontaneous rhythmic activity in early chick spinal cord influences distinct motor axon pathfinding decisions. Brain Res Rev 57, 77-85.

Hardie, R.C. (1995). Photolysis of caged Ca2+ facilitates and inactivates but does not directly excite light-sensitive channels in Drosophila photoreceptors. J Neurosci 15, 889902.

Hardie, R.C. (1996a). Calcium signalling: setting store by calcium channels. Curr Biol 6, 1371-1373.

Hardie, R.C. (1996b). Excitation of Drosophila photoreceptors by BAPTA and ionomycin: evidence for capacitative Ca2+ entry? Cell Calcium 20,315-327.

Hardie, R.C. (2003). Regulation of TRP channels via lipid second messengers. Annu Rev Physiol 65, 735-759.

Hardie, R.C., and Raghu, P. (1998). Activation of heterologously expressed Drosophila TRPL channels: $\mathrm{Ca} 2+$ is not required and InsP3 is not sufficient. Cell Calcium 24, 153 163.

Harris, W.A., and Holt, C.E. (1990). Early events in the embryogenesis of the vertebrate visual system: cellular determination and pathfinding. Annu Rev Neurosci 13, 155-169.

Harris, W.S., and Kogan, I. (1981). Retrospective study of retinal complications with posterior chamber lenses. Contact Intraocul Lens Med J 7, 341-344.

Hartmann, E., Rapoport, T.A., and Lodish, H.F. (1989). Predicting the orientation of eukaryotic membrane-spanning proteins. Proc Natl Acad Sci U S A 86, 5786-5790. 
Hartveit, E. (1997). Functional organization of cone bipolar cells in the rat retina. J Neurophysiol 77, 1716-1730.

Haverkamp, S., Haeseleer, F., and Hendrickson, A. (2003). A comparison of immunocytochemical markers to identify bipolar cell types in human and monkey retina. Vis Neurosci 20, 589-600.

He, W., Cowan, C.W., and Wensel, T.G. (1998). RGS9, a GTPase accelerator for phototransduction. Neuron 20,95-102.

Heesen, S., Lehle, L., Weissmann, A., and Aebi, M. (1994). Isolation of the ALG5 locus encoding the UDP-glucose:dolichyl-phosphate glucosyltransferase from Saccharomyces cerevisiae. Eur J Biochem 224, 71-79.

Heidelberger, R., and Matthews, G. (1992). Calcium influx and calcium current in single synaptic terminals of goldfish retinal bipolar neurons. J Physiol 447, 235-256.

Heijne, G. (1986). The distribution of positively charged residues in bacterial inner membrane proteins correlates with the trans-membrane topology. EMBO J 5, 3021-3027.

Hendrickson, A.E. (1996). Synaptic development in macaque monkey retina and its implications for other developmental sequences. Perspect Dev Neurobiol 3, 195-201.

Henry, D., Burke, S., Shishido, E., and Matthews, G. (2003). Retinal bipolar neurons express the cyclic nucleotide-gated channel of cone photoreceptors. J Neurophysiol 89 , 754-761.

Hirokawa, T., Boon-Chieng, S., and Mitaku, S. (1998). SOSUI: classification and secondary structure prediction system for membrane proteins. Bioinformatics 14,378 379.

Hood, D.C., and Birch, D.G. (1996). Assessing abnormal rod photoreceptor activity with the a-wave of the electroretinogram: applications and methods. Doc Ophthalmol 92, 253267.

Huang, L., Max, M., Margolskee, R.F., Su, H., Masland, R.H., and Euler, T. (2003). G protein subunit $G$ gamma 13 is coexpressed with $G$ alpha o, $G$ beta 3 , and $G$ beta 4 in retinal ON bipolar cells. J Comp Neurol 455, 1-10.

Ikezawa, H. (2002). Glycosylphosphatidylinositol (GPI)-anchored proteins. Biol Pharm Bull 25, 409-417.

Inouye, H., and Beckwith, J. (1977). Synthesis and processing of an Escherichia coli alkaline phosphatase precursor in vitro. Proc Natl Acad Sci U S A 74, 1440-1444. 
Inouye, S., Wang, S., Sekizawa, J., Halegoua, S., and Inouye, M. (1977). Amino acid sequence for the peptide extension on the prolipoprotein of the Escherichia coli outer membrane. Proc Natl Acad Sci U S A 74, 1004-1008.

Irvine, R.F. (1990). 'Quantal' Ca2+ release and the control of $\mathrm{Ca} 2+$ entry by inositol phosphates--a possible mechanism. FEBS Lett 263, 5-9.

Ishii, M., Morigiwa, K., Takao, M., Nakanishi, S., Fukuda, Y., Mimura, O., and Tsukamoto, Y. (2009). Ectopic synaptic ribbons in dendrites of mouse retinal ON- and OFF-bipolar cells. Cell Tissue Res 338, 355-375.

Jaffe, A.B., and Hall, A. (2005). Rho GTPases: biochemistry and biology. Annu Rev Cell Dev Biol 21, 247-269.

Jahn, R., and Scheller, R.H. (2006). SNAREs--engines for membrane fusion. Nat Rev Mol Cell Biol 7, 631-643.

Johnsson, N., and Varshavsky, A. (1994). Split ubiquitin as a sensor of protein interactions in vivo. Proc Natl Acad Sci U S A 91, 10340-10344.

Kajava, A.V. (1998). Structural diversity of leucine-rich repeat proteins. J Mol Biol 277, 519-527.

Kaneko, A., and Saito, T. (1983). Ionic mechanisms underlying the responses of offcenter bipolar cells in the carp retina. II. Studies on responses evoked by transretinal current stimulation. J Gen Physiol 81, 603-612.

Kanzaki, M., Zhang, Y.Q., Mashima, H., Li, L., Shibata, H., and Kojima, I. (1999). Translocation of a calcium-permeable cation channel induced by insulin-like growth factor-I. Nat Cell Biol I, 165-170.

Kim, D.S., Ross, S.E., Trimarchi, J.M., Aach, J., Greenberg, M.E., and Cepko, C.L. (2008). Identification of molecular markers of bipolar cells in the murine retina. J Comp Neurol 507, 1795-1810.

Kobe, B., and Deisenhofer, J. (1994). The leucine-rich repeat: a versatile binding motif. Trends Biochem Sci 19, 415-421.

Kobe, B., and Deisenhofer, J. (1996). Mechanism of ribonuclease inhibition by ribonuclease inhibitor protein based on the crystal structure of its complex with ribonuclease A. J Mol Biol 264, 1028-1043.

Kohl, S., Baumann, B., Broghammer, M., Jagle, H., Sieving, P., Kellner, U., Spegal, R., Anastasi, M., Zrenner, E., Sharpe, L.T., et al. (2000). Mutations in the CNGB3 gene encoding the beta-subunit of the cone photoreceptor cGMP-gated channel are responsible for achromatopsia (ACHM3) linked to chromosome 8q21. Hum Mol Genet 9, 2107-2116. 
Kohl, S., Marx, T., Giddings, I., Jagle, H., Jacobson, S.G., Apfelstedt-Sylla, E., Zrenner, E., Sharpe, L.T., and Wissinger, B. (1998). Total colourblindness is caused by mutations in the gene encoding the alpha-subunit of the cone photoreceptor cGMP-gated cation channel. Nat Genet 19, 257-259.

Koike, C., Obara, T., Uriu, Y., Numata, T., Sanuki, R., Miyata, K., Koyasu, T., Ueno, S., Funabiki, K., Tani, A., et al. (2010). TRPMl is a component of the retinal ON bipolar cell transduction channel in the mGluR6 cascade. Proc Natl Acad Sci U S A 107, 332337.

Kolb, H. (1977). The organization of the outer plexiform layer in the retina of the cat: electron microscopic observations. J Neurocytol 6, 131-153.

Kolb, H., and Famiglietti, E.V. (1974). Rod and cone pathways in the inner plexiform layer of cat retina. Science 186, 47-49.

Kolb, H., and Jones, J. (1984). Synaptic organization of the outer plexiform layer of the turtle retina: an electron microscope study of serial sections. J Neurocytol 13, 567-591.

Kolb, H., and West, R.W. (1977). Synaptic connections of the interplexiform cell in the retina of the cat. J Neurocytol 6, 155-170.

Koulen, P., Garner, C.C., and Wassle, H. (1998). Immunocytochemical localization of the synapse-associated protein SAP102 in the rat retina. J Comp Neurol 397, 326-336.

Krapivinsky, G., Mochida, S., Krapivinsky, L., Cibulsky, S.M., and Clapham, D.E. (2006). The TRPM7 ion channel functions in cholinergic synaptic vesicles and affects transmitter release. Neuron 52, 485-496.

Kriegstein, A.R., Owens, D.F., and Avoli, M. (1999). Ontogeny of channels, transmitters and epileptogenesis. Adv Neurol 79, 145-159.

Krogh, A., Larsson, B., von Heijne, G., and Sonnhammer, E.L. (2001). Predicting transmembrane protein topology with a hidden Markov model: application to complete genomes. J Mol Biol 305, 567-580.

Le Goff, M.M., and Bishop, P.N. (2007). Focus on molecules: opticin. Exp Eye Res 85, 303-304.

Le Goff, M.M., Hindson, V.J., Jowitt, T.A., Scott, P.G., and Bishop, P.N. (2003). Characterization of opticin and evidence of stable dimerization in solution. J Biol Chem $278,45280-45287$.

Lee, J.C., Gimm, J.A., Lo, A.J., Koury, M.J., Krauss, S.W., Mohandas, N., and Chasis, J.A. (2004). Mechanism of protein sorting during erythroblast enucleation: role of cytoskeletal connectivity. Blood 103, 1912-1919. 
Leinekugel, X. (2003). Developmental patterns and plasticities: the hippocampal model. J Physiol Paris 97, 27-37.

Li, Y.W., and Bayliss, D.A. (1998). Electrophysical properties, synaptic transmission and neuromodulation in serotonergic caudal raphe neurons. Clin Exp Pharmacol Physiol 25, 468-473.

Liebman, P.A., and Pugh, E.N., Jr. (1980). ATP mediates rapid reversal of cyclic GMP phosphodiesterase activation in visual receptor membranes. Nature $287,734-736$.

Lillien, L. (1994). Neurogenesis in the vertebrate retina. Perspect Dev Neurobiol 2, 175 182.

Lipp, J., and Dobberstein, B. (1986). Signal recognition particle-dependent membrane insertion of mouse invariant chain: a membrane-spanning protein with a cytoplasmically exposed amino terminus. J Cell Biol 102, 2169-2175.

Lockwich, T., Singh, B.B., Liu, X., and Ambudkar, I.S. (2001). Stabilization of cortical actin induces internalization of transient receptor potential $3(\operatorname{Trp} 3)$-associated caveolar $\mathrm{Ca} 2+$ signaling complex and loss of $\mathrm{Ca} 2+$ influx without disruption of Trp3-inositol trisphosphate receptor association. J Biol Chem 276, 42401-42408.

Lyko, F., Martoglio, B., Jungnickel, B., Rapoport, T.A., and Dobberstein, B. (1995). Signal sequence processing in rough microsomes. J Biol Chem 270, 19873-19878.

MacNeil, M.A., Heussy, J.K., Dacheux, R.F., Raviola, E., and Masland, R.H. (2004). The population of bipolar cells in the rabbit retina. J Comp Neurol 472, 73-86.

Maddox, D.M., Vessey, K.A., Yarbrough, G.L., Invergo, B.M., Cantrell, D.R., Inayat, S., Balannik, V., Hicks, W.L., Hawes, N.L., Byers, S., et al. (2008). Allelic variance between GRM6 mutants, Grm6nob3 and Grm6nob4 results in differences in retinal ganglion cell visual responses. J Physiol 586, 4409-4424.

Manookin, M.B., Beaudoin, D.L., Ernst, Z.R., Flagel, L.J., and Demb, J.B. (2008). Disinhibition combines with excitation to extend the operating range of the OFF visual pathway in daylight. J Neurosci $28,4136-4150$.

Mariani, A.P. (1981). A diffuse, invaginating cone bipolar cell in primate retina. J Comp Neurol 197, 661-671.

Mariani, A.P. (1985). Multiaxonal horizontal cells in the retina of the tree shrew, Tupaia glis. J Comp Neurol 233, 553-563.

Marquardt, T., and Gruss, P. (2002). Generating neuronal diversity in the retina: one for nearly all. Trends Neurosci $25,32-38$. 
Marshak, D.W., and Dowling, J.E. (1987). Synapses of cone horizontal cell axons in goldfish retina. J Comp Neurol 256, 430-443.

Martoglio, B., and Dobberstein, B. (1998). Signal sequences: more than just greasy peptides. Trends Cell Biol 8, 410-415.

Masland, R.H. (1977). Maturation of function in the developing rabbit retina. J Comp Neurol 175, 275-286.

Masu, M., Iwakabe, H., Tagawa, Y., Miyoshi, T., Yamashita, M., Fukuda, Y., Sasaki, H., Hiroi, K., Nakamura, Y., Shigemoto, R., et al. (1995). Specific deficit of the ON response in visual transmission by targeted disruption of the mGluR6 gene. Cell 80, 757-765.

Masuho, I., Celver, J., Kovoor, A., and Martemyanov, K.A. (2010). Membrane anchor R9AP potentiates GTPase-accelerating protein activity of RGS11 x Gbeta5 complex and accelerates inactivation of the mGluR6-G(o) signaling. J Biol Chem 285, 4781-4787.

McConville, M.J., Collidge, T.A., Ferguson, M.A., and Schneider, P. (1993). The glycoinositol phospholipids of Leishmania mexicana promastigotes. Evidence for the presence of three distinct pathways of glycolipid biosynthesis. J Biol Chem 268, 15595 15604.

McEwan, P.A., Scott, P.G., Bishop, P.N., and Bella, J. (2006). Structural correlations in the family of small leucine-rich repeat proteins and proteoglycans. J Struct Biol 155, 294305.

McGuire, B.A., Hornung, J.P., Gilbert, C.D., and Wiesel, T.N. (1984). Patterns of synaptic input to layer 4 of cat striate cortex. J Neurosci 4, 3021-3033.

Meadows, L.A., Gell, D., Broadie, K., Gould, A.P., and White, R.A. (1994). The cell adhesion molecule, connectin, and the development of the Drosophila neuromuscular system. J Cell Sci 107 ( Pt 1), 321-328.

Mehta, D., Ahmmed, G.U., Paria, B.C., Holinstat, M., Voyno-Yasenetskaya, T., Tiruppathi, C., Minshall, R.D., and Malik, A.B. (2003). RhoA interaction with inositol 1,4,5-trisphosphate receptor and transient receptor potential channel-1 regulates $\mathrm{Ca} 2+$ entry. Role in signaling increased endothelial permeability. J Biol Chem 278, 3349233500 .

Meister, M., Wong, R.O., Baylor, D.A., and Shatz, C.J. (1991). Synchronous bursts of action potentials in ganglion cells of the developing mammalian retina. Science 252, 939943.

Mellman, I., and Nelson, W.J. (2008). Coordinated protein sorting, targeting and distribution in polarized cells. Nat Rev Mol Cell Biol 9, 833-845. 
Mende, U., Zagrovic, B., Cohen, A., Li, Y., Valenzuela, D., Fishman, M.C., and Neer, E.J. (1998). Effect of deletion of the major brain G-protein alpha subunit (alpha(o)) on coordination of G-protein subunits and on adenylyl cyclase activity. J Neurosci Res 54, 263-272.

Mery, L., Strauss, B., Dufour, J.F., Krause, K.H., and Hoth, M. (2002). The PDZinteracting domain of TRPC4 controls its localization and surface expression in HEK293 cells. J Cell Sci 115, 3497-3508.

Mey, J., and Thanos, S. (1992). Development of the visual system of the chick--a review. J Hirnforsch 33, 673-702.

Miller, K.D., Erwin, E., and Kayser, A. (1999). Is the development of orientation selectivity instructed by activity? J Neurobiol $41,44-57$.

Miller, R.F., and Dowling, J.E. (1970). Intracellular responses of the Muller (glial) cells of mudpuppy retina: their relation to b-wave of the electroretinogram. J Neurophysiol 33, 323-341.

Miyake, Y., Horiguchi, M., Ota, I., and Shiroyama, N. (1987a). Characteristic ERGflicker anomaly in incomplete congenital stationary night blindness. Invest Ophthalmol Vis Sci $28,1816-1823$.

Miyake, Y., Yagasaki, K., and Horiguchi, M. (1987b). A rod-cone dysfunction syndrome with separate clinical entity: incomplete-type congenital stationary night blindness (Miyake). Prog Clin Biol Res 247, 137-145.

Miyake, Y., Yagasaki, K., Horiguchi, M., and Kawase, Y. (1987c). On- and off-responses in photopic electroretinogram in complete and incomplete types of congenital stationary night blindness. Jpn J Ophthalmol 31, 81-87.

Mohajerani, M.H., and Cherubini, E. (2006). Role of giant depolarizing potentials in shaping synaptic currents in the developing hippocampus. Crit Rev Neurobiol 18, 13-23.

Montell, C., and Rubin, G.M. (1989). Molecular characterization of the Drosophila trp locus: a putative integral membrane protein required for phototransduction. Neuron 2 , 1313-1323.

Morenilla-Palao, C., Planells-Cases, R., Garcia-Sanz, N., and Ferrer-Montiel, A. (2004). Regulated exocytosis contributes to protein kinase $\mathrm{C}$ potentiation of vanilloid receptor activity. J Biol Chem 279, 25665-25672.

Morgan, J., Huckfeldt, R., and Wong, R.O. (2005). Imaging techniques in retinal research. Exp Eye Res 80, 297-306. 
Morgan, J.L., Dhingra, A., Vardi, N., and Wong, R.O. (2006). Axons and dendrites originate from neuroepithelial-like processes of retinal bipolar cells. Nat Neurosci 9, 8592.

Morgans, C.W., Bayley, P.R., Oesch, N.W., Ren, G., Akileswaran, L., and Taylor, W.R. (2005). Photoreceptor calcium channels: insight from night blindness. Vis Neurosci 22, 561-568.

Morgans, C.W., Brandstatter, J.H., Kellerman, J., Betz, H., and Wassle, H. (1996). A SNARE complex containing syntaxin 3 is present in ribbon synapses of the retina. $J$ Neurosci 16, 6713-6721.

Morgans, C.W., El Far, O., Berntson, A., Wassle, H., and Taylor, W.R. (1998). Calcium extrusion from mammalian photoreceptor terminals. J Neurosci 18, 2467-2474.

Morgans, C.W., Ren, G., and Akileswaran, L. (2006). Localization of nyctalopin in the mammalian retina. Eur J Neurosci 23, 1163-1171.

Morgans, C.W., Wensel, T.G., Brown, R.L., Perez-Leon, J.A., Bearnot, B., and Duvoisin, R.M. (2007). Gbeta5-RGS complexes co-localize with mGluR6 in retinal ON-bipolar cells. Eur J Neurosci 26, 2899-2905.

Morgans, C.W., Zhang, J., Jeffrey, B.G., Nelson, S.M., Burke, N.S., Duvoisin, R.M., and Brown, R.L. (2009). TRPM1 is required for the depolarizing light response in retinal ONbipolar cells. Proc Natl Acad Sci U S A 106, 19174-19178.

Mumm, J.S., Godinho, L., Morgan, J.L., Oakley, D.M., Schroeter, E.H., and Wong, R.O. (2005). Laminar circuit formation in the vertebrate retina. Prog Brain Res 147, 155-169.

Myers, B.R., Bohlen, C.J., and Julius, D. (2008). A yeast genetic screen reveals a critical role for the pore helix domain in TRP channel gating. Neuron 58, 362-373.

Nachman-Clewner, M., St Jules, R., and Townes-Anderson, E. (1999). L-type calcium channels in the photoreceptor ribbon synapse: localization and role in plasticity. J Comp Neurol 415, 1-16.

Nakajima, Y., Iwakabe, H., Akazawa, C., Nawa, H., Shigemoto, R., Mizuno, N., and Nakanishi, S. (1993). Molecular characterization of a novel retinal metabotropic glutamate receptor mGluR6 with a high agonist selectivity for L-2-amino-4phosphonobutyrate. J Biol Chem 268, 11868-11873.

Nakamura, M., Hotta, Y., Tanikawa, A., Terasaki, H., and Miyake, Y. (2000). A high association with cone dystrophy in Fundus albipunctatus caused by mutations of the RDH5 gene. Invest Ophthalmol Vis Sci 41, 3925-3932. 
Nakanishi, S. (1994). Metabotropic glutamate receptors: synaptic transmission, modulation, and plasticity. Neuron 13, 1031-1037.

Nawy, S. (1999). The metabotropic receptor mGluR6 may signal through G(o), but not phosphodiesterase, in retinal bipolar cells. J Neurosci 19, 2938-2944.

Nawy, S. (2000). Regulation of the on bipolar cell mGluR6 pathway by Ca2+. J Neurosci 20, 4471-4479.

Nawy, S., and Jahr, C.E. (1991). cGMP-gated conductance in retinal bipolar cells is suppressed by the photoreceptor transmitter. Neuron 7, 677-683.

Nelson, R. (1977). Cat cones have rod input: a comparison of the response properties of cones and horizontal cell bodies in the retina of the cat. J Comp Neurol 172, 109-135.

Nicol, X., Voyatzis, S., Muzerelle, A., Narboux-Neme, N., Sudhof, T.C., Miles, R., and Gaspar, P. (2007). cAMP oscillations and retinal activity are permissive for ephrin signaling during the establishment of the retinotopic map. Nat Neurosci 10, 340-347.

Nilius, B., Prenen, J., Janssens, A., Owsianik, G., Wang, C., Zhu, M.X., and Voets, T. (2005). The selectivity filter of the cation channel TRPM4. J Biol Chem 280, 2289922906.

Nishimura, Y., and Rakic, P. (1987). Synaptogenesis in the primate retina proceeds from the ganglion cells towards the photoreceptors. Neurosci Res Suppl 6, S253-268.

Noell, W.K. (1953). Experimentally induced toxic effects on structure and function of visual cells and pigment epithelium. Am J Ophthalmol 36, 103-116.

Nomura, A., Shigemoto, R., Nakamura, Y., Okamoto, N., Mizuno, N., and Nakanishi, S. (1994). Developmentally regulated postsynaptic localization of a metabotropic glutamate receptor in rat rod bipolar cells. Cell 77, 361-369.

O'Connor, E., Eisenhaber, B., Dalley, J., Wang, T., Missen, C., Bulleid, N., Bishop, P.N., and Trump, D. (2005). Species specific membrane anchoring of nyctalopin, a small leucine-rich repeat protein. Hum Mol Genet 14, 1877-1887.

Oancea, E., Vriens, J., Brauchi, S., Jun, J., Splawski, I., and Clapham, D.E. (2009). TRPM1 forms ion channels associated with melanin content in melanocytes. Sci Signal 2, ra21.

Obukhov, A.G., and Nowycky, M.C. (2004). TRPC5 activation kinetics are modulated by the scaffolding protein ezrin/radixin/moesin-binding phosphoprotein-50 (EBP50). J Cell Physiol 201, 227-235. 
Ohtoshi, A., Justice, M.J., and Behringer, R.R. (2001). Isolation and characterization of Vsx1, a novel mouse CVC paired-like homeobox gene expressed during embryogenesis and in the retina. Biochem Biophys Res Commun 286, 133-140.

Okamoto, M., and Sudhof, T.C. (1997). Mints, Munc18-interacting proteins in synaptic vesicle exocytosis. J Biol Chem 272, 31459-31464.

Okamoto, M., and Sudhof, T.C. (1998). Mint 3: a ubiquitous mint isoform that does not bind to munc18-1 or -2. Eur J Cell Biol 77, 161-165.

Paillart, C., Li, J., Matthews, G., and Sterling, P. (2003). Endocytosis and vesicle recycling at a ribbon synapse. J Neurosci $23,4092-4099$.

Palczewski, K., Buczylko, J., Van Hooser, P., Carr, S.A., Huddleston, M.J., and Crabb, J.W. (1992). Identification of the autophosphorylation sites in rhodopsin kinase. J Biol Chem 267, 18991-18998.

Panzner, S., Dreier, L., Hartmann, E., Kostka, S., and Rapoport, T.A. (1995). Posttranslational protein transport into the endoplasmic reticulum. Cold Spring Harb Symp Quant Biol 60, 31-40.

Pardue, M.T., McCall, M.A., LaVail, M.M., Gregg, R.G., and Peachey, N.S. (1998). A naturally occurring mouse model of $\mathrm{X}$-linked congenital stationary night blindness. Invest Ophthalmol Vis Sci 39, 2443-2449.

Parekh, A.B., and Putney, J.W., Jr. (2005). Store-operated calcium channels. Physiol Rev $85,757-810$.

Parsons, T.D., and Sterling, P. (2003). Synaptic ribbon. Conveyor belt or safety belt? Neuron 37, 379-382.

Patterson, R.L., van Rossum, D.B., and Gill, D.L. (1999). Store-operated Ca2+ entry: evidence for a secretion-like coupling model. Cell 98, 487-499.

Pearse, B.M., and Robinson, M.S. (1990). Clathrin, adaptors, and sorting. Annu Rev Cell Biol 6, 151-171.

Pennesi, M.E., Lyubarsky, A.L., and Pugh, E.N., Jr. (1998). Extreme responsiveness of the pupil of the dark-adapted mouse to steady retinal illumination. Invest Ophthalmol Vis Sci 39, 2148-2156.

Pignatelli, V., and Strettoi, E. (2004). Bipolar cells of the mouse retina: a gene gun, morphological study. J Comp Neurol 476, 254-266.

Pinto, L.H., Vitaterna, M.H., Shimomura, K., Siepka, S.M., Balannik, V., McDearmon, E.L., Omura, C., Lumayag, S., Invergo, B.M., Glawe, B., et al. (2007). Generation, 
identification and functional characterization of the nob4 mutation of Grm6 in the mouse. Vis Neurosci 24, 111-123.

Prada, C., Puga, J., Perez-Mendez, L., Lopez, R., and Ramirez, G. (1991). Spatial and Temporal Patterns of Neurogenesis in the Chick Retina. Eur J Neurosci 3, 559-569.

Pusch, C.M., Zeitz, C., Brandau, O., Pesch, K., Achatz, H., Feil, S., Scharfe, C., Maurer, J., Jacobi, F.K., Pinckers, A., et al. (2000). The complete form of X-linked congenital stationary night blindness is caused by mutations in a gene encoding a leucine-rich repeat protein. Nat Genet 26, 324-327.

Raghu, P., Colley, N.J., Webel, R., James, T., Hasan, G., Danin, M., Selinger, Z., and Hardie, R.C. (2000a). Normal phototransduction in Drosophila photoreceptors lacking an InsP(3) receptor gene. Mol Cell Neurosci 15, 429-445.

Raghu, P., Usher, K., Jonas, S., Chyb, S., Polyanovsky, A., and Hardie, R.C. (2000b). Constitutive activity of the light-sensitive channels TRP and TRPL in the Drosophila diacylglycerol kinase mutant, rdgA. Neuron 26, 169-179.

Rao, A., Dallman, R., Henderson, S., and Chen, C.K. (2007). Gbeta5 is required for normal light responses and morphology of retinal ON-bipolar cells. J Neurosci 27, 14199-14204.

Rapoport, T.A., Jungnickel, B., and Kutay, U. (1996a). Protein transport across the eukaryotic endoplasmic reticulum and bacterial inner membranes. Annu Rev Biochem $65,271-303$.

Rapoport, T.A., Rolls, M.M., and Jungnickel, B. (1996b). Approaching the mechanism of protein transport across the ER membrane. Curr Opin Cell Biol 8, 499-504.

Ridley, A.J. (2001). Rho proteins: linking signaling with membrane trafficking. Traffic 2 , 303-310.

Riggs, L.A. (1965). Vertebrate Color Receptors. Science 147, 913.

Riggs, L.A. (1954). Electroretinography in cases of night blindness. Am J Ophthalmol $38,70-78$.

Romano, C., Yang, W.L., and O'Malley, K.L. (1996). Metabotropic glutamate receptor 5 is a disulfide-linked dimer. J Biol Chem 271, 28612-28616.

Rosner, M., Hefetz, L., and Abraham, F.A. (1993). The prevalence of retinitis pigmentosa and congenital stationary night blindness in Israel. Am J Ophthalmol 116, 373-374.

Rothman, J.E. (1994). Mechanisms of intracellular protein transport. Nature 372, 55-63. 
Sabatini, D.D., Blobel, G., Nonomura, Y., and Adelman, M.R. (1971). Ribosomemembrane interaction: Structural aspects and functional implications. Adv Cytopharmacol 1, 119-129.

Saito, T., and Kaneko, A. (1983). Ionic mechanisms underlying the responses of offcenter bipolar cells in the carp retina. I. Studies on responses evoked by light. J Gen Physiol 81, 589-601.

Sakaguchi, M., Tomiyoshi, R., Kuroiwa, T., Mihara, K., and Omura, T. (1992). Functions of signal and signal-anchor sequences are determined by the balance between the hydrophobic segment and the N-terminal charge. Proc Natl Acad Sci U S A 89, 16-19.

Sanders, S.L., and Schekman, R. (1992). Polypeptide translocation across the endoplasmic reticulum membrane. J Biol Chem 267, 13791-13794.

Schaefer, L., and Iozzo, R.V. (2008). Biological functions of the small leucine-rich proteoglycans: from genetics to signal transduction. J Biol Chem 283, 21305-21309.

Schmitz, F., Konigstorfer, A., and Sudhof, T.C. (2000). RIBEYE, a component of synaptic ribbons: a protein's journey through evolution provides insight into synaptic ribbon function. Neuron $28,857-872$.

Schubert, G., and Bornschein, H. (1952). [Analysis of the human electroretinogram.]. Ophthalmologica 123, 396-413.

Schulte, D., and Bumsted-O'Brien, K.M. (2008). Molecular mechanisms of vertebrate retina development: implications for ganglion cell and photoreceptor patterning. Brain Res 1192, 151-164.

Schwartz, T.H., Rabinowitz, D., Unni, V., Kumar, V.S., Smetters, D.K., Tsiola, A., and Yuste, R. (1998). Networks of coactive neurons in developing layer 1. Neuron 20, 541552.

Scott, P.G., Bishop, P.N., and Bella, J. (2006a). On the calculation of the binding force between decorin and collagen. J Biomech 39, 1159-1160; author reply 1160-1152.

Scott, P.G., Dodd, C.M., Bergmann, E.M., Sheehan, J.K., and Bishop, P.N. (2006b). Crystal structure of the biglycan dimer and evidence that dimerization is essential for folding and stability of class I small leucine-rich repeat proteoglycans. J Biol Chem 281, 13324-13332.

Scott, P.G., Grossmann, J.G., Dodd, C.M., Sheehan, J.K., and Bishop, P.N. (2003). Light and X-ray scattering show decorin to be a dimer in solution. J Biol Chem 278, 1835318359. 
Scott, P.G., McEwan, P.A., Dodd, C.M., Bergmann, E.M., Bishop, P.N., and Bella, J. (2004). Crystal structure of the dimeric protein core of decorin, the archetypal small leucine-rich repeat proteoglycan. Proc Natl Acad Sci U S A 101, 15633-15638.

Sekizawa, J., Inouye, S., Halegoua, S., and Inouye, M. (1977). Precursors of major outer membrane proteins of Escherichia coli. Biochem Biophys Res Commun 77, 1126-1133.

Sernagor, E., and Mehta, V. (2001). The role of early neural activity in the maturation of turtle retinal function. J Anat 199, 375-383.

Setou, M., Nakagawa, T., Seog, D.H., and Hirokawa, N. (2000). Kinesin superfamily motor protein KIF17 and mLin-10 in NMDA receptor-containing vesicle transport. Science $288,1796-1802$.

Shen, Y., Heimel, J.A., Kamermans, M., Peachey, N.S., Gregg, R.G., and Nawy, S. (2009). A transient receptor potential-like channel mediates synaptic transmission in rod bipolar cells. J Neurosci 29, 6088-6093.

Sheng, H.Z., Fields, R.D., and Nelson, P.G. (1993). Specific regulation of immediate early genes by patterned neuronal activity. J Neurosci Res 35, 459-467.

Shieh, B.H., and Zhu, M.Y. (1996). Regulation of the TRP Ca2+ channel by INAD in Drosophila photoreceptors. Neuron 16, 991-998.

Shiells, R.A., and Falk, G. (1990). Glutamate receptors of rod bipolar cells are linked to a cyclic GMP cascade via a G-protein. Proc Biol Sci 242, 91-94.

Sidman, R.L. (1959). Histochemical studies on photoreceptor cells. Ann N Y Acad Sci $74,182-195$.

Sieving, P.A., Richards, J.E., Naarendorp, F., Bingham, E.L., Scott, K., and Alpern, M. (1995). Dark-light: model for nightblindness from the human rhodopsin Gly-90-->Asp mutation. Proc Natl Acad Sci U S A 92, 880-884.

Sjostrand, F.S. (1953). The ultrastructure of the outer segments of rods and cones of the eye as revealed by the electron microscope. J Cell Physiol 42, 15-44.

Sjostrand, F.S. (1958). Ultrastructure of retinal rod synapses of the guinea pig eye as revealed by three-dimensional reconstructions from serial sections. J Ultrastruct Res 2 , 122-170.

Smythe, E. (2002). Regulating the clathrin-coated vesicle cycle by AP2 subunit phosphorylation. Trends Cell Biol 12, 352-354.

Snellman, J., and Nawy, S. (2004). cGMP-dependent kinase regulates response sensitivity of the mouse on bipolar cell. J Neurosci 24, 6621-6628. 
Somasundaram, B., Norman, J.C., and Mahaut-Smith, M.P. (1995). Primaquine, an inhibitor of vesicular transport, blocks the calcium-release-activated current in rat megakaryocytes. Biochem J 309 ( Pt 3), 725-729.

Spitzer, N.C., Root, C.M., and Borodinsky, L.N. (2004). Orchestrating neuronal differentiation: patterns of $\mathrm{Ca} 2+$ spikes specify transmitter choice. Trends Neurosci 27, 415-421.

Stagljar, I. (2003). Finding partners: emerging protein interaction technologies applied to signaling networks. Sci STKE 2003, pe56.

Sterling, P. (1995). Vision. Tuning retinal circuits. Nature 377, 676-677.

Sterling, P. (1998). "Knocking out" a neural circuit. Neuron 21, 643-644.

Sterling, P., and Smith, R.G. (2004). Design for a binary synapse. Neuron 41, 313-315.

Stockton, R.A., and Slaughter, M.M. (1989). B-wave of the electroretinogram. A reflection of ON bipolar cell activity. J Gen Physiol 93, 101-122.

Strom, T.M., Nyakatura, G., Apfelstedt-Sylla, E., Hellebrand, H., Lorenz, B., Weber, B.H., Wutz, K., Gutwillinger, N., Ruther, K., Drescher, B., et al. (1998). An L-type calcium-channel gene mutated in incomplete X-linked congenital stationary night blindness. Nat Genet 19, 260-263.

Syed, M.M., Lee, S., Zheng, J., and Zhou, Z.J. (2004). Stage-dependent dynamics and modulation of spontaneous waves in the developing rabbit retina. J Physiol 560, 533-549.

Takebayashi, K., Takahashi, S., Yokota, C., Tsuda, H., Nakanishi, S., Asashima, M., and Kageyama, R. (1997). Conversion of ectoderm into a neural fate by ATH-3, a vertebrate basic helix-loop-helix gene homologous to Drosophila proneural gene atonal. EMBO J $16,384-395$.

Tanahashi, H., and Tabira, T. (1999). X11L2, a new member of the X11 protein family, interacts with Alzheimer's beta-amyloid precursor protein. Biochem Biophys Res Commun 255, 663-667.

Tang, Y., Tang, J., Chen, Z., Trost, C., Flockerzi, V., Li, M., Ramesh, V., and Zhu, M.X. (2000). Association of mammalian trp4 and phospholipase $C$ isozymes with a PDZ domain-containing protein, NHERF. J Biol Chem 275, 37559-37564.

Taylor, W.R., and Morgans, C. (1998). Localization and properties of voltage-gated calcium channels in cone photoreceptors of Tupaia belangeri. Vis Neurosci 15, 541-552.

Tian, N. (2004). Visual experience and maturation of retinal synaptic pathways. Vision Res 44, 3307-3316. 
Tian, N., and Copenhagen, D.R. (2001). Visual deprivation alters development of synaptic function in inner retina after eye opening. Neuron 32, 439-449.

Tian, N., and Slaughter, M.M. (1995a). Correlation of dynamic responses in the ON bipolar neuron and the b-wave of the electroretinogram. Vision Res 35, 1359-1364.

Tian, N., and Slaughter, M.M. (1995b). Functional properties of a metabotropic glutamate receptor at dendritic synapses of $\mathrm{ON}$ bipolar cells in the amphibian retina. Vis Neurosci 12, 755-765.

Tian, N., and Slaughter, M.M. (2003). Structure of glutamate analogs that activate the ON bipolar cell metabotropic glutamate receptor in vertebrate retina. Vis Neurosci 20, 231-240.

tom Dieck, S., and Brandstatter, J.H. (2006). Ribbon synapses of the retina. Cell Tissue Res 326, 339-346.

Tombola, F., Pathak, M.M., and Isacoff, E.Y. (2006). How does voltage open an ion channel? Annu Rev Cell Dev Biol 22, 23-52.

Torborg, C.L., and Feller, M.B. (2005). Spontaneous patterned retinal activity and the refinement of retinal projections. Prog Neurobiol 76, 213-235.

Trimarchi, J.M., Stadler, M.B., Roska, B., Billings, N., Sun, B., Bartch, B., and Cepko, C.L. (2007). Molecular heterogeneity of developing retinal ganglion and amacrine cells revealed through single cell gene expression profiling. J Comp Neurol 502, 1047-1065.

Turner, D.L., and Cepko, C.L. (1987). A common progenitor for neurons and glia persists in rat retina late in development. Nature 328, 131-136.

Turner, H., Fleig, A., Stokes, A., Kinet, J.P., and Penner, R. (2003). Discrimination of intracellular calcium store subcompartments using TRPV1 (transient receptor potential channel, vanilloid subfamily member 1) release channel activity. Biochem J 371, 341 350 .

Tusnady, G.E., and Simon, I. (1998). Principles governing amino acid composition of integral membrane proteins: application to topology prediction. J Mol Biol 283, 489-506.

Ullrich, B., Li, C., Zhang, J.Z., McMahon, H., Anderson, R.G., Geppert, M., and Sudhof, T.C. (1994). Functional properties of multiple synaptotagmins in brain. Neuron 13, 1281 1291.

Ullrich, B., and Sudhof, T.C. (1994). Distribution of synaptic markers in the retina: implications for synaptic vesicle traffic in ribbon synapses. J Physiol Paris 88, 249-257. 
Valente, P., Garcia-Sanz, N., Gomis, A., Fernandez-Carvajal, A., Fernandez-Ballester, G., Viana, F., Belmonte, C., and Ferrer-Montiel, A. (2008). Identification of molecular determinants of channel gating in the transient receptor potential box of vanilloid receptor I. FASEB J 22, 3298-3309.

Van Buren, J.J., Bhat, S., Rotello, R., Pauza, M.E., and Premkumar, L.S. (2005). Sensitization and translocation of TRPV1 by insulin and IGF-I. Mol Pain 1, 17.

van de Graaf, S.F., Chang, Q., Mensenkamp, A.R., Hoenderop, J.G., and Bindels, R.J. (2006a). Direct interaction with Rab1 la targets the epithelial Ca2+ channels TRPV5 and TRPV6 to the plasma membrane. Mol Cell Biol 26, 303-312.

van de Graaf, S.F., Hoenderop, J.G., and Bindels, R.J. (2006b). Regulation of TRPV5 and TRPV6 by associated proteins. Am J Physiol Renal Physiol 290, F1295-1302.

van de Graaf, S.F., Hoenderop, J.G., van der Kemp, A.W., Gisler, S.M., and Bindels, R.J. (2006c). Interaction of the epithelial Ca2+ channels TRPV5 and TRPV6 with the intestine- and kidney-enriched PDZ protein NHERF4. Pflugers Arch 452, 407-417.

Vardi, N. (1998). Alpha subunit of Go localizes in the dendritic tips of ON bipolar cells. J Comp Neurol 395, 43-52.

Vardi, N., Duvoisin, R., Wu, G., and Sterling, P. (2000). Localization of mGluR6 to dendrites of ON bipolar cells in primate retina. J Comp Neurol 423, 402-412.

Vardi, N., Matesic, D.F., Manning, D.R., Liebman, P.A., and Sterling, P. (1993). Identification of a G-protein in depolarizing rod bipolar cells. Vis Neurosci 10, 473-478.

Vardi, N., and Morigiwa, K. (1997). ON cone bipolar cells in rat express the metabotropic receptor mGluR6. Vis Neurosci 14, 789-794.

Venkatachalam, K., and Montell, C. (2007). TRP channels. Annu Rev Biochem 76, 387 417.

von Heijne, G. (1985a). Ribosome-SRP-signal sequence interactions. The relay helix hypothesis. FEBS Lett 190, 1-5.

von Heijne, G. (1985b). Signal sequences. The limits of variation. J Mol Biol 184, 99 105.

von Heijne, G. (1990a). Protein targeting signals. Curr Opin Cell Biol 2, 604-608. von Heijne, G. (1990b). The signal peptide. J Membr Biol 115, 195-201.

von Heijne, G. (2002). Bioinformatics of membrane proteins. Ernst Schering Res Found Workshop, 17-27. 
von Heijne, G., and Manoil, C. (1990). Membrane proteins: from sequence to structure. Protein Eng 4, 109-112.

Von Kriegstein, K., Schmitz, F., Link, E., and Sudhof, T.C. (1999). Distribution of synaptic vesicle proteins in the mammalian retina identifies obligatory and facultative components of ribbon synapses. Eur J Neurosci 11, 1335-1348.

Wachtmeister, L., and Dowling, J.E. (1978). The oscillatory potentials of the mudpuppy retina. Invest Ophthalmol Vis Sci 17, 1176-1188.

Wahlberg, J.M., and Spiess, M. (1997). Multiple determinants direct the orientation of signal-anchor proteins: the topogenic role of the hydrophobic signal domain. J Cell Biol $137,555-562$.

Wald, G. (1968). Molecular basis of visual excitation. Science 162, 230-239.

Walter, P., and Blobel, G. (1981). Translocation of proteins across the endoplasmic reticulum III. Signal recognition protein (SRP) causes signal sequence-dependent and site-specific arrest of chain elongation that is released by microsomal membranes. $\mathrm{J}$ Cell Biol 91, 557-561.

Walters, R.J., Kramer, R.H., and Nawy, S. (1998). Regulation of cGMP-dependent current in On bipolar cells by calcium/calmodulin-dependent kinase. Vis Neurosci 15, 257-261.

Wang, M., Bianchi, R., Chuang, S.C., Zhao, W., and Wong, R.K. (2007). Group I metabotropic glutamate receptor-dependent TRPC channel trafficking in hippocampal neurons. J Neurochem 101, 411-421.

Wassle, H., and Boycott, B.B. (1991). Functional architecture of the mammalian retina. Physiol Rev 71, 447-480.

Wassle, H., Yamashita, M., Greferath, U., Grunert, U., and Muller, F. (1991). The rod bipolar cell of the mammalian retina. Vis Neurosci 7, 99-112.

Watson, N., Linder, M.E., Druey, K.M., Kehrl, J.H., and Blumer, K.J. (1996). RGS family members: GTPase-activating proteins for heterotrimeric G-protein alpha-subunits. Nature 383, 172-175.

Watt, A.J., van Rossum, M.C., MacLeod, K.M., Nelson, S.B., and Turrigiano, G.G. (2000). Activity coregulates quantal AMPA and NMDA currents at neocortical synapses. Neuron 26, 659-670.

Weng, K., Lu, C., Daggett, L.P., Kuhn, R., Flor, P.J., Johnson, E.C., and Robinson, P.R. (1997). Functional coupling of a human retinal metabotropic glutamate receptor 
(hmGluR6) to bovine rod transducin and rat Go in an in vitro reconstitution system. J Biol Chem 272, 33100-33104.

Wetts, R., and Fraser, S.E. (1988). Multipotent precursors can give rise to all major cell types of the frog retina. Science $239,1142-1145$.

Whitfield, C.W., Benard, C., Barnes, T., Hekimi, S., and Kim, S.K. (1999). Basolateral localization of the Caenorhabditis elegans epidermal growth factor receptor in epithelial cells by the PDZ protein LIN-10. Mol Biol Cell 10, 2087-2100.

Windisch, J.M., Marksteiner, R., and Schneider, R. (1995). Nerve growth factor binding site on TrkA mapped to a single 24-amino acid leucine-rich motif. J Biol Chem 270, 28133-28138.

Wong, R.O., Chernjavsky, A., Smith, S.J., and Shatz, C.J. (1995). Early functional neural networks in the developing retina. Nature 374, 716-718.

Wong, R.O., Meister, M., and Shatz, C.J. (1993). Transient period of correlated bursting activity during development of the mammalian retina. Neuron $11,923-938$.

Wycisk, K.A., Budde, B., Feil, S., Skosyrski, S., Buzzi, F., Neidhardt, J., Glaus, E., Nurnberg, P., Ruether, K., and Berger, W. (2006a). Structural and functional abnormalities of retinal ribbon synapses due to Cacna2d4 mutation. Invest Ophthalmol Vis Sci 47, 3523-3530.

Wycisk, K.A., Zeitz, C., Feil, S., Wittmer, M., Forster, U., Neidhardt, J., Wissinger, B., Zrenner, E., Wilke, R., Kohl, S., et al. (2006b). Mutation in the auxiliary calcium-channel subunit CACNA2D4 causes autosomal recessive cone dystrophy. Am J Hum Genet 79, 973-977.

Xia, Y., Buja, L.M., and McMillin, J.B. (1996). Change in expression of heart carnitine palmitoyltransferase I isoforms with electrical stimulation of cultured rat neonatal cardiac myocytes. J Biol Chem 271, 12082-12087.

Xu, X.Z., Moebius, F., Gill, D.L., and Montell, C. (2001). Regulation of melastatin, a TRP-related protein, through interaction with a cytoplasmic isoform. Proc Natl Acad Sci U S A 98, 10692-10697.

Xu, X.Z., and Sternberg, P.W. (2003). A C. elegans sperm TRP protein required for sperm-egg interactions during fertilization. Cell 114, 285-297.

Yagi, T., and Macleish, P.R. (1994). Ionic conductances of monkey solitary cone inner segments. J Neurophysiol 71, 656-665. 
Yamashita, M., and Wassle, H. (1991). Responses of rod bipolar cells isolated from the rat retina to the glutamate agonist 2-amino-4-phosphonobutyric acid (APB). J Neurosci $11,2372-2382$.

Yao, Y., Ferrer-Montiel, A.V., Montal, M., and Tsien, R.Y. (1999). Activation of storeoperated $\mathrm{Ca} 2+$ current in Xenopus oocytes requires SNAP-25 but not a diffusible messenger. Cell 98, 475-485.

Yellen, G. (1998). The moving parts of voltage-gated ion channels. Q Rev Biophys 31, 239-295.

Young, R.W. (1985). Cell differentiation in the retina of the mouse. Anat Rec 212, 199205.

Yuste, R., Peinado, A., and Katz, L.C. (1992). Neuronal domains in developing neocortex. Science 257, 665-669.

Zeitz, C., Scherthan, H., Freier, S., Feil, S., Suckow, V., Schweiger, S., and Berger, W. (2003). NYX (nyctalopin on chromosome X), the gene mutated in congenital stationary night blindness, encodes a cell surface protein. Invest Ophthalmol Vis Sci 44, 4184-4191. Zerial, M., and McBride, H. (2001). Rab proteins as membrane organizers. Nat Rev Mol Cell Biol 2, 107-117.

Zhang, J., Jeffrey, B.G., Morgans, C.W., Burke, N.S., Haley, T.L., Duvoisin, R.M., and Brown, R.L. (2010). RGS7 and -11 complexes accelerate the ON-bipolar cell light response. Invest Ophthalmol Vis Sci 51, 1121-1129.

Zhang, X., Wensel, T.G., and Kraft, T.W. (2003). GTPase regulators and photoresponses in cones of the eastern chipmunk. J Neurosci 23, 1287-1297.

Zhou, Y., Deneris, E., and Zigmond, R.E. (2001). Nicotinic acetylcholine receptor subunit proteins alpha7 and beta4 decrease in the superior cervical ganglion after axotomy. J Neurobiol 46, 178-192. 


\section{APPENDIX I \\ GENES THAT ARE MODULATED BY LOSS OF NYCTALOPIN}

\section{INTRODUCTION}

Retinogenesis is initiated from a common population of multipotent retinal progenitor cells (Turner et al., 1987; Wetts et al., 1988). In vertebrate retina, neuronal differentiation follows a fixed chronological order and is initiated from the central optic cup and progresses concentrically in a wave-like manner until it reaches the periphery of the retina (Prada et al., 1991). Retinal ganglion cells and horizontal cells are the first neurons to differentiate. They are followed in an overlapping fashion by the cone photoreceptors, the amacrine cells, the rod photoreceptors and finally bipolar cells (Sidman, 1959; Young, 1985; Marquardt and Gruss, 2002). However, synaptic connections between these different neuronal cell types do not exactly follow the same timing (Nishimura \& Rakic, 1987). Synapses between bipolar cells and amacrine cells form first. They are followed by synapse formation between the photoreceptor and the horizontal cells in the OPL. Synapses between the bipolar cell and photoreceptor and bipolar cell and ganglion cell are the last to appear. In mice, synaptogenesis at ribbon and conventional synapses in the OPL is complete by postnatal day 15 (P15) (Blanks, 1974; Fisher, 1979) and is separated into three different stages: synapse formation, maturation and maintenance (Mey and Thanos, 1992; Lillien et al., 1994; Hendrickson et 
al., 1996). The first stage of synaptogenesis is under genetic control and involves the apposition of processes that will become dendrites and axons (Harris, 1981, Harris and Holt, 1990). The second stage is the formation of synapses. The initial contact results in the formation and assembly of pre and postsynaptic machinery for synaptic activity (Harris, 1981, Harris and Holt, 1990). The third step is synapse maintenance. The mechanism that guides synaptic assembly and maintenance in the retina is poorly understood, however spontaneous activity in the form of retinal waves is involved in synapse maturation and maintenance.

Spontaneous activity in the form of retinal waves is a burst of propagating action potentials and is a general mechanism used by the developing nervous system to assemble and refine circuitry (Masland, 1977; Galli and Maffei, 1988; Meister et al., 1991; Wong et al., 1993; Feller et al., 1999). Retinal waves are involved in the formation and sculpting of synapses in the retina, lateral geniculate nucleus (LGN), cochlea, spinal cord, hippocampus, and the cortex (Yuste et al., 1992; Wong et al., 1995; Schwartz et al., 1998; Goldberg et al., 2002; Leinekugel, 2003; Mohajerani and Cherubini 2006; Gonzalez-Islas and Wenner, 2006; Nicol et al., 2007; Hanson et al., 2008). The theory regarding retinal development is that retinal waves contribute to synaptogenesis by regulating the expression of guidance molecules (see review Torborg and Feller, 2005). Studies done in frog and chick spinal neurons have demonstrated that spontaneous waves can regulate transcriptional and translational processes (Watt et al., 2000; Borodinsky et al., 2004; Hanson and Lanmesser, 2004; Spitzer et al., 2004). In normal conditions, infrequent depolarization induced by retinal waves may generate sufficient calcium influx to stimulate transcription (Dolmetsch et al., 1998). Such elevations in calcium have been 
shown to induce the transcription of immediate early genes and activate the promoters of specific genes (Dash et al., 1991; Sheng et al., 1993; Xia et al., 1996; Fields et al., 1997). In lymphoid cells, transcription has been shown to be influenced by the duration, frequency and the amplitude of the calcium transients (Dolmetsch et al., 1997, 1998; Li et al., 1998).

In the retina, spontaneous activity in the form of retinal waves persists throughout the development of the retina (Sernagor et al., 2001). As retinal waves change, the circuitry in the retina also changes (see review Torborg and Feller, 2005). During the development of the retina, three different retinal waves emerge and these are defined based on their mechanism of mediation. Stage I waves begins at embryonic day 16 (E16) and continue till postnatal day zero (P0). They are mediated mainly by gap junctions and occur before the formation of conventional synapses (Syed et al., 2004). Stage I waves can be blocked by gap junction blockers such as glycyrrhetinic acid or carbenoxylone (Wong et al., 1998). Stage II waves occur between P0 and P10 and are mediated by nicotinic acetylcholine receptors (Bansal et al., 2000). These waves can be blocked by agonists for acetylcholine receptors such as epibatidine or curare (Feller et al., 1996; Penn et al., 1998). They can also be blocked genetically by using knockout mice that lack the $\beta 2$ subunit of the acetylcholine receptors (Feller et al., 1996; Bansal et al., 2000). Stage III waves occur between P10 to P14 and are mediated by glutamate receptors (GluRs) (Feller et al. 1996; Bansal et al. 2000; Zhou, 2001; Demas et al. 2003; Blankenship et al., 2009; Feller and Blankenship, 2008). Stage III waves are blocked by antagonists for ionotropic glutamate receptors (NBQX, APV) and agonist for the 
metabotropic glutamate receptors (L-APB) (Wong et al., 1998; Bansal et al., 2000; Chapman et al., 2000).

In mice the transition from the stage II to stage III waves is correlated with the time when light responses are first detected in ganglion cell (Fisher, 1979; Miller et al., 1999; Demas et al., 2003; Tian and Copenhagen, 2003; Morgan et al., 2008). Knockout mice lacking the vesicular glutamate transporter type 1 (VGLUT1) do not have stage III waves but continue to exhibit stage II waves. VGLUT1 is localized to the pre-synaptic side of the OPL at the photoreceptor terminal and is responsible for loading glutamate into photoreceptor synaptic vesicles. The loss of VGLUT1 means that glutamate release is abrogated in this mouse suggesting a role of glutamate release from the Photoreceptor in the mediation of stage II waves.

Several presynaptic mutants (Cacnalf ${ }^{\text {nob2 }}$, Capb $^{-/}$, Cacnb2 $^{-/}$, Cacna4d2 $2^{-1}$ ) have been characterized anatomically and morphologically, none have been characterized for retinal waves. The characterized the Cacnal $f^{\text {nob2 }}$ mouse mutant (Chang et al., 2006) has a defect cause by an insertion of a transposable element in exon 2. Cacnalf encodes the alpha1 subunit of the voltage dependent calcium channel, which means glutamate release into the OPL is abrogated in this mouse too. Although wave activity has not been studied in Cacnalf $f^{n o b 2}$ mouse, this mouse is expected to continue to exhibit stage II retinal waves. In addition voltage dependent calcium channels have been shown to be involved in gene expression (Cartin et al., 2000). The synaptic structure is disorganized in this mouse with the dendrites of both bipolar cell and horizontal cell ectopically extending into the ONL (Chang et al; 2006). In the initial small scale qPCR experiment, this mouse was used to determine the effect of pre-synaptic defects on genes expressed in the OPL. 
In $N y x^{n o b}$ mice, signaling is absent from depolarizing bipolar cells and stage III retinal waves and abnormal spontaneous activity is maintained into adulthood (Demas et al., 2006). The pattern of the retinal waves also is changed from infrequent bursting to frequent bursting (Demas et al., 2006). However, the morphology of the $N y x^{n o b}$ retina is normal at both electron and light microscope levels (Pardue et al., 2001; Ball et al., 2003; Gregg et al., 2007). Immunohistochemical analysis of the $N y x^{n o b}$ retina showed that PKC, TrKB, PSD-95, bassoon, Dystrophin, Cacna1f $(\alpha 1 \mathrm{~F})$, Grm6 and $\mathrm{G} \alpha \mathrm{O}$ are all localized properly (Ball et al., 2003).

As neurons in the retina mature into morphologically distinct cell types, the gene expression profile and protein composition for each cell type also changes (Greferath et al., 1990; Berrebi et al., 1991; Euler and Wassle, 1995; Takebayashi et al., 1997; Vardi and Morigiwa, 1997; Koulen et al., 1998; Fletcher et al., 1998; Vardi, 1998; Baas et al., 2000; Haeseleer et al., 2000; Chow et al., 2001; Ohtoshi et al., 2001; Haverkamp et al., 2003a,b; Huang et al., 2003). Among the molecules expressed specifically in different neuronal cell types are neurotransmitter receptors, signal transduction proteins, calcium binding proteins, and transcription factors. For example, specific splice forms of $\mathrm{GaO}$

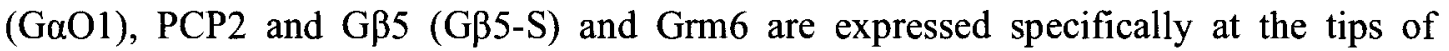
Bipolar cells (Nomura et al., 1994; Vardi et al., 2000; Dhingra et al., 2002; Huang et al., 2003). Studies of synaptogenesis have relied on the analysis of molecular makers to describe the development of normal and disease retinas. The temporal expression pattern of genes has been used to identify novel molecular markers for bipolar cells (Trimarchi et al., 2007; Kim et al., 2008). 
Thus an important question is, whether there is a correlation between abnormal retinal waves in the $N y x^{n o b}$ retinas and altered gene expression patterns. More importantly, if gene expression is altered, can the changes be used to identify novel genes involved in the Grm6 signaling cascade? To answer this question, I used quantitative real time PCR (qPCR) to analyze the expression levels of several genes expressed in the OPL during development. An initial results indicate that the expression level of several genes differ at postnatal day $13(\mathrm{P} 13)$ between $N y x^{n o b}$ and the control retina. To examine expression more extensively, microarray analysis were used to examine the expression level of 45,000 transcripts in $C 57 B L / 6 J$ and $N y x^{n o b}$ retinas. Sixteen genes that were significantly down or up regulated were analyzed further using qPCR. The expression of seven other genes (Plcd1, Capb2, GPC, Gde, Arhgap12, Frmd3 and Fam123a) was analyzed because they were shown to be bipolar specific genes (Nakagima et al., 2009). I also analyzed the expression level of Apbal (Mint1), and Trpml, because they interact with nyctalopin in my yeast two hybrid experiments (Chapter III). Quantitative real time PCR confirmed the array data for three of the sixteen genes studied. In addition, the expression of Apbal, Trpm1, GPC6 and Fam123a were significantly decreased in Nyx ${ }^{n o b}$ compared to $\mathrm{C} 57 \mathrm{BL} / 6 \mathrm{~J}$ retinas.

\section{METHODS}

\section{RNA extraction and cDNA synthesis}

These experiments were conducted in accordance to the rules set by ARVO for the use of animals in Ophthalmology and Vision Research and approved by the institutional Animal care and Use Committee at the University of Louisville. Adult mice were euthanized by $\mathrm{CO}_{2}$ inhalation. Mice less than two weeks old were anaesthized on 
ice first, decapitated and the eye removed. The eye were washed in 1xPBX before the retina was removed and stored in RNAlater (Ambion, Austin, TX) for 24 hours at $4^{\circ} \mathrm{C}$.

Total RNA was extracted using Trizol (Invitrogen Corporation, Carlsbad, CA) following the manufacturers' instructions. Before cDNA synthesis, the quality of the RNA was analyzed using the Nanodrop. To prepare cDNA, 500ng of total RNA was combined with $50 \mathrm{ng}$ of random hexamers, $10 \mathrm{mM} \mathrm{dNTP}$ in a final volume of $12 \mu \mathrm{l}$ and the mixture incubated at $65^{\circ} \mathrm{C}$ for 5 minutes and then incubated on ice for 2 minutes. SuperScript Reverse Transcriptase (200U), RNaseOUT $^{\mathrm{TM}}$ ribonuclease inhibitor (40U), 5X First strand buffer $(4 \mu \mathrm{l})$, and $0.01 \mathrm{M}$ of DTT $(2 \mu \mathrm{l})$ were added to each tube. Samples were incubated at $25^{\circ} \mathrm{C}$ for 5 minutes, followed by 1 -hour incubation at $50^{\circ} \mathrm{C}$. The reaction was terminated by incubation at $70^{\circ} \mathrm{C}$ for 15 minutes.

The relative quantity of selected genes was determined using the $\Delta \Delta \mathrm{CT}$ method. For bipolar specific genes the quantity of each gene was determined using qPCR and standard curve method. A standard cDNA pool was created by mixing $30 \mu l$ of cDNA from each time point analyzed (i.e. P1, P3, P5, P7, P13, P21). The pooled standard was used to generate a dilution series, $1 / 6,1 / 36$ and $1 / 216$ that were used to generate the standard curves.

To check for the efficiency of each primer set, the slope of each standard curve was determined. A quality primer set has a slope of approximately $\sim-3.34$. Statistical analysis was done using $\mathbf{t}$-Test.

\section{Quantitative PCR (qPCR)}

Primers were designed against portions of each gene's open reading frame (ORF) using Primer 3 software (http://frodo.wi.mit.edu/cgi-bin/primer3/primer3.cgi). Special care was 
taken to design primers that spanned introns to eliminate the chance of amplifying genomic DNA. Melting curves were used to ensure there was no primer dimer formation. Quantitative PCR was performed in $10 \mu 1$ reaction volumes containing: $2.0 \mu 1$ of cDNA, $0.5 \mu l$ gene-specific primer pairs $(20 \mu \mathrm{M})$, and 5.0 $\mu$ l Power SYBR Green PCR master mix (Applied Biosystem, Foster City, CA) or Taqman PCR master mix (Applied Biosystem, Carlsbad, CA). Real Time PCR amplification was performed on the Applied Biosystem (AB) 7900HT Fast Real-Time PCR system in accordance with the manufacturer protocol. At the end of the PCR, data were analyzed using the $\triangle \Delta C T$ method to determine the relative expression level of each gene. In the case of determining bipolar specific genes, the quantity of each gene was determined using the standard curve method.

\section{Microarray Analysis of gene expression}

RNA isolation, array hybridization and processing were done by Dr. Eldon Geisert at the University of Tennessee, Memphis, TN.

The transcriptome of the mouse retina was profiled using the Illumina Sentrix Mouse WG-6 V2 Expression BeadChips. These arrays interrogate $\sim 45,000$ transcripts. Three independent total RNA samples were repeated from 2 retinas each from $C 57 \mathrm{BL} / 6 \mathrm{~J}$ and $N y x^{\text {moth }}$. The retinas were homogenized and total RNA extracted with RNA-Stat-60 as described by the manufacturer (Tel-Test, Friendswood, TX). The quality and purity of RNA was assessed using an Agilent Bioanalyzer 2100 system (housed at UTHSC Molecular Research Center) to confirm the RNA Integrity number of 9.8-10.0. Labeled cRNA was produced using Illumina TotalPrep RNA Amplification Kit (Ambion, Austin,

TX). The cRNA for each sample was then hybridized to an Illumina Sentrix ${ }^{R}$ Mouse- 6 V2 BeadChip (Illumina, San Diego, CA). Quality control analysis of the raw image data 
was performed using the Illumina BeadStudio software. MIAME standards were used for all microarray data. Rank invariant normalization with BeadStudio software was used to calculate the relative expression values and, the data were globally normalized across all samples using the formula 2 (z-score of $\log 2$ [intensity]) +8 . All calculations and comparisons were done by Dr. Eldon Geisert. Comparisons between the $C 57 B L / 6 J$ mice and the $N y x^{\text {noh }}$ mutant mice were made using FileMaker Pro software.

\section{RESULTS}

\section{Transient alteration of expression of synaptic genes at P13}

To determine the function of nyctalopin in the retina, Ball et al. (2003) and Pardue et al. (1998) examined the $N y x^{n o b}$ retina using immunohistochemical analyses. These studies showed that the $N y x^{n o b}$ retina is normal at both the light and electron microscopic level. At the functional level, the loss of nyctalopin results in abnormal retinal activity in the form of continuous retinal waves (Demas et al., 2006). In normal mice, retinal waves have been shown to cause calcium transients, which in turn could affect gene expression (Dolmetsch et al., 1998). To assess the validity of this hypothesis, I first analyzed the expression of a small number of the genes that were known to be expressed in the OPL (Figure 30). These genes are: Nyx, Grm6, Cancnalf, Cacnald, and Cacnb2. Grm6 and Nyx are bipolar specific genes while Cancnalf, Cacnald, and Cacnb2 are expressed at the terminals of photoreceptors (Kim et al., 2008; Chang et al., 2006; Morgans et al., 2005).

The expression of these genes was analyzed in the C57BL/6J, $N y x^{n o b}$ and Cacnalf $f^{n o b 2}$ mice. The Cacnalf $f^{\text {ob2 } 2}$ phenotype is caused by a defect in the Cacnalf gene that encodes the alphal subunit of voltage dependent calcium channels that are expressed 
Figure 30. Developmental expression of genes expressed in the OPL. Quantitative real time PCR (qPCR) result showing the expression level of genes in $C 57 B L / 6 J, N y x^{n o b}$ and Cacnalf ${ }^{\text {nob2 }}$ during retinal development of the retina. Expression levels were normalized to 18S RNA and calibrated to $C 57 B L / 6 J$ adult (3month old). A total of three biological replicates were done for each experimental data point. Data are mean $+/-\mathrm{SE}$. 
Nyx

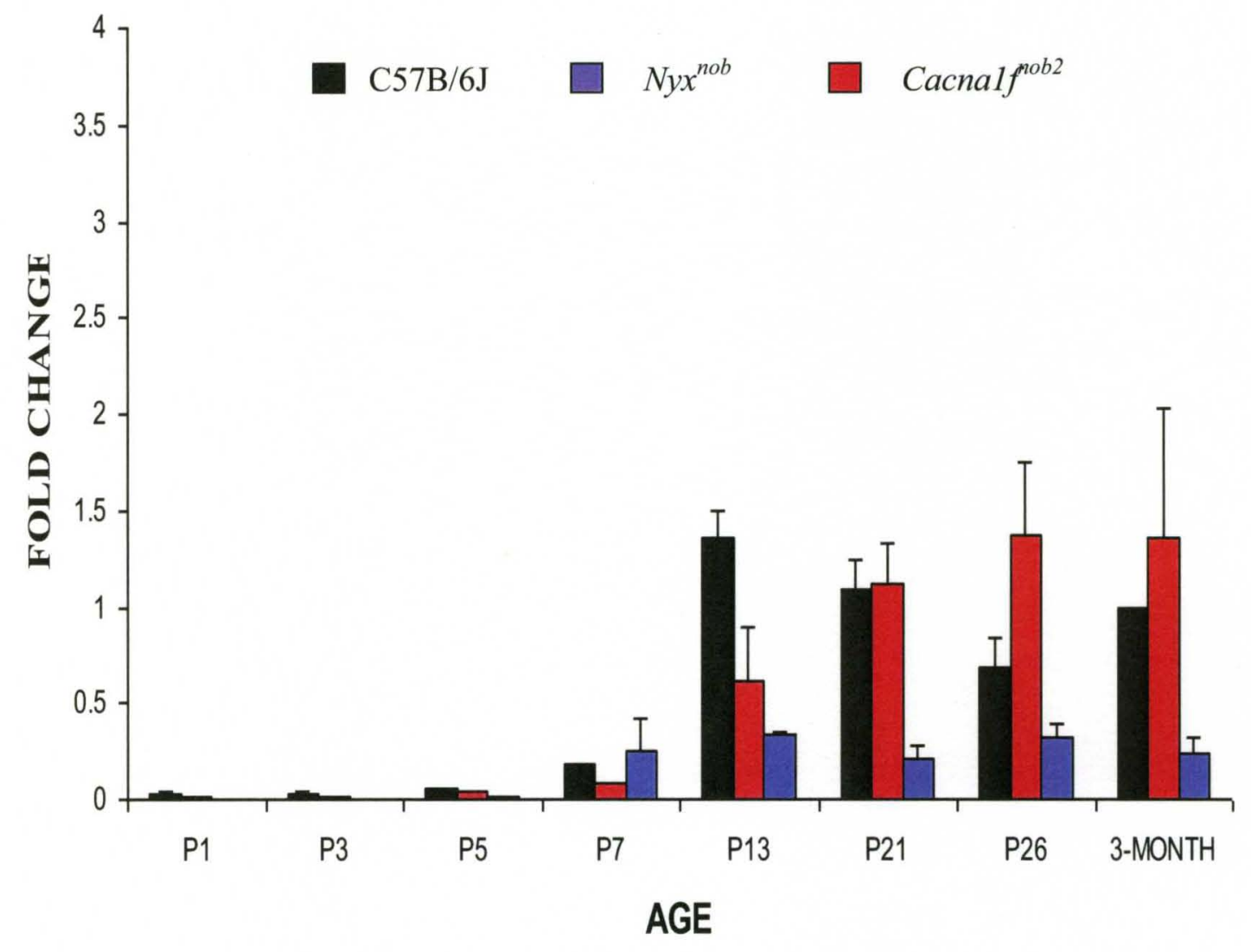


Grm6

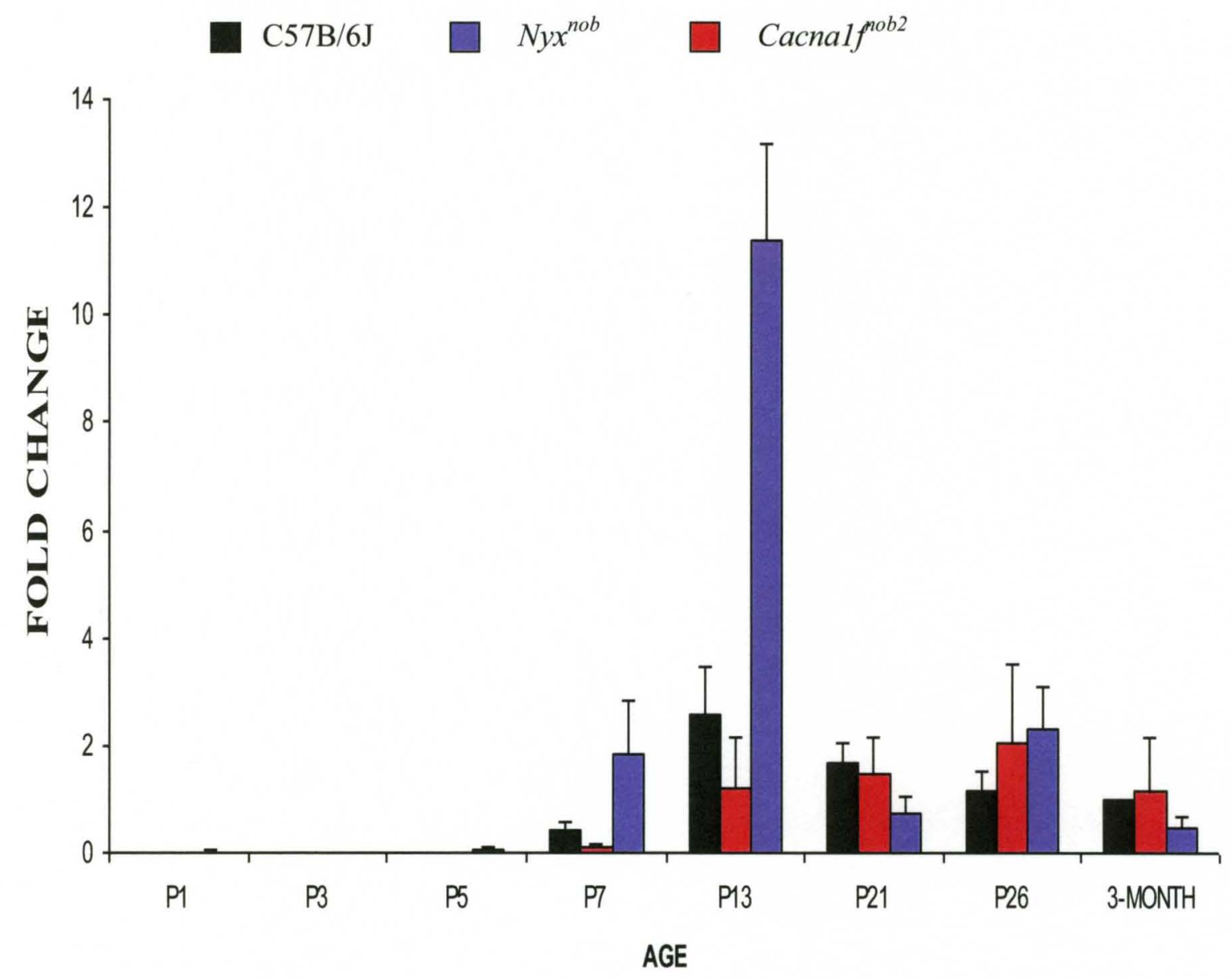


Cacnalf

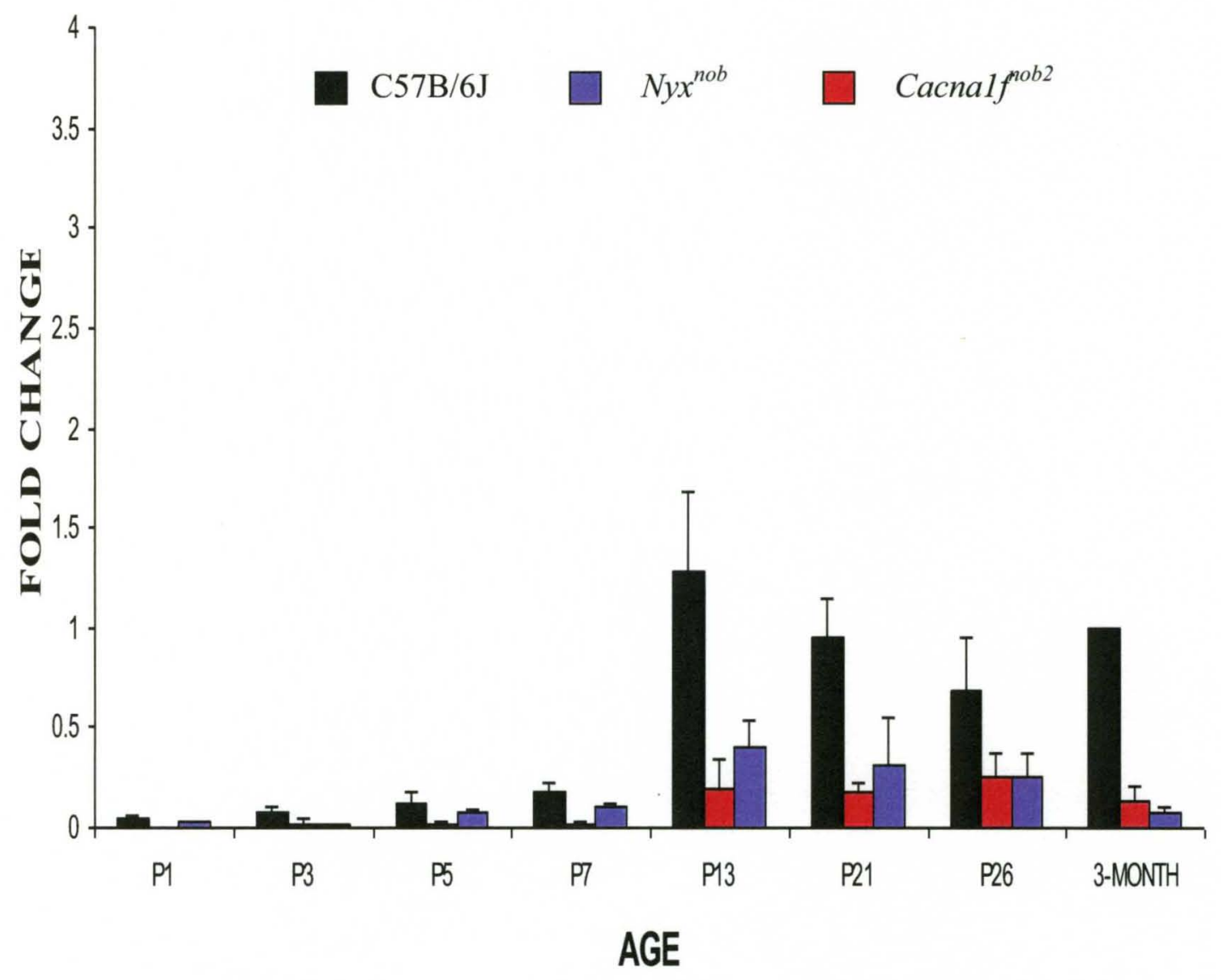




\section{Cacnald}

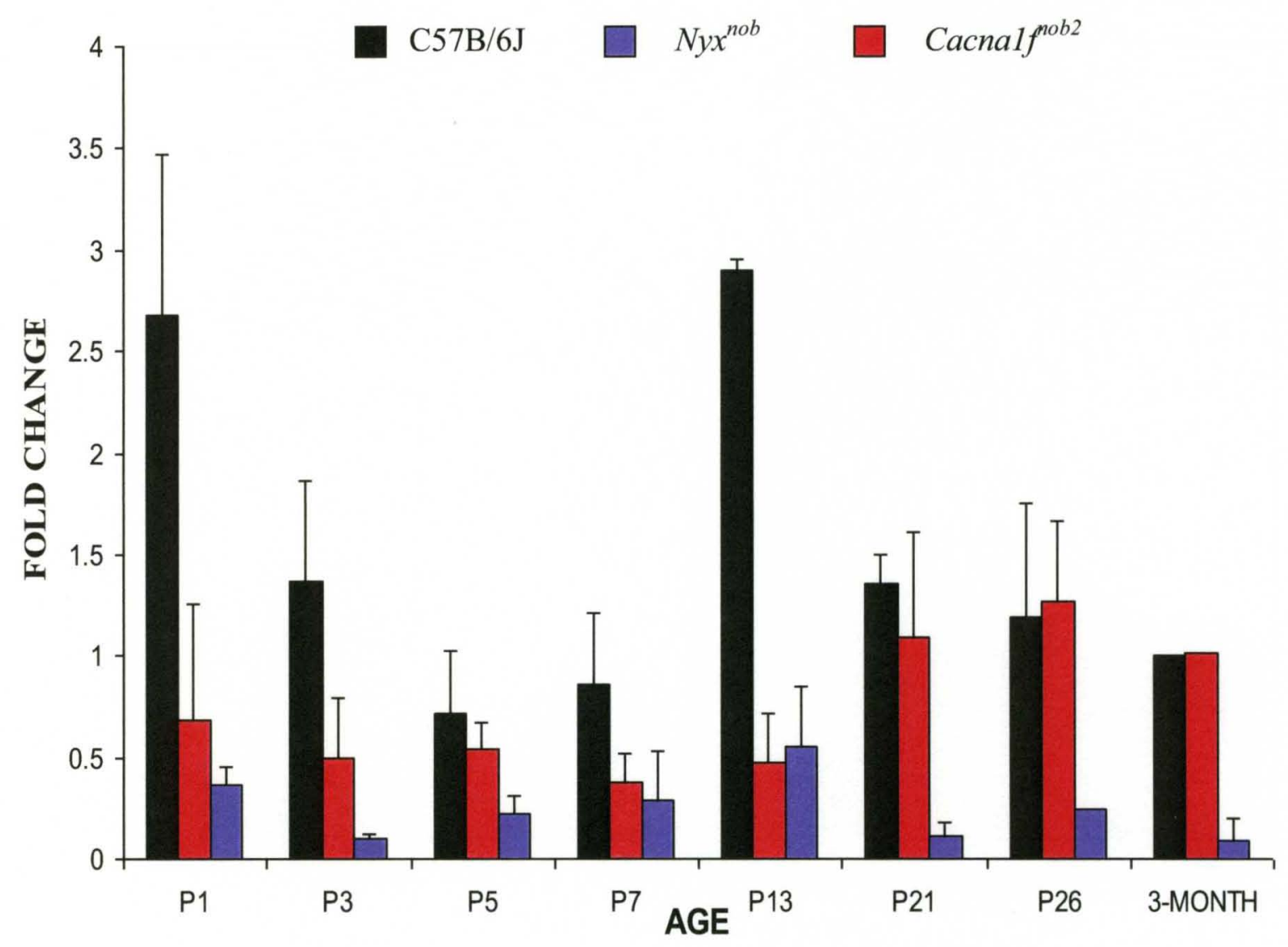




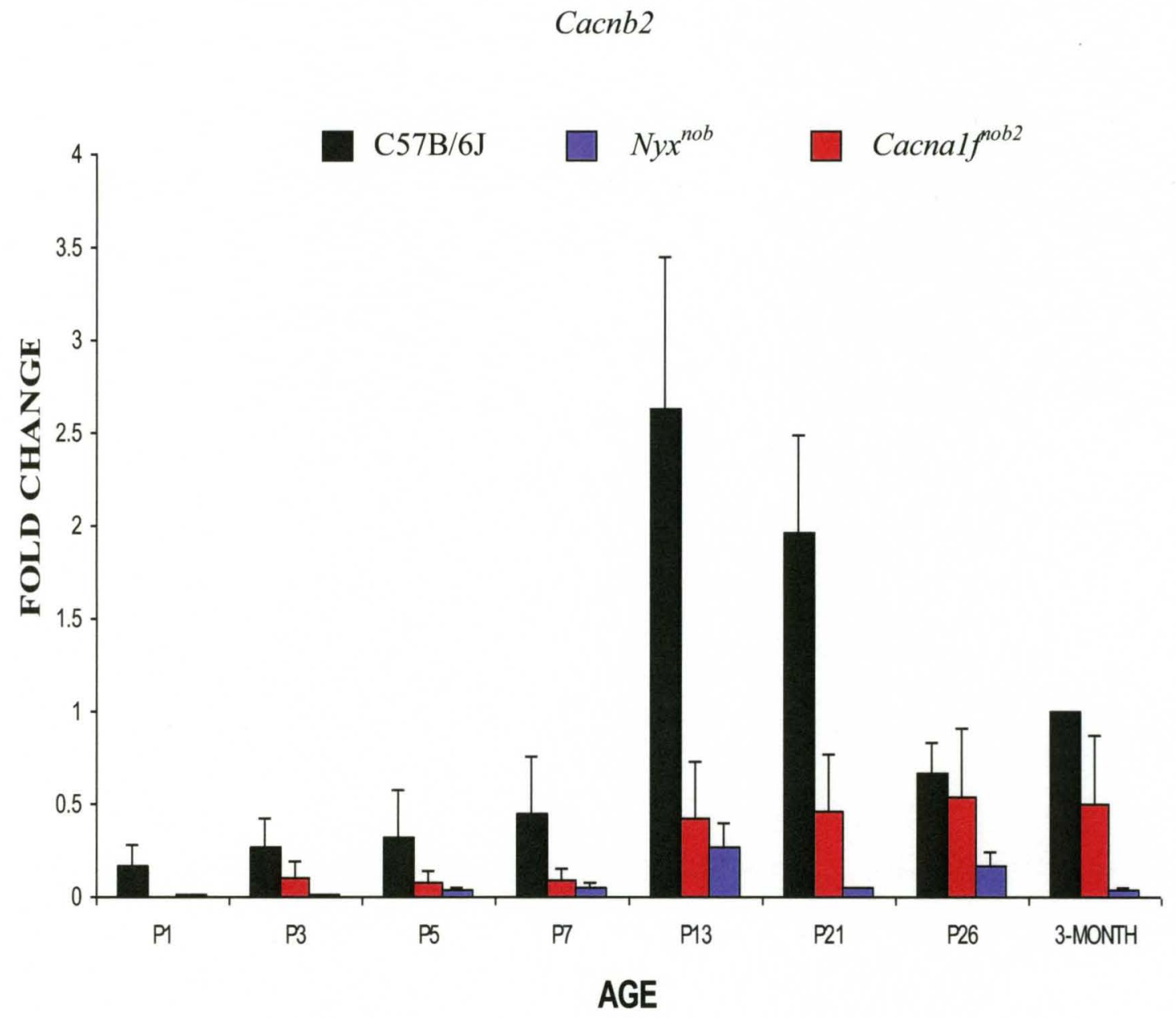


in both photoreceptors and bipolar cells. Calcium entry into voltage dependent channels results in glutamate release into the OPL and IPL.

In $C 57 B L / 6 J$ retinas the expression of all genes Nyx, Grm6, Cacnalf, Cacnald, and Cacnb2 peaked at eye opening (P11-13), which is correlated with the peak of retinal synapse maturation (Figure 30). In $N y x^{n o b}$ retina, the expression of Grm6 increased 4-fold compared to wild type at P13. In adult $N y x^{n o b}$ mice the level of Grm6 went down. In contrast, the expression of all VDCC subunits (i.e. Cacnalf, Cacnald, and Cacnb2) decreased in $N y x^{n o b}$ retina at every time point compared to $C 57 B L / 6 J$. The level of Cacnalf mRNA in $\mathrm{Nyx}^{\text {nob }}$ mice is almost at the same level as in Cacnalf ${ }^{\text {nob2 }}$ mice at every time point. Cacnal ${ }^{\text {nob2 }}$ mice show a delay of 8 days in the peak expression of all genes analyzed compared to $C 57 B L / 6 J$. In addition, there is a transient decrease in the level of all genes around P13 in Cacnalf ${ }^{\text {nob2 } 2}$ compared to C57BL/6J. The expression profile of Nyx mRNA changes in Cacnalf ${ }^{\text {nob2 }}$ mice. There is a steady increase in levels of Nyx mRNA in Cacnalf ${ }^{n o b 2}$ from P5 to adult (Figure 30).

Abnormalities that result in defective photoreceptor to bipolar cell signaling have differential effects on the expression levels of genes coding for synaptic proteins. In Cacnalf ${ }^{\text {pob2 }}$ mouse, peak expression of all genes is delayed. In $N y x^{\text {nob }}$ mouse, the expression of presynaptic proteins is decreased. In both $N y x^{n o b}$ and Cacnalf $f^{n o b 2}$ the alterations in expression patterns are transient and are most significant around the time of eye opening (P13), when synaptogenesis is maximal. Thus microarray analysis was done at P13 on $\mathrm{Nyx}^{\text {nob }}$ versus $\mathrm{C} 57 \mathrm{BL} / 6 \mathrm{~J}$ mice to estimate the expression level of $\sim 45000$ transcripts. 
Figure 31. Scatter plot showing variation in gene expression between $N y x^{n o b}$ and C57BL/6J at postnatal day 13. Each spot indicates the level of expression of a transcript. For many genes there are multiple probes and the expression level for each may be different. Two examples $C c l 27$ and Slit 2 are shown. 


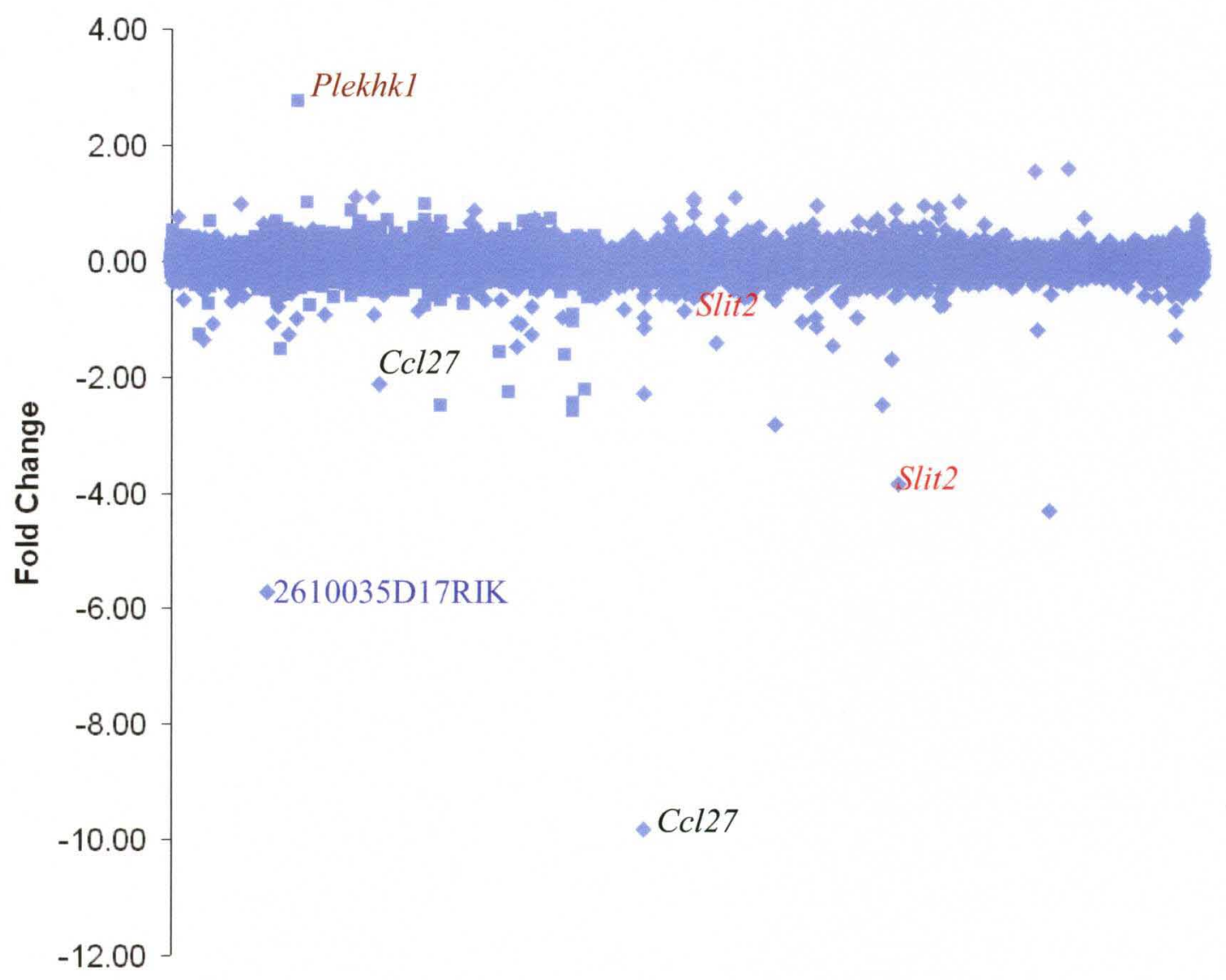


Table 4. Genes selected for confirmation by qPCR

\begin{tabular}{clccl}
\hline & Gene & Fold Change & P-value & Cominents \\
\hline 1 & LOC381345 & 2.60 & 0.021 & Transcript not available at ensembl \\
2 & Kcnb1 & 2.04 & 0.037 & Good p-value/fold change \\
3 & 2210419D22Rik & 1.98 & 0.042 & Transcript not available at ensembl \\
4 & Igsf9 & 1.91 & 0.002 & Good p-walue/fold change \\
5 & Abhd1 & 1.86 & 0.006 & Good p-walue/fold change \\
6 & DKK3 & 1.70 & 0.001 & Good p-walue/fold change \\
7 & Capb2 & -1.23 & 0.430 & Bipolar specific gene \\
8 & Frmd3 & 1.00 & 0.985 & Bipolar specific gene \\
9 & Plcd1 & -1.45 & 0.040 & Good p-value/fold change/bipolar specific \\
10 & GPC & -1.32 & 0.042 & Bipolar specific gene \\
11 & Fam123a & -1.00 & 0.042 & Bipolar specific gene \\
12 & Apba1 & 1.16 & 0.364 & Interacted with nyctalopin in MYTH \\
13 & Trpm1 & -1.25 & 0.205 & Interacted with nyctalopin in MYTH \\
14 & 2610528A15Rik & -2.07 & 0.017 & Good p-value/fold change \\
15 & 1700027L20Rik & -2.09 & 0.003 & Good p-value/fold change \\
16 & Arhgap 12 & -2.10 & 0.005 & Good p-value/fold change/bipolar specific \\
17 & Ccl21c & -2.16 & 0.007 & Good p-value/fold change \\
18 & LOC269531 & -2.17 & 0.024 & Transcript not available at ensembl \\
19 & Pex19 & -2.53 & 0.037 & Good p-value/fold change \\
20 & Thumpd1 & -2.57 & 0.001 & Good p-value/fold change \\
21 & Vwf & -2.62 & 0.001 & Good p-value/fold change \\
22 & Gsg1 & -2.68 & 0.041 & Good p-value/fold change \\
23 & $6330418 B 08 R i k$ & -3.13 & 0.019 & Transcript not available at ensembl \\
24 & Zfp238 & -3.22 & 0.020 & Good p-value/fold change \\
25 & Gpr125 & -3.47 & 0.002 & Good p-value/fold change \\
26 & Slit2 & -3.50 & 0.001 & Good p-value/fold change \\
27 & LOC331139 & -5.29 & 0.033 & Transcript not awailable at ensembl \\
28 & $2610035 D 17 R i k$ & -6.72 & 0.001 & Transcript not available at ensembl \\
29 & Cc127 & -10.81 & 0.024 & Good p-value/fold change \\
30 & Gde & 1.00 & 0.830 & Bipolar specific gene \\
\hline & & & &
\end{tabular}

Transcript is not available means qPCR was not performed on these genes. 


\section{Microarray analysis of gene expression on $\mathrm{C} 57 \mathrm{~B} / 6 \mathrm{~J}$ and $\mathrm{Nyx}{ }^{\text {nob }}$ retinas}

To estimate the expression level of genes in the retina at postnatal day 13 (P13), we used Illumina Mouse WG-6 V2 microarrays. The arrays interrogated $\sim 45000$ transcripts. For many genes, there are multiple probes and the fold change for each probe often does not agree. For example one probe estimated the expression of Slit 2 at -0.66 while another estimated it at -2.29 , a 1.63 fold difference between the two probes (Figure 31). For $C c l 27$, the expression was estimated by one probe at -2.5 while another probe estimated it at -9.81 , a 7.31 fold difference (Figure 31). This makes Illumina Mouse WG$6 \mathrm{~V} 2$ array estimation of gene expression useful, however, the data require careful analysis to ensure that reliable gene expression levels are obtained.

As can be seen in Figure 31, the majority of the genes interrogated in the array analysis did not change. To select genes for further analysis, I used several criteria. First, I chose genes that showed a significant difference $(\mathrm{p}<0.05)$ in expression (fold change of 1.5 or greater). Twenty two genes had a decrease or increase of more than 1.5 fold (Figure 32). I also considered functional relevance to signaling in the retina (DBC cell) and in some cases genes were selected because they interacted with nyctalopin in the yeast two hybrid screens (Apbal and Trpml). In addition, I chose genes because they have been shown in the literature to be bipolar specific genes (Plcdl, Capb2, GPC, Gde, Arhgap12, Frmd3 and Fam123a) (Figure 32) (Nakagima et al., 2009). PLekhk1 was not selected because it has a high $p$-value $(\mathrm{p}=0.2)$ for one probe (Figure 31$)$. The fold change for the other probes are less than 1.5 fold. Six other transcripts (LOC381345, 2210419D22RIK, LOC296531, 6330418B08RIK, LOC331139, 2610035D17RIK) were 
Figure 32. Genes with altered expression levels in $N y x^{n o b}$ mice. The expression level of 45,000 transcripts in $\mathrm{C} 57 \mathrm{BL} / 6 \mathrm{~J}$ and $N y x^{n o b}$ retinas were analyzed using Illumina bead array. The genes shown here were selected based on significant difference in expression $(\mathrm{p}<0.05)$, fold change $(>1.5)$, bipolar specific expression and relevancy to signaling in the depolarizing bipolar cells. Apbal and Trpml were selected because they interacted with nyctalopin in the yeast two hybrid experiments. 


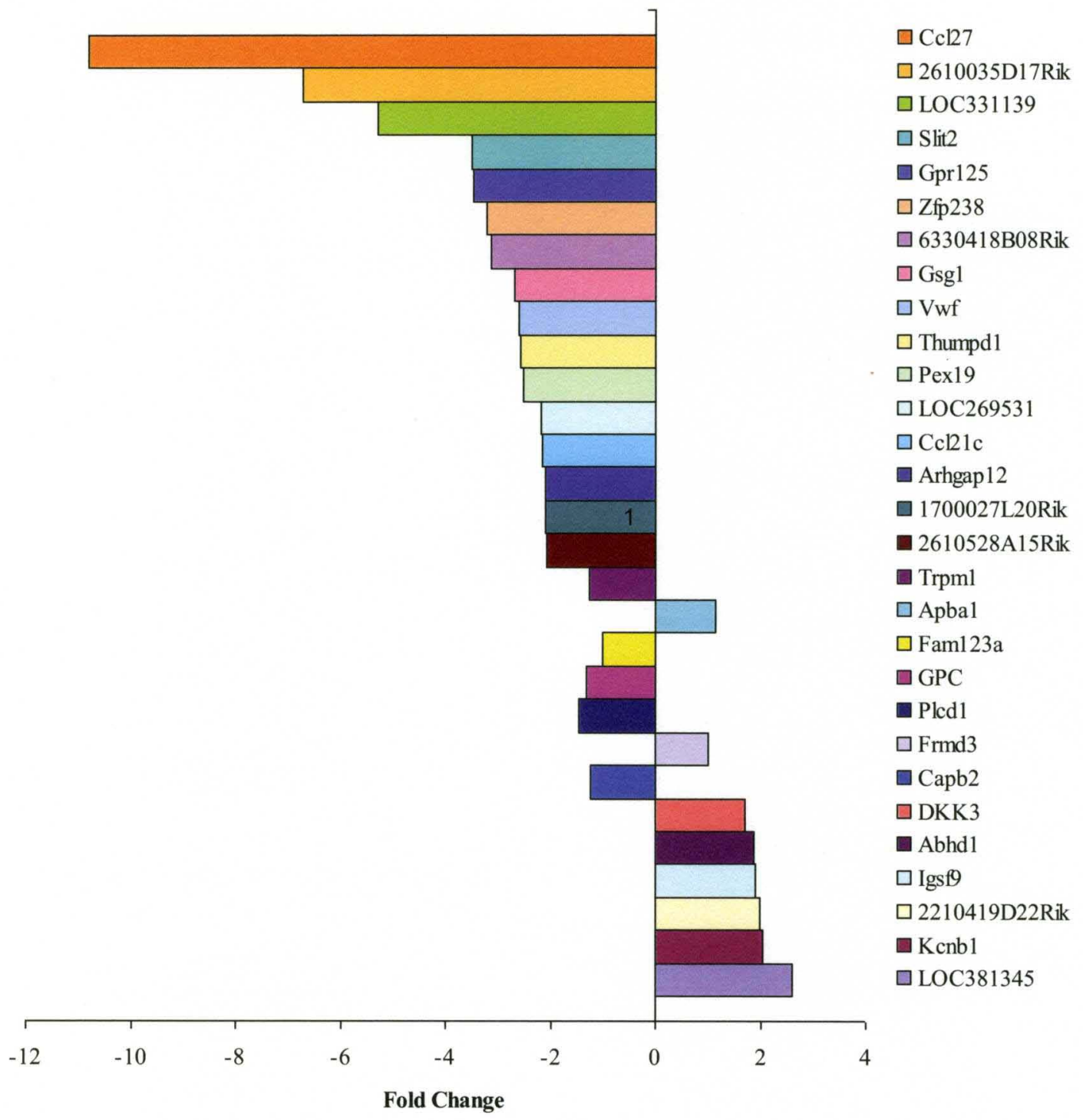


chosen but their relative quantity could not be determined because of the nature of their transcripts (Figure 32). Some of these transcripts (LOC381345, 2210419D22RIK, LOC296531, 6330418B08RIK, LOC331139) have no sequence annotated at Ensemble. Sequences of clones from RIKEN have multiple open reading frames in each transcript. For 2610035D17RIK, there are four different transcripts annotated at Ensembl but none code for a protein (Figure 31). Within each transcript there are different open reading frames. The genes selected, the p-value, the fold change and the reason they were selected is summarized in Table 2. The expression of the selected genes was determined and verified using qPCR.

\section{Genes differentially expressed in $N y x^{n o b}$ mice at P13}

A total of 24 genes were selected for confirmation of their differential expression between the $N y x^{n o b}$ and $C 57 B L / 6 J$ by qPCR (Figure 33). The relative expression level of these genes was determined using the $\Delta \Delta \mathrm{CT}$ method. From Figure 33, seven out of the twenty-five genes showed a significant change in expression as determined by t-test $(\mathrm{p}<0.05)$. Among the genes that were significantly down regulated are Trpml $(>55 \%)$, Apbal (>35\%), VWF (>55\%), Slit2 (>55\%), DKK3 (>35\%), Fam123a (>45\%), and GPC $(>45 \%)$ (Figure 33). DKK3 showed a 1.7 fold (Figure 32) increase in the array analysis but the qPCR result showed a $35 \%$ decrease. The majority of the genes show no change in expression in $N y x^{n o b}$ mice compared $C 57 B L / 6 J$. For example, in the array analysis, Ccl27 is down regulated about 10 fold; however in qPCR there is no significant difference in expression between the $N y x^{n o b}$ mice and C57BL/6J. IGSF9 and Abdhl both have trend toward an increase but this was not statistically different. More samples need to be analyzed to confirm this change (Figure 33 . For the rest of the genes (Cabp2, 
Cclc21, Frmd3, Thump1, GPhotoreceptor125, Abdh1, Gde, Kcnb1, ZFP238, Gsg1, 2610528A15Rik, 1700027L20Rik) no significant differences was obtained using t-test.

\section{Candidate Bipolar specific genes determined by their temporal expression}

\section{pattern}

The temporal expression pattern of retinal genes have been shown to provide insight into the cellular layers in which they are expressed (Kim et al., 2008). Genes expressed in bipolar cells have peak expression at P13 and decline in adulthood. Photoreceptor specific genes increase expression gradually and peak at adulthood. To further analyze which genes are most likely to be expressed in bipolar cells, I determined the temporal expression pattern of each gene differentially expressed in $N y x^{n o b}$ retina. Figure 34 shows the temporal expression pattern of these nine genes. As a positive control for bipolar cells specific genes, Grm6, and $N y x$ were analyzed. Rhodopsin and bassoon were used as controls for photoreceptor specific genes. Plcdl and Arhgap 12 were added because they had good p-value, fold change and were shown to be bipolar specific genes. The temporal expression profiles of genes in blue (Trpm1, VWF, Apbal, and $G P C$ ) follow a bipolar specific expression profile. These genes have minimal expression levels during the early part of retinal development (P1, P3 and P5), however their expression increases from P7 until it reaches a peak at P13 (Figure 34). In contrast, the expression level of photoreceptor specific genes increases steadily as the retina develops with maximal expression at adulthood. From the pool of genes that showed differential expression on qPCR only Fam123a follows the photoreceptor specific profile (Figure 34). Several other genes (Slit2, DKK3, Plcd1, Arhgap12) follow neither the 
Figure 33. qPCR analysis of genes that showed significantly different expression level between $N y x^{n o b}$ and $C 57 B L / 6 J$ as determined by t-test. Genes showing statistical difference between $C 57 B L / 6 J$ and $N y x^{n o b}$ are indicated by $*$ (i.e. $*<0.05, * *<0.005$, $* * *<0.001)$. Data represent mean $+\mathrm{SE}(\mathrm{n}=4)$. 


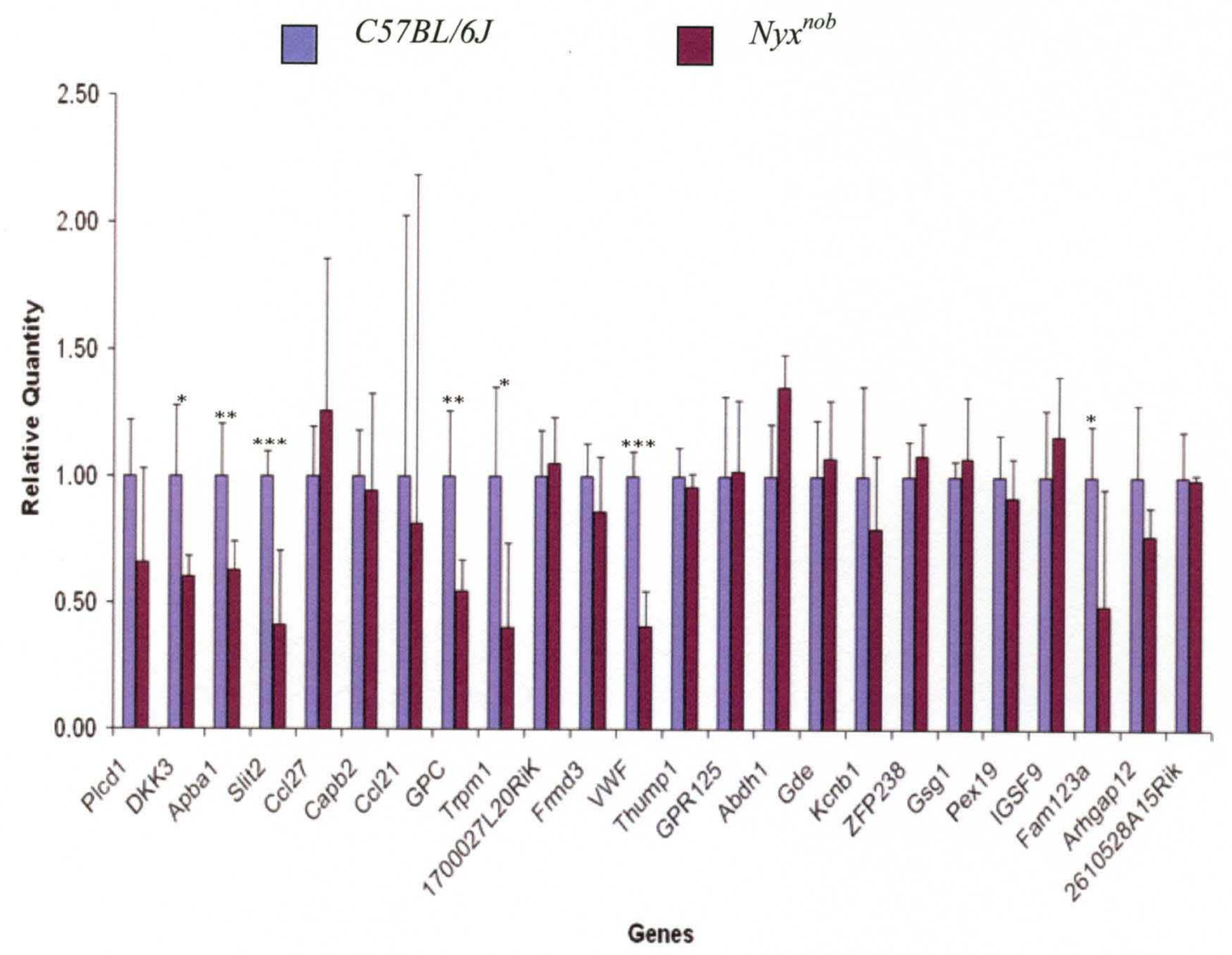


Figure 34. Temporal expression pattern of genes differentially regulated in $N y x^{n o b}$ mice. The data represent means of 3 replicates of retinas from C57B6L/6J mice. Genes that follow the expression profile of bipolar cells are in blue (VWF, Apbal, GPC). Grm6, Nyx and Trpml are controls for bipolar specific genes. Genes that follow the expression profile of photoreceptors are in brown (Fam123a). Rhodopsin (Rho) and bassoon are controls for photoreceptor specific genes. Genes that have neither Photoreceptor nor Bipolar cell expression profile are shown in magenta (Slit2, DKK3, Plcd1, Arhgap12). These genes are likely to be expressed in multiple cell types. 


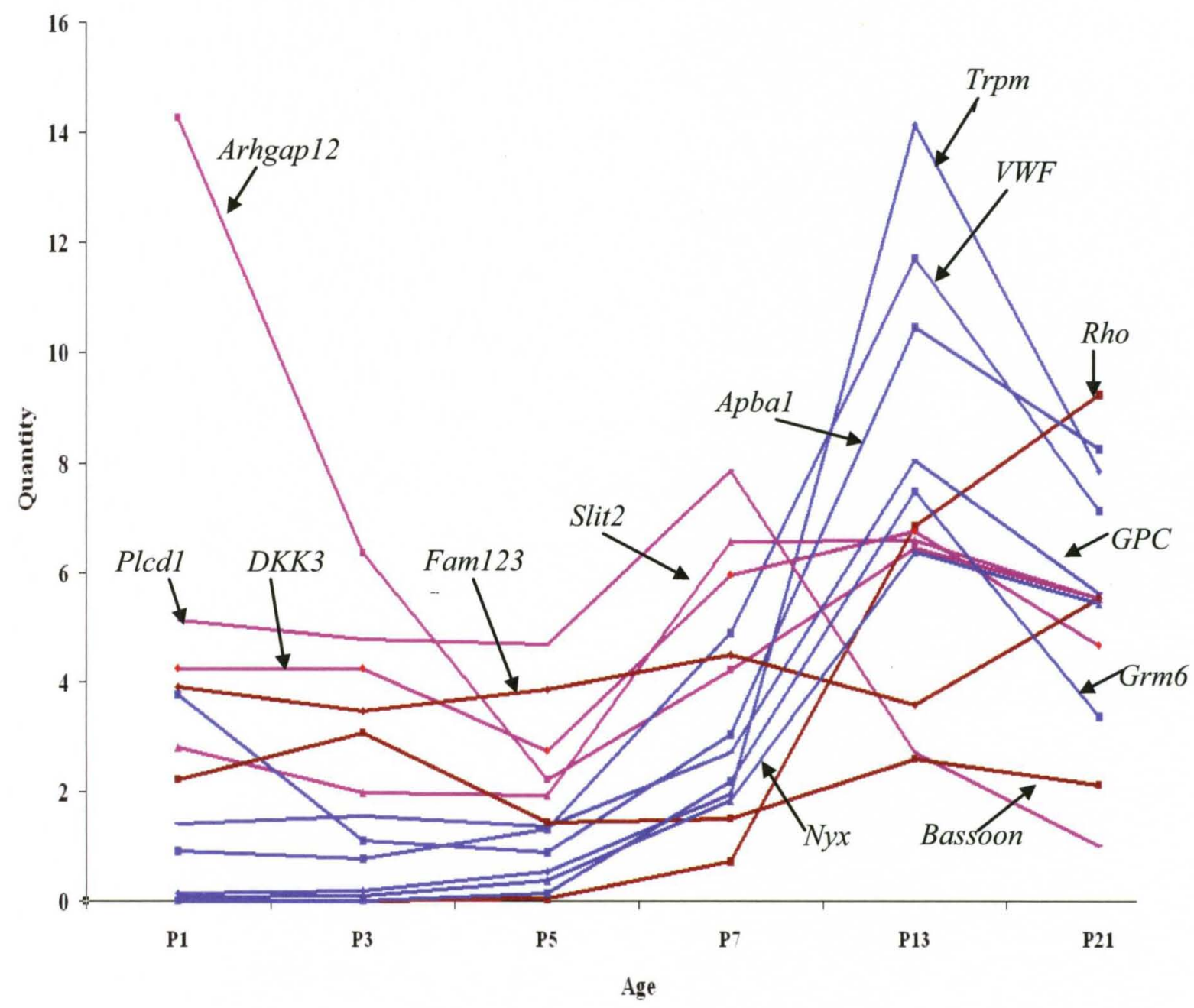


bipolar nor photoreceptor specific profile. These genes are shown in magenta. They have high expression level during the early part of retinogenesis with maximal expression at $\sim$ P7. These genes might be expressed in two or more different types of cells in the retina.

\section{DISCUSSION}

The experiments in this chapter suggest that abnormal waves in $N y x^{n o b}$ retina might affect the expression of several genes. The expression of voltage dependent calcium channels (VDCC) was decreased for most of the time points during development. The reduction in the mRNA level of these channels might be to mitigate the effect of increase glutamate released on to the OPL. Since the Grm6 signaling cascade at the tips of the depolarizing bipolar cell is defective, the cells might be reducing the levels of voltage dependent calcium channel to reduce the amount of glutamate release into the synaptic cleft (OPL). Cacnalf and Cacnb2 encode the $\alpha$-subunit and $\beta$ subunit of voltage dependent calcium channel. Calcium entry through these channels leads to the release of glutamate into the OPL. Also the level of Cacnalf mRNA in $N y x^{n o b}$ and Cacnalf $f^{n o b 2}$ are almost the same at most of the time points. However the Cacnalf $f^{\text {ob2 }}$ mouse is not a real knockout (Doering et al., 2008). It is reported that an in frame stop codon in exon 2 is spliced out in $10 \%$ of the mRNAs in this mouse. The truncated Cacnalf gene generates a functional protein that can be detected in western blots (Doering et al., 2008). So the VDCC in $N y x^{n o b}$ might be functioning at levels similar to those in Cacnalf ${ }^{\text {nob2 }}$.

The mRNA level of Grm6 increase about four fold at P13 in $N y x^{n o b}$ retinas. This is the time of active synaptogenesis. In general, the mRNA levels of all synaptic genes increase at this time point. The increase in the mRNA levels of Grm6 might be playing a 
compensatory role. In the initial period of synaptogenesis, Grm6 mRNA level might increase substantially because of defective signaling in the depolarizing bipolar cell. Glutamate release into the OPL activates the Grm6 receptors at the tips of depolarizing bipolar cell which in turn activate a G-coupled signal transduction cascade that closes the Trpml channel. So the transient increase in the mRNA level of Grm6 at P13 might be to compensate for the defective signaling in depolarizing bipolar cells at the time of synapse maturation.

In contrast, the Cacnalf ${ }^{\text {nob2 }}$ retina shows a delay of 8 days in peak expression of all genes. Instead of expression peaking at P13, all OPL genes analyzed have peak expression level between P21 to P26. This shows another difference between CSNB1 and CSNB2. In the $N y x^{n o b}$ retina despite changes that I have mentioned, the temporal expression pattern of all OPL genes are similar to $C 57 B L / 6 J$.

Microarray data show that the fold changes are dependent on the probe sets used. This can be explained by the fact that genes consist of several isoforms and splice variants. If a probe set is measuring the mRNA level of one isoform or splice variant, the fold change will be different from when it is measuring the levels for two or more forms of the same gene. These limit the confidence that one has in the change in expression of a particular gene. Of the twenty five genes I examined, only seven show significant differences in expression in $N y x^{n o b}$ retinas compare to $C 57 B L / 6 J$. It might be that the qPCR primers I designed are not measuring rares transcript that the array probe measures. In addition, some of the genes that show significant difference in expression between the $N y x^{n o b}$ and $\mathrm{C} 57 \mathrm{~B} / 6 \mathrm{~J}$ retinas in $\mathrm{qPCR}$ did not show any changes in microarray data. The mRNA levels of both Trpml and Apbal were analyzed because they interacted with 
nyctalopin in the yeast two hybrid screens and both of these genes showed a significant difference in expression in qPCR. These data indicate that more array replicates are needed, verification is essential and important genes with real change may have been missed.

To determine if the genes that show differential expression are expressed in bipolar cells. I examined their temporal expression profile. These data again show that some of the genes that change expression levels in the $N y x^{n o b}$ retina do not show a bipolar specific profile. Nyctalopin has been shown to be expressed in the IPL of the retina (Morgans et al., 2006). Synapses at the IPL appeared before synapses at the OPL (Sidman, 1961; Young, 1985; Marquardt and Gruss, 2002). Perhaps some of these genes might be localized to the IPL and be functionally tied to nyctalopin in IPL. This might explain the temporal expression profile of those genes that do not exactly follow bipolar specific profile. However, it is been shown that specific expression of nyctalopin at is tips of depolarizing bipolar cell is sufficient to rescue all $N y x^{n o b}$ phenotypes studied (Demas et al., 2006; Gregg et al., 2007). It might also be that these genes might be expressed in some other neurons in the retina most likely photoreceptors.

Overall, three key findings were obtained from these experiments. The reduced mRNA levels of Trpml, VWF and Apbal. These findings indirectly show a connection between these proteins and loss of nyctalopin. In addition, the temporal expression profiles of these two genes ( $V W F$ and $A p b a l$ ) follow bipolar specific genes. Trpm1 is the non-selective cation channel in depolarizing bipolar cell (Shen et al., 2009; Morgan et al., 2009; Koike et al., 2009). The proper function of this channel in depolarizing bipolar cell has been previously tied to nyctalopin (Gregg et al., 2007). In addition, I have determined 
that nyctalopin directly interacted with Trpm1 (Chapter III). These data provide the first interacting partner of nyctalopin. Apbal is an adapter protein that is involved in vesicular transport in neurons. It has been shown to directly interact with kinesin 17, which is part of a molecular motor (Guillaud et al., 2007). Further work need to be done to confirm the role of Apbal in depolarizing bipolar cell. ERG could be performed on the Apba1 knockout mouse. This would show if the b-wave is present in this mice. Also immunohistochemical analysis could be done on C57BL/6J, $N y x^{n o b}$, and $\operatorname{Trpml}^{-1-}$ to determine the localization of Apbal. VWF has been shown to interact with decorin, which is in the SLRP family as nyctalopin (Guidetti et al., 2004). The VWF domain has been involved in the trafficking of voltage dependent calcium channels to the plasma membrane (Canti et al., 2005). As a scaffold, a retina specific VWF could be involved in nyctalopin mediated trafficking of Trpml channel to the plasma membrane.

Although preliminary, these analyses have provided some genes that could be part of the Grm6 signaling cascade to the Trpml channel in depolarizing bipolar cell. 


\section{APPENDIX II}

\section{LIST OF ABBREVIATIONS}

AMPA

Ada

DAG

CMM

CSNB

$\mathrm{Cub}$

$\mathrm{Ca}^{2+}$

ERG

Grm6

$\mathrm{G} \alpha$

$\mathrm{G} \beta$

$\mathrm{G} \gamma$

GPI

His

IPL

IP3

iGluR

Kir2.1 $\alpha$-amin-3-hydroxy-5-methyl-4 isoxazolepropionate

Adenine

Diacylglycerol

Canine microsomal membrane

Congenital stationary night blindness

C-terminus of ubiquitin

Calcium

Electroretinogram

metabotropic glutamate receptors 6

Alpha ( $\alpha$ ) subunit of G-protein

Beta $(\beta)$ subunit of G-protein

Gamma $(\gamma)$ subunit of G-protein

Glycosylphosphatidylinositol

Histidine

Inner plexiform layer

Inositol Trisphosphate

Ionotropic glutamate receptors

Potassium channel isoform 2.1 


\begin{tabular}{|c|c|}
\hline LRR & Leucine rich repeat \\
\hline Leu & Leucine \\
\hline LP & Loop \\
\hline Luc & Luciferase gene \\
\hline L-APB & L-2-1 amino-4-phosphonobutyrate \\
\hline mGluR & metabotropic glutamate receptors \\
\hline NubI & N-terminus of ubiquitin \\
\hline NubG & $\begin{array}{l}\text { Isoleucine }(\mathrm{I}) \text { to glycine }(\mathrm{G}) \text { substitution at } \mathrm{N} \text {-terminus of } \\
\text { ubiquitin }\end{array}$ \\
\hline$N y x^{n o b}$ & No b-wave mice \\
\hline Nyc & Nyctalopin \\
\hline OPL & Outer plexiform layer \\
\hline PIP2 & Phosphoinositol bisphosphate \\
\hline PDE & Phosphodiesterase \\
\hline RGC & Retinal ganglion cells \\
\hline RGS & Regulator of G-protein signaling \\
\hline Rho & Rhodopsin \\
\hline RPE & Retinal pigment epithelium \\
\hline ROS & Photoreceptor outer segment \\
\hline RNC & Ribosome nascent chain \\
\hline SLRP & Small leucine rich proteoglycan \\
\hline $\mathrm{SD}$ & Synthetic dropout media \\
\hline SUC & S. cerevisiae invertase signal sequence \\
\hline
\end{tabular}


SS/S

SRP

$\operatorname{Trp}$

Trpm 1

Trpv1

qPCR

VDCC
Signal sequence

Signal recognition particle

Tryptophan

Transient receptor channel melastatin 1

Transient receptor channel villinoid 1

Quantitative polymerase chain reaction

Voltage dependent calcium channel 


\section{CURRICULUM VITAE}

Name

Phone

E-mail

Education

2007-2010

$2004-2007$

2000-2004

Other Experience

1998-1999

\section{Leadership}

2002- 2004

2004-summer

$2005-2006$
Pasano Bojang Jr

5024358300

ppenku@hotmail.com

P0boja01_alouisville.edu

Ph.D. Department of Biochemistry and Molecular Biology University of Louisville School of Medicine Louisville KY, U.S.A.

MA. Department of Biochemistry and Molecular Biology University of Louisville School of Medicine Louisville, KY, U.S.A.

B.Sc. Department of Chemistry, Kentucky State University, Frankfort, KY, USA.

British medical research council (MRC), The Gambia, West Africa. Malaria Program: Laboratory Assistant Supervisor: Dr. Lorenz Von seidlein.

Peer to peer mentorship

Kentucky State University, Frankfort, KY, U.S.A.

Teaches SAT and ACT preparation courses

Kentucky state University, Frankfort, KY.

Vice president of the black biomedical graduate student Organization -University of Louisville, Louisville, KY. 
Awarded WHO/ Gambia government scholarship to study medicine at the University of the Gambia (UTG) Left for the U.S.A before UTG officially opened.

UTG is Gambia's first and only university (1999)

President's Award

Kentucky State University, Frankfort, KY, U.S.A (2001)

United States National Dean List (2001),

United States All American Scholar (2001-2003) and

United States Achievement Academy honoree (2001).

Certificate of Merit- Whitney Young College,

Kentucky State University, Frankfort, KY, U.S.A (2002)

Certificate of recognition and achievement, Whitney Young College, Kentucky State University, Frankfort, KY, U.S.A (2002)

Academic Achievement in chemistry, chemistry department, Kentucky State University, Frankfort, KY, U.S.A (2004)

Joseph $\mathrm{M}$ and Eula $\mathrm{C}$ Lawrence Travel scholarship to attend the Association of Research in Vision and Ophthalmology (ARVO) +annual meeting (2008).

\section{Paper Presentation}

Bojang P and Burkey T (2003) Synthesis of Arene Chromium complexes for photochromic materials. Amacrine cells-Abstract 931

Bojang P, Pearing J, Peachey N, Kamerman M, Gregg RG (2009) Nyctalopin interact with the transient receptor channel in yeast. ARVO- Abstract 5176.

\section{Posters}

Bojang P, Pearing J, Gregg RG (2008) Development expression of voltage dependent calcium channels (VDCCs) is altered in mice with congenital stationary night blindness $1 \& 2(C S N B 1 \& 2)$.ARVO-Abstract 1284

Pearring JN, Bojang P, Gregg RG (2009) Nyctalopin expression in Grm6 mutant mice and membrane topology. determine in yeast. ARVO-Abstract 1021 
Bojang P, and Gregg RG (2009) Nyctalopin interact with Trpml, a non specific cation channel required for depolarizing bipolar cells. BMB retreat (Honorary Mentioned)

Bojang $\mathbf{P}$ and Antonious GF (2003) Insecticides from Wild Tomato: Trichome Counts and Contents. Posters at the Capital- Abstract 52

\section{Publications}

Withers JR, Ruschman C, Bojang P, Perkin P, Holmes LM (2005) Synthesis and characterization of bi and Trimetallic octacyonometalate (IV) complexes. J. Inorg. Chem: 44(2):352-8.

Fox CW, David MG, Bojang P (2006) Genetic and environmental sources of variation in survival on nonnative host species in the generalist seed beetle, stator limbatue. Southwest Naturalist 51(4): 490-501.

Ngome ML, Bojang P, Falkner CK, Conklin DJ, Prough $\mathrm{RA}(2010)$ Murine hepatic aldehyde dehydrogenase lal is a major contributor to oxidation of aldehydes formed by lipid peroxidation. Chemical Biological Interactions (Submitted 8-01-10)

Bojang P, Pearing JN, Shen Y, Nawy S, Gregg RG (In Preparation) Nyctalopin is required to localize the TrpmI channel to the dendrites of the ON bipolar cells (First Coauthors Pasano Bojang and Jillian Pearring)

Bojang P, Pearring JN, Gregg RG (In Preparation for submission to JBC) Topological analysis of a small leucine-rich repeat Proteoglycan (SLRP), nyctalopin, in yeast and in vitro system. 